\title{
Asymmetric domino reaction of $\alpha, \beta$-unsaturated aldehydes and $\alpha$-acyl $\alpha, \beta$-unsaturated cyclic ketones catalyzed by diphenylprolinol silyl ether
}

\author{
Yujiro Hayashi*, Yurina Suga, Nariyoshi Umekubo \\ Department of Chemistry, Graduate School of Scince, Tohoku University, 6-3 \\ Aramaki Aza-Aoba, Aoba-ku, Sendai, Miyagi 980-8578, Japan
}

\section{Table of Contents}

1. Materials and Methods Page S2

2. Experimental Procedures. Page S3-S28

2.1 Preparation of 2-acetylcyclohex-2-en-1-one Page S3

2.2 Typical procedure of the vinylogous Michael reaction. .Page S3-S4

2.3 Compound information. Page S4-S9

2.4 Procedure of the silane reduction. Page S9

2.5 Wittig reaction Page S10

2.6 Preparation of cyclic $\alpha$-acyl- $\alpha, \beta$-unsaturated ketone Page S11-S14

2.7 Typical procedure of the vinylogous Michael reaction/Wittig reaction. Page S15

2.8 Compound information. .Page S15-S23

2.9 Procedure of $1 \mathrm{mmol}$ scale.

Page S24

2.10 Oxidation of compound $7 \mathbf{l}$. Page S24-S25

2.11 The determination of the absolute configuration of 9 Page S25-S28

3. Spectra for compounds Page S29-S182

4. Reference

.Page S183 


\section{Materials and Methods}

\section{General Methods.}

General Remark: All vinylogous Michael reactions were carried out under atmosphere and monitored by NMR and thin-layer chromatography using Merck 60 F254 precoated silica gel plates $(0.25 \mathrm{~mm}$ thickness). Specific optical rotations were measured using a JASCO P-1020 polarimeter and a JASCO DIP-370 polarimeter. FT-IR spectra were recorded on a JASCO FT/IR-410 spectrometer and a Perkin Elmer spectrum BX FT-IP spectrometer. ${ }^{1} \mathrm{H}$ and ${ }^{13} \mathrm{C}$ NMR spectra were recorded on an Agilent-400 MR (400 MHz for ${ }^{1} \mathrm{H} \mathrm{NMR}, 100 \mathrm{MHz}$ for ${ }^{13} \mathrm{C} \mathrm{NMR}$ ) instrument. Data for ${ }^{1} \mathrm{H}$ NMR are reported as chemical shift $(\delta \mathrm{ppm})$, integration multiplicity $(\mathrm{s}=$ singlet, $\mathrm{d}=$ doublet, $\mathrm{t}=$ triplet, $\mathrm{q}=$ quartet, $\mathrm{dd}=$ doublet of doublets, $\mathrm{ddd}=$ doublet of doublet of doublets, $\mathrm{dt}=$ double of triplets, $\mathrm{m}=$ multiplet), coupling constant (Hz), Data for ${ }^{13} \mathrm{C}$ NMR are reported as chemical shift. High resolution ESI-TOF mass spectra were measured by Themo Orbi-trap instrument. HPLC analysis was performed on a HITACHI Elite LaChrom Series HPLC, UV detection monitored at appropriate wavelength respectively, using CHIRALPACK ${ }^{\circledR}$ IA $(0.46 \mathrm{~cm} \times 25 \mathrm{~cm})$, CHIRALPACK $^{\circledR}$ IB $(0.46$ $\mathrm{cm} \times 25 \mathrm{~cm})$, CHIRALPACK ${ }^{\circledR}$ IC $(0.46 \mathrm{~cm} \times 25 \mathrm{~cm})$ and CHIRALPACK ${ }^{\circledR} \operatorname{IF}(0.46 \mathrm{~cm} \times 25 \mathrm{~cm})$. Meltingpoint apparatus was Yanaco MP-J3. All the reagents were purchased from commercial sources (ALDRICH, Combi-Blocks, FUJIFILM Wako Chemicals, KANTO CHEMICAL, TCI), used without further purification. 


\section{Experimental Procedures}

\subsection{Preparation of 2-acetylcyclohex-2-en-1-one ${ }^{1}$}

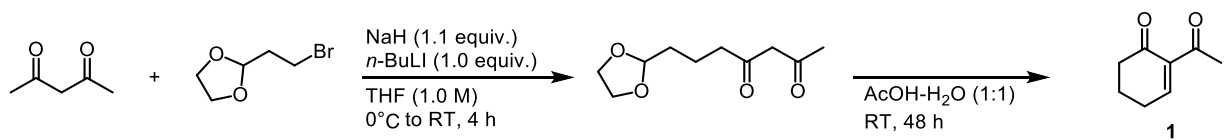

To a suspension of $\mathrm{NaH}(60 \mathrm{wt} \%, 440 \mathrm{mg}, 11 \mathrm{mmol})$ in THF $(9.0 \mathrm{~mL})$, acetylacetone $(1.0 \mathrm{~mL}, 10 \mathrm{mmol}) \mathrm{was}$ added dropwise at $0{ }^{\circ} \mathrm{C}$ under $\mathrm{N}_{2}$ atmosphere within $20 \mathrm{~min}$. After $10 \mathrm{~min}, n$-BuLi (1.4 M in $n$-Hexane, $7.0 \mathrm{~mL}, 10$ mmol) was added dropwise within $10 \mathrm{~min}$ for $15 \mathrm{~min}$ at $0{ }^{\circ} \mathrm{C}$. Then 2-(2-Bromoethyl)-1,3-dioxolane $(1.2 \mathrm{~mL}, 10$ mmol) was added. The mixture was stirred for $4 \mathrm{~h}$ at room temperature. The reaction was quenched by the addition of sat. aq. $\mathrm{NH}_{4} \mathrm{Cl}(10 \mathrm{~mL})$, diluted with $\mathrm{H}_{2} \mathrm{O}(10 \mathrm{~mL})$. Separated aqueous layer was extracted with diethyl ether $(30$

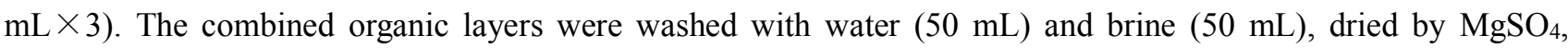
filtered and concentrated in vacuo. The residue was purified by flash chromatography on silica gel ( $n$-Hexane: EtOAc $=3: 1)$ to give compound diketone as a yellow oil.

A solution of diketone in $\mathrm{AcOH}-\mathrm{H}_{2} \mathrm{O}$ mixture $(1: 1,50 \mathrm{~mL})$ was stirred at room temperature. After 2 days, the mixture was extracted with diethyl ether $(50 \mathrm{~mL} \times 4)$. The combined organic layers were neutralized with sat. aq. $\mathrm{NaHCO}_{3}(200 \mathrm{~mL})$, washed with $\mathrm{H}_{2} \mathrm{O}(200 \mathrm{~mL})$ and brine $(200 \mathrm{~mL})$ dried by $\mathrm{MgSO}_{4}$. filtered and concentrated in vacuo. The residue was purified by flash chromatography on silica gel $(n$-Hexane: EtOAc $=4: 1)$ to give compound 1 (510 mg, 37\% in two steps) as a yellow liquid.

\section{2-Acetylcyclohex-2-en-1-one (1)}<smiles>CC(=O)C1=CCCCC1=O</smiles>

Keto: Enol $=2: 1$

Yield: $37 \%$ (510 mg, two steps)

Physical State: yellow liquid

${ }^{1}$ H NMR (400 MHz, CDCl $) \delta 7.67(\mathrm{t}, J=4.0 \mathrm{~Hz}, 1 \mathrm{H}), 6.23(\mathrm{dt}, J=10.0,1.2 \mathrm{~Hz}, 0.5 \mathrm{H}), 5.59(\mathrm{dt}, J=9.2,4.0 \mathrm{~Hz}$, $0.5 \mathrm{H}), 2.56-2.51(\mathrm{~m}, 4 \mathrm{H}), 2.46(\mathrm{~s}, 3 \mathrm{H}), 2.39-2.34(\mathrm{~m}, 1 \mathrm{H}), 2.11(\mathrm{~s}, 1.5 \mathrm{H}), 2.08-2.01(\mathrm{~m}, 2 \mathrm{H})$

\subsection{Typical procedure of the vinylogous Michael reaction}

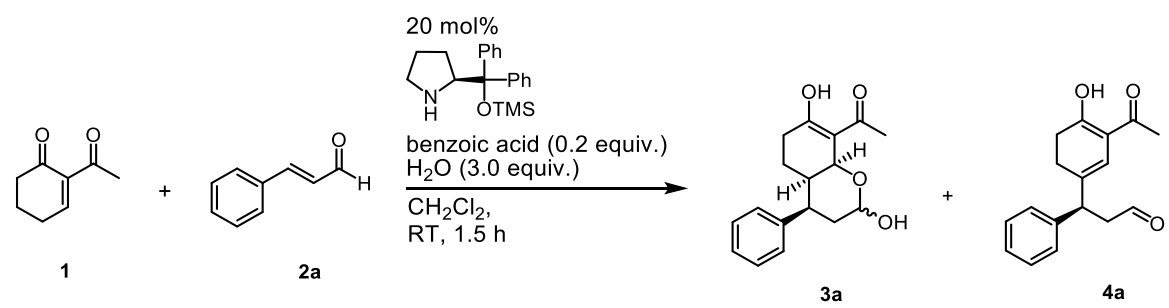

To a solution of ketone 1 (40 mg, $0.30 \mathrm{mmol})$ and aldehyde $\mathbf{2 a}(63 \mathrm{mg}, 0.45 \mathrm{mmol})$ in $\mathrm{CH}_{2} \mathrm{Cl}_{2}(300 \mu \mathrm{L}), \mathrm{H}_{2} \mathrm{O}(16$ $\mu \mathrm{L}, 0.90 \mathrm{mmol})$, benzoic acid $(7.0 \mathrm{mg}, 0.060 \mathrm{mmol})$, and catalyst $(20 \mathrm{mg}, 0.060 \mathrm{mmol})$ were added at room temperature. After confirming the disappearance of aldehyde by TLC or ${ }^{1} \mathrm{H}-\mathrm{NMR}$, the reaction was quenched by 
the addition of sat. aq. $\mathrm{NaHCO}_{3}(3 \mathrm{~mL})$. Separated aqueous layer was extracted with $\mathrm{CH}_{2} \mathrm{Cl}_{2}(5 \mathrm{~mL} \times 3)$. The combined organic layers were washed with brine $(10 \mathrm{~mL})$, dried by $\mathrm{Na}_{2} \mathrm{SO}_{4}$, filtered and concentrated in vacuo. The residue was purified by flash chromatography on silica gel $(n$-Hexane: EtOAc $=10: 1 \sim 3: 1)$ to give compound 3a (54 mg, 67\%, dr = 1:1) and $4 \mathbf{a}(10 \mathrm{mg}, 13 \%)$.

\subsection{Compounds information}

\section{1-((4R,4aR,8aS)-2,7-Dihydroxy-4-phenyl-3,4,4a,5,6,8a-hexahydro-2H-chromen-8-yl)ethan-1-one (3a)}

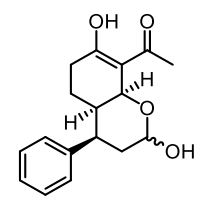

$\mathrm{dr}=1: 1$

Yield: $67 \%(54.3 \mathrm{mg})$

Physical State: pink amorphous

${ }^{1}$ H NMR (400 MHz, $\left.\mathbf{C D C l}_{3}\right)$ 8 7.37-7.33 (m, 4H), 7.28-7.23 (m, 3H), 7.20-7.17 (m, 3H), 5.51 (d, $\left.J=3.2 \mathrm{~Hz}, 1 \mathrm{H}\right)$, $5.15(\mathrm{~s}, 1 \mathrm{H}), 5.01(\mathrm{dd}, J=8.8,2.4 \mathrm{~Hz}, 1 \mathrm{H}), 4.54(\mathrm{~s}, 1 \mathrm{H}), 3.69(\mathrm{dt}, J=14.0,4.0 \mathrm{~Hz}, 1 \mathrm{H}), 3.27(\mathrm{dt}, J=13.2, .4 .0 \mathrm{~Hz}$, $1 \mathrm{H}), 2.40-2.38(\mathrm{~m}, 1 \mathrm{H}), 2.36-2.34(\mathrm{~m}, 1 \mathrm{H}), 2.33(\mathrm{~s}, 3 \mathrm{H}), 2.32(\mathrm{~s}, 3 \mathrm{H}), 2.24(\mathrm{dd}, J=13.2,4.0 \mathrm{~Hz}, 1 \mathrm{H}), 2.21-2.11(\mathrm{~m}$, 2H), 2.01-1.93 (m, 2H), 1.89-1.79 (m, 4H), 1.19-1.12 (m, 2H)

${ }^{13} \mathbf{C}$ NMR (100 MHz, $\left.\mathbf{C D C l}_{3}\right) \delta$ 200.5, 200.5, 185.6, 185.3, 142.3, 141.5, 128.5, 128.5, 128.4, 128.4, 127.4, 127.4, 127.3,127.3, 126.6, 126.4, 108.9, 108.6, 96.4, 91.9, 72.2, 65.2, 42.8, 39.9, 39.3, 37.1, 32.0, 31.2, 31.1, 28.9, 23.9, $23.9,15.4,14.6$

IR (neat) $v 3420,2949,2883,1606,1496,1416,1363,1310,1254,1209,1134,1056,1008,956,895,956,895$, 757 ,

$702 \mathrm{~cm}^{-1}$

HRMS (ESI) m/z: $[\mathrm{M}+\mathrm{H}]^{+}$Calcd for $\mathrm{C}_{17} \mathrm{H}_{20} \mathrm{O}_{4} \mathrm{H}^{+}$289.1435; Found 289.1435

$[\alpha]_{\mathrm{D}}^{26}+31.1\left(c 1.42, \mathrm{CHCl}_{3}\right)$

$\mathbf{R}_{\mathbf{f}}(n$-Hexane: EtOAc $=3: 1$, color reagent: $p$-anisaldehyde $): 0.10$

(R)-3-(3-Acetyl-4-hydroxycyclohexa-1,3-dien-1-yl)-3-phenylpropanal (4a)<smiles>CC(=O)c1c(O)cccc1CC=O</smiles>

Yield: $13 \%(10.4 \mathrm{mg})$

Physical State: yellow oil

${ }^{1} \mathbf{H}$ NMR (400 MHz, CDCl $) \delta 9.74(\mathrm{dd}, J=2.8,1.6 \mathrm{~Hz}, 1 \mathrm{H}), 7.36-7.34(\mathrm{~m}, 2 \mathrm{H}), 7.28-7.24(\mathrm{~m}, 3 \mathrm{H}), 6.13(\mathrm{~d}, J=$ $1.2 \mathrm{~Hz}, 1 \mathrm{H}), 4.02$ (t, $J=7.6 \mathrm{~Hz}, 1 \mathrm{H}), 2.98$ (ddd, $J=16.4,8.0,2.4 \mathrm{~Hz}, 1 \mathrm{H}), 2.89$ (ddd, $J=16.4,7.2,1.6 \mathrm{~Hz}, 1 \mathrm{H}$ ), 
$2.41(\mathrm{td}, J=7.6,4.0 \mathrm{~Hz}, 2 \mathrm{H}), 2.28-2.14(\mathrm{~m}, 2 \mathrm{H}), 2.16(\mathrm{~s}, 3 \mathrm{H})$

${ }^{13}$ C NMR (100 MHz, CDCl $) \delta$ 201.2, 199.2, 180.0, 141.4, 132.7, 128.7, 128.7, 127.6, 127.6, 127.0, 118.0, 107.9, $47.1,45.9,35.0,24.9,19.6$

IR (neat) $v 3431,2954,2936,1721,1603,1494,1411,1268,1218,1030,954,760,703 \mathrm{~cm}^{-1}$

HRMS (ESI) m/z: [M+H] ${ }^{+}$Calcd for $\mathrm{C}_{17} \mathrm{H}_{18} \mathrm{O}_{3} \mathrm{H}^{+}$271.1329; Found 271.1332

$[\alpha]_{\mathrm{D}}^{25}+45.9\left(c 1.04, \mathrm{CHCl}_{3}\right)$

$\mathbf{R}_{\mathbf{f}}(n$-Hexane: EtOAc $=3: 1$, color reagent: $p$-anisaldehyde): 0.35

1-((4R,4aR,8aS)-4-(4-Bromophenyl)-2,7-dihydroxy-3,4,4a,5,6,8a-hexahydro-2H-chromen-8-yl)ethan-1-one (3b)

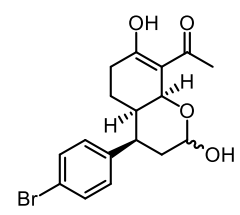

$\mathrm{dr}=1: 1$

Yield: 70\% (77.4 mg)

Physical State: pink amorphous

${ }^{1}$ H NMR (400 MHz, CDCl $)$ ) $7.48-7.45(\mathrm{~m}, 4 \mathrm{H}), 7.06-7.03(\mathrm{~m}, 4 \mathrm{H}), 5.51(\mathrm{~d}, J=2.8 \mathrm{~Hz}, 1 \mathrm{H}), 5.12(\mathrm{~s}, 1 \mathrm{H}), 4.99$ (d, $J=9.2 \mathrm{~Hz}, 1 \mathrm{H}), 4.51(\mathrm{~s}, 1 \mathrm{H}), 3.64(\mathrm{~d}, J=13.6 \mathrm{~Hz}, 1 \mathrm{H}), 3.22$ (d, $J=13.2 \mathrm{~Hz}, 1 \mathrm{H}) 2.40-2.34(\mathrm{~m}, 2 \mathrm{H}), 2.31-2.30$ (m, 6H), 2.20-2.12 (m, 3H), 1.98-1.95 (m, 1H), 1.92-1.87 (m, 1H), 1.83-1.79 (m, 4H), 1.29-1.23 (m, 1H), 1.13-1.09 $(\mathrm{m}, 2 \mathrm{H})$

${ }^{13}$ C NMR (100 MHz, CDCl $) \delta$ 200.5, 200.4, 185.4, 141.4, 140.5, 131.8, 131.6, 131.5, 129.3, 129.1, 129.0, 120.5, $120.3,108.8,96.2,91.9,72.2,65.2,47.0,45.2,42.4,39.8,39.2,36.8,35.0,31.9,31.1,31.0,28.7,24.8,24.0,23.9$, $15.4,14.6$

IR (neat) $v 2944,2884,1607,1490,1414,1207,1010,736,518,445 \mathrm{~cm}^{-1}$

HRMS (ESI) m/z: $[\mathrm{M}+\mathrm{Na}]^{+}$Calcd for $\mathrm{C}_{17} \mathrm{H}_{19}{ }^{79} \mathrm{BrO}_{4} \mathrm{Na}^{+} 389.0359$; Found 389.0359

HRMS (ESI) m/z: $[\mathrm{M}+\mathrm{Na}]^{+} \mathrm{Calcd}$ for $\mathrm{C}_{17} \mathrm{H}_{19}{ }^{81} \mathrm{BrO}_{4} \mathrm{Na}^{+}$391.0340; Found 391.0340

$[\alpha]_{\mathrm{D}}^{29}+24.2\left(c 0.780, \mathrm{CHCl}_{3}\right)$

$\mathbf{R}_{\mathbf{f}}(n$-Hexane: EtOAc $=3: 1$, color reagent: $p$-anisaldehyde): 0.10

(R)-3-(3-Acetyl-4-hydroxycyclohexa-1,3-dien-1-yl)-3-(4-bromophenyl)propanal (4b)<smiles>CC(=O)c1ccc(C(CC=O)c2ccc(Br)cc2)cc1</smiles>

Yield: $25 \%(26.3 \mathrm{mg})$

Physical State: yellow oil

${ }^{1}$ H NMR (400 MHz, CDCl $) \delta 9.72(\mathrm{~s}, 1 \mathrm{H}), 7.44$ (d, $\left.J=8.4 \mathrm{~Hz}, 2 \mathrm{H}\right), 7.11(\mathrm{~d}, J=8.4 \mathrm{~Hz}, 2 \mathrm{H}), 6.11$ (s, 1H), 3.97 (t, 
$J=7.6 \mathrm{~Hz}, 1 \mathrm{H}), 2.98-2.83(\mathrm{~m}, 2 \mathrm{H}), 2.54-2.51(\mathrm{~m}, 1 \mathrm{H}), 2.43-2.38(\mathrm{~m}, 2 \mathrm{H}), 2.15-2.11(\mathrm{~m}, 1 \mathrm{H}), 2.14(\mathrm{~s}, 3 \mathrm{H})$

${ }^{13}$ C NMR (100 MHz, CDCl $) \delta$ 200.5, 199.1, 179.3, 140.6, 132.1, 131.9, 131.9, 129.4, 129.4, 120.9, 118.4, 107.9, $47.1,45.2,35.0,24.9,19.7$

IR (neat) $v 2932,1720,1606,1489,1407,1362,1269,1213,1074,1010,735 \mathrm{~cm}^{-1}$

HRMS (ESI) m/z: $[\mathrm{M}+\mathrm{Na}]^{+}$Calcd for $\mathrm{C}_{17} \mathrm{H}_{18}{ }^{79} \mathrm{BrO}_{3} \mathrm{Na}^{+}$349.0436; Found 349.0434

HRMS (ESI) m/z: $[\mathrm{M}+\mathrm{Na}]^{+} \mathrm{Calcd}$ for $\mathrm{C}_{17} \mathrm{H}_{18}{ }^{81} \mathrm{BrO}_{3} \mathrm{Na}^{+}$351.0414; Found 351.0417

$[\alpha]_{\mathrm{D}}^{27}+30.3\left(c 0.590, \mathrm{CHCl}_{3}\right)$

$\mathbf{R}_{\mathbf{f}}(n$-Hexane: EtOAc $=3: 1$, color reagent: $p$-anisaldehyde) 0.35

1-((4R,4aR,8aS)-4-(2-Bromophenyl)-2,7-dihydroxy-3,4,4a,5,6,8a-hexahydro-2H-chromen-8-yl)ethan-1-one

(3c)

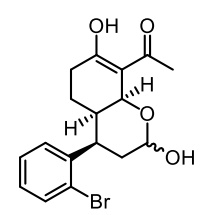

$\mathrm{dr}=1: 1$

Yield: $73 \%(80.3 \mathrm{mg})$

Physical State: pink amorphous

${ }^{1}$ H NMR (400 MHz, $\left.\mathbf{C D C l}_{3}\right) \delta 7.59(\mathrm{~d}, J=8.0 \mathrm{~Hz}, 2 \mathrm{H}), 7.34-7.28(\mathrm{~m}, 2 \mathrm{H}), 7.16-7.09(\mathrm{~m}, 4 \mathrm{H}), 5.53(\mathrm{~d}, J=3.2 \mathrm{~Hz}$, $1 \mathrm{H}), 5.14(\mathrm{~s}, 1 \mathrm{H}), 5.05(\mathrm{dd}, J=8.8,2.0 \mathrm{~Hz}, 1 \mathrm{H}), 4.55(\mathrm{~s}, 1 \mathrm{H}), 4.08(\mathrm{dt}, J=13.6,3.6 \mathrm{~Hz}, 1 \mathrm{H}), 3.63(\mathrm{dt}, J=13.6,3.6$ $\mathrm{Hz}, 1 \mathrm{H}), 2.41-2.34(\mathrm{~m}, 3 \mathrm{H}), 2.33(\mathrm{~s}, 3 \mathrm{H}), 2.33(\mathrm{~s}, 3 \mathrm{H}), 2.27(\mathrm{dd}, J=13.6,3.6 \mathrm{~Hz}, 1 \mathrm{H}), 2.23-2.16(\mathrm{~m}, 1 \mathrm{H})$, 2.15-2.07, (m, 2H), 2.06-1.97 (m, 2H), 1.91-1.82 (m, 3H), 1.75-1.71 (m, 1H), 1.11-1.05 (m, 1H)

${ }^{13}$ C NMR (100 MHz, CDCl $) \delta$ 200.4, 200.4, 185.5, 185.2, 140.5, 139.9, 133.4, 133.3, 128.9, 128.5, 128.3, 128.1, 127.3, 127.1, 125.1, 124.7, 108.9, 108.7, 96.2, 91.9, 72.1, 65.2, 42.4, 36.8, 36.3, 35.6, 32.3, 31.1, 31.0, 29.1, 24.0, $24.0,15.7,14.9$

IR (neat) $\vee 3414,2946,2891,1610,1470,1416,1363,1311,1265,1050,1022,1009,956,757,736 \mathrm{~cm}^{-1}$

HRMS (ESI) m/z: $[\mathrm{M}+\mathrm{Na}]^{+}$Calcd for $\mathrm{C}_{17} \mathrm{H}_{19}{ }^{79} \mathrm{BrO}_{4} \mathrm{Na}^{+}$389.0359; Found 389.0361

HRMS (ESI) m/z: [M+Na] ${ }^{+}$Calcd for $\mathrm{C}_{17} \mathrm{H}_{19}{ }^{81} \mathrm{BrO}_{4} \mathrm{Na}^{+}$391.0339; Found 391.0341

$[\alpha]_{\mathrm{D}}^{28}+15.7\left(c 0.700, \mathrm{CHCl}_{3}\right)$

$\mathbf{R}_{\mathbf{f}}(n$-Hexane: EtOAc $=3: 1$, color reagent: $p$-anisaldehyde): 0.10

(R)-3-(3-Acetyl-4-hydroxycyclohexa-1,3-dien-1-yl)-3-(2-bromophenyl)propanal (4c)<smiles>CC(=O)c1cc(CC=O)ccc1O</smiles>

Yield: 21\% (22.3 mg)

Physical State: yellow oil 
${ }^{1}$ H NMR (400 MHz, $\left.\mathbf{C D C l}_{3}\right) \delta 9.71(\mathrm{dd}, J=3.2,1.6 \mathrm{~Hz}, 1 \mathrm{H}), 7.59(\mathrm{dd}, J=8.0,1.2 \mathrm{~Hz}, 1 \mathrm{H}), 7.28(\mathrm{td}, J=8.0,1.6$ $\mathrm{Hz}, 1 \mathrm{H}), 7.20(\mathrm{dd}, J=8.0,1.6 \mathrm{~Hz}, 1 \mathrm{H}), 7.12(\mathrm{td}, J=8.0,1.6 \mathrm{~Hz}, 1 \mathrm{H}), 6.08(\mathrm{~s}, 1 \mathrm{H}), 4.52(\mathrm{t}, J=7.6 \mathrm{~Hz}, 1 \mathrm{H}), 2.90$ (ddd, $J=16.8,9.2,3.2 \mathrm{~Hz}, 1 \mathrm{H}), 2.82$ (ddd, $J=16.8,6.4,1.2 \mathrm{~Hz}, 1 \mathrm{H}), 2.50-2.41$ (m, 2H), 2.24 (dd, $J=16.4,7.6 \mathrm{~Hz}$, $1 \mathrm{H}), 2.15-2.06(\mathrm{~m}, 1 \mathrm{H}), 2.12(\mathrm{~s}, 3 \mathrm{H})$

${ }^{13}$ C NMR (100 MHz, CDCl $) \delta$ 200.7, 199.1, 179.4, 140.5, 133.9, 131.3, 128.7, 128.5, 127.9, 125.0, 119.3, 108.0, $46.9,44.5,35.0,25.7,19.7$

IR (neat) $v 3437,2956,2931,1721,1695,1607,1470,1435,1415,1359,1266,1220,1022,756,736 \mathrm{~cm}^{-1}$

HRMS (ESI) m/z: [M+Na] ${ }^{+}$Calcd for $\mathrm{C}_{17} \mathrm{H}_{18}{ }^{79} \mathrm{BrO}_{3} \mathrm{Na}^{+}$349.0434; Found 349.0433

HRMS (ESI) m/z: $[\mathrm{M}+\mathrm{Na}]^{+}$Calcd for $\mathrm{C}_{17} \mathrm{H}_{18}{ }^{81} \mathrm{BrO}_{3} \mathrm{Na}^{+}$351.0414; Found 349.0412

$[\alpha]_{\mathrm{D}}^{28}+112.0\left(c 0.570, \mathrm{CHCl}_{3}\right)$

$\mathbf{R}_{\mathbf{f}}(n$-Hexane: EtOAc $=3: 1$, color reagent: $p$-anisaldehyde): 0.35

1-((4R,4aR,8aS)-2,7-Dihydroxy-4-(4-methoxyphenyl)-3,4,4a,5,6,8a-hexahydro-2H-chromen-8-yl)ethan-1-one (3d)

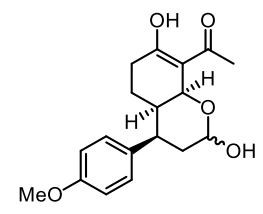

$\mathrm{dr}=1: 1$

Yield: $70 \%(66.9 \mathrm{mg})$

Physical State: pink amorphous

${ }^{1}$ H NMR (400 MHz, CDCl $)$ ) 7.11-7.07 (m, 4H), 6.90-6.87 (m, 4H), 5.51 (d, $\left.J=3.2 \mathrm{~Hz}, 1 \mathrm{H}\right), 5.29$ (d, $J=0.4 \mathrm{~Hz}$, $1 \mathrm{H}), 5.11(\mathrm{~s}, 1 \mathrm{H}), 4.99(\mathrm{dd}, J=8.8,2.0 \mathrm{~Hz}, 1 \mathrm{H}), 4.51(\mathrm{~s}, 1 \mathrm{H}), 3.80(\mathrm{~s}, 6 \mathrm{H}), 3.62(\mathrm{dt}, J=13.2,3.2 \mathrm{~Hz}, 1 \mathrm{H}), 3.21(\mathrm{dt}$, $J=13.2,3.2 \mathrm{~Hz}, 1 \mathrm{H}), 2.39-2.38(\mathrm{~m}, 1 \mathrm{H}), 2.35-2.33(\mathrm{~m}, 1 \mathrm{H}), 2.31(\mathrm{~s}, 3 \mathrm{H}), 2.31(\mathrm{~s}, 3 \mathrm{H}), 2.20-2.13(\mathrm{~m}, 4 \mathrm{H})$, 1.97-1.96 (m, 1H), 1.93-1.87 (m, 1H), 1.83-1.78 (m, 4H) 1.30-1.26 (m, 1H), 1.19-1.12 (m, 2H)

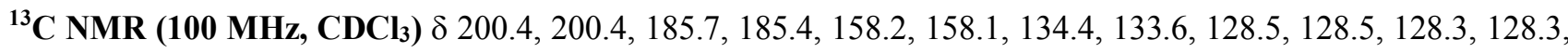
$113.8,113.8,113.7,113.7,108.9,108.6,96.4,92.0,72.1,65.3,55.2,55.2,42.0,40.0,39.4,36.3,32.2,31.2,31.1$, $29.1,23.9,23.9,15.4,14.6$

IR (neat) $v 3429,2937,1611,1517,1513,1416,1363,1251,1181,1109,1035,956,833,736 \mathrm{~cm}^{-1}$

HRMS (ESI) m/z: $[\mathrm{M}+\mathrm{Na}]^{+}$Calcd for $\mathrm{C}_{18} \mathrm{H}_{22} \mathrm{O}_{5} \mathrm{Na}^{+}$341.1360; Found 341.1365

$[\alpha]_{\mathrm{D}}^{28}+19.4\left(c 1.38, \mathrm{CHCl}_{3}\right)$

$\mathbf{R}_{\mathbf{f}}(n$-Hexane: EtOAc $=3: 1$, color reagent: $p$-anisaldehyde): 0.10

(R)-3-(3-Acetyl-4-hydroxycyclohexa-1,3-dien-1-yl)-3-(4-methoxyphenyl)propanal (4d)

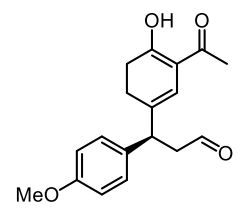


Yield: $21 \%(18.9 \mathrm{mg})$

Physical State: yellow oil

${ }^{1}$ H NMR (400 MHz, CDCl $) \delta 9.71(\mathrm{~s}, 1 \mathrm{H}), 7.14(\mathrm{~d}, J=8.4 \mathrm{~Hz}, 2 \mathrm{H}), 6.85(\mathrm{~d}, J=8.4 \mathrm{~Hz}, 2 \mathrm{H}), 6.09(\mathrm{~s}, 1 \mathrm{H}), 3.94(\mathrm{t}$, $J=7.6 \mathrm{~Hz}, 1 \mathrm{H}), 3.79$ (s, 3H), 2.95-2.87 (m, 2H), 2.54-2.49 (m, 1H), 2.43-2.38 (m, 2H), 2.16-2.11 (m, 1H), $2.13(\mathrm{~s}$, $3 \mathrm{H})$

${ }^{13}$ C NMR (100 MHz, CDCl $) \delta$ 201.3, 199.3, 178.9, 158.6, 133.4, 133.1, 128.6, 128.6, 117.7, 114.1, 114.1, 108.0, $55.2,47.3,45.1,35.1,24.9,19.6$

IR (neat) $v 2960,2934,1719,1609,1512,1441,1420,1359,1303,1251,1180,1033 \mathrm{~cm}^{-1}$

HRMS (ESI) m/z: [M+Na] ${ }^{+}$Calcd for $\mathrm{C}_{18} \mathrm{H}_{20} \mathrm{O}_{4} \mathrm{Na}^{+}$323.1254; Found 323.1254

$[\alpha]_{\mathrm{D}}^{26}+31.6\left(c 0.120, \mathrm{CHCl}_{3}\right)$

$\mathbf{R}_{\mathbf{f}}(n$-Hexane: EtOAc $=3: 1$, color reagent: $p$-anisaldehyde): 0.35

1-((4R,4aR,8aS)-4-(Furan-2-yl)-2,7-dihydroxy-3,4,4a,5,6,8a-hexahydro-2H-chromen-8-yl)ethan-1-one (3e)

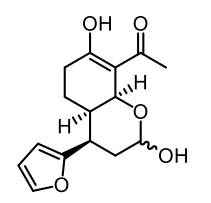

$\mathrm{dr}=1: 1$

Yield: $81 \%(67.3 \mathrm{mg})$

Physical State: pink amorphous

${ }^{1}$ H NMR (400 MHz, $\left.\mathbf{C D C l}_{3}\right) \delta 7.35(\mathrm{~s}, 1 \mathrm{H}), 6.33-6.31(\mathrm{~m}, 1 \mathrm{H}), 6.02(\mathrm{dd}, J=12.0,3.2 \mathrm{~Hz}, 1 \mathrm{H}), 5.46(\mathrm{~d}, J=3.2 \mathrm{~Hz}$, 1H), $5.05(\mathrm{~s}, 1 \mathrm{H}), 4.97(\mathrm{dd}, J=9.6,2.0 \mathrm{~Hz}, 1 \mathrm{H}), 4.47$ (s, 1H), 3.70 (dt, $J=13.2,4.0 \mathrm{~Hz}, 1 \mathrm{H}), 3.31$ (dt, $J=13.2,4.0$ $\mathrm{Hz}, 1 \mathrm{H}), 2.44-2.38(\mathrm{~m}, 3 \mathrm{H}), 2.30(\mathrm{~s}, 6 \mathrm{H}), 2.27-2.24(\mathrm{~m}, 1 \mathrm{H}), 2.12-2.00(\mathrm{~m}, 4 \mathrm{H}), 1.91-1.87(\mathrm{~m}, 2 \mathrm{H}), 1.85-1.80(\mathrm{~m}$, $2 \mathrm{H}), 1.78-1.71(\mathrm{~m}, 2 \mathrm{H}), 1.27-1,24(\mathrm{~m}, 1 \mathrm{H}), 1.19-109(\mathrm{~m}, 2 \mathrm{H})$

${ }^{13}$ C NMR (100 MHz, CDCl $) \delta$ 200.4, 200.3, 185.7 185.4, 156.6, 155.6, 141.5, 141.3, 110.0, 109.9, 108.8, 108.5, $105.5,105.1,95.8,91.6,71.5,64.4,37.2,37.2,36.6,32.0,31.2,31.1,31.0,27.9,24.0,23.9,16.2,15.5$

IR (neat) $v 3408,2943,1609,1507,1416,1362,1309,1259,1205,1133,1106,1011,958,922,737 \mathrm{~cm}^{-1}$

HRMS (ESI) m/z: [M+Na] ${ }^{+}$Calcd for $\mathrm{C}_{15} \mathrm{H}_{18} \mathrm{O}_{5} \mathrm{Na}^{+}$301.1047; Found 301.1047

$[\alpha]_{\mathrm{D}}{ }^{29}+57.7\left(c 1.82, \mathrm{CHCl}_{3}\right)$

$\mathbf{R}_{\mathbf{f}}(n$-Hexane: EtOAc $=3: 1$, color reagent: $p$-anisaldehyde): 0.10

(R)-3-(3-Acetyl-4-hydroxycyclohexa-1,3-dien-1-yl)-3-(furan-2-yl)propanal (4e)<smiles></smiles>

Yield: $19 \%(15.0 \mathrm{mg})$

Physical State: yellow oil 
${ }^{1}$ H NMR (400 MHz, CDCl $\left.)_{3}\right) \delta 9.68(\mathrm{~s}, 1 \mathrm{H}), 7.22-7.19(\mathrm{~m}, 1 \mathrm{H}), 6.24(\mathrm{t}, J=2.4 \mathrm{~Hz}, 1 \mathrm{H}), 6.05(\mathrm{~s}, 1 \mathrm{H}), 6.01-6.00(\mathrm{~m}$, $1 \mathrm{H}), 4.06(\mathrm{t}, J=7.6 \mathrm{~Hz}, 1 \mathrm{H}), 2.90(\mathrm{ddd}, J=16.8,6.8,2.0 \mathrm{~Hz}, 1 \mathrm{H}), 2.74(\mathrm{ddd}, J=16.8,7.6,2.0 \mathrm{~Hz}, 1 \mathrm{H}), 2.42-2.38$ (m, 2H), 2.18-2.14 (m, 2H), $2.03(\mathrm{~s}, 3 \mathrm{H})$

${ }^{13}$ C NMR (100 MHz, $\left.\mathbf{C D C l}_{3}\right) \delta$ 200.4, 199.3, 179.2, 154.6, 141.9, 130.1, 119.6, 110.3, 107.9, 106.2, 45.0, 40.2, $38.9,35.1,23.7$

IR (neat) $v 3429,2952,1723,1608,1505,1412,1264,1068,1011,738,705 \mathrm{~cm}^{-1}$

HRMS (ESI) m/z: [M+Na] ${ }^{+}$Calcd for $\mathrm{C}_{15} \mathrm{H}_{16} \mathrm{O}_{4} \mathrm{Na}^{+}$283.0941; Found 283.0945

$[\alpha]_{\mathrm{D}}^{24}-7.68\left(c 1.82, \mathrm{CHCl}_{3}\right)$

$\mathbf{R}_{\mathbf{f}}(n$-Hexane: EtOAc $=3: 1$, color reagent: $p$-anisaldehyde): 0.35

\subsection{Procedure of silane reduction}

\section{1-((4R,4aR,8aS)-7-Hydroxy-4-phenyl-3,4,4a,5,6,8a-hexahydro-2H-chromen-8-yl)ethan-1-one (6a)}

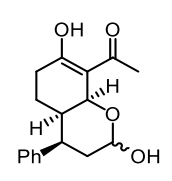

3a

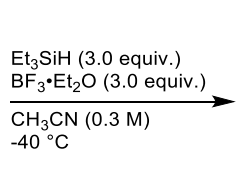

$\mathrm{Et}_{2} \mathrm{O}(3.0$ equiv.) $-40^{\circ} \mathrm{C}(0.3 \mathrm{M})$

Cat

To a solution of lactol 3a $(54 \mathrm{mg}, 0.19 \mathrm{mmol})$ in $\mathrm{CH}_{3} \mathrm{CN}(630 \mu \mathrm{L})$, triethylsilane $(90 \mu \mathrm{L}, 0.56 \mathrm{mmol})$ and boron trifluoride - diethyl ether complex $(71 \mu \mathrm{L}, 0.56 \mathrm{mmol})$ were added at $-40{ }^{\circ} \mathrm{C}$ under $\mathrm{Ar}$ atmosphere. After $25 \mathrm{~min}$, the reaction was quenched by the addition of sat. aq. $\mathrm{NaHCO}_{3}(1 \mathrm{~mL})$. Separated aqueous layer was extracted with EtOAc $(3 \mathrm{~mL} \times 3)$. The combined organic layers were washed with brine $(10 \mathrm{~mL})$, dried by $\mathrm{Na}_{2} \mathrm{SO}_{4}$, filtered and concentrated in vacuo. The residue was purified by flash chromatography on silica gel $(n$-Hexane: EtOAc $=12: 1)$ to give the corresponding ether (34 $\mathrm{mg}, 66 \%)$ as a white solid.

Yield: $66 \%(34 \mathrm{mg})$

Physical State: white solid (m.p. $117.0 \sim 119.0^{\circ} \mathrm{C}$ )

${ }^{1}$ H NMR (400 MHz, C $\left.\mathbf{C D}_{6}\right) \delta 7.22(\mathrm{t}, J=7.6 \mathrm{~Hz}, 2 \mathrm{H}), 7.12(\mathrm{~d}, J=7.6 \mathrm{~Hz}, 1 \mathrm{H}), 6.98(\mathrm{~d}, J=7.6 \mathrm{~Hz}, 2 \mathrm{H}), 3.87(\mathrm{ddd}$, $J=11.2,4,41.2 \mathrm{~Hz}, 1 \mathrm{H}), 3.82(\mathrm{~s}, 1 \mathrm{H}), 3.16(\mathrm{td}, J=11.2,2.4 \mathrm{~Hz}, 1 \mathrm{H}), 2.74(\mathrm{dt}, J=12.8,4.4 \mathrm{~Hz}, 1 \mathrm{H}), 2.12(\mathrm{~s}, 3 \mathrm{H})$, $2.08(\mathrm{ddd}, J=18.4,5.2,1.6 \mathrm{~Hz}, 1 \mathrm{H}), 1.80(\mathrm{td}, J=12.8,4.4 \mathrm{~Hz}, 1 \mathrm{H}), 1.74-1.65(\mathrm{~m}, 1 \mathrm{H}), 1.58(\mathrm{td}, J=12.8,5.2 \mathrm{~Hz}$ $1 \mathrm{H}), 1.43-1.39(\mathrm{~m}, 1 \mathrm{H}), 1.14-1.11(\mathrm{~m}, 1 \mathrm{H}), 0.84-0.79(\mathrm{~m}, 1 \mathrm{H})$

${ }^{13}$ C NMR (100 MHz, $\left.\mathbf{C D C l}_{3}\right) \delta$ 200.8, 185.1, 142.9, 128.5, 128.5, 127.4, 127.4, 126.5, 109.6, 74.4, 68.2, 44.3, 40.5, $31.3,24.9,23.9,15.5$

IR (neat) $v 2945,2852,1604,1416,1207,1082,1062,956,779,701 \mathrm{~cm}^{-1}$

HRMS (ESI) m/z: $[\mathrm{M}+\mathrm{Na}]^{+}$Calcd for $\mathrm{C}_{17} \mathrm{H}_{20} \mathrm{O}_{3} \mathrm{Na}^{+}$295.1305; Found 295.1305

$[\alpha]_{\mathrm{D}}^{26}+26.5\left(c 0.960, \mathrm{CHCl}_{3}\right)$

The enantiomeric ratio was determined by HPLC using CHIRALPACK ${ }^{\circledR}$ ID ( $n$-Hexane: $i$-PrOH $=49: 1$; flow rate $1.0 \mathrm{ml} / \mathrm{min}$, major isomer $t_{R}=14.8 \mathrm{~min}$, minor isomer $\left.t_{R}=10.9 \mathrm{~min}\right)(98 \%$ ee $)$

$\mathbf{R}_{\mathbf{f}}(n$-Hexane : EtOAc $=3: 1$, color reagent: $p$-anisaldehyde): 0.50 


\subsection{Wittig reaction}

\subsubsection{Wittig reaction without benzoic acid}

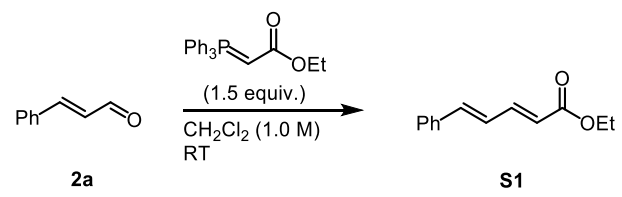

To a solution of $2 \mathbf{a}(40 \mathrm{mg}, 0.30 \mathrm{mmol})$ in $\mathrm{CH}_{2} \mathrm{Cl}_{2}(300 \mu \mathrm{L})$, Wittig reagent $(160 \mathrm{mg}, 0.45 \mathrm{mmol})$ was added at room temperature. After $1 \mathrm{~h}$, the reaction was quenched by the addition of sat. aq. $\mathrm{NaHCO}_{3}(1 \mathrm{~mL})$. Separated aqueous layer was extracted with $\mathrm{CH}_{2} \mathrm{Cl}_{2}(3 \mathrm{~mL} \times 3)$. The combined organic layers were washed with brine $(10$ $\mathrm{mL}$ ), dried by $\mathrm{Na}_{2} \mathrm{SO}_{4}$, filtered and concentrated in vacuo. The residue was purified by flash chromatography on silica gel $(n$-Hexane: EtOAc $=10: 1)$ to give $\mathbf{S 1}(E: Z=10: 1,54 \mathrm{mg}, 89 \%)$ as a colorless liquid.

\subsubsection{Wittig reaction in the presence of benzoic acid}

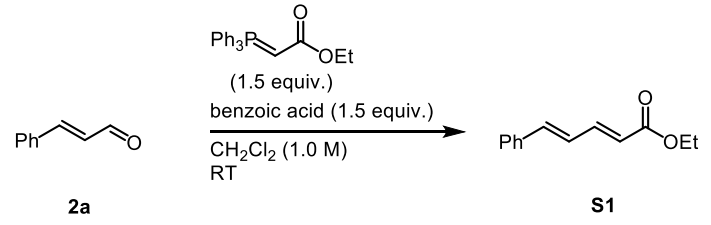

To a solution of $\mathbf{2 a}(40 \mathrm{mg}, 0.30 \mathrm{mmol})$ in $\mathrm{CH}_{2} \mathrm{Cl}_{2}(300 \mu \mathrm{L})$, benzoic acid $(55 \mathrm{mg}, 0.45 \mathrm{mmol})$ and Wittig reagent $(160 \mathrm{mg}, 0.45 \mathrm{mmol})$ were added at room temperature. After $15 \mathrm{~min}$, the reaction was quenched by the addition of sat. aq. $\mathrm{NaHCO}_{3}(1 \mathrm{~mL})$. Separated aqueous layer was extracted with $\mathrm{CH}_{2} \mathrm{Cl}_{2}(3 \mathrm{~mL} \times 3)$. The combined organic layers were washed with brine $(10 \mathrm{~mL})$, dried by $\mathrm{Na}_{2} \mathrm{SO}_{4}$, filtered and concentrated in vacuo. The residue was purified by flash chromatography on silica gel $(n$-Hexane: EtOAc $=10: 1)$ to give $\mathbf{S 1}(E: Z=10: 1,50 \mathrm{mg}, 82 \%)$ as a colorless liquid.

The value of $\mathrm{p} K_{\mathrm{a}}$ of phosponium ion $\left(\mathrm{Ph}_{3} \mathrm{P}^{+} \mathrm{CH}_{2} \mathrm{CO}_{2} \mathrm{Et}\right)$ is 8.5 (in DMSO) ${ }^{2}$ or 10.1 (calculation), ${ }^{3}$ while that of benzoic acid is 11.1 (in DMSO). ${ }^{4}$ We used $\mathrm{CH}_{2} \mathrm{Cl}_{2}$ as a solvent in the reaction. Based on these $\mathrm{p} K_{\mathrm{a}}$ values and difference of the solvent, Wittig reagent (ethyl 2-(triphenylphosphoranylidene)acetate) is thought to be not completely protonated by benzoic acid, which was supported by the above experiments.

\section{Ethyl (2E,4E)-5-phenylpenta-2,4-dienoate (S1)}<smiles>CCOC(=O)/C=C/C=C/c1ccccc1</smiles>

$E: Z=10: 1$

Physical State: colorless liquid

${ }^{1}$ H NMR (400 MHz, CDCl $) \delta$ 7.48-7.46 (m, 2H), $7.43(\mathrm{dd}, J=8.0,2.0 \mathrm{~Hz}, 1 \mathrm{H}), 7.38-7.29(\mathrm{~m}, 3 \mathrm{H})$, 6.89-6.87 (m, $2 \mathrm{H}), 5.99(\mathrm{~d}, J=15.2 \mathrm{~Hz}, 1 \mathrm{H}), 4.23(\mathrm{q}, J=7.2 \mathrm{~Hz}, 2 \mathrm{H}), 1.32(\mathrm{t}, J=7.2 \mathrm{~Hz}, 3 \mathrm{H})$ 


\subsection{Preparation of cyclic $\alpha$-acyl- $\alpha, \beta$-unsaturated ketone}

2.6.1 Preparation of 2-benzoylcyclohex-2-en-1-one (S3) and 2-(Pent-4-enoyl)cyclohex-2-en-1-one (S5) ${ }^{5}$

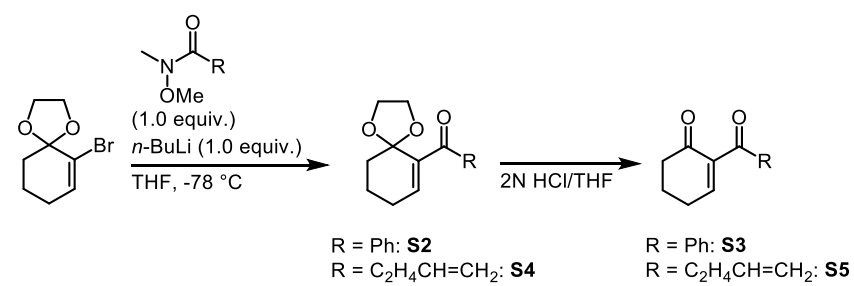

To a solution of vinyl bromide ${ }^{6}(0.35 \mathrm{~g}, 1.6 \mathrm{mmol})$ in THF (2.6 mL), $n$-BuLi (1.6 M/Hexane, $\left.1.2 \mathrm{~mL}, 1.9 \mathrm{mmol}\right)$ was added at $-78{ }^{\circ} \mathrm{C}$ under Ar atmosphere. After $1 \mathrm{~h}$, to a stirred solution, weinreb amide ${ }^{7}(0.19 \mathrm{~mL}, 1.3 \mathrm{mmol})$ in THF $(1.0 \mathrm{~mL})$ was added. After $1 \mathrm{~h}$, the reaction was quenched by the addition of sat. aq. $\mathrm{KH}_{2} \mathrm{PO}_{4}(6.0 \mathrm{~mL})$. Separated aqueous layer was extracted with diethyl ether $(10 \mathrm{~mL} \times 3)$. The combined organic layers were washed with brine $(20 \mathrm{~mL})$, dried over $\mathrm{MgSO}_{4}$, filtered and concentrated in vacuo. The residue was purified by flash chromatography on silica gel $(n$-Hexane: EtOAc $=15: 1)$ to give corresponding acetal $\mathbf{S 2}(0.24 \mathrm{~g}, 76 \%)$ as a yellow liquid.

To a stirred solution of acetal $\mathbf{S 2}(0.32 \mathrm{~g}, 1.3 \mathrm{mmol})$ in THF $(13 \mathrm{~mL}), 2 \mathrm{~N} \mathrm{HCl}(4.4 \mathrm{~mL})$ was added at room temperature. After $1 \mathrm{~h}$, the reaction mixture was quenched by the addition of sat. aq. $\mathrm{NaHCO}_{3}(10 \mathrm{~mL})$ at $0{ }^{\circ} \mathrm{C}$. Separated aqueous layer was extracted with EtOAc $(10 \mathrm{~mL} \times 3)$. The combined organic layers were washed with brine $(30 \mathrm{~mL})$, dried over $\mathrm{Na}_{2} \mathrm{SO}_{4}$, filtered and concentrated in vacuo. The residue was purified by flash chromatography on silica gel $(n$-Hexane: EtOAc $=3: 1)$ to give corresponding $\alpha, \beta$-unsaturated cyclic diketone $\mathbf{S 3}$ $(0.22 \mathrm{~g}, 86 \%)$ as a yellow liquid.

\section{Phenyl(1,4-dioxaspiro[4.5]dec-6-en-6-yl)methanone (S2)}<smiles></smiles>

Yield: $76 \%(0.24 \mathrm{~g})$

Physical State: colorless liquid

${ }^{1}$ H NMR (400 MHz, CDCl $) \delta 7.76(\mathrm{~d}, J=7.2 \mathrm{~Hz}, 2 \mathrm{H}), 7.48(\mathrm{t}, J=7.2 \mathrm{~Hz}, 1 \mathrm{H}), 7.38(\mathrm{t}, J=7.2 \mathrm{~Hz}, 2 \mathrm{H}), 6.36$ (t, $J$ $=4.0 \mathrm{~Hz}, 1 \mathrm{H}), 4.06-4.02(\mathrm{~m}, 2 \mathrm{H}), 3.93-3.90(\mathrm{~m}, 2 \mathrm{H}), 2.25-2.21(\mathrm{~m}, 2 \mathrm{H}), 1.89-1.83(\mathrm{~m}, 4 \mathrm{H})$

${ }^{13}$ C NMR (100 MHz, $\left.\mathbf{C D C l}_{3}\right) \delta 196.6,142.4,139.1,138.5,132.1,129.6,129.6,127.9,127.9,106.3,65.4,65.4$, 34.6, 25.1, 19.7

IR (neat) $v 3055,2984,2953,2897,1660,1447,1267,1177,1118,1073,1023,911,733,650 \mathrm{~cm}^{-1}$

HRMS (ESI) m/z: $[\mathrm{M}+\mathrm{H}]^{+}$Calcd for $\mathrm{C}_{15} \mathrm{H}_{16} \mathrm{O}_{3} \mathrm{H}^{+}$245.1173; Found 245.1174

\section{2-Benzoylcyclohex-2-en-1-one (S3)}<smiles>O=CC1=CCCCC1=O</smiles> 
Keto: Enol $=1: 2$

Yield: $86 \%(0.22 \mathrm{~g})$

Physical State: yellow liquid

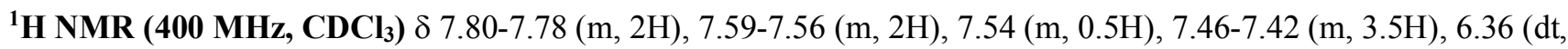
$J=10.0,1.2 \mathrm{~Hz}, 1 \mathrm{H}), 5.62(\mathrm{dt}, J=10.0,4.8 \mathrm{~Hz}, 1 \mathrm{H}), 2.64-2.56(\mathrm{~m}, 6 \mathrm{H}), 2.45-2.41(\mathrm{~m}, 2 \mathrm{H}), 2.17(\mathrm{~m}, 2 \mathrm{H})$

\section{1-(1,4-Dioxaspiro[4.5]dec-6-en-6-yl)pent-4-en-1-one (S4)}

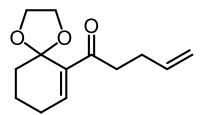

Yield: $60 \%(0.25 \mathrm{~g})$

Physical State: yellow liquid

${ }^{1}$ H NMR (400 MHz, CDCl $) \delta 6.88(\mathrm{t}, J=4.0 \mathrm{~Hz}, 1 \mathrm{H}), 5.87-5.77(\mathrm{~m}, 1 \mathrm{H}), 5.01(\mathrm{dt}, J=17.2,1.2 \mathrm{~Hz}, 1 \mathrm{H}), 4.94(\mathrm{dt}$, $J=10.4,1.2 \mathrm{~Hz}, 1 \mathrm{H}), 4.20-4.16(\mathrm{~m}, 2 \mathrm{H}), 4.02-3.98(\mathrm{~m}, 2 \mathrm{H}), 2.69$ (t, $J=7.6 \mathrm{~Hz}, 2 \mathrm{H}), 2.35-2.30$ (m, 2H), 2.25-2.21 (m, 2H), 1.77-1.73 (m, 4H)

${ }^{13}$ C NMR (100 MHz, $\left.\mathbf{C D C l}_{3}\right) \delta$ 200.0, 143.4, 139.3, 137.6, 114.8, 106.3, 65.0, 65.0, 39.1, 34.1, 27.9, 25.7, 19.7

IR (neat) $\vee 3055,2986,1421,1265,896,749,705 \mathrm{~cm}^{-1}$

HRMS (ESI) m/z: $[\mathrm{M}+\mathrm{Na}]^{+}$Calcd for $\mathrm{C}_{13} \mathrm{H}_{19} \mathrm{O}_{3} \mathrm{Na}^{+}$223.1329; Found 223.1321

\section{2-(Pent-4-enoyl)cyclohex-2-en-1-one (S5)}

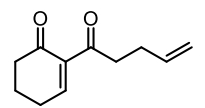

Keto: Enol $=5: 1$

Yield: $85 \%(0.17 \mathrm{~g})$

Physical State: yellow liquid

${ }^{1} \mathbf{H}$ NMR $\left(400 \mathrm{MHz}, \mathbf{C D C l}_{3}\right) \delta 7.60(\mathrm{t}, J=4.0 \mathrm{~Hz}, 1 \mathrm{H}), 6.26(\mathrm{~d}, J=10.0 \mathrm{~Hz}, 0.2 \mathrm{H}), 5.82(\mathrm{~m}, 1.2 \mathrm{H}), 5.59(\mathrm{dt}, J=$ 9.6, $4.0 \mathrm{~Hz}, 0.2 \mathrm{H}), 5.09-5.00(\mathrm{~m}, 1.4 \mathrm{H}), 4.96(\mathrm{dd}, J=10.0,1.2 \mathrm{~Hz}, 1 \mathrm{H}), 2.94(\mathrm{t}, J=7.2 \mathrm{~Hz}, 2 \mathrm{H}), 2.55-2.49$ (m, $4.8 \mathrm{H}), 2.37-2.32(\mathrm{~m}, 2.8 \mathrm{H}), 2.04(\mathrm{dt}, J=13.2,6.0 \mathrm{~Hz}, 2 \mathrm{H})$

${ }^{13}$ C NMR (100 MHz, CDCl $) \delta$ 200.3, 196.8, 156.0, 140.0, 137.3, 115.0, 41.8, 38.9, 27.8, 26.3, 22.2

IR (neat) $v 2932,1690,1639,1600,1359,1174,914,472,445,409 \mathrm{~cm}^{-1}$

HRMS (ESI) m/z: $[\mathrm{M}+\mathrm{H}]^{+}$Calcd for $\mathrm{C}_{11} \mathrm{H}_{14} \mathrm{O}_{2} \mathrm{H}^{+}$179.1067; Found 179.1064

\subsubsection{Preparation of 2-(5-(Trimethylsilyl)pent-4-ynoyl)cyclohex-2-en-1-one (S7)}

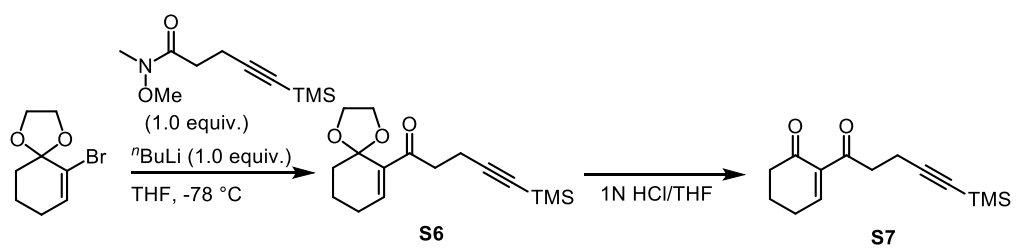


To a solution of vinyl bromide $(0.42 \mathrm{~g}, 1.9 \mathrm{mmol})$ in THF (2.8 mL), $n$-BuLi (1.2 M/Hexane, $1.6 \mathrm{~mL}, 1.9 \mathrm{mmol})$ was added at $-78{ }^{\circ} \mathrm{C}$ under Ar atmosphere. After $1 \mathrm{~h}$, to a stirred solution, weinreb amide $(0.41 \mathrm{~g}, 1.9 \mathrm{mmol})$ in THF $(1.0 \mathrm{~mL})$ was added. After $50 \mathrm{~min}$, the reaction was quenched by the addition of sat. aq. $\mathrm{KH}_{2} \mathrm{PO}_{4}(5.0 \mathrm{~mL})$. Separated aqueous layer was extracted with diethyl ether $(10 \mathrm{~mL} \times 3)$. The combined organic layers were washed with brine $(20 \mathrm{~mL})$, dried over $\mathrm{MgSO}_{4}$, filtered and concentrated in vacuo. The residue was purified by flash chromatography on silica gel $(n$-Hexane: EtOAc $=15: 1)$ to give corresponding acetal $\mathbf{S 6}(0.35 \mathrm{~g}, 64 \%)$ as a yellow liquid.

To a stirred solution of acetal $\mathbf{S 6}(0.35 \mathrm{~g}, 1.2 \mathrm{mmol})$ in THF $(12 \mathrm{~mL}), 1 \mathrm{~N} \mathrm{HCl}(6.1 \mathrm{~mL}, 6.1 \mathrm{mmol})$ was added at room temperature. After $1 \mathrm{~h}$, the reaction mixture was quenched by the addition of sat. aq. $\mathrm{NaHCO}_{3}(5.0 \mathrm{~mL})$ at 0

${ }^{\circ} \mathrm{C}$. Separated aqueous layer was extracted with EtOAc $(10 \mathrm{~mL} \times 3)$. The combined organic layers were washed with brine $(30 \mathrm{~mL})$, dried over $\mathrm{Na}_{2} \mathrm{SO}_{4}$, filtered and concentrated in vacuo. The residue was purified by flash chromatography on silica gel $(n$-Hexane: EtOAc $=10: 1)$ to give corresponding $\alpha, \beta$-unsaturated cyclic diketone $\mathbf{S} 7$ $(0.17 \mathrm{~g}, 97 \%)$ as a yellow liquid.

\section{1-(1,4-Dioxaspiro[4.5] dec-6-en-6-yl)-5-(trimethylsilyl)pent-4-yn-1-one (S6)}

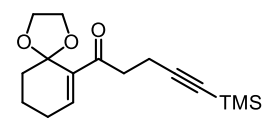

Yield: $64 \%(0.35 \mathrm{~g})$

Physical State: yellow liquid

${ }^{1}$ H NMR (400 MHz, CDCl $) \delta 6.94(\mathrm{t}, J=4.0 \mathrm{~Hz}, 1 \mathrm{H}), 4.21-4.18(\mathrm{~m}, 2 \mathrm{H}), 4.02-3.99(\mathrm{~m}, 2 \mathrm{H}), 2.88-2.84(\mathrm{~m}, 2 \mathrm{H})$, 2.50-2.46 (m, 2H), 2.26-2.23 (m, 2H), 1.76-1.75 (m, 4H), $0.12(\mathrm{~s}, 9 \mathrm{H})$

${ }^{13}$ C NMR (100 MHz, $\left.\mathbf{C D C l}_{3}\right) \delta$ 198.2, 144.1, 138.9, 106.4, 106.2, 84.6, 65.1, 65.1, 39.1, 34.2, 25.8, 19.7, 14.5, $0.07,0.07,0.07$

IR (neat) $v 2955,2898,2175,1687,1250,1189,1175,1110,1073,1030,954,843,761 \mathrm{~cm}^{-1}$

HRMS (ESI) m/z: $[\mathrm{M}+\mathrm{Na}]^{+}$Calcd for $\mathrm{C}_{16} \mathrm{H}_{24} \mathrm{O}_{3} \mathrm{SiNa}^{+}$315.1387; Found 315.1400

\section{2-(5-(Trimethylsilyl)pent-4-ynoyl)cyclohex-2-en-1-one (S7)}

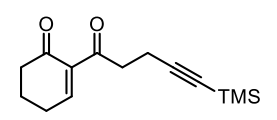

Yield: $97 \%(0.17 \mathrm{~g})$

Physical State: yellow liquid

${ }^{1}$ H NMR (400 MHz, $\left.\mathbf{C D C l}_{3}\right) \delta 7.65(\mathrm{t}, J=4.0 \mathrm{~Hz}, 1 \mathrm{H}), 3.10(\mathrm{t}, J=7.6 \mathrm{~Hz}, 2 \mathrm{H}), 2.56-2.49(\mathrm{~m}, 4 \mathrm{H}), 2.05(\mathrm{dt}, J=$ $12.8,6.0 \mathrm{~Hz}, 2 \mathrm{H}), 1.30-1.26(\mathrm{~m}, 1 \mathrm{H}), 0.88(\mathrm{~d}, J=6.8 \mathrm{~Hz}, 1 \mathrm{H}), 0.13(\mathrm{~s}, 9 \mathrm{H})$

${ }^{13}$ C NMR (100 MHz, $\left.\mathbf{C D C l}_{3}\right) \delta 198.6,196.7,156.6,139.5,106.0,84.7,41.8,38.8,26.4,22.1,14.5,0.07,0.07$, 0.07

IR (neat) $v 2958,2176,1692,1683,1601,1423,1360,1250,1174,843,760 \mathrm{~cm}^{-1}$ 


\subsubsection{Preparation of 2-acetylcyclopent-2-en-1-one (S8) and 2-acetylcyclohept-2-en-1-one (S9) ${ }^{8}$}

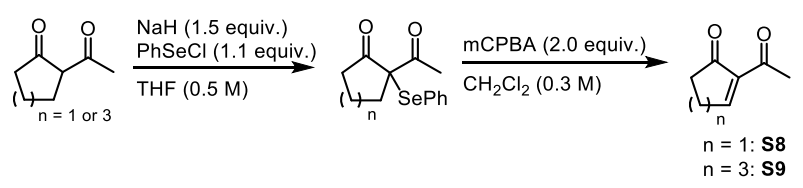

To a solution of $\mathrm{NaH}(60 \mathrm{wt} \%, 180 \mathrm{mg}, 4.5 \mathrm{mmol})$ in THF $(4.0 \mathrm{~mL})$, 2-acetylcyclopentan-1-one (370 $\mu \mathrm{L}, 3.0$ $\mathrm{mmol})$ in THF $(1.0 \mathrm{~mL})$ was added at $0{ }^{\circ} \mathrm{C}$ under Ar atmosphere. After $5 \mathrm{~min}$, to a stirred solution, $\mathrm{PhSeCl}(630 \mathrm{mg}$, $3.3 \mathrm{mmol})$ in THF $(1.0 \mathrm{~mL})$ was added. After $30 \mathrm{~min}$, the reaction was quenched by the addition of sat. aq. $\mathrm{NaHCO}_{3}(5.0 \mathrm{~mL})$. Separated aqueous layer was extracted with diethyl ether $(15 \mathrm{~mL} \times 3)$. The combined organic layers were washed with brine $(30 \mathrm{~mL})$, dried by $\mathrm{MgSO}_{4}$, filtered and concentrated in vacuo. The residue was roughly purified by flash chromatography on silica gel ( $n$-Hexane: EtOAc $=10: 1)$ to give 2-acetyl-2-(phenylselanyl)cyclopentan-1-one as a yellow liquid.

To a solution of 2-acetyl-2-(phenylselanyl)cyclopentan-1-one in $\mathrm{CH}_{2} \mathrm{Cl}_{2}(5.0 \mathrm{~mL}), m \mathrm{CPBA}$ (70 wt $\%, 1.6 \mathrm{~g}, 6.5$ mmol) was added at $0{ }^{\circ} \mathrm{C}$. After $1 \mathrm{~h}$, the reaction was quenched by the addition of sat. aq. $\mathrm{NaHCO}_{3}(5.0 \mathrm{~mL})$. Separated aqueous layer was extracted with $\mathrm{CH}_{2} \mathrm{Cl}_{2}(5.0 \mathrm{~mL} \times 3)$. The combined organic layers were washed with brine $(20 \mathrm{~mL})$, dried byNa2 $\mathrm{SO}_{4}$, filtered and concentrated in vacuo. The residue was purified by flash chromatography on silica gel ( $n$-Hexane: EtOAc $=16: 1)$ to give $\mathbf{S 8}(230 \mathrm{mg}, 61 \%)$ as a yellow liquid.

\section{2-Acetylcyclopent-2-en-1-one (S8)}<smiles>CC(C)=C1CCCC1=O</smiles>

Only keto form

Yield: $61 \%(230 \mathrm{mg})$

Physical State: yellow liquid

${ }^{1} \mathbf{H}$ NMR (400 MHz, CDCl$) \delta 8.39(\mathrm{t}, J=2.8 \mathrm{~Hz}, 1 \mathrm{H}), 2.75-2.72(\mathrm{~m}, 2 \mathrm{H}), 2.60-2.57(\mathrm{~m}, 2 \mathrm{H}), 2.48(\mathrm{~s}, 3 \mathrm{H})$

\section{2-Acetylcyclohept-2-en-1-one (S9)}<smiles>CC(=O)C1=C(C)CCCC1=O</smiles>

Keto: Enol $=2: 1$

Yield: $65 \%(130 \mathrm{mg})$

Physical State: yellow liquid

${ }^{1}$ H NMR (400 MHz, CDCl $) \delta 7.34(\mathrm{t}, J=7.0 \mathrm{~Hz}, 1 \mathrm{H}), 6.08(\mathrm{dt}, J=12.0,1.6 \mathrm{~Hz}, 0.5 \mathrm{H}), 5.68(\mathrm{dt}, J=12.0,5.2 \mathrm{~Hz}$, $0.5 \mathrm{H}), 2.69(\mathrm{t}, J=6.4 \mathrm{~Hz}, 2 \mathrm{H}), 2.55-2.51(\mathrm{~m}, 3 \mathrm{H}), 2.32(\mathrm{~s}, 3 \mathrm{H}), 2.29-2.25(\mathrm{~m}, 1 \mathrm{H}), 2.18(\mathrm{~s}, 1.5 \mathrm{H}), 2.02-1.96(\mathrm{~m}$, $1 \mathrm{H}), 1.89-1.83(\mathrm{~m}, 2 \mathrm{H}), 1.80-1.74(\mathrm{~m}, 2 \mathrm{H})$ 


\subsection{Typical procedure of the vinylogous Michael reaction/Wittig reaction}

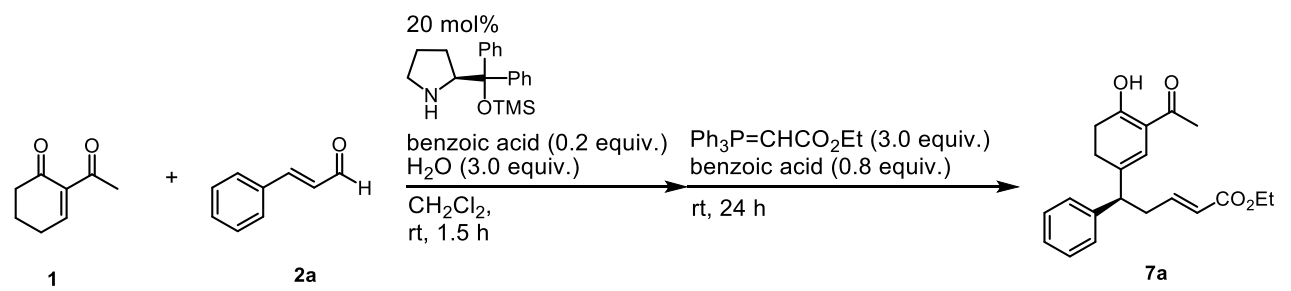

To a solution of ketone $\mathbf{1}(40 \mathrm{mg}, 0.30 \mathrm{mmol})$ and aldehyde $\mathbf{2 a}(63 \mathrm{mg}, 0.45 \mathrm{mmol})$ in $\mathrm{CH}_{2} \mathrm{Cl}_{2}(300 \mu \mathrm{L}), \mathrm{H}_{2} \mathrm{O}(16$ $\mu \mathrm{L}, 0.90 \mathrm{mmol})$, benzoic acid $(7.0 \mathrm{mg}, 0.060 \mathrm{mmol})$, and catalyst $(20 \mathrm{mg}, 0.060 \mathrm{mmol})$ were added at room temperature. After confirming the disappearance of aldehyde by TLC or ${ }^{1} \mathrm{H}-\mathrm{NMR}$, benzoic acid (29 $\mathrm{mg}, 0.24$ $\mathrm{mmol}$ ) and Wittig reagent $(310 \mathrm{mg}, 0.90 \mathrm{mmol})$ ware added to the reaction mixture. After $24 \mathrm{~h}$, the reaction was quenched by the addition of sat. aq. $\mathrm{NaHCO}_{3}(3 \mathrm{~mL})$. Separated aqueous layer was extracted with $\mathrm{CH}_{2} \mathrm{Cl}_{2}(5 \mathrm{~mL} \times$ 3). The combined organic layers were washed with brine $(10 \mathrm{~mL})$, dried by $\mathrm{Na}_{2} \mathrm{SO}_{4}$, filtered and concentrated in vacuo. The residue was purified by flash chromatography on silica gel $(n$-Hexane: EtOAc $=18: 1)$ to give compound 7a (71 mg, 70\%).

\subsection{Compound information}

\section{Ethyl $(R, E)$-5-(3-acetyl-4-hydroxycyclohexa-1,3-dien-1-yl)-5-phenylpent-2-enoate (7a)}<smiles>CCOC=CCc1ccccc1CCCOCC</smiles>

Yield: $70 \%(71.4 \mathrm{mg})$

Physical State: yellow oil

${ }^{1}$ H NMR (400 MHz, CDCl $) \delta 7.31(\mathrm{t}, J=7.2 \mathrm{~Hz}, 2 \mathrm{H}), 7.25-7.23(\mathrm{~m}, 1 \mathrm{H}), 7.22-7.20(\mathrm{~m}, 2 \mathrm{H}), 6.88(\mathrm{dt}, J=15.6$, $7.2 \mathrm{~Hz}, 1 \mathrm{H}), 6.13(\mathrm{~d}, J=1.2 \mathrm{~Hz}, 1 \mathrm{H}), 5.83(\mathrm{dt}, J=15.6,1.2 \mathrm{~Hz}, 1 \mathrm{H}), 4.16(\mathrm{q}, J=7.2 \mathrm{~Hz}, 2 \mathrm{H}), 3.50(\mathrm{t}, J=7.6 \mathrm{~Hz}$, $1 \mathrm{H}), 2.78(\mathrm{dtd}, J=15.6,7.2,1.2 \mathrm{~Hz}, 1 \mathrm{H}), 2.69$ (dtd, $J=15.6,7.2,1.2 \mathrm{~Hz}, 1 \mathrm{H}), 2.39$ (td, $J=7.6,1.6 \mathrm{~Hz}, 2 \mathrm{H}), 2.14$ (s, 3H), 2.13-2.10 (m, 2H), $1.26(\mathrm{t}, J=7.6 \mathrm{~Hz}, 3 \mathrm{H})$

${ }^{13}$ C NMR (100 MHz, $\left.\mathbf{C D C l}_{3}\right) \delta$ 199.5, 178.7, 166.4, 146.9, 142.0, 133.3, 128.6, 128.6, 127.6, 127.6, 126.8, 122.7, $117.9,108.1,60.3,51.0,35.7,35.2,24.6,19.6,14.2$

IR (neat) $v 2981,1717,1603,1493,1368,1271,1206,1038,981,738,703 \mathrm{~cm}^{-1}$

HRMS (ESI) m/z: $[\mathrm{M}+\mathrm{Na}]^{+}$Calcd for $\mathrm{C}_{21} \mathrm{H}_{24} \mathrm{O}_{4} \mathrm{Na}^{+}$363.1567; Found 363.1569 $[\alpha]_{\mathrm{D}}^{27}+17.3\left(c 1.23, \mathrm{CHCl}_{3}\right)$

The enantiomeric ratio was determined by HPLC using CHIRALPACK ${ }^{\circledR}$ IC $(n$-Hexane: $i$-PrOH $=19: 1$; flow rate $1.0 \mathrm{ml} / \mathrm{min}$, major isomer $t_{R}=21.7 \mathrm{~min}$, minor isomer $\left.t_{R}=19.9 \mathrm{~min}\right)(98 \% e e)$.

$\mathbf{R}_{\mathbf{f}}(n$-Hexane: EtOAc $=3: 1$, color reagent: $p$-anisaldehyde) $: 0.41$ 


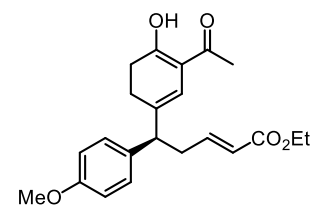

Yield: $70 \%(77.7 \mathrm{mg})$

Physical State: yellow oil

${ }^{1} \mathbf{H}$ NMR (400 MHz, $\left.\mathbf{C D C l}_{3}\right) \delta 7.11(\mathrm{~d}, J=8.8 \mathrm{~Hz}, 2 \mathrm{H}), 6.90-6.82(\mathrm{~m}, 3 \mathrm{H}), 6.10(\mathrm{~d}, J=1.2 \mathrm{~Hz}, 1 \mathrm{H}), 5.81(\mathrm{~d}, J=$ $15.6 \mathrm{~Hz}, 1 \mathrm{H}), 4.15$ (q, $J=7.2 \mathrm{~Hz}, 2 \mathrm{H}), 3.78(\mathrm{~s}, 3 \mathrm{H}), 3.44$ (t, $J=7.6 \mathrm{~Hz}, 1 \mathrm{H}), 2.74$ (dtd, $J=15.2,7.6,1.2 \mathrm{~Hz}, 1 \mathrm{H})$, $2.64(\mathrm{dtd}, J=15.2,7.6,1.2 \mathrm{~Hz}, 1 \mathrm{H}), 2.38(\mathrm{td}, J=7.2,1.2 \mathrm{~Hz}, 2 \mathrm{H}), 2.12-2.08(\mathrm{~m}, 5 \mathrm{H}), 1.25(\mathrm{t}, J=7.2 \mathrm{~Hz}, 3 \mathrm{H})$

${ }^{13}$ C NMR (100 MHz, CDCl $) \delta$ 199.5, 178.5, 166.3, 158.3, 147.0, 134.0, 133.7, 128.6, 128.6, 122.6, 117.6, 113.9, $113.9,108.1,60.2,55.2,50.2,35.9,35.224 .5,19.6,14.2$

IR (neat) $v 2936,1716,1653,1610,1509,1368,1251,1180,1113,1036,835,736 \mathrm{~cm}^{-1}$

HRMS (ESI) m/z: $[\mathrm{M}+\mathrm{Na}]^{+}$Calcd for $\mathrm{C}_{22} \mathrm{H}_{26} \mathrm{O}_{5} \mathrm{Na}^{+}$393.1672; Found 393.1678

$[\alpha]_{\mathrm{D}}^{27}+25.2\left(c 1.00, \mathrm{CHCl}_{3}\right)$

The enantiomeric ratio was determined by HPLC using CHIRALPACK ${ }^{\circledR}$ IA $(n$-Hexane: $i$-PrOH $=99: 1$; flow rate $1.0 \mathrm{ml} / \mathrm{min}$, major isomer $\mathrm{t}_{\mathrm{R}}=23.3 \mathrm{~min}$, minor isomer $\left.\mathrm{t}_{\mathrm{R}}=26.0 \mathrm{~min}\right)(>99 \% e e)$.

$\mathbf{R}_{\mathbf{f}}(n$-Hexane: EtOAc $=3: 1$, color reagent: $p$-anisaldehyde): 0.33

Ethyl (R,E)-5-(3-acetyl-4-hydroxycyclohexa-1,3-dien-1-yl)-5-(p-tolyl)pent-2-enoate (7c)

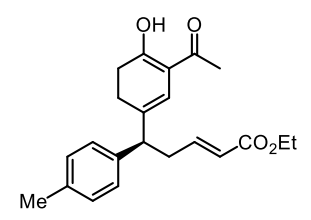

Yield: $66 \%$ (70.2 mg)

Physical State: yellow oil

${ }^{1}$ H NMR (400 MHz, CDCl $\left.)_{3}\right) \delta$ 7.12-7.07 (m, 4H), $6.87(\mathrm{dt}, J=15.6,7.2 \mathrm{~Hz}, 1 \mathrm{H}), 6.12(\mathrm{~d}, J=1.2 \mathrm{~Hz}, 1 \mathrm{H}), 5.83(\mathrm{dt}$, $J=15.6,1.6 \mathrm{~Hz}, 1 \mathrm{H}), 4.15(\mathrm{q}, J=7.2 \mathrm{~Hz}, 2 \mathrm{H}), 3.47$ (t, $J=7.6 \mathrm{~Hz}, 1 \mathrm{H}), 2.75(\mathrm{dtd}, J=14.8,7.6,1.2 \mathrm{~Hz}, 1 \mathrm{H}), 2.67$ (dtd, $J=14.8,7.6,1.2 \mathrm{~Hz}, 1 \mathrm{H}), 2.37(\mathrm{~m}, 2 \mathrm{H}), 2.32(\mathrm{~s}, 3 \mathrm{H}), 2.13-2.09(\mathrm{~m}, 5 \mathrm{H}), 1.26(\mathrm{t}, J=7.2 \mathrm{~Hz}, 3 \mathrm{H})$

${ }^{13}$ C NMR (100 MHz, $\left.\mathbf{C D C l}_{3}\right) \delta$ 199.5, 178.5, 166.3, 147.0, 139.0, 136.3, 133.5, 129.3, 129.3, 127.5, 127.5, 122.6, $117.7,108.1,60.2,50.6,35.8,35.2,24.5,21.0,19.6,14.2$

IR (neat) $\vee 3446,1717,1652,1617,1269,1203,1156 \mathrm{~cm}^{-1}$

HRMS (ESI) m/z: $[\mathrm{M}+\mathrm{Na}]^{+}$Calcd for $\mathrm{C}_{22} \mathrm{H}_{26} \mathrm{O}_{4} \mathrm{Na}^{+}$377.1723; Found 377.1729

$[\alpha]_{\mathrm{D}}^{28}+26.5\left(c 0.930, \mathrm{CHCl}_{3}\right)$

The enantiomeric ratio was determined by HPLC using CHIRALPACK ${ }^{\circledR} \mathrm{IC}(n$-Hexane: $i$-PrOH $=49: 1$; flow rate $1.0 \mathrm{ml} / \mathrm{min}$, major isomer $\mathrm{t}_{\mathrm{R}}=35.7 \mathrm{~min}$, minor isomer $\left.\mathrm{t}_{\mathrm{R}}=32.1 \mathrm{~min}\right)(98 \%$ ee $)$.

$\mathbf{R}_{\mathbf{f}}(n$-Hexane: EtOAc $=3: 1$, color reagent: $p$-anisaldehyde): 0.54 
<smiles>CCOC=CCC(c1ccc(F)cc1)c1ccc(O)c(C)c1</smiles>

Yield: $74 \%$ (79.6 mg)

Physical State: yellow oil

${ }^{1}$ H NMR (400 MHz, CDCl $) \delta$ 7.17-7.14 (m, 2H), $7.00(\mathrm{t}, J=8.8 \mathrm{~Hz}, 2 \mathrm{H}), 6.84(\mathrm{dt}, J=15.6,6.8 \mathrm{~Hz}, 1 \mathrm{H}), 6.12(\mathrm{~s}$, $1 \mathrm{H}), 5.81(\mathrm{~d}, J=15.6 \mathrm{~Hz}, 1 \mathrm{H}), 4.16(\mathrm{q}, J=7.2 \mathrm{~Hz}, 2 \mathrm{H}), 3.48(\mathrm{t}, J=7.2 \mathrm{~Hz}, 1 \mathrm{H}), 2.76(\mathrm{dt}, J=14.8,7.2 \mathrm{~Hz}, 1 \mathrm{H})$, $2.65(\mathrm{dt}, J=14.8,7.2 \mathrm{~Hz}, 1 \mathrm{H}), 2.40(\mathrm{td}, J=7.6,2.4 \mathrm{~Hz}, 2 \mathrm{H}), 2.14$ (s, 3H), 2.10 (t, $J=7.6 \mathrm{~Hz}, 2 \mathrm{H}), 1.27$ (t, $J=7.2$ $\mathrm{Hz}, 3 \mathrm{H})$

${ }^{13}$ CNMR (100 MHz, $\left.\mathbf{C D C l}_{3}\right) \delta$ 199.3, 178.8, 166.2, 162.9, 160.4, 146.5, 137.7 (d, $\left.J_{\text {C-F }}=3.2 \mathrm{~Hz}\right), 133.1,129.0$, $122.9,118.3,115.5,115.3,108.0,60.3,50.3,35.8,35.1,24.6,19.6,14.2$

IR (neat) $\vee 3438,1715,1651,1604,1509,1369,1272,1223,1160 \mathrm{~cm}^{-1}$

HRMS (ESI) m/z: $[\mathrm{M}+\mathrm{Na}]^{+}$Calcd for $\mathrm{C}_{21} \mathrm{H}_{23} \mathrm{FO}_{4} \mathrm{Na}^{+}$381.1473; Found 381.1473

$[\alpha]_{\mathrm{D}}^{27}+14.5\left(c 0.750, \mathrm{CHCl}_{3}\right)$

The enantiomeric ratio was determined by HPLC using CHIRALPACK ${ }^{\circledR}$ IC $(n$-Hexane: $i$-PrOH $=49: 1$; flow rate $1.0 \mathrm{ml} / \mathrm{min}$, major isomer $t_{\mathrm{R}}=45.4 \mathrm{~min}$, minor isomer $\left.\mathrm{t}_{\mathrm{R}}=41.9 \mathrm{~min}\right)(98 \%$ ee $)$.

$\mathbf{R}_{\mathbf{f}}(n$-Hexane: EtOAc $=3: 1$, color reagent: $p$-anisaldehyde): 0.36

Ethyl (R,E)-5-(3-acetyl-4-hydroxycyclohexa-1,3-dien-1-yl)-5-(4-chlorophenyl)pent-2-enoate (7e)

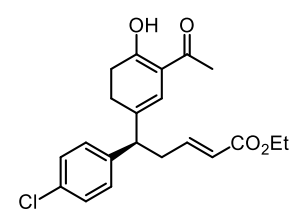

Yield: $73 \%(82.1 \mathrm{mg})$

Physical State: yellow oil

${ }^{1}$ H NMR (400MHz, CDCl $) \delta 7.28(\mathrm{~d}, J=8.4 \mathrm{~Hz}, 2 \mathrm{H}), 7.14(\mathrm{~d}, J=8.4 \mathrm{~Hz}, 2 \mathrm{H}), 6.84(\mathrm{dt}, J=15.6,7.6 \mathrm{~Hz}, 1 \mathrm{H})$, $6.12(\mathrm{~s}, 1 \mathrm{H}), 5.82(\mathrm{~d}, J=15.6 \mathrm{~Hz}, 1 \mathrm{H}), 4.16(\mathrm{q}, J=7.2 \mathrm{~Hz}, 2 \mathrm{H}), 3.48(\mathrm{t}, J=7.6 \mathrm{~Hz}, 1 \mathrm{H}), 2.75$ (dt, $J=15.2,7.6 \mathrm{~Hz}$, 1H), $2.65(\mathrm{dt}, J=14.8,7.6 \mathrm{~Hz}, 1 \mathrm{H}), 2.40(\mathrm{td}, J=7.6,2.0 \mathrm{~Hz}, 2 \mathrm{H}), 2.14(\mathrm{~s}, 3 \mathrm{H}), 2.10(\mathrm{t}, J=7.6 \mathrm{~Hz}, 2 \mathrm{H}), 1.27(\mathrm{t}, J=$ $7.2 \mathrm{~Hz}, 3 \mathrm{H})$

${ }^{13}$ C NMR (100 MHz, CDCl $) \delta$ 199.2, 178.9, 166.2, 146.2, 140.5, 132.7, 132.5, 129.0, 129.0, 128.7, 128.7, 123.0, $118.2,108.0,60.3,50.4,35.6,35.1,24.5,19.6,14.2$

IR (neat) $v 1716,1653,1609,1491,1406,1270,1205,1092,1014 \mathrm{~cm}^{-1}$

HRMS (ESI) m/z: $[\mathrm{M}+\mathrm{Na}]^{+}$Calcd for $\mathrm{C}_{21} \mathrm{H}_{23}{ }^{35} \mathrm{ClO}_{4} \mathrm{Na}^{+}$397.1177; Found 397.1180

HRMS (ESI) m/z: $[\mathrm{M}+\mathrm{Na}]^{+}$Calcd for $\mathrm{C}_{21} \mathrm{H}_{23}{ }^{37} \mathrm{ClO}_{4} \mathrm{Na}^{+} 399.1148$; Found 399.1151

$[\alpha]_{\mathrm{D}}^{23}+23.0\left(c 1.15, \mathrm{CHCl}_{3}\right)$

The enantiomeric ratio was determined by HPLC using CHIRALPACK ${ }^{\circledR}$ IC $(n$-Hexane: $i$-PrOH $=49: 1$; flow rate 
$1.0 \mathrm{ml} / \mathrm{min}$, major isomer $\mathrm{t}_{\mathrm{R}}=50.8 \mathrm{~min}$, minor isomer $\left.\mathrm{t}_{\mathrm{R}}=45.3 \mathrm{~min}\right)(98 \% \mathrm{ee})$.

$\mathbf{R}_{\mathbf{f}}(n$-Hexane: EtOAc $=3: 1$, color reagent: $p$-anisaldehyde $): 0.39$

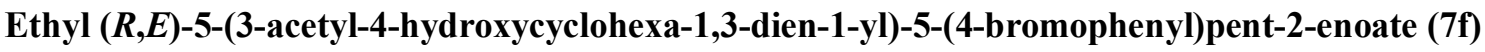

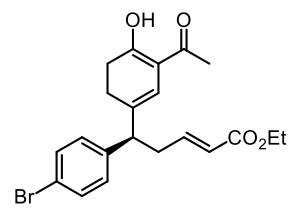

Yield: $76 \%(95.6 \mathrm{mg})$

Physical State: yellow oil

${ }^{1}$ H NMR (400 MHz, CDCl $) \delta 7.43(\mathrm{~d}, J=8.4 \mathrm{~Hz}, 2 \mathrm{H}), 7.08(\mathrm{~d}, J=8.4 \mathrm{~Hz}, 2 \mathrm{H}), 6.84(\mathrm{dt}, J=15.6,7.2 \mathrm{~Hz}, 1 \mathrm{H})$, $6.12(\mathrm{~s}, 1 \mathrm{H}), 5.82(\mathrm{~d}, J=15.6 \mathrm{~Hz}, 1 \mathrm{H}), 4.16(\mathrm{q}, J=7.2 \mathrm{~Hz}, 2 \mathrm{H}), 3.46(\mathrm{t}, J=7.6,1 \mathrm{H}), 2.75(\mathrm{dt}, J=14.8,7.6 \mathrm{~Hz}$, $1 \mathrm{H}), 2.65(\mathrm{dt}, J=14.8,7.6 \mathrm{~Hz}, 1 \mathrm{H}), 2.39(\mathrm{td}, J=7.6,2.0 \mathrm{~Hz}, 2 \mathrm{H}), 2.14(\mathrm{~s}, 3 \mathrm{H}), 2.09(\mathrm{t}, J=7.6 \mathrm{~Hz}, 2 \mathrm{H}), 1.27(\mathrm{t}, J=$ $7.6 \mathrm{~Hz}, 3 \mathrm{H})$

${ }^{13}$ C NMR (100 MHz, CDCl 3$) \delta 199.2,178.9,166.2,146.2,141.0,132.6,131.7,131.7,129.4,129.4,123.1,120.6$, $118.3,108.0,60.3,50.5,35.6,35.1,24.6,19.7,14.2$

IR (neat) $v 1717,1654,1609,1488,1401,1367,1315,1270,1205,1157,1010 \mathrm{~cm}^{-1}$

HRMS (ESI) m/z: $[\mathrm{M}+\mathrm{Na}]^{+}$Calcd for $\mathrm{C}_{21} \mathrm{H}_{23}{ }^{79} \mathrm{BrO}_{4} \mathrm{Na}^{+} 441.0672$; Found 441.0679

HRMS (ESI) m/z: $[\mathrm{M}+\mathrm{Na}]^{+}$Calcd for $\mathrm{C}_{21} \mathrm{H}_{23}{ }^{81} \mathrm{BrO}_{4} \mathrm{Na}^{+} 443.0652$; Found 441.0661

$[\alpha]_{\mathrm{D}}^{27}+33.1\left(c 0.990, \mathrm{CHCl}_{3}\right)$

The enantiomeric ratio was determined by HPLC using CHIRALPACK ${ }^{\circledR}$ IC ( $n$-Hexane: $i$-PrOH $=49: 1$; flow rate $1.0 \mathrm{ml} / \mathrm{min}$, major isomer $t_{R}=51.6 \mathrm{~min}$, minor isomer $\left.t_{R}=48.4 \mathrm{~min}\right)(>99 \%$ ee $)$.

$\mathbf{R}_{\mathbf{f}}(n$-Hexane: EtOAc $=3: 1$, color reagent: $p$-anisaldehyde): 0.37

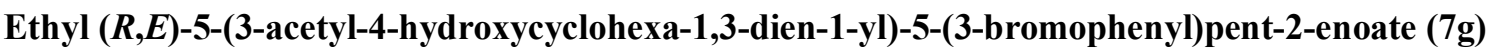<smiles>CCO/C=C/CC(C1=CC(O)=C(C(C)=O)CC1)c1cccc(Br)c1</smiles>

Yield: $73 \%(82.9 \mathrm{mg})$

Physical State: yellow oil

${ }^{1}$ H NMR (400 MHz, CDCl $) \delta$ 7.38-7.34 (m, 2H), $7.18(\mathrm{dd}, J=7.2,7.2 \mathrm{~Hz}, 1 \mathrm{H}), 7.15-7.12(\mathrm{~m}, 1 \mathrm{H}), 6.84(\mathrm{dt}, J=$ 15.6, $7.2 \mathrm{~Hz}, 1 \mathrm{H}), 6.13(\mathrm{~d}, J=0.80 \mathrm{~Hz}, 1 \mathrm{H}), 5.83(\mathrm{dt}, J=15.6,1.2 \mathrm{~Hz}, 1 \mathrm{H}), 4.16(\mathrm{q}, J=7.2 \mathrm{~Hz}, 2 \mathrm{H}), 3.47(\mathrm{t}, J=$ $7.6 \mathrm{~Hz}, 1 \mathrm{H}), 2.76(\mathrm{dtd}, J=15.6,7.6,1.2 \mathrm{~Hz}, 1 \mathrm{H}), 2.66(\mathrm{dtd}, J=15.6,7.6,1.2 \mathrm{~Hz}, 1 \mathrm{H}), 2.40(\mathrm{td}, J=7.6,1.6 \mathrm{~Hz}$, 2H), $2.14(\mathrm{~s}, 3 \mathrm{H}), 2.10(\mathrm{t}, J=7.6 \mathrm{~Hz}, 2 \mathrm{H}), 1.27(\mathrm{t}, J=7.2 \mathrm{~Hz}, 3 \mathrm{H})$

${ }^{13}$ C NMR (100 MHz, CDCl $) \delta 199.2,179.0,166.2,146.2,144.5,132.3,130.6,130.2,130.0,126.4,123.1,122.8$, $118.6,108.0,60.3,50.8,35.5,35.1,24.5,19.7,14.2$

IR (neat) v 3449, 1715, 1652, 1609, 1593, 1568, 1425, 1368, 1271, 1206, $1159 \mathrm{~cm}^{-1}$ 
HRMS (ESI) m/z: [M+Na] ${ }^{+}$Calcd for $\mathrm{C}_{21} \mathrm{H}_{23}{ }^{79} \mathrm{BrO}_{4} \mathrm{Na}^{+}$441.0672; Found 441.0674

HRMS (ESI) m/z: $[\mathrm{M}+\mathrm{Na}]^{+} \mathrm{Calcd}$ for $\mathrm{C}_{21} \mathrm{H}_{23}{ }^{81} \mathrm{BrO}_{4} \mathrm{Na}^{+} 443.0652$; Found 443.0658

$[\alpha]_{\mathrm{D}}^{25}+23.9\left(c 0.980, \mathrm{CHCl}_{3}\right)$

The enantiomeric ratio was determined by HPLC using CHIRALPACK ${ }^{\circledR}$ IC $(n$-Hexane: $i$-PrOH $=49: 1$; flow rate $1.0 \mathrm{ml} / \mathrm{min}$, major isomer $t_{R}=49.3 \mathrm{~min}$, minor isomer $\left.t_{R}=42.3 \mathrm{~min}\right)(>99 \%$ ee $)$

$\mathbf{R}_{\mathbf{f}}(n$-Hexane: EtOAc $=3: 1$, color reagent: $p$-anisaldehyde): 0.37

\section{Ethyl $(R, E)$-5-(3-acetyl-4-hydroxycyclohexa-1,3-dien-1-yl)-5-(2-bromophenyl)pent-2-enoate (7h)}

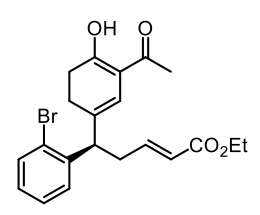

Yield: $66 \%(83.1 \mathrm{mg})$

Physical State: yellow oil

${ }^{1}$ H NMR (400 MHz, $\left.\mathbf{C D C l}_{3}\right) \delta 7.56(\mathrm{dd}, J=8.0,1.2 \mathrm{~Hz}, 1 \mathrm{H}), 7.28(\mathrm{td}, J=8.0,0.8 \mathrm{~Hz}, 1 \mathrm{H}), 7.21(\mathrm{dd}, J=7.6,2.0$ $\mathrm{Hz}, 1 \mathrm{H}), 7.09$ (ddd, $J=8.0,7.2,1.6 \mathrm{~Hz}, 1 \mathrm{H}), 6.91(\mathrm{dt}, J=15.6,7.2 \mathrm{~Hz}, 1 \mathrm{H}), 6.13(\mathrm{~d}, J=0.8 \mathrm{~Hz}, 1 \mathrm{H}), 5.84(\mathrm{dt}, J=$ 15.6, $2.0 \mathrm{~Hz}, 1 \mathrm{H}), 4.16(\mathrm{q}, J=7.2 \mathrm{~Hz}, 2 \mathrm{H}), 4.06(\mathrm{t}, J=7.2 \mathrm{~Hz}, 1 \mathrm{H}), 2.79(\mathrm{~m}, 1 \mathrm{H}), 2.68-2.61(\mathrm{~m}, 1 \mathrm{H}), 2.45-2.37(\mathrm{~m}$, $2 \mathrm{H}), 2.20-2.14(\mathrm{~m}, 1 \mathrm{H}), 2.11-2.08(\mathrm{~m}, 1 \mathrm{H}), 2.11(\mathrm{~s}, 3 \mathrm{H}), 1.27(\mathrm{t}, J=7.2 \mathrm{~Hz}, 3 \mathrm{H})$

${ }^{13}$ C NMR (100 MHz, CDCl $) \delta$ 199.4, 178.9, 166.3, 146.4, 141.1, 133.2, 131.7, 128.4, 128.2, 127.7, 125.4, 123.0, $118.9,108.1,60.3,49.0,35.8,35.1,25.5,19.7,14.2$

IR (neat) $v$ 3055, 2985, 1715, 1654, 1609, 1469, 1420, 1368, 1265, 1206, $741 \mathrm{~cm}^{-1}$

HRMS (ESI) m/z: $[\mathrm{M}+\mathrm{Na}]^{+}$Calcd for $\mathrm{C}_{21} \mathrm{H}_{23}{ }^{79} \mathrm{BrO}_{4} \mathrm{Na}^{+} 441.0672$; Found 441.0671

HRMS (ESI) m/z: $[\mathrm{M}+\mathrm{Na}]^{+}$Calcd for $\mathrm{C}_{21} \mathrm{H}_{23}{ }^{81} \mathrm{BrO}_{4} \mathrm{Na}^{+} 443.0652$; Found 441.0652 $[\alpha]_{\mathrm{D}}^{28}+24.6\left(c 1.45, \mathrm{CHCl}_{3}\right)$

The enantiomeric ratio was determined by HPLC using CHIRALPACK ${ }^{\circledR}$ IC $(n$-Hexane: $i$-PrOH $=49: 1$; flow rate $1.0 \mathrm{ml} / \mathrm{min}$, major isomer $\mathrm{t}_{\mathrm{R}}=38.9 \mathrm{~min}$, minor isomer $\left.\mathrm{t}_{\mathrm{R}}=35.3 \mathrm{~min}\right)(>99 \% e e)$.

$\mathbf{R}_{\mathbf{f}}(n$-Hexane: $\mathrm{EtOAc}=3: 1$, color reagent: $p$-anisaldehyde): 0.35

Ethyl (R,E)-5-(3-acetyl-4-hydroxycyclohexa-1,3-dien-1-yl)-5-(naphthalen-2-yl)pent-2-enoate (7i)

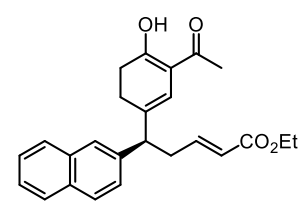

Yield: $79 \%(93.3 \mathrm{mg})$

Physical State: yellow oil

${ }^{1}$ H NMR (400 MHz, $\left.\mathbf{C D C l}_{3}\right) \delta$ 7.83-7.78 (m, 3H), $7.65(\mathrm{~s}, 1 \mathrm{H}), 7.50-7.44(\mathrm{~m}, 2 \mathrm{H}), 7.34(\mathrm{dd}, J=8.4,1.6 \mathrm{~Hz}, 1 \mathrm{H})$, $6.92(\mathrm{dt}, J=15.6,7.2 \mathrm{~Hz}, 1 \mathrm{H}), 6.22(\mathrm{~d}, J=1.2 \mathrm{~Hz}, 1 \mathrm{H}), 5.87(\mathrm{dd}, J=15.6,1.2 \mathrm{~Hz}, 1 \mathrm{H}), 4.15(\mathrm{q}, J=7.2 \mathrm{~Hz}, 2 \mathrm{H})$, $3.67(\mathrm{t}, J=7.6 \mathrm{~Hz}, 1 \mathrm{H}), 2.89-2.77(\mathrm{~m}, 2 \mathrm{H}), 2.40(\mathrm{~m}, 2 \mathrm{H}), 2.16(\mathrm{~s}, 3 \mathrm{H}), 2.12(\mathrm{t}, J=7.6 \mathrm{~Hz}, 2 \mathrm{H}), 1.25(\mathrm{t}, J=7.6 \mathrm{~Hz}$, 
$3 \mathrm{H})$

${ }^{13}$ C NMR (100 MHz, CDCl $) \delta$ 199.5, 178.7, 166.3, 146.8, 139.4, 133.4, 133.2, 132.4, 128.3, 127.7, 127.6, 126.2, $126.0,126.0,125.7,122.8,118.2,108.2,60.3,51.1,35.6,35.2,24.5,19.7,14.2$

IR (neat) $v 3055,2986,2305,1714,1654,1602,1421,1265,1210,896,746 \mathrm{~cm}^{-1}$

HRMS (ESI) m/z: $[\mathrm{M}+\mathrm{Na}]^{+}$Calcd for $\mathrm{C}_{25} \mathrm{H}_{26} \mathrm{O}_{4} \mathrm{Na}^{+}$413.1723; Found 413.1721

$[\alpha]_{\mathrm{D}}^{29}+27.6\left(c 1.14, \mathrm{CHCl}_{3}\right)$

The enantiomeric ratio was determined by HPLC using CHIRALPACK ${ }^{\circledR}$ IC ( $n$-Hexane: $i$-PrOH $=49: 1$; flow rate $1.0 \mathrm{ml} / \mathrm{min}$, major isomer $t_{\mathrm{R}}=103.3 \mathrm{~min}$, minor isomer $\left.\mathrm{t}_{\mathrm{R}}=89.8 \mathrm{~min}\right)(>99 \% e e)$.

$\mathbf{R}_{\mathbf{f}}(n$-Hexane: EtOAc $=3: 1$, color reagent: $p$-anisaldehyde): 0.36

Ethyl (R,E)-5-(3-acetyl-4-hydroxycyclohexa-1,3-dien-1-yl)-5-(furan-2-yl)pent-2-enoate (7j)

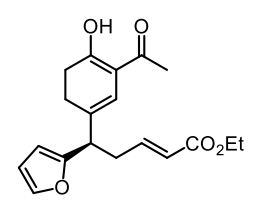

Yield: $80 \%(79.9 \mathrm{mg})$

Physical State: yellow oil

${ }^{1}$ H NMR (400 MHz, $\left.\mathbf{C D C l}_{3}\right) \delta 7.32(\mathrm{~d}, J=1.6 \mathrm{~Hz}, 1 \mathrm{H}), 6.88(\mathrm{dt}, J=15.6,7.2 \mathrm{~Hz}, 1 \mathrm{H}), 6.30(\mathrm{dd}, J=3.2,2.0 \mathrm{~Hz}$, $1 \mathrm{H}), 6.09-6.07(\mathrm{~m}, 2 \mathrm{H}), 5.86(\mathrm{~d}, J=15.6 \mathrm{~Hz}, 1 \mathrm{H}), 4.16(\mathrm{q}, J=7.2 \mathrm{~Hz}, 2 \mathrm{H}), 3.64$ (t, $J=7.6 \mathrm{~Hz}, 1 \mathrm{H}), 2.77$ (dtd, $J=$ 15.2, 7.2, $1.2 \mathrm{~Hz}, 1 \mathrm{H}), 2.62(\mathrm{dtd}, J=15.2,7.2,1.2 \mathrm{~Hz}, 1 \mathrm{H}), 2.45(\mathrm{t}, J=7.6 \mathrm{~Hz}, 2 \mathrm{H}), 2.19(\mathrm{t}, J=8.4 \mathrm{~Hz}, 2 \mathrm{H}), 2.10$ (s, 3H), $1.26(\mathrm{t}, J=7.2 \mathrm{~Hz}, 3 \mathrm{H})$

${ }^{13}$ C NMR (100 MHz, CDCl $) \delta$ 199.4, 178.8, 166.2, 155.3, 145.9, 141.6, 130.6, 123.0, 119.6, 110.1, 108.0, 105.9, $60.3,45.3,35.1,33.9,23.3,19.6,14.2$

IR (neat) $v 2981,1717,1655,1609,1368,1269,1207,1039 \mathrm{~cm}^{-1}$

HRMS (ESI) m/z: [M+Na] ${ }^{+}$Calcd for $\mathrm{C}_{19} \mathrm{H}_{22} \mathrm{O}_{5} \mathrm{Na}^{+}$353.1359; Found 353.1363

$[\alpha]_{\mathrm{D}}^{27}-38.3\left(c 1.10, \mathrm{CHCl}_{3}\right)$

The enantiomeric ratio was determined by HPLC using CHIRALPACK ${ }^{\circledR} \mathrm{IF}(n$-Hexane: $i$-PrOH $=249: 1$; flow rate $1.0 \mathrm{ml} / \mathrm{min}$, major isomer $\mathrm{t}_{\mathrm{R}}=38.9 \mathrm{~min}$, minor isomer $\left.\mathrm{t}_{\mathrm{R}}=35.3 \mathrm{~min}\right)(96 \%$ ee $)$.

$\mathbf{R}_{\mathbf{f}}(n$-Hexane: EtOAc $=3: 1$, color reagent: $p$-anisaldehyde): 0.35

Diethyl (R,E)-5-(3-acetyl-4-hydroxycyclohexa-1,3-dien-1-yl)hex-2-enedioate (7k)<smiles>CCOCCCC(OCC)c1ccc(O)c(C(C)=O)c1</smiles>

Yield: $54 \%$ (54.2 mg)

Physical State: yellow oil

${ }^{1} \mathbf{H}$ NMR (400 MHz, $\left.\mathbf{C D C l}_{3}\right) \delta 6.83(\mathrm{dt}, J=15.6,7.6 \mathrm{~Hz}, 1 \mathrm{H}), 6.12(\mathrm{~s}, 1 \mathrm{H}), 5.86(\mathrm{~d}, J=15.6 \mathrm{~Hz}, 1 \mathrm{H}), 4.19-4.12$ 
(m, 4H), $3.23(\mathrm{t}, J=7.6 \mathrm{~Hz}, 1 \mathrm{H}), 2.74(\mathrm{dtd}, J=14.8,7.6,1.2 \mathrm{~Hz}, 1 \mathrm{H}), 2.54-2.46(\mathrm{~m}, 3 \mathrm{H}), 2.33-2.29(\mathrm{~m}, 2 \mathrm{H}), 2.10$ (s, 3H), $1.26(\mathrm{t}, J=7.2 \mathrm{~Hz}, 3 \mathrm{H}), 1.24(\mathrm{t}, J=7.2 \mathrm{~Hz}, 3 \mathrm{H})$

${ }^{13}$ C NMR (100 MHz, $\left.\mathbf{C D C l}_{3}\right) \delta$ 199.2, 179.4, 172.3, 166.1, 145.1, 127.4, 123.3, 121.2, 108.0, 61.0, 60.3, 51.4, 34.9, $32.7,23.7,19.6,14.2,14.2$

IR (neat) $v 3056,2986,1723,1655,1611,1421,1369,1265,1185,1038,897,744 \mathrm{~cm}^{-1}$

HRMS (ESI) m/z: $[\mathrm{M}+\mathrm{Na}]^{+}$Calcd for $\mathrm{C}_{18} \mathrm{H}_{24} \mathrm{O}_{6} \mathrm{Na}^{+}$359.1465; Found 359.1454

$[\alpha]_{\mathrm{D}}^{28}-23.4\left(c 1.04, \mathrm{CHCl}_{3}\right)$

The enantiomeric ratio was determined by HPLC using CHIRALPACK ${ }^{\circledR}$ IF $(n$-Hexane: $i$-PrOH $=49: 1$; flow rate $1.0 \mathrm{ml} / \mathrm{min}$, major isomer $\mathrm{t}_{\mathrm{R}}=35.2 \mathrm{~min}$, minor isomer $\left.\mathrm{t}_{\mathrm{R}}=30.9 \mathrm{~min}\right)(89 \%$ ee $)$.

$\mathbf{R}_{\mathbf{f}}(n$-Hexane: EtOAc $=3: 1$, color reagent: $p$-anisaldehyde): 0.23

Ethyl (R,E)-5-(3-acetyl-4-hydroxycyclohexa-1,3-dien-1-yl)-5-(dimethyl(phenyl)silyl)pent-2-enoate (7l)<smiles>CCOC=CCC1CCC(O)C(CC)C1</smiles>

Yield: $48 \%(65.8 \mathrm{mg})$

Physical State: yellow oil

${ }^{1}$ H NMR (400 MHz, $\left.\mathbf{C D C l}_{3}\right) \delta$ 7.49-7.47 (m, 2H), 7.38-7.35 (m, 3H), $6.83(\mathrm{dt}, J=15.6,7.2 \mathrm{~Hz}, 1 \mathrm{H}), 5.74(\mathrm{dt}, J=$ 15.6, $1.2 \mathrm{~Hz}, 1 \mathrm{H}), 5.68(\mathrm{~s}, 1 \mathrm{H}), 4.14(\mathrm{q}, J=7.2 \mathrm{~Hz}, 2 \mathrm{H}), 2.42-2.38(\mathrm{~m}, 2 \mathrm{H}), 2.32-2.26(\mathrm{~m}, 2 \mathrm{H}), 2.04-2.01(\mathrm{~m}, 1 \mathrm{H})$, $2.00(\mathrm{~s}, 3 \mathrm{H}), 1.97-1.91(\mathrm{~m}, 1 \mathrm{H}), 1.25(\mathrm{t}, J=7.2 \mathrm{~Hz}, 3 \mathrm{H}), 0.36(\mathrm{~s}, 3 \mathrm{H}), 0.33(\mathrm{~s}, 3 \mathrm{H})$

${ }^{13}$ C NMR (100 MHz, CDCl $) \delta$ 199.4, 177.2, 166.5, 148.9, 136.9, 133.9, 133.9, 131.6, 129.4, 127.8, 127.8, 121.6, $116.9,108.6,60.2,36.9,35.0,31.1,27.0,14.2,-4.1,-4.5$

IR (neat) $v 2957,1717,1650,1607,1427,1403,1368,1304,1266,1207,1180,1114,1047,863,833,815,738$, $703 \mathrm{~cm}^{-1}$

HRMS (ESI) m/z: $[\mathrm{M}+\mathrm{Na}]^{+}$Calcd for $\mathrm{C}_{23} \mathrm{H}_{30} \mathrm{O}_{4} \mathrm{SiNa}^{+}$421.1806; Found 421.1804 $[\alpha]_{\mathrm{D}}^{23}+2.80\left(c 1.06, \mathrm{CHCl}_{3}\right)$

The enantiomeric ratio was determined by HPLC using CHIRALPACK ${ }^{\circledR} \mathrm{IC}(n$-Hexane: $i$-PrOH $=49: 1$; flow rate $1.0 \mathrm{ml} / \mathrm{min}$, major isomer $t_{\mathrm{R}}=31.3 \mathrm{~min}$, minor isomer $\left.\mathrm{t}_{\mathrm{R}}=24.1 \mathrm{~min}\right)(>99 \%$ ee $)$

$\mathbf{R}_{\mathbf{f}}(n$-Hexane: EtOAc $=3: 1$, color reagent: $p$-anisaldehyde $): 0.40$

\section{Ethyl (R,E)-5-(3-benzoyl-4-hydroxycyclohexa-1,3-dien-1-yl)-5-phenylpent-2-enoate (7m)}<smiles>CCOC=CCc1ccccc1CC=CCO</smiles>

Yield: $64 \%(76.9 \mathrm{mg})$

Physical State: yellow oil 
${ }^{1}$ H NMR (400 MHz, $\left.\mathbf{C D C l}_{3}\right) \delta$ 7.60-7.58 (m, 2H), 7.50-7.46 (m, 3H), 7.32-7.28 (m, 2H), 7.24-7.18 (m, 3H), 6.91 $(\mathrm{dt}, J=15.6,6.4 \mathrm{~Hz}, 1 \mathrm{H}), 6.26(\mathrm{~d}, J=0.8 \mathrm{~Hz}, 1 \mathrm{H}), 5.77(\mathrm{dt}, J=15.6,1.6 \mathrm{~Hz}, 1 \mathrm{H}), 4.19(\mathrm{q}, J=7.2 \mathrm{~Hz}, 2 \mathrm{H}), 3.51$ (t, $J=7.6 \mathrm{~Hz}, 1 \mathrm{H}), 2.74-2.58(\mathrm{~m}, 2 \mathrm{H}), 2.47(\mathrm{td}, J=7.6,2.4 \mathrm{~Hz}, 2 \mathrm{H}), 2.26-2.16(\mathrm{~m}, 2 \mathrm{H}), 1.28(\mathrm{t}, J=7.2 \mathrm{~Hz}, 3 \mathrm{H})$

${ }^{13} \mathbf{C}$ NMR (100 MHz, $\left.\mathbf{C D C l}_{3}\right) \delta 203.6,173.7,166.3,146.9,142.3,134.1,133.9,130.8,128.9,128.9,128.6,128.6$, $128.3,128.3,127.6,127.6,126.8,122.7,119.6,107.6,60.3,51.0,36.3,35.5,24.6,14.3$

IR (neat) $v 2981,1716,1654,1604,1563,1383,1273,1207,702 \mathrm{~cm}^{-1}$

HRMS (ESI) m/z: $[\mathrm{M}+\mathrm{Na}]^{+}$Calcd for $\mathrm{C}_{26} \mathrm{H}_{26} \mathrm{O}_{4} \mathrm{Na}^{+}$425.1723; Found 425.1718

$[\alpha]_{\mathrm{D}}^{27}+38.5\left(c 0.960, \mathrm{CHCl}_{3}\right)$

The enantiomeric ratio was determined by HPLC using CHIRALPACK ${ }^{\circledR}$ IF $(n$-Hexane: $i$-PrOH $=49: 1$; flow rate $1.0 \mathrm{ml} / \mathrm{min}$, major isomer $t_{R}=19.7 \mathrm{~min}$, minor isomer $\left.t_{R}=17.2 \mathrm{~min}\right)(94 \%$ ee $)$.

$\mathbf{R}_{\mathbf{f}}(n$-Hexane: EtOAc $=3: 1$, color reagent: $p$-anisaldehyde): 0.40

Ethyl $(R, E)-5-(4-h y d r o x y-3-(p e n t-4-e n o y l) c y c l o h e x a-1,3-d i e n-1-y l)-5-p h e n y l p e n t-2-e n o a t e ~(7 n)$<smiles>C=CC=CCc1cc(O)cc(C(=O)CC=C)c1</smiles>

Yield: $65 \%(74.4 \mathrm{mg})$

Physical State: yellow oil

${ }^{1}$ H NMR (400 MHz, CDCl $) \delta 7.31(\mathrm{t}, J=7.2 \mathrm{~Hz}, 2 \mathrm{H}), 7.24-7.19(\mathrm{~m}, 3 \mathrm{H}), 6.88(\mathrm{dt}, J=15.6,7.2 \mathrm{~Hz}, 1 \mathrm{H}), 6.15$ (s, $1 \mathrm{H}), 5.91-5.81(\mathrm{~m}, 2 \mathrm{H}), 5.09(\mathrm{dt}, J=16.8,1.6 \mathrm{~Hz}, 1 \mathrm{H}), 5.03(\mathrm{dt}, J=10.0,1.6 \mathrm{~Hz}, 1 \mathrm{H}), 4.15(\mathrm{q}, J=7.2 \mathrm{H}, 2 \mathrm{H}), 3.51$ $(\mathrm{t}, J=7.6 \mathrm{~Hz}, 1 \mathrm{H}), 2.81-2.73(\mathrm{~m}, 1 \mathrm{H}), 2.72-2.65(\mathrm{~m}, 1 \mathrm{H}), 2.54-2.50(\mathrm{~m}, 1 \mathrm{H}), 2.42-2.38(\mathrm{~m}, 4 \mathrm{H}), 2.12(\mathrm{t}, J=3.6 \mathrm{~Hz}$, $2 \mathrm{H}), 1.26(\mathrm{t}, J=7.2 \mathrm{~Hz}, 3 \mathrm{H})$

${ }^{13}$ C NMR (100 MHz, CDCl $) \delta$ 199.3, 181.3, 166.3, 146.8, 142.1, 136.8, 133.3, 128.6, 128.6, 127.6, 127.6, 126.8, $122.8,117.7,115.6,107.7,60.2,51.0,35.7,35.1,32.2,29.9,24.5,14.2$;

IR (neat) $v 2981,2934,1718,1653,1600,1450,1393,1368,1270,1205,1156,1039,702 \mathrm{~cm}^{-1}$

HRMS (ESI) m/z: $[\mathrm{M}+\mathrm{Na}]^{+}$Calcd for $\mathrm{C}_{24} \mathrm{H}_{28} \mathrm{O}_{4} \mathrm{Na}^{+}$403.1880; Found 403.1888

$[\alpha]_{\mathrm{D}}^{27}+26.7\left(c 0.840, \mathrm{CHCl}_{3}\right)$

The enantiomeric ratio was determined by HPLC using CHIRALPACK ${ }^{\circledR}$ IB $(n$-Hexane: $i$-PrOH $=99: 1$; flow rate $1.0 \mathrm{ml} / \mathrm{min}$, major isomer $t_{\mathrm{R}}=13.0 \mathrm{~min}$, minor isomer $\left.t_{\mathrm{R}}=11.4 \mathrm{~min}\right)(>99 \% e e)$.

$\mathbf{R}_{\mathbf{f}}(n$-Hexane: EtOAc $=3: 1$, color reagent: $p$-anisaldehyde $): 0.48$

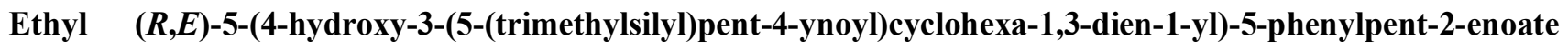
(7o)

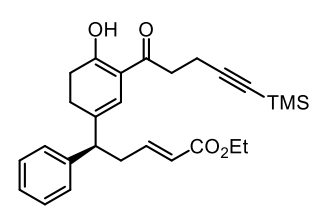


Yield: $66 \%(93.4 \mathrm{mg})$

Physical State: yellow oil

${ }^{1}$ H NMR (400 MHz, $\left.\mathbf{C D C l}_{3}\right) \delta 7.31(\mathrm{t}, J=7.2 \mathrm{~Hz}, 2 \mathrm{H}), 7.25-7.23(\mathrm{~m}, 1 \mathrm{H}), 7.20-7.18(\mathrm{~m}, 2 \mathrm{H}), 6.87$ (dt, $J=15.6$, $7.2 \mathrm{~Hz}, 1 \mathrm{H}), 6.20(\mathrm{~s}, 1 \mathrm{H}), 5.82(\mathrm{~d}, J=15.6 \mathrm{~Hz}, 1 \mathrm{H}), 4.16(\mathrm{q}, J=7.2 \mathrm{~Hz}, 2 \mathrm{H}), 3.51(\mathrm{t}, J=7.6 \mathrm{~Hz}, 1 \mathrm{H}), 2.80-2.67$ (m, $4 \mathrm{H}), 2.59-2.56(\mathrm{~m}, 2 \mathrm{H}), 2.40(\mathrm{t}, J=7.6 \mathrm{~Hz}, 2 \mathrm{H}), 2.11(\mathrm{t}, J=7.6 \mathrm{~Hz}, 2 \mathrm{H}) 1.27(\mathrm{t}, J=7.2 \mathrm{~Hz}, 3 \mathrm{H}), 0.16(\mathrm{~s}, 9 \mathrm{H})$

${ }^{13} \mathbf{C}$ NMR (100 MHz, $\left.\mathbf{C D C l}_{3}\right) \delta 198.9,179.7,166.3,146.7,141.9,133.9,128.6,128.6,127.6,127.6,126.8$, $122.8,117.3,108.0,105.3,85.6,60.3,51.1,35.7,34.9,32.0,31.6,22.6,16.3,14.2,0.06,0.06,0.06$

IR (neat) $v 2959,2177,1719,1653,1600,1315,1268,1250,1205,1156,1043,844,760,701 \mathrm{~cm}^{-1}$

HRMS (ESI) m/z: $[\mathrm{M}+\mathrm{Na}]^{+} \mathrm{Calcd}$ for $\mathrm{C}_{27} \mathrm{H}_{34} \mathrm{O}_{4} \mathrm{SiNa}^{+} 473.2119$; Found 473.2122

$[\alpha]_{\mathrm{D}}^{27}+23.0\left(c 1.13, \mathrm{CHCl}_{3}\right)$

The enantiomeric ratio was determined by HPLC using CHIRALPACK ${ }^{\circledR}$ IA ( $n$-Hexane: $i$-PrOH $=199: 1$; flow rate $1.0 \mathrm{ml} / \mathrm{min}$, major isomer $\mathrm{t}_{\mathrm{R}}=6.90 \mathrm{~min}$, minor isomer $\left.\mathrm{t}_{\mathrm{R}}=6.09 \mathrm{~min}\right)(>99 \% e e)$.

$\mathbf{R}_{\mathbf{f}}(n$-Hexane: $\mathrm{EtOAc}=3: 1$, color reagent: $p$-anisaldehyde): 0.52

Ethyl (R,E)-5-(3-acetyl-4-hydroxycyclohepta-1,3-dien-1-yl)-5-phenylpent-2-enoate (7p)

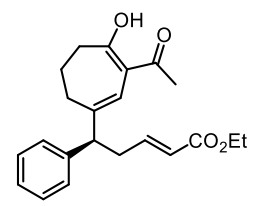

Yield: $37 \%(41.5 \mathrm{mg})$

Physical State: yellow oil

${ }^{1}$ H NMR (400 MHz, CDCl $)$ ) $\delta$ 7.33-7.30 (m, 2H), 7.25-7.21 (m, 3H), $6.92(\mathrm{dt}, J=15.6,7.2 \mathrm{~Hz}, 1 \mathrm{H}), 6.08(\mathrm{~s}, 1 \mathrm{H})$, $5.85(\mathrm{~d}, J=15.9 \mathrm{~Hz}, 1 \mathrm{H}), 4.16(\mathrm{q}, J=7.2 \mathrm{~Hz}, 2 \mathrm{H}), 3.58$ (t, $J=7.2 \mathrm{~Hz}, 1 \mathrm{H}), 2.83-2.70(\mathrm{~m}, 2 \mathrm{H}), 2.29$ (t, $J=7.2 \mathrm{~Hz}$, 2H), 2.13 (s, 3H), 2.06-1.94 (m, 2H), 1.79-1.70 (m, 2H), 1.26 (t, $J=7.2 \mathrm{~Hz}, 3 \mathrm{H})$

${ }^{13}$ C NMR (100 MHz, $\left.\mathbf{C D C l}_{3}\right) \delta$ 195.0, 190.7, 166.3, 147.0, 142.4, 142.3, 128.5, 128.5, 127.5, 127.5, 126.8, 122.8, $120.4,110.0,60.2,52.7,36.1,35.3,30.4,28.1,23.3,14.2$

IR (neat) $\vee 2937,1718,1654,1601,1448,1367,1264,1185,1156,702 \mathrm{~cm}^{-1}$

HRMS (ESI) m/z: $[\mathrm{M}+\mathrm{Na}]^{+}$Calcd for $\mathrm{C}_{22} \mathrm{H}_{26} \mathrm{O}_{4} \mathrm{Na}^{+}$377.1724; Found 377.1727

$[\alpha]_{\mathrm{D}}{ }^{27}-4.00\left(c 1.06, \mathrm{CHCl}_{3}\right)$

The enantiomeric ratio was determined by HPLC using CHIRALPACK ${ }^{\circledR} \mathrm{IC}(n$-Hexane: $i$-PrOH $=99: 1$; flow rate $1.0 \mathrm{ml} / \mathrm{min}$, major isomer $\mathrm{t}_{\mathrm{R}}=21.4 \mathrm{~min}$, minor isomer $\left.\mathrm{t}_{\mathrm{R}}=17.6 \mathrm{~min}\right)(92 \% e e)$.

$\mathbf{R}_{\mathbf{f}}(n$-Hexane: $\mathrm{EtOAc}=3: 1$, color reagent: $p$-anisaldehyde): 0.48 


\subsection{Procedure of $1 \mathrm{mmol}$ scale}
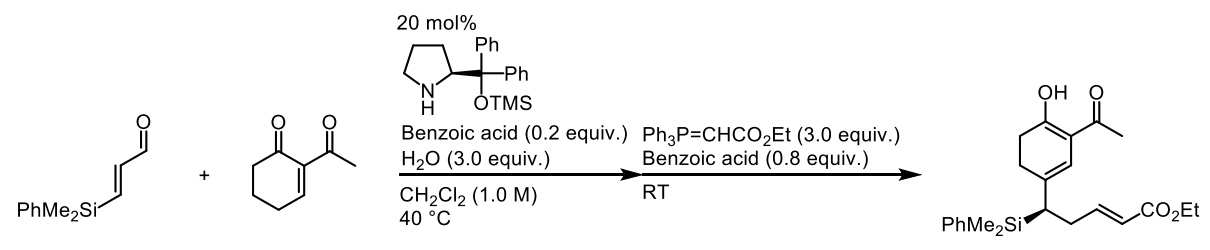

71

To a solution of ketone $(0.21 \mathrm{~g}, 1.5 \mathrm{mmol})$ and aldehyde $(0.19 \mathrm{~g}, 1.0 \mathrm{mmol})$ in $\mathrm{CH}_{2} \mathrm{Cl}_{2}(1.0 \mathrm{~mL}), \mathrm{H}_{2} \mathrm{O}(54 \mu \mathrm{L}, 3.0$ mmol), benzoic acid ( $24 \mathrm{mg}, 0.20 \mathrm{mmol}$ ), and catalyst (65 mg, $0.20 \mathrm{mmol})$ were added at $40{ }^{\circ} \mathrm{C}$. After $2 \mathrm{~h}$, benzoic acid $(98 \mathrm{mg}, 0.80 \mathrm{mmol})$ and Wittig reagent $(1.0 \mathrm{~g}, 3.0 \mathrm{mmol})$ ware added to the reaction mixture. After $24 \mathrm{~h}$, the reaction was quenched by the addition of sat. aq. $\mathrm{NaHCO}_{3}(10 \mathrm{~mL})$. Separated aqueous layer was extracted with $\mathrm{CH}_{2} \mathrm{Cl}_{2}(15 \mathrm{~mL} \times 3)$. The combined organic layers were washed with brine $(30 \mathrm{~mL})$, dried by $\mathrm{Na}_{2} \mathrm{SO}_{4}$, filtered and concentrated in vacuo. The residue was purified by flash chromatography on silica gel $(n$-Hexane: EtOAc $=18: 1)$ to give compound $7 \mathbf{l}(0.18 \mathrm{~g}, 46 \%,>99 \% e e)$.

\subsection{Oxidation of compound 71}

Ethyl (R,E)-5-(3-acetyl-4-hydroxyphenyl)-5-(dimethyl(phenyl)silyl)pent-2-enoate (8)

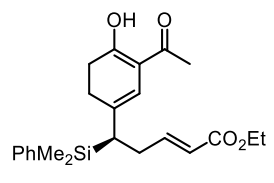

71

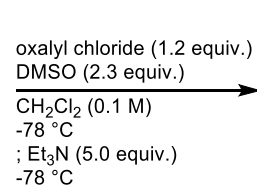

$-78^{\circ} \mathrm{C}$

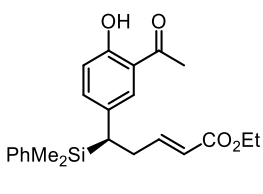

To a mixture of DMSO $(15 \mu \mathrm{L}, 0.21 \mathrm{mmol})$ and $\mathrm{CH}_{2} \mathrm{Cl}_{2}(0.30 \mathrm{~mL})$, oxalyl chloride $(9.0 \mu \mathrm{L}, 0.11 \mathrm{mmol})$ in $\mathrm{CH}_{2} \mathrm{Cl}_{2}(0.30 \mathrm{~mL})$ was added at $-78^{\circ} \mathrm{C}$ under Ar atmosphere. After $15 \mathrm{~min}$, to a stirred solution, a solution of diene $7 \mathbf{l}(36 \mathrm{mg}, 0.090 \mathrm{mmol})$ in $\mathrm{CH}_{2} \mathrm{Cl}_{2}(0.30 \mathrm{~mL})$ was added at $-78{ }^{\circ} \mathrm{C}$. After $30 \mathrm{~min}$, to a stirred solution, $\mathrm{Et}{ }_{3} \mathrm{~N}(63 \mu \mathrm{L}$, $0.45 \mathrm{mmol}$ ) was added at $-78{ }^{\circ} \mathrm{C}$. After $1 \mathrm{~h}$, the reaction solution was quenched by the addition of sat. aq. $\mathrm{NH}_{4} \mathrm{Cl}$ $(3 \mathrm{~mL})$ at $0{ }^{\circ} \mathrm{C}$. Separated aqueous layer was extracted with $\mathrm{CH}_{2} \mathrm{Cl}_{2}(5 \mathrm{~mL} \times 3)$. The combined organic layers were washed with brine $(10 \mathrm{~mL})$, dried by $\mathrm{Na}_{2} \mathrm{SO}_{4}$, filtered and concentrated in vacuo. The residue was purified by flash chromatography on silica gel $(n$-Hexane: EtOAc $=15: 1)$ to give corresponding phenol $8(22 \mathrm{mg}, 61 \%)$ as a yellow oil.

Yield: $61 \%(22 \mathrm{mg})$

Physical State: yellow oil

${ }^{1}$ H NMR (400 MHz, $\left.\mathbf{C D C l}_{3}\right) \delta 12.0(\mathrm{~s}, 1 \mathrm{H}), 7.40-7.35(\mathrm{~m}, 2 \mathrm{H}), 7.34-7.33(\mathrm{~m}, 3 \mathrm{H}), 7.04(\mathrm{dd}, J=8.8,2.4 \mathrm{~Hz}, 1 \mathrm{H})$, $6.99(\mathrm{~d}, J=2.4 \mathrm{~Hz}, 1 \mathrm{H}), 6.84(\mathrm{~d}, J=8.8 \mathrm{~Hz}, 1 \mathrm{H}), 6.76(\mathrm{dt}, J=15.6,7.2 \mathrm{~Hz}, 1 \mathrm{H}), 5.68(\mathrm{dt}, J=15.6,1.6 \mathrm{~Hz}, 1 \mathrm{H})$, $4.09(\mathrm{q}, J=7.2 \mathrm{~Hz}, 2 \mathrm{H}), 2.68-2.52(\mathrm{~m}, 2 \mathrm{H}), 2.36(\mathrm{~s}, 3 \mathrm{H}), 2.35-2.32(\mathrm{~m}, 1 \mathrm{H}), 1.22(\mathrm{t}, J=7.2 \mathrm{~Hz}, 3 \mathrm{H}), 0.26(\mathrm{~s}, 3 \mathrm{H})$, $0.26(\mathrm{~s}, 3 \mathrm{H})$

${ }^{13}$ C NMR (100 MHz, $\left.\mathbf{C D C l}_{3}\right) \delta 204.4,166.3,159.8,148.2,136.2,135.9,134.1,134.1131 .4,129.5,129.4,127.8$, $127.8,122.1,119.3,118.1,60.1,34.7,32.1,26.4,14.2,-4.6,-5.0$ 
IR (neat) $v 2958,1716,1643,1482,1428,1368,1251,1203,1049,814,737,701 \mathrm{~cm}^{-1}$

HRMS (ESI) m/z: $[\mathrm{M}+\mathrm{Na}]^{+}$Calcd for $\mathrm{C}_{23} \mathrm{H}_{28} \mathrm{O}_{4} \mathrm{SiNa}^{+} 419.1650$; Found 419.1648

$[\alpha]_{\mathrm{D}}^{26}-19.8\left(c 0.930, \mathrm{CHCl}_{3}\right)$

$\mathbf{R}_{\mathbf{f}}(n$-Hexane: EtOAc $=3: 1$, color reagent: $p$-anisaldehyde): 0.40

\subsection{The determination of the absolute configuration of 9}

\subsubsection{TBS protection and oxidation}

Ethyl (R,E)-5-(3-acetyl-4-((tert-butyldimethylsilyl)oxy)phenyl)-5-(dimethyl(phenyl)silyl)pent-2-enoate (S10)

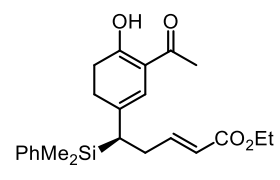

71

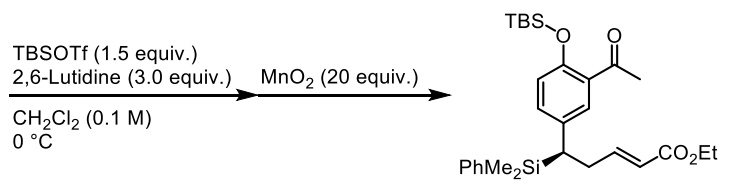

S10

To a solution of diene 71 (180 mg, $0.46 \mathrm{mmol})$ in $\mathrm{CH}_{2} \mathrm{Cl}_{2}(23 \mathrm{~mL})$, 2,6-lutidine (160 $\left.\mu \mathrm{L}, 1.4 \mathrm{mmol}\right)$ and TBSOTf $(160 \mu \mathrm{L}, 0.69 \mathrm{mmol})$ were added at $0{ }^{\circ} \mathrm{C}$ under Ar atmosphere. After $10 \mathrm{~min}, \mathrm{MnO}_{2}(800 \mathrm{mg}, 9.2 \mathrm{mmol})$ was added. After $10 \mathrm{~min}$, the reaction mixture was Celite ${ }^{\mathcal{O}}$ filtered and concentareted in vacuo. The residue was purified by flash chromatography on silica gel $(n$-Hexane: EtOAc $=15: 1)$ to give compound $\mathbf{S 1 0}(97 \mathrm{mg}, 42 \%)$ as a colorless oil.

Yield: $42 \%$ (97.3 mg)

Physical State: colorless oil

${ }^{1}$ H NMR (400 MHz, $\left.\mathbf{C D C l}_{3}\right) \delta$ 7.37-7.31 (m, 5H), $7.15(\mathrm{~d}, J=2.4 \mathrm{~Hz}, 1 \mathrm{H}), 6.82(\mathrm{dd}, J=8.4,2.4 \mathrm{~Hz}, 1 \mathrm{H})$, 6.77-6.69 (m, 2H), $5.66(\mathrm{~d}, J=15.6 \mathrm{~Hz}, 1 \mathrm{H}), 4.15-4.05(\mathrm{~m}, 3 \mathrm{H}), 2.59-2.54(\mathrm{~m}, 4 \mathrm{H}), 2.34(\mathrm{dd}, J=10.8,4.8 \mathrm{~Hz}, 1 \mathrm{H})$, $1.21(\mathrm{t}, J=7.2 \mathrm{~Hz}, 3 \mathrm{H}), 0.99(\mathrm{~s}, 9 \mathrm{H}), 0.26(\mathrm{~s}, 3 \mathrm{H}), 0.24(\mathrm{~s}, 6 \mathrm{H}), 0.20(\mathrm{~s}, 3 \mathrm{H})$

${ }^{13} \mathbf{C}$ NMR (100 MHz, $\left.\mathbf{C D C l}_{3}\right) \delta 199.4,177.2,166.5,148.9,136.9,133.9,133.9,133.0,131.6,129.4,127.9,127.8$, $127.8,121.6,116.9,108.6,60.0,34.6,32.1,31.6,31.3,25.9,22.6,18.4,14.2,-4.11,-4.11,-4.5,-4.5$

IR (neat) $\vee 2956,2931,1719,1683,1489,1255,915,840,815,782 \mathrm{~cm}^{-1}$

HRMS (ESI) m/z: [M+Na] ${ }^{+}$Calcd for $\mathrm{C}_{29} \mathrm{H}_{42} \mathrm{O}_{4} \mathrm{Si}_{2} \mathrm{Na}^{+}$533.2514; Found 533.2513

$[\alpha]_{\mathrm{D}}^{26}-21.6\left(c 0.930, \mathrm{CHCl}_{3}\right)$

$\mathbf{R}_{\mathbf{f}}(n$-Hexane: EtOAc $=3: 1$, color reagent: Hanessian's stain reagent): 0.60

\subsubsection{Tamao-Fleming oxidation}

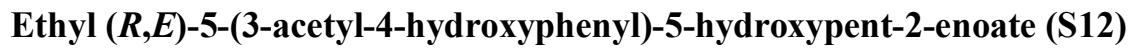

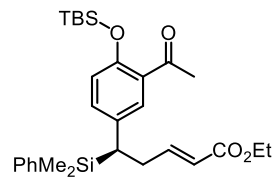

s10

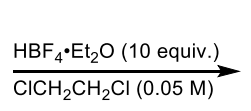

$$
\mathrm{FMe}_{2}
$$<smiles>CCO/C=C\C[C@H](C)OCC</smiles>

S11

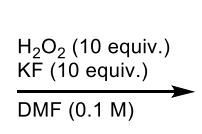

S25

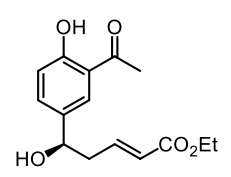

S12 
To a solution of compound $\mathbf{S 1 0}(23 \mathrm{mg}, 0.045 \mathrm{mmol})$ in $\mathrm{ClCH}_{2} \mathrm{CH}_{2} \mathrm{Cl}(0.90 \mathrm{~mL}), \mathrm{HBF}_{4} \cdot \mathrm{Et}_{2} \mathrm{O}(63 \mu \mathrm{L}, 0.45$ mmol) was added at $60{ }^{\circ} \mathrm{C}$. After $3 \mathrm{~h}$, the reaction was quenched by the addition sat. aq. $\mathrm{NaHCO}_{3}(3 \mathrm{~mL})$ at $0{ }^{\circ} \mathrm{C}$. Separated aqueous layer was extracted with $\mathrm{CH}_{2} \mathrm{Cl}_{2}(5 \mathrm{~mL} \times 3)$. The combined organic layers were washed with brine $(10 \mathrm{~mL})$, dried by $\mathrm{Na}_{2} \mathrm{SO}_{4}$, filtered and concentrated in vacuo to give corresponding compound $\mathbf{S 1 1}$. This crude material was used without further purification.

To a solution of crude in DMF $(0.45 \mathrm{~mL}), \mathrm{KF}(26 \mathrm{mg}, 0.45 \mathrm{mmol})$ and $30 \%$ aq. $\mathrm{H}_{2} \mathrm{O}_{2}(46 \mu \mathrm{L}, 0.45 \mathrm{mmol})$ were added at $40{ }^{\circ} \mathrm{C}$. After $1.5 \mathrm{~h}$, the reaction was quenched by the addition of dimethyl sulfide $(1 \mathrm{~mL})$ at $0{ }^{\circ} \mathrm{C}$, poured water $(3 \mathrm{~mL})$ and extracted with diethyl ether. $(3 \mathrm{~mL} \times 3)$ The combined organic layers were washed with water $(5$ $\mathrm{mL}$ ) and brine $(5 \mathrm{~mL})$, dried by $\mathrm{MgSO}_{4}$, filtered and concentrated in vacuo. The residue was purified by flash chromatography on silica gel ( $n$-Hexane: EtOAc $=6: 1)$ to give alcohol $\mathbf{S 1 2}(3.3 \mathrm{mg}, 26 \%)$ as an orange oil.

Yield: $26 \%(3.3 \mathrm{mg})$

Physical State: orange oil

${ }^{1}$ H NMR (400 MHz, CDCl $) \delta 12.2(\mathrm{~s}, 1 \mathrm{H}), 7.72(\mathrm{~d}, J=2.4 \mathrm{~Hz}, 1 \mathrm{H}), 7.45(\mathrm{dd}, J=8.4,2.4 \mathrm{~Hz}, 1 \mathrm{H}), 6.98-6.91(\mathrm{~m}$, $2 \mathrm{H}), 5.90(\mathrm{~d}, J=16.0 \mathrm{~Hz}, 1 \mathrm{H}), 4.82(\mathrm{dd}, J=7.6,5.6 \mathrm{~Hz}, 1 \mathrm{H}), 4.18(\mathrm{q}, J=7.2 \mathrm{~Hz}, 2 \mathrm{H}), 2.66-2.61(\mathrm{~m}, 5 \mathrm{H}), 1.28(\mathrm{t}, J$ $=7.2 \mathrm{~Hz}, 3 \mathrm{H})$

${ }^{13} \mathbf{C}$ NMR (100 MHz, $\left.\mathbf{C D C l}_{3}\right) \delta$ 204.4, 166.2, 162.0, 144.0, 134.0, 133.9, 127.9, 124.5, 119.4, 118.7, 72.3, 60.4, $41.9,26.7,14.2$

IR (neat) $v 2964,2931,2874,1645,1469,1378,910,739 \mathrm{~cm}^{-1}$

HRMS (ESI) m/z: $[\mathrm{M}+\mathrm{Na}]^{+} \mathrm{Calcd}$ for $\mathrm{C}_{15} \mathrm{H}_{18} \mathrm{O}_{5} \mathrm{Na}^{+}$301.1047; Found 301.1050

$[\alpha]_{\mathrm{D}}{ }^{25}+26.8\left(c 0.950, \mathrm{CHCl}_{3}\right)$

$\mathbf{R}_{\mathbf{f}}(n$-Hexane: $\mathrm{EtOAc}=2: 1$, color reagent: Hanessian's stain reagent): 0.37

\subsubsection{Methyl esterification}

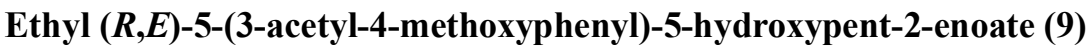
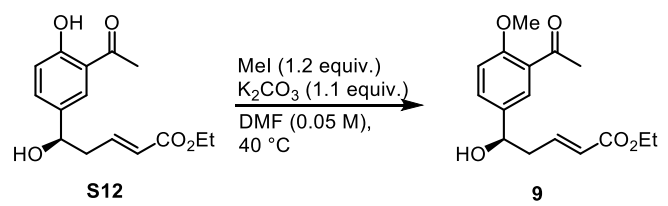

To a solution of alcohol $\mathbf{S 1 2}(4.2 \mathrm{mg}, 15 \mu \mathrm{mol})$ in DMF $(0.30 \mathrm{~mL}), \mathrm{K}_{2} \mathrm{CO}_{3}(2.3 \mathrm{mg}, 17 \mu \mathrm{mol})$ and $\mathrm{MeI}(1.1 \mu \mathrm{L}, 18$ $\mu \mathrm{mol})$ were added at $40{ }^{\circ} \mathrm{C}$. After $2.5 \mathrm{~h}$, the reaction was quenched by the addition of sat. aq. $\mathrm{NH}_{4} \mathrm{Cl}(1 \mathrm{~mL})$. Separated aqueous layer was extracted with diethyl ether $(3 \mathrm{~mL} \times 3)$. The combined organic layers were washed with water $(5 \mathrm{~mL})$ and brine $(5 \mathrm{~mL})$, dried by $\mathrm{MgSO}_{4}$, filtered and concentrated in vacuo. The residue was purified by thin layer chromatography ( $n$-Hexane: EtOAc $=3: 1)$ to give methylether $9(2.7 \mathrm{mg}, 64 \%)$ as a yellow oil.

Yield: $64 \%(2.7 \mathrm{mg})$ 
Physical State: yellow oil

${ }^{1}$ H NMR (400 MHz, CDCl$) \delta 7.71(\mathrm{~d}, J=2.4 \mathrm{~Hz}, 1 \mathrm{H}), 7.49(\mathrm{dd}, J=8.4,2.4 \mathrm{~Hz}, 1 \mathrm{H}), 6.97(\mathrm{~d}, J=8.4 \mathrm{~Hz}, 1 \mathrm{H})$, $6.93(\mathrm{dt}, J=15.6,7.6 \mathrm{~Hz}, 1 \mathrm{H}), 5.90(\mathrm{~d}, J=15.6 \mathrm{~Hz}, 1 \mathrm{H}), 4.82(\mathrm{dd}, J=7.6,5.6 \mathrm{~Hz}, 1 \mathrm{H}), 4.18(\mathrm{q}, J=7.2 \mathrm{~Hz}, 2 \mathrm{H})$, $3.92(\mathrm{~s}, 3 \mathrm{H}), 2.66-2.62(\mathrm{~m}, 2 \mathrm{H}), 2.62(\mathrm{~s}, 3 \mathrm{H}), 1.28(\mathrm{t}, J=7.2 \mathrm{~Hz}, 3 \mathrm{H})$

${ }^{13}$ C NMR (100 MHz, $\left.\mathbf{C D C l}_{3}\right) \delta$ 199.7, 166.2, 158.6, 144.3, 135.6, 131.1, 128.0, 127.8, 124.2, 111.9, 72.3, 60.3, $55.7,41.7,31.8,14.2$

IR (neat) $v 3446,2979,2931,1715,1670,1656,1607,1578,1497,1465,1418,1367,1254,1180,1041,822 \mathrm{~cm}^{-1}$ HRMS (ESI) m/z: $[\mathrm{M}+\mathrm{Na}]^{+}$Calcd for $\mathrm{C}_{16} \mathrm{H}_{20} \mathrm{O}_{5} \mathrm{Na}^{+}$315.1203; Found 315.1200

$[\alpha]_{\mathrm{D}}^{26}+22.9\left(c 0.520, \mathrm{CHCl}_{3}\right)$

$\mathbf{R}_{\mathbf{f}}(n$-Hexane: EtOAc $=2: 1$, color reagent: Hanessian's stain reagent $): 0.33$

\subsubsection{Typical procedure of the synthesis Mosher ester}
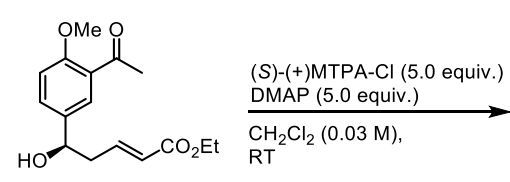

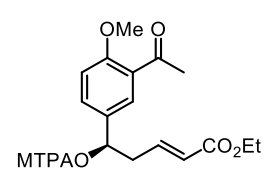

S13

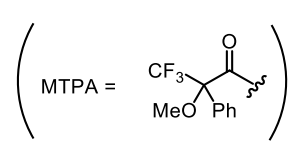

To a solution of alcohol 9 (5.4 mg, $18 \mu \mathrm{mol})$ in $\mathrm{CH}_{2} \mathrm{Cl}_{2}(0.36 \mathrm{~mL})$, 4-dimethylaminopyridine (11 $\left.\mathrm{mg}, 92 \mu \mathrm{mol}\right)$ and $(S)$-(+)- $\alpha$-methoxy- $\alpha$-(trifluoromethyl)phenylacetyl chloride $(17 \mathrm{~mL}, 92 \mu \mathrm{mol})$ were added at room temperature under Ar atmosphere. After $15 \mathrm{~min}$, the reaction was quenched with sat. aq. $\mathrm{NaHCO}_{3}(1 \mathrm{~mL})$ at $0{ }^{\circ} \mathrm{C}$. Separated aqueous layer was extracted with $\mathrm{CH}_{2} \mathrm{Cl}_{2}(3 \mathrm{~mL} \times 3)$. The combined organic layers were washed with brine $(5 \mathrm{~mL})$, dried by $\mathrm{Na}_{2} \mathrm{SO}_{4}$, filtrated and concentrated in vacuo. The residue was purified by flash chromatography on silica gel $(n$-Hexane: EtOAc $=10: 1)$ to give Mosher ester S13 (4.3 mg, 46\%) as a yellow oil.

\section{Ethyl(R,E)-5-(3-acetyl-4-methoxyphenyl)-5-(((R)-3,3,3-trifluoro-2-methoxy-2-phenylpropanoyl)oxy)pent-}

\section{2-enoate (S13)}

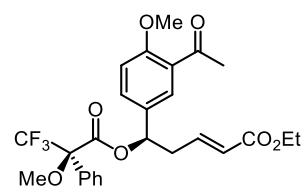

Yield: $46 \%(4.3 \mathrm{mg})$

Physical State: yellow oil

${ }^{1}$ H NMR (400 MHz, $\left.\mathbf{C D C l}_{3}\right) \delta 7.76(\mathrm{~d}, J=2.4 \mathrm{~Hz}, 1 \mathrm{H}), 7.47(\mathrm{dd}, J=8.4,2.4 \mathrm{~Hz}, 1 \mathrm{H}), 7.42-7.40(\mathrm{t}, J=8.4 \mathrm{~Hz}$, $2 \mathrm{H}), 7.37-7.32(\mathrm{~m}, 2 \mathrm{H}), 6.96(\mathrm{~d}, J=8.4 \mathrm{~Hz}, 1 \mathrm{H}), 6.71(\mathrm{dt}, J=15.6,7.2 \mathrm{~Hz}, 1 \mathrm{H}), 6.03(\mathrm{dd}, J=8.4,5.6 \mathrm{~Hz}, 1 \mathrm{H})$, $5.77(\mathrm{~d}, J=15.6 \mathrm{~Hz}, 1 \mathrm{H}), 4.16(\mathrm{q}, J=7.2 \mathrm{~Hz}, 2 \mathrm{H}), 3.93(\mathrm{~s}, 3 \mathrm{H}), 3.42(\mathrm{~s}, 3 \mathrm{H}), 2.83(\mathrm{dt}, J=15.2,7.2 \mathrm{~Hz}, 1 \mathrm{H})$, 2.71-2.64 (m, 1H), $2.61(\mathrm{~s}, 3 \mathrm{H}), 1.27(\mathrm{t}, J=7.2 \mathrm{~Hz}, 3 \mathrm{H})$

${ }^{13}$ C NMR (100 MHz, $\mathbf{C D C l}_{3}$ ) $\delta 199.0,165.8,165.7,159.1,141.7,132.4,132.0,130.2,129.6,128.8,128.4,128.4$, $128.3,127.3,127.3,125.0,125.0,111.9,76.0,60.3,55.7,55.5,38.4,31.8,14.2$

IR (neat) $v 2981,2951,2847,1748,1719,1678,1609,1499,1259,1171,1020,822,720 \mathrm{~cm}^{-1}$

HRMS (ESI) m/z: $[\mathrm{M}+\mathrm{Na}]^{+}$Calcd for $\mathrm{C}_{26} \mathrm{H}_{27} \mathrm{~F}_{3} \mathrm{O}_{7} \mathrm{Na}^{+}$531.1602; Found 531.1605 
$[\alpha]_{\mathrm{D}}^{25}+62.8\left(c 0.360, \mathrm{CHCl}_{3}\right)$

$\mathbf{R}_{\mathbf{f}}(n$-Hexane: EtOAc $=1: 1$, color reagent: Hanessian's stain reagent): 0.70

Ethyl $(R, E)-5-(3-a c e t y l-4-m e t h o x y p h e n y l)-5-((S)-3,3,3-$ trifluoro-2-methoxy-2-phenylpropanoyl)oxy)pent2-enoate (S14)

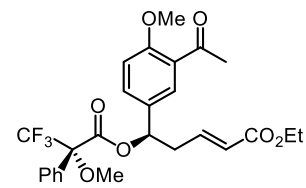

Yield: $32 \%(3.1 \mathrm{mg})$

Physical State: yellow oil

${ }^{1}$ H NMR (400 MHz, $\left.\mathbf{C D C l}_{3}\right) \delta 7.62(\mathrm{~d}, J=2.4 \mathrm{~Hz}, 1 \mathrm{H}), 7.39-7.28(\mathrm{~m}, 5 \mathrm{H}), 6.91(\mathrm{~d}, J=8.8 \mathrm{~Hz}, 1 \mathrm{H}), 6.83(\mathrm{dt}, J=$ 15.6, $7.2 \mathrm{~Hz}, 1 \mathrm{H}), 5.96(\mathrm{dd}, J=8.8,5.2 \mathrm{~Hz}, 1 \mathrm{H}), 5.88(\mathrm{~d}, J=15.6 \mathrm{~Hz}, 1 \mathrm{H}), 4.18$ (q, $J=7.2 \mathrm{~Hz}, 2 \mathrm{H}), 3.92(\mathrm{~s}, 3 \mathrm{H})$, $3.50(\mathrm{~s}, 3 \mathrm{H}), 2.86(\mathrm{dt}, J=15.6,8.0 \mathrm{~Hz}, 1 \mathrm{H}), 2.73-2.66(\mathrm{~m}, 1 \mathrm{H}), 2.59$ (s. $3 \mathrm{H}), 1.27(\mathrm{t}, J=7.2 \mathrm{~Hz}, 3 \mathrm{H})$

${ }^{13}$ C NMR (100 MHz, CDCl $) \delta$ 199.0, 165.8, 165.6, 159.0, 142.1, 132.1, 131.8, 130.3, 129.5, 128.7, 128.3, 128.3, $128.2,127.3,127.3,125.3,125.1,111.8,76.4,60.4,55.7,55.7,38.5,31.7,14.2$

IR (neat) $\vee 2985,2951,2847,1749,1719,1678,1609,1498,1259,1181,1020,821,721 \mathrm{~cm}^{-1}$

HRMS (ESI) m/z: [M+Na] ${ }^{+}$Calcd for $\mathrm{C}_{26} \mathrm{H}_{27} \mathrm{~F}_{3} \mathrm{O}_{7} \mathrm{Na}^{+}$531.1602; Found 531.1600 $[\alpha]_{\mathrm{D}}^{26}+20.8\left(c 0.170, \mathrm{CHCl}_{3}\right)$

$\mathbf{R}_{\mathbf{f}}(n$-Hexane: EtOAc $=1: 1$, color reagent: Hanessian's stain reagent): 0.70

\subsubsection{Results of Mosher method}

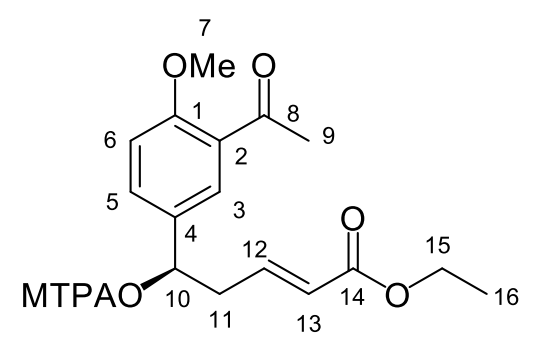

\begin{tabular}{c|c|c|c} 
& $(S)-(+)-$ Mosher ester & $(R)-(-)$-Mosher ester & $\Delta \delta$ \\
\hline 3 & 7.616 & 7.757 & -0.141 \\
6 & 6.907 & 6.963 & -0.056 \\
7 & 3.919 & 3.931 & -0.012 \\
9 & 2.585 & 2.612 & -0.027 \\
11 & $2.863,2.697$ & $2.832,2.681$ & $+0.031,+0.016$ \\
12 & 6.829 & 6.706 & +0.123 \\
13 & 5.880 & 5.771 & +0.109 \\
15 & 4.179 & 4.162 & +0.017 \\
16 & 1.273 & 1.271 & +0.002
\end{tabular}


$510^{\circ} \mathrm{Z}$

$620^{2} \mathrm{Z}$

$960 \mathrm{Z}$
$890^{\circ} \mathrm{Z}$

$810^{\circ} \mathrm{Z}$

901 ' 2

จะะ'乙

$\angle 9 \varepsilon^{\circ} \mathrm{Z}$

ต8ะ 2

b 907 '

sos'z

819'乙-

ĢG'

टह्व

EtG'

699 '

$089 \mathrm{G}$

E69 9

$909 \mathrm{G}$

Gाठ 97

6129

०८Z' 9 -

tot $9-1$

$8 \circ Z^{\circ} 9$

$299^{\circ} L$

$Z \angle 9^{\circ} L>$

$289^{\circ} \mathrm{J}$

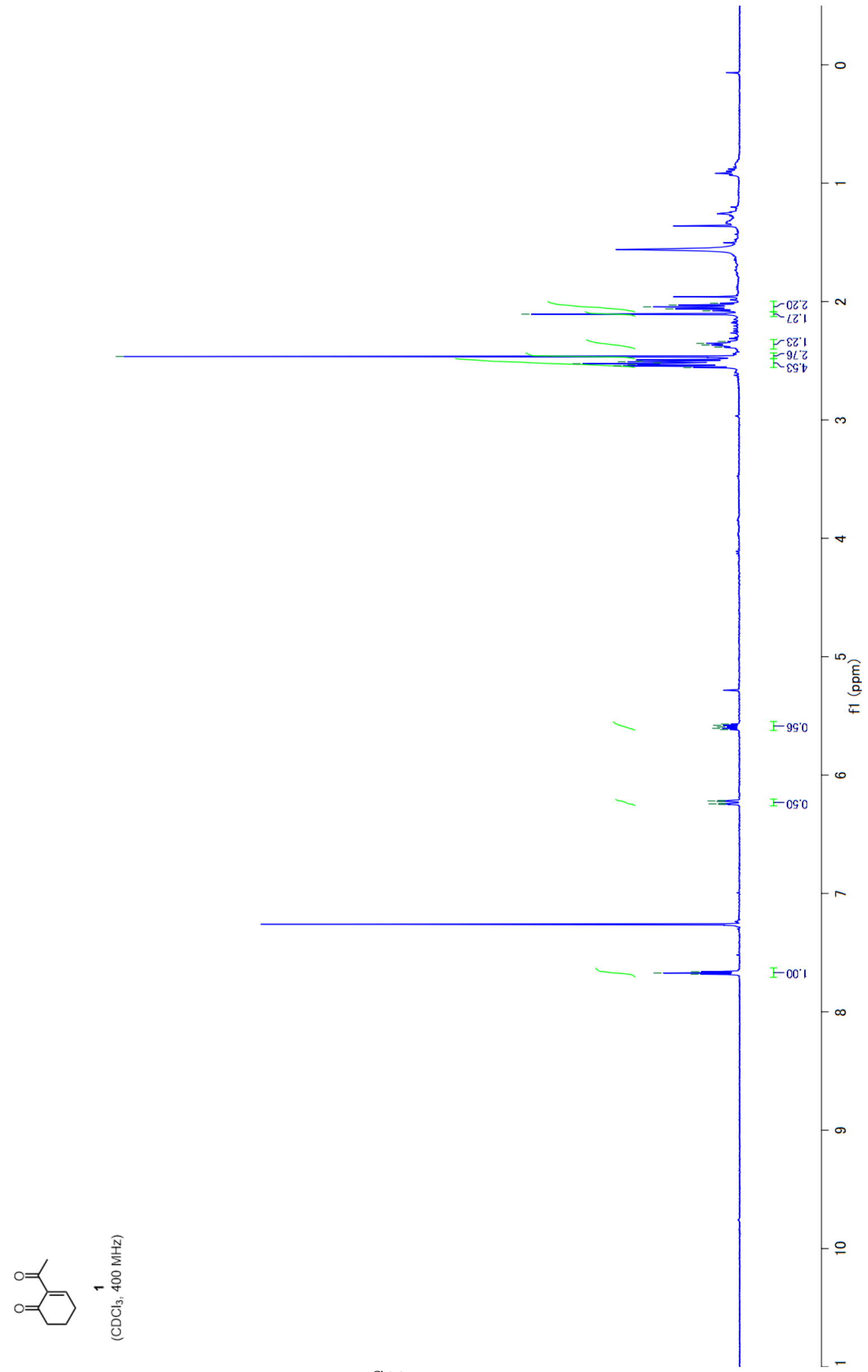



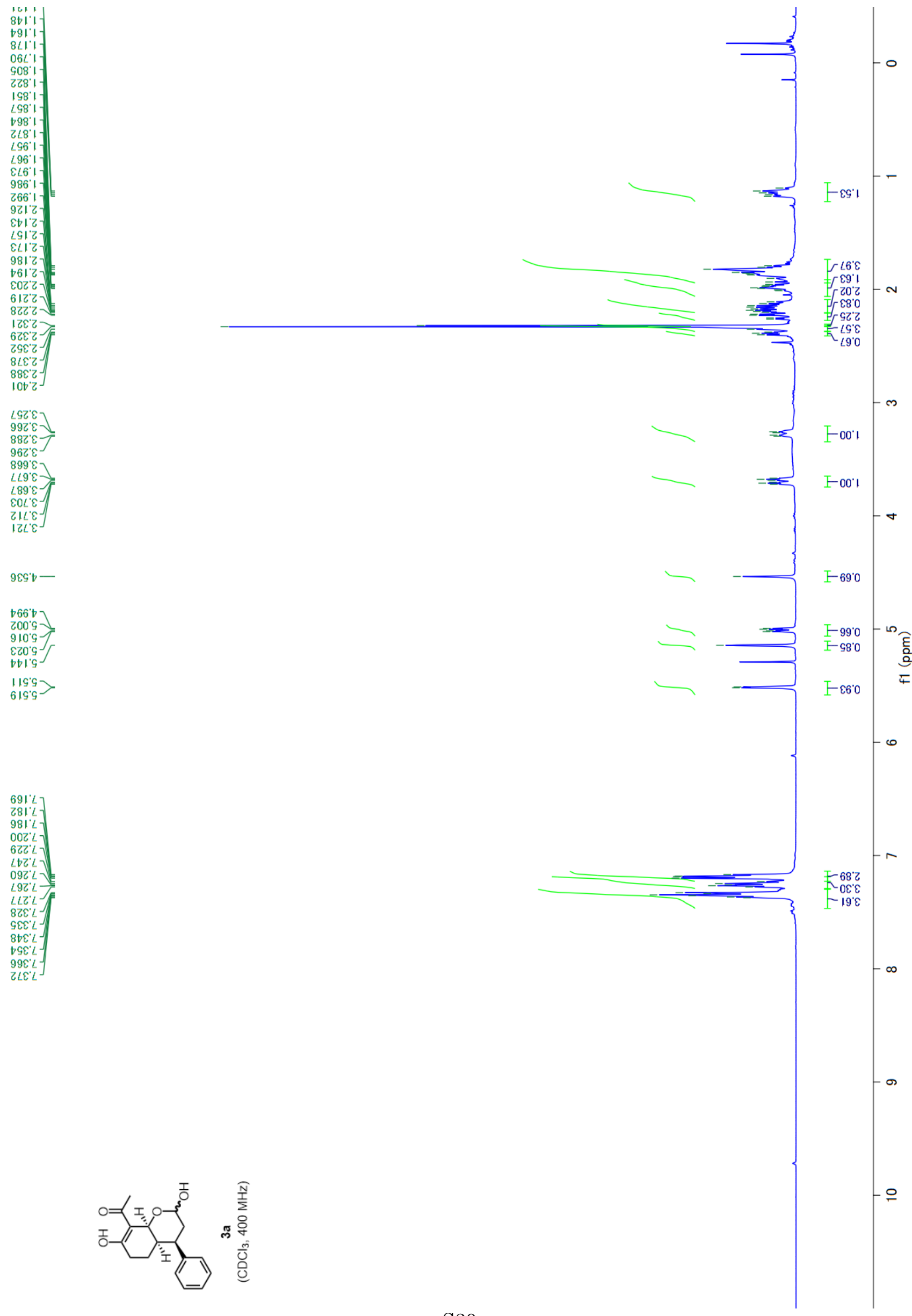
$09 \circ 1-$
$80^{\circ} 91-$

$06^{\circ} \varepsilon \zeta$
$06^{\circ} \varepsilon \zeta$

4 ' 82

Lo. $18>$

LI. $18-$

$01.28-$

966

it $2 \mathrm{~b}$

$t 299$

$81^{\circ} \mathrm{L}$

$86.16-$

เE.96-

+9.801
$16^{\circ} 801$

Zt 9717

เE้ $\measuredangle Z 1-$

与Eเ

$98821-$

$8+161=$
$18201=$

$\sqcup \mathcal{E}^{\circ} 91$
1998

$9600 z-$

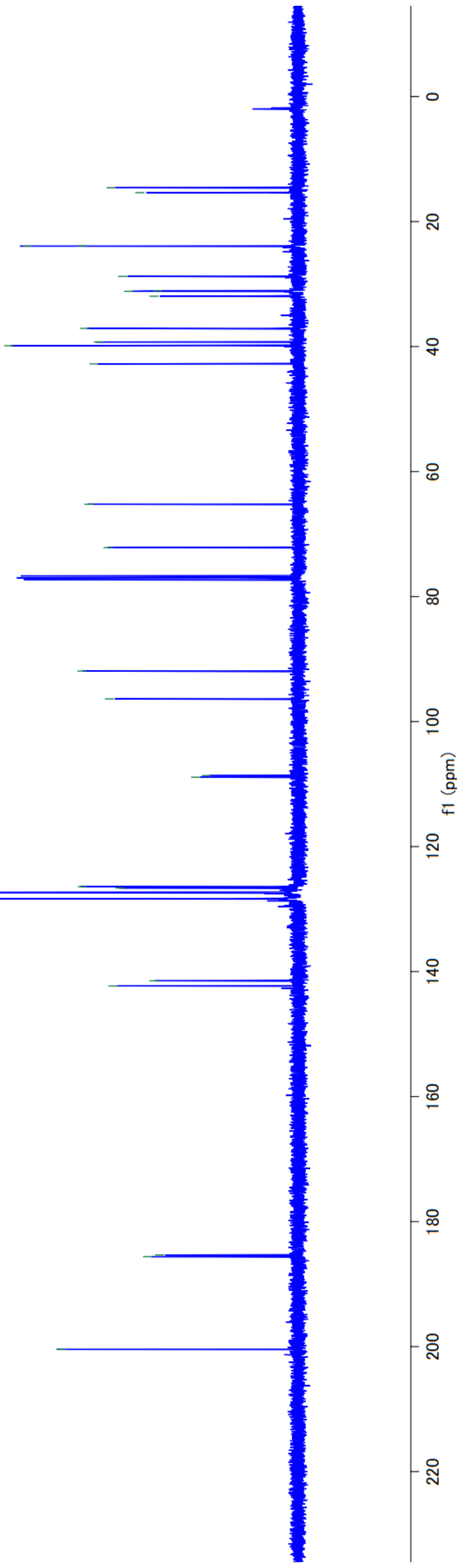




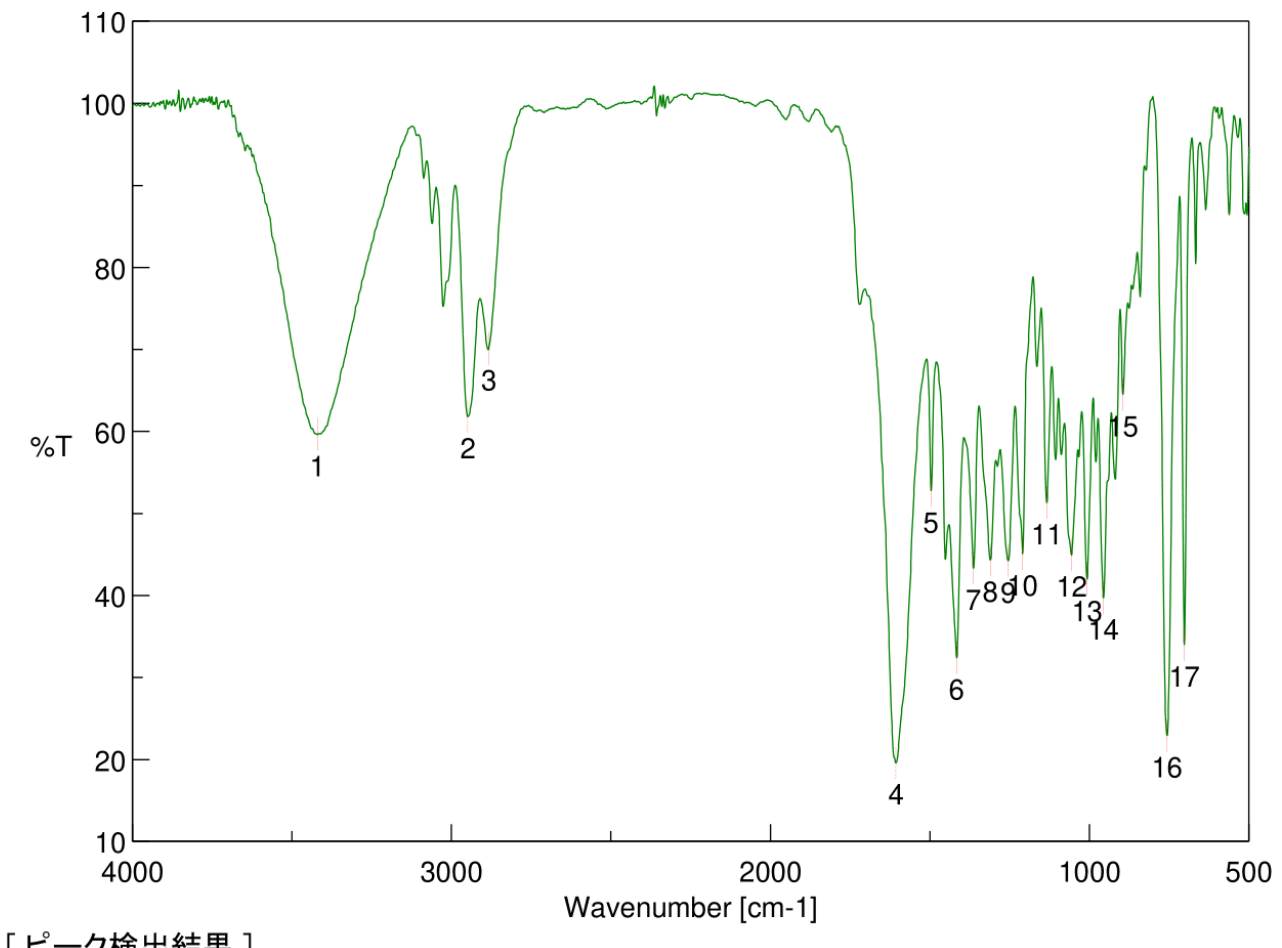

[ピーク検出結果 ]

$\begin{array}{lllllll}\text { No. } & \text { 位置 } & \text { 強度 } & \text { No. } & \text { 位置 } & \text { 強度 } \\ 1 & 3420.14 & 59.6 & 2 & 2948.63 & 61.7786 \\ 3 & 2883.06 & 70.1044 & 4 & 1606.41 & 19.5774 \\ 5 & 1496.49 & 52.7453 & 6 & 1416.46 & 32.3985 \\ 7 & 1363.43 & 43.2959 & 8 & 1310.39 & 44.2738 \\ 9 & 1254.47 & 44.2137 & 10 & 1209.15 & 45.0596 \\ 11 & 1133.94 & 51.2816 & 12 & 1055.84 & 44.9061 \\ 13 & 1007.62 & 41.9625 & 14 & 955.555 & 39.675 \\ 15 & 894.809 & 64.4693 & 16 & 756.923 & 22.8998 \\ 17 & 701.962 & 33.9556 & & & & \end{array}$



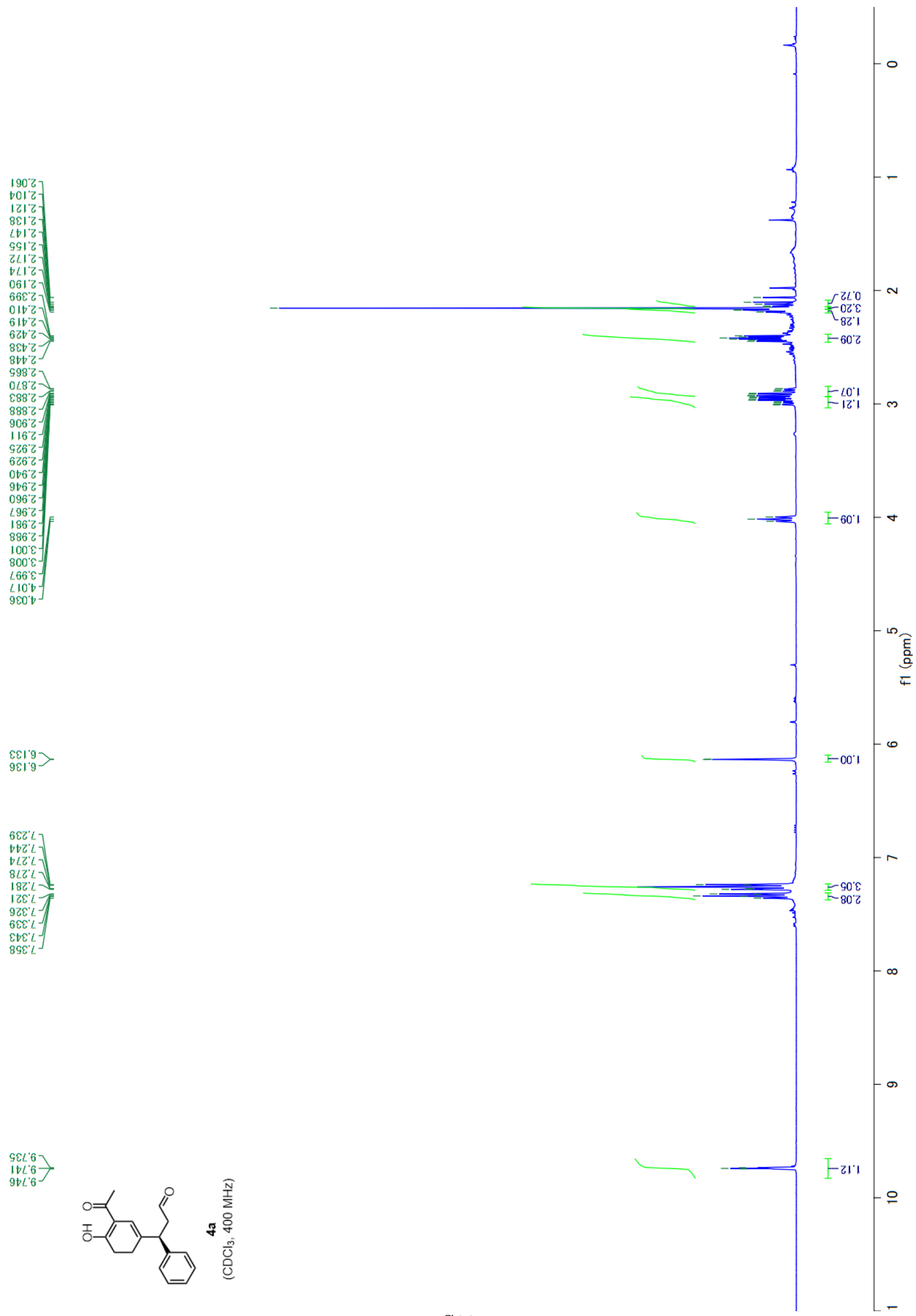

$9 \varepsilon[6]$

$\left.\begin{array}{l}1+L^{\circ} 6 \\ 9 b L^{\circ} 6\end{array}\right\}$

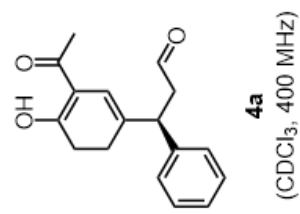


$1961-$

L8 $\mathrm{bz}$

Zo'ต -

$98^{\circ} \mathrm{sb}=$
Z1 20

$56: \angle 01-$

$50811-$

$20^{\circ} \angle Z 1=$
$89^{\circ} \angle Z 1=$

ZI 821

ZLZE। -

$0 t|b|-$

$90621-$

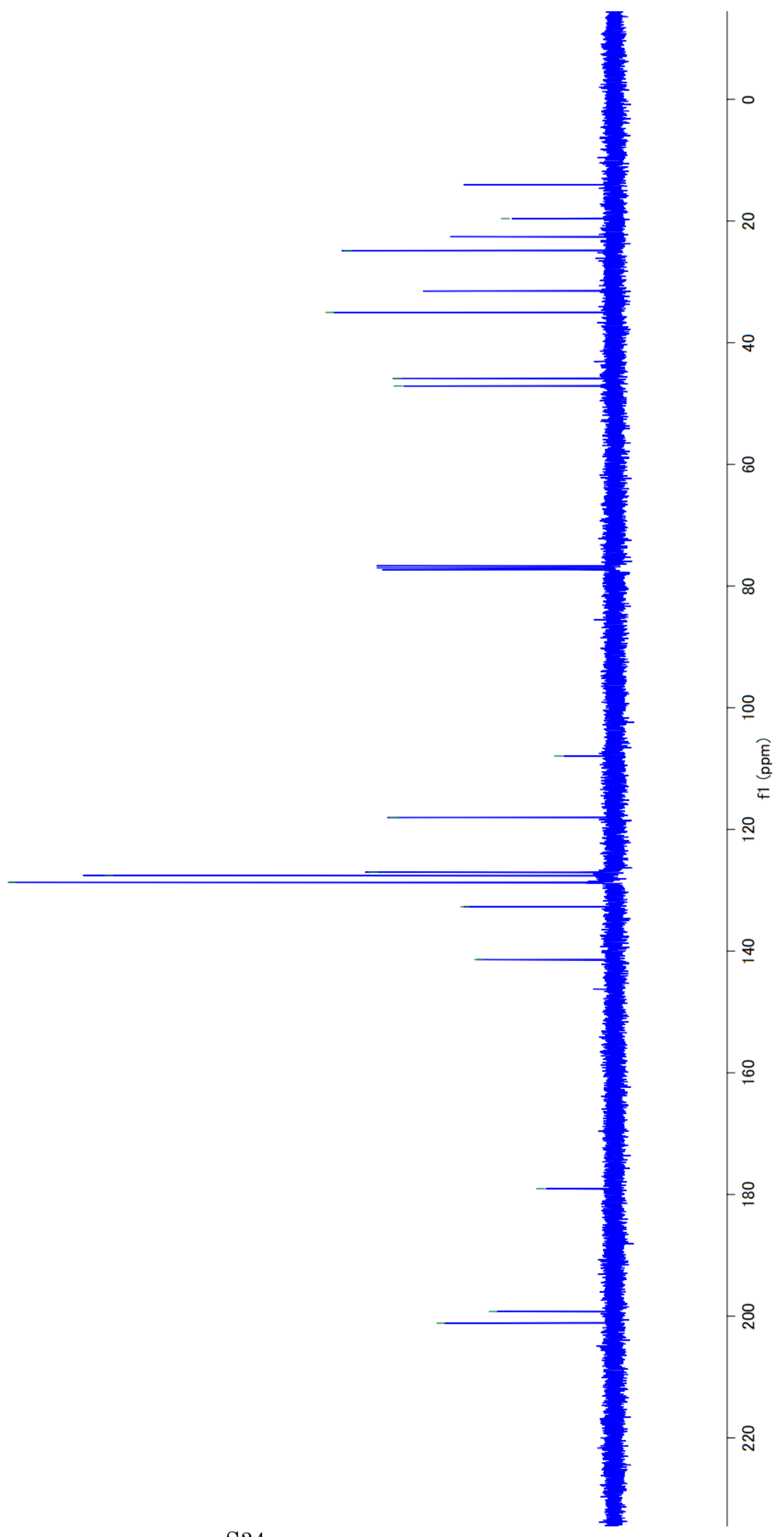

bZ $661=$

$\left\langle<\frac{2}{\frac{1}{1}}\right.$ 


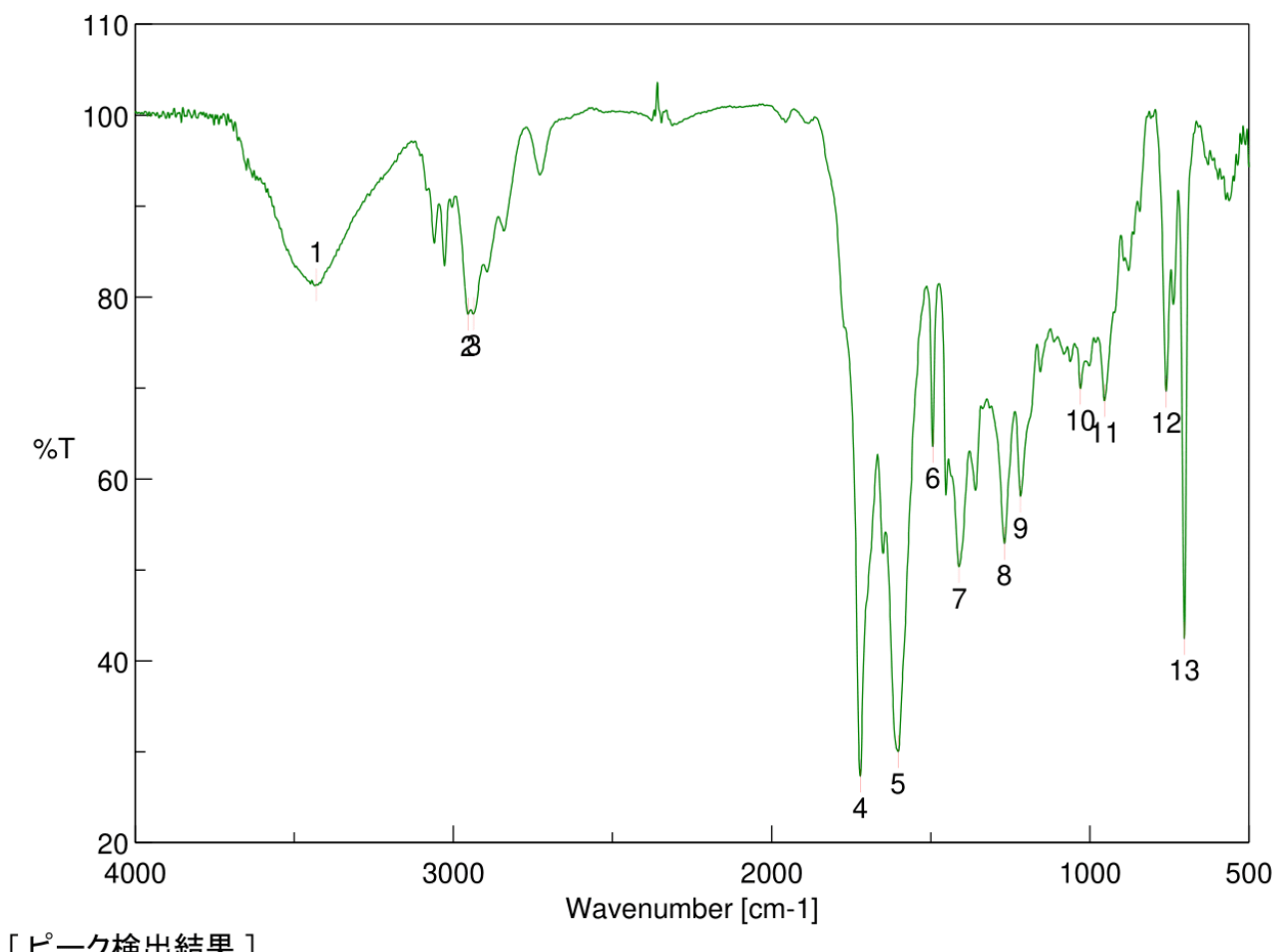

\begin{tabular}{llllll}
\multicolumn{2}{l}{ [ピーク検出結果 ] } & & & & \\
No. & 位置 & 強度 & No. & 位置 & 強度 \\
1 & 3430.74 & 81.3294 & 2 & 2954.41 & 78.0965 \\
3 & 2936.09 & 78.1799 & 4 & 1721.16 & 27.3126 \\
5 & 1602.56 & 29.9853 & 6 & 1493.6 & 63.551 \\
7 & 1410.67 & 50.3391 & 8 & 1267.97 & 52.8666 \\
9 & 1217.83 & 58.108 & 10 & 1029.8 & 69.9251 \\
11 & 953.627 & 68.5847 & 12 & 759.816 & 69.6347 \\
13 & 702.926 & 42.4479 & & &
\end{tabular}

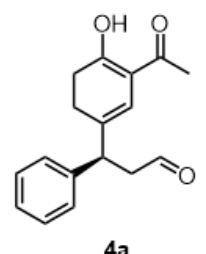




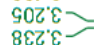

$\varepsilon 飞 9^{\circ} \varepsilon$

$189^{\circ} \frac{1}{2}$

$419^{\circ} \mathrm{C}$

086 't

$986 \circ$

$800^{\circ} \mathrm{s}$

sos's

乙IG

$180^{\circ} L$

$z+00^{\circ} L$

290.2

$9+b^{\circ} L$

$996: 1$

(1) 1

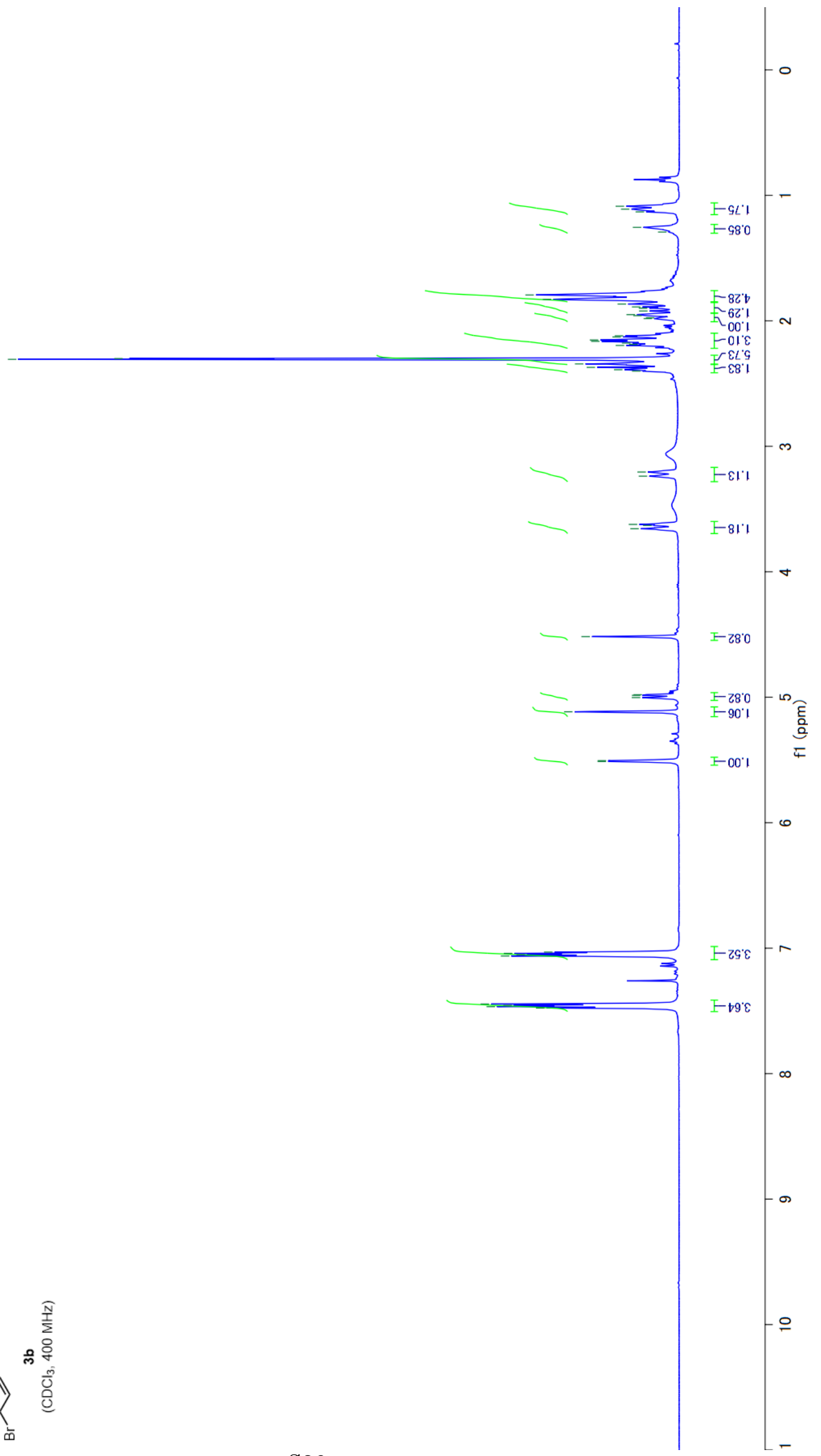




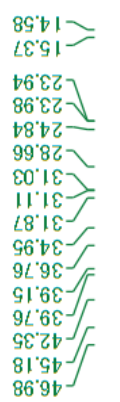

Zวৎ9-

$912 L$

$98.16-$

tว 96 -

LL801-

$\angle Z 0 Z 1$
$Z S^{\circ} 0 Z 1$

2067

$01621]$

ตE⿱ $621-$

Z9. IE।

Z8' IE।

$6 t^{\circ} 0 t 1=$
$9 \varepsilon^{\circ} 1+1=$

98

$00^{\prime} 981-$

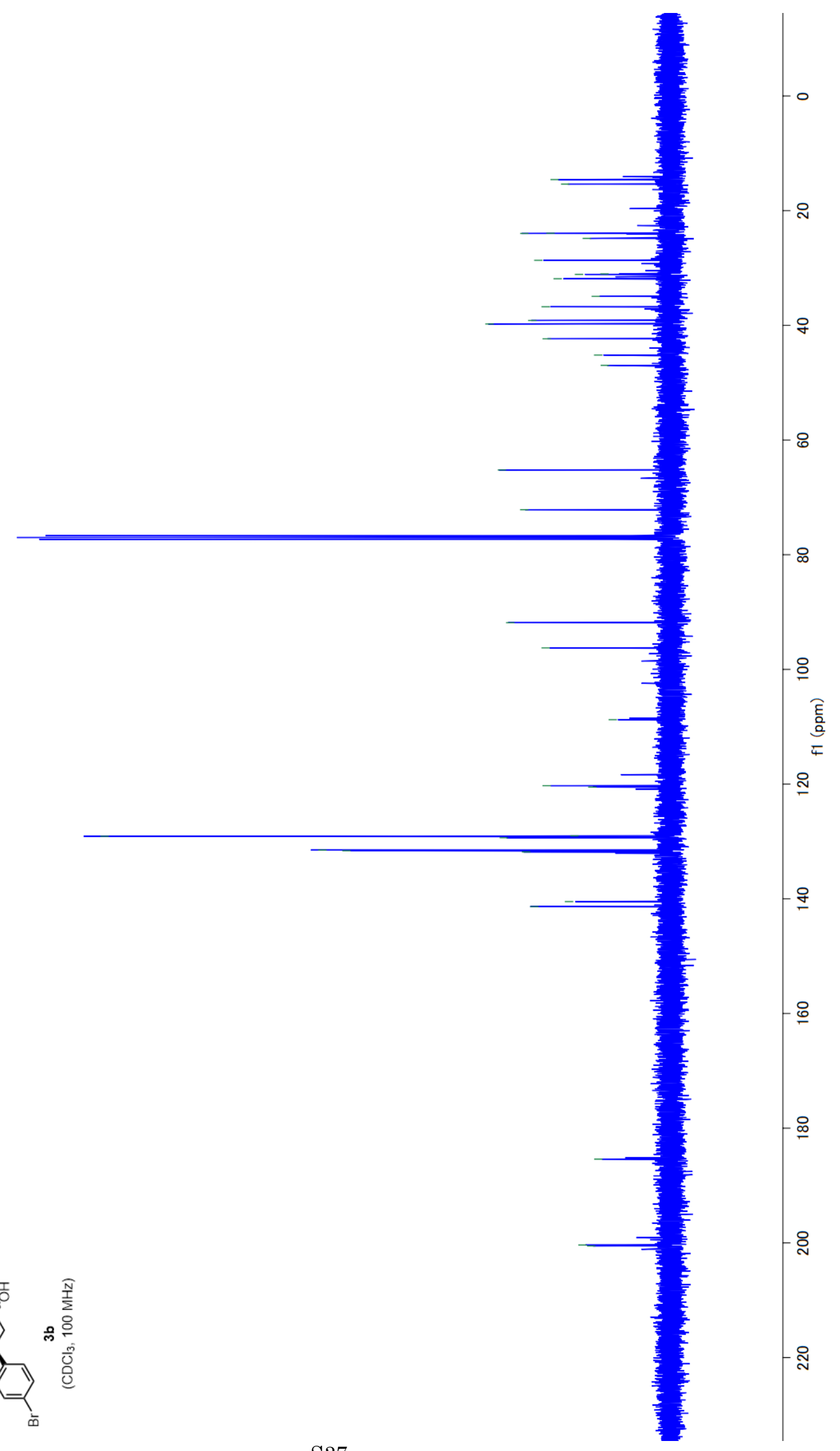




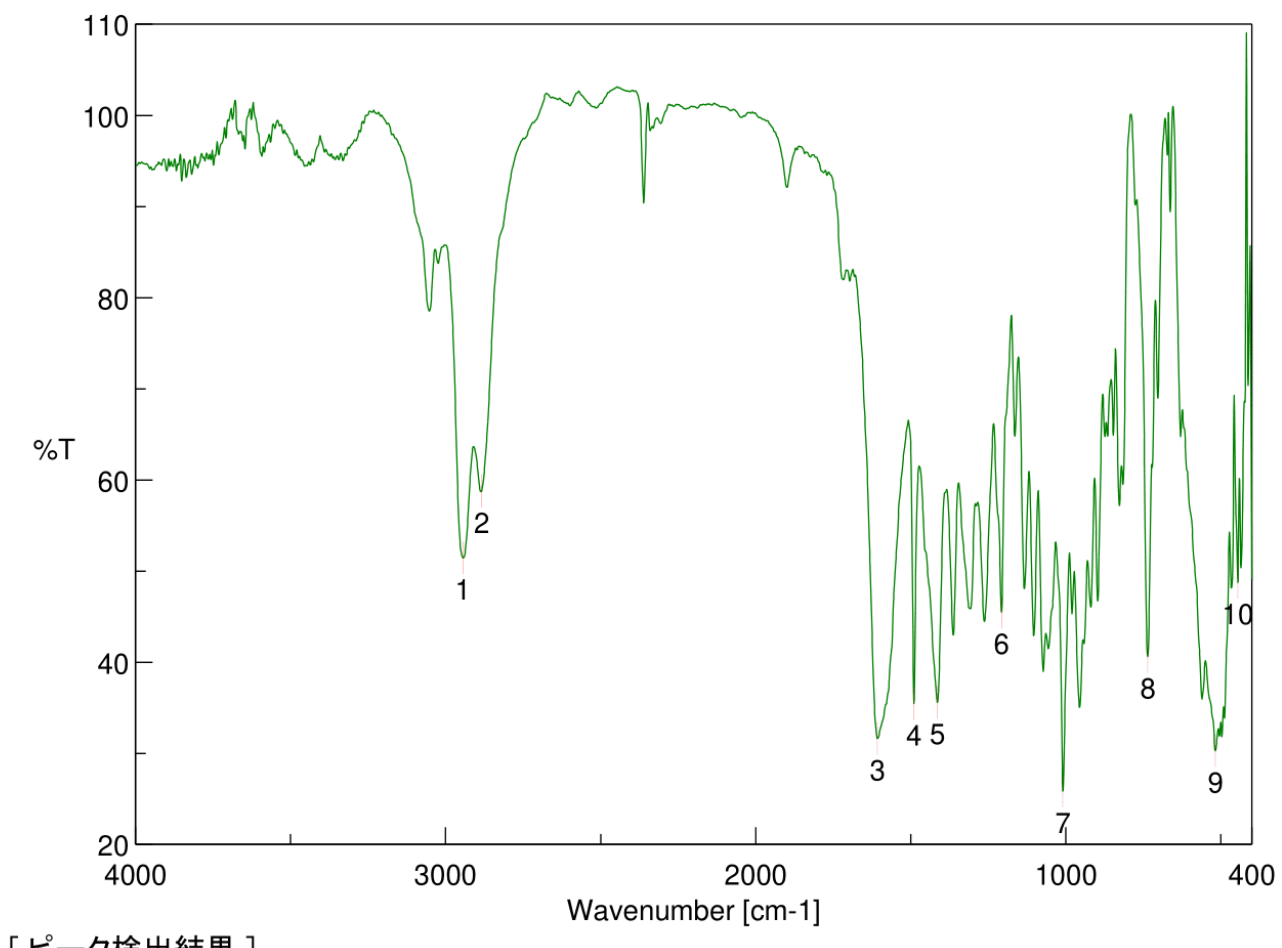

\begin{tabular}{llllll}
\multicolumn{2}{l}{ [ピーク検出結果 ] } & & & & \\
No. & 位置 & 強度 & No. & 位置 & 強度 \\
1 & 2943.8 & 51.4202 & 2 & 2884.02 & 58.7763 \\
3 & 1607.38 & 31.6275 & 4 & 1489.74 & 35.4141 \\
5 & 1413.57 & 35.5728 & 6 & 1207.22 & 45.4593 \\
7 & 1009.55 & 25.8363 & 8 & 735.71 & 40.6156 \\
9 & 517.793 & 30.2493 & 10 & 445.476 & 48.7304
\end{tabular}

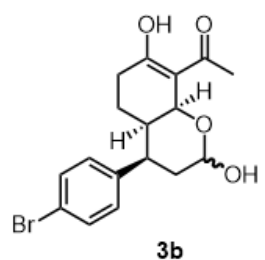




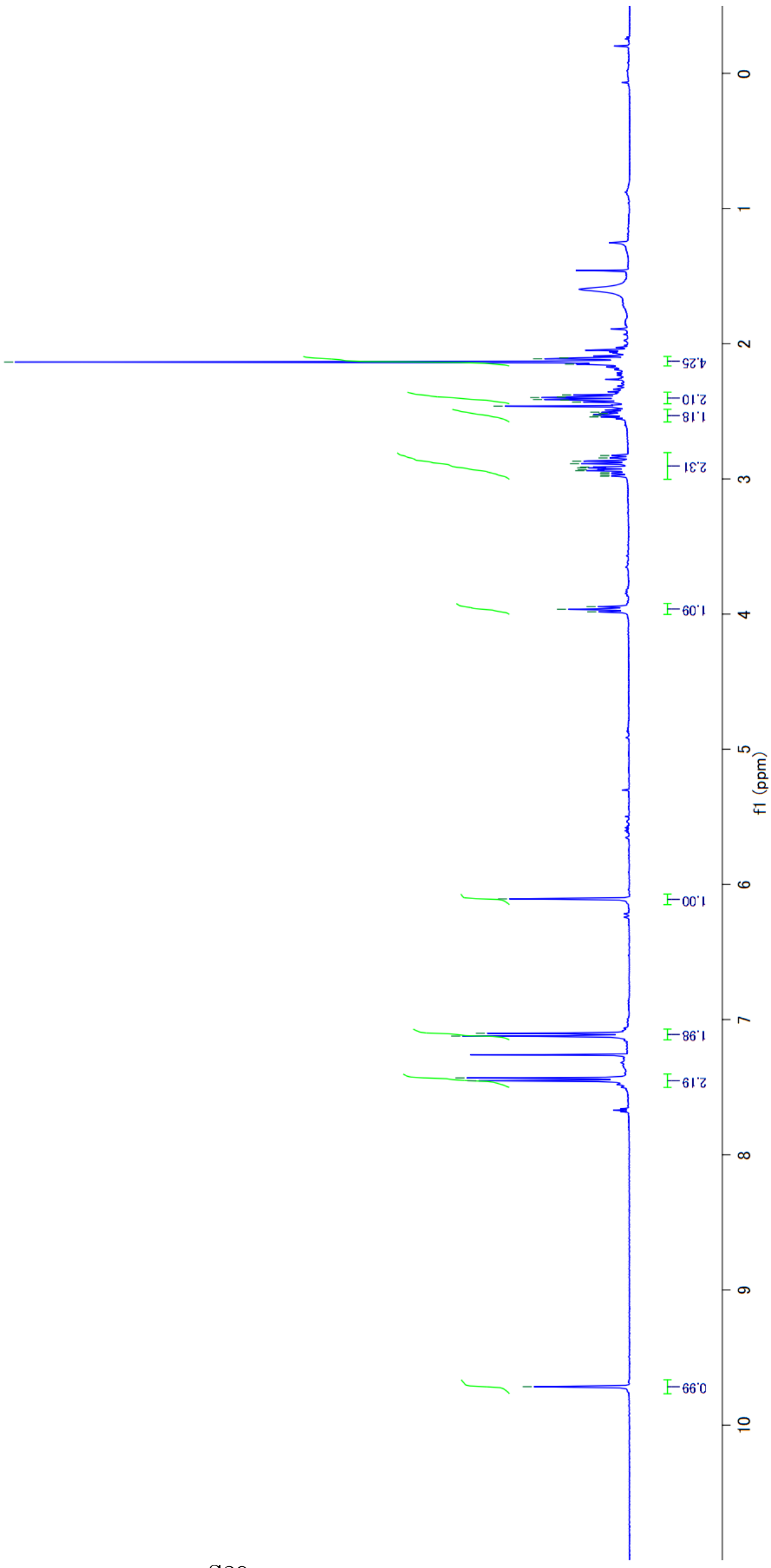

$101: L$
$221: L$

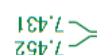

$91<6-$

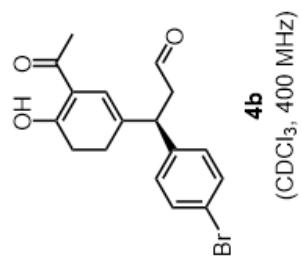


$8961-$
$16.62-$
$109 \varepsilon-$
$629 b=$
$90.2 b-$

$\angle 8 \angle 01-$

$86811-$
$26.021-$

6ะ6孔1-

$88^{\circ}|\varepsilon|>$
$60^{\circ} \mathrm{ZEI}$

$89^{\circ} 0 t 1-$

0ะ $6 \angle 1-$

$\angle 0661=$
$96002=$

य>

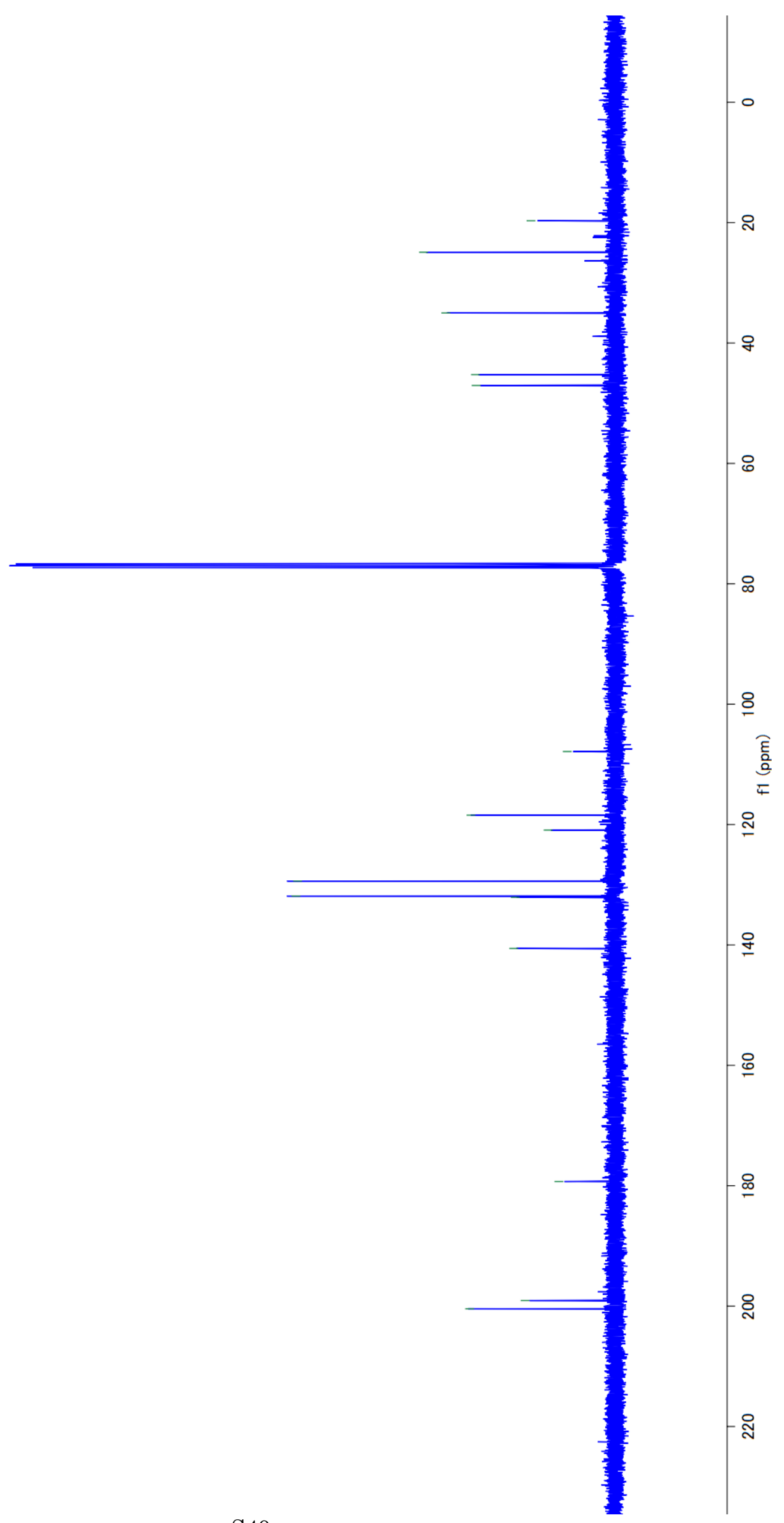

$\mathrm{S} 40$ 


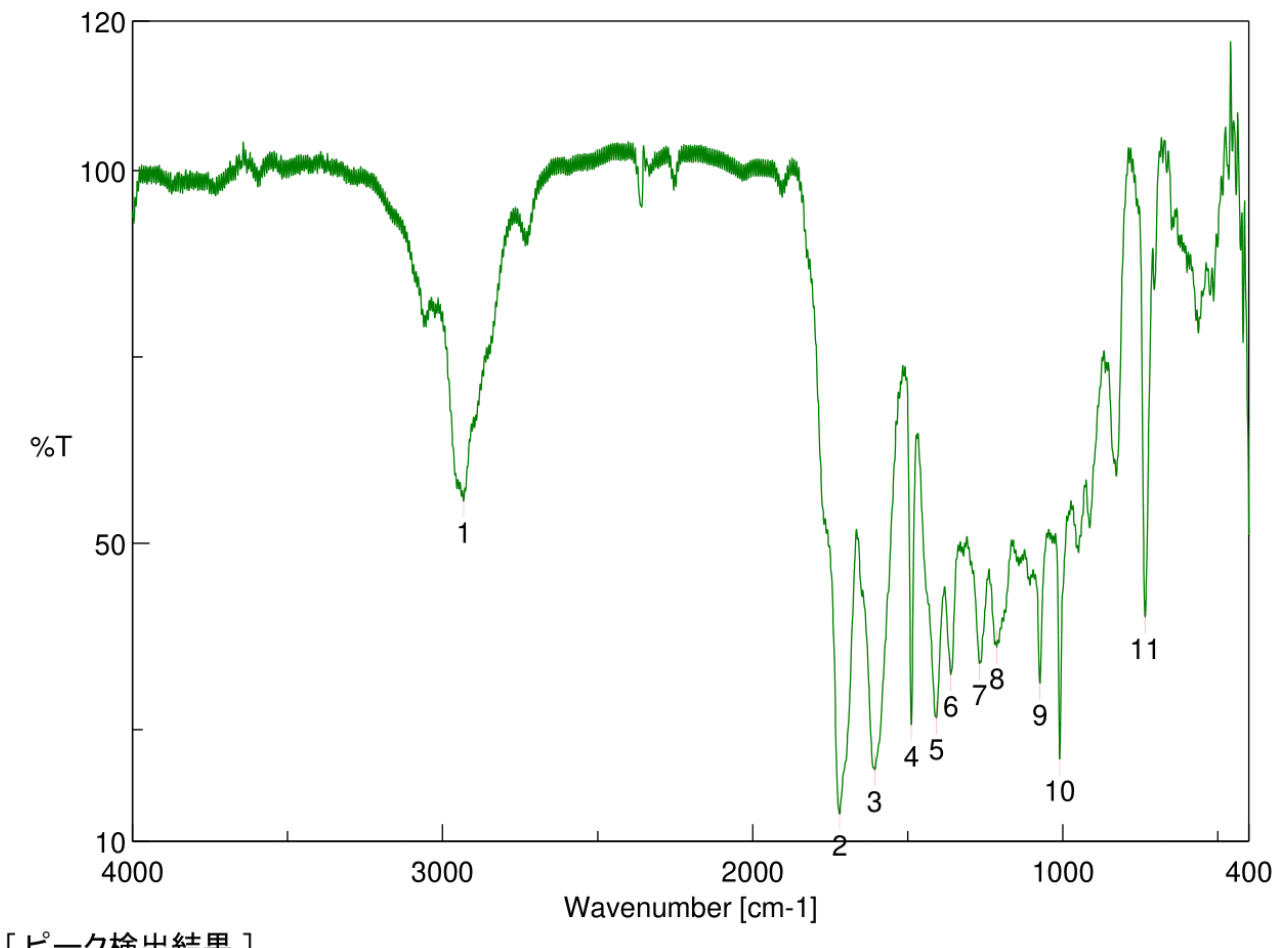

\begin{tabular}{lll}
\multicolumn{2}{l}{ [ピーク検出結果 ] } \\
No. & 位置 & 強度 \\
1 & 2932.23 & 55.6464 \\
3 & 1606.41 & 19.621 \\
5 & 1406.82 & 26.5488 \\
7 & 1268.93 & 33.8394 \\
9 & 1074.16 & 31.1721 \\
11 & 734.746 & 40.0587
\end{tabular}

$\begin{array}{lll}\text { No. } & \text { 位置 } & \text { 強度 } \\ 2 & 1720.19 & 13.6789 \\ 4 & 1488.78 & 25.5988 \\ 6 & 1361.5 & 32.3713 \\ 8 & 1213.01 & 36.0521 \\ 10 & 1009.55 & 21.013\end{array}$

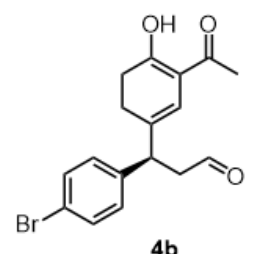




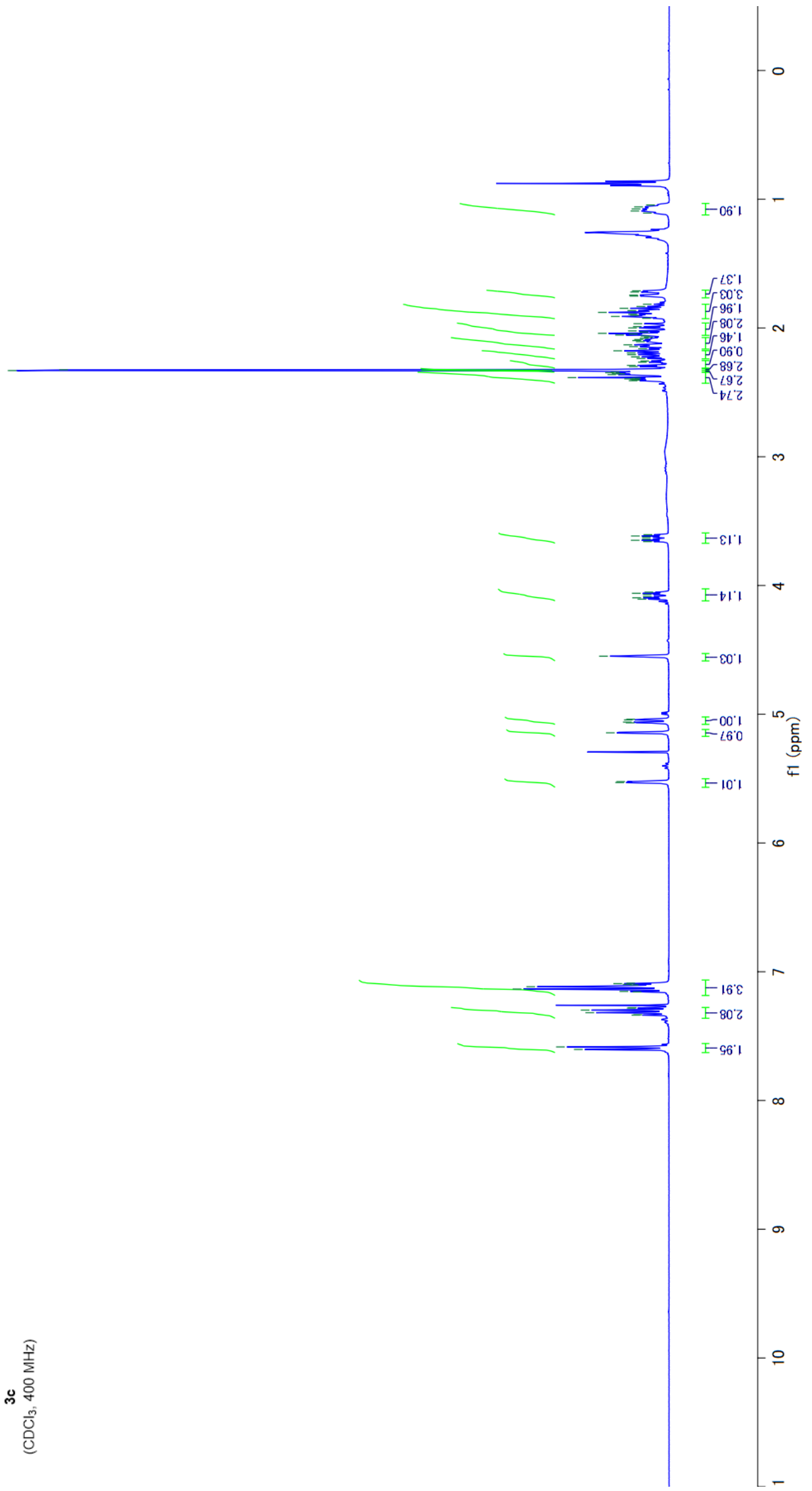


$\angle 8^{\circ}+11$
$99^{\circ} \mathrm{i}$

${ }_{00^{\circ}, t z}^{96 z}>$

0162

to. 18

ธะ่า

89 '

$1299-$

$112 t-$

$16.16-$

$12^{\prime} 96$ -

${ }^{89} 801>$

$69^{\circ}+2 \mathrm{l}$

SO'GZ1-

EI LCI]

GELदा]

IE'Bz1-

$8+821$ -

$\angle 8821$

૪દ'દા Ј

L8.681

Es.0 1 -

$67 ' 981$
$8 t^{\circ} 981$

ttooz-

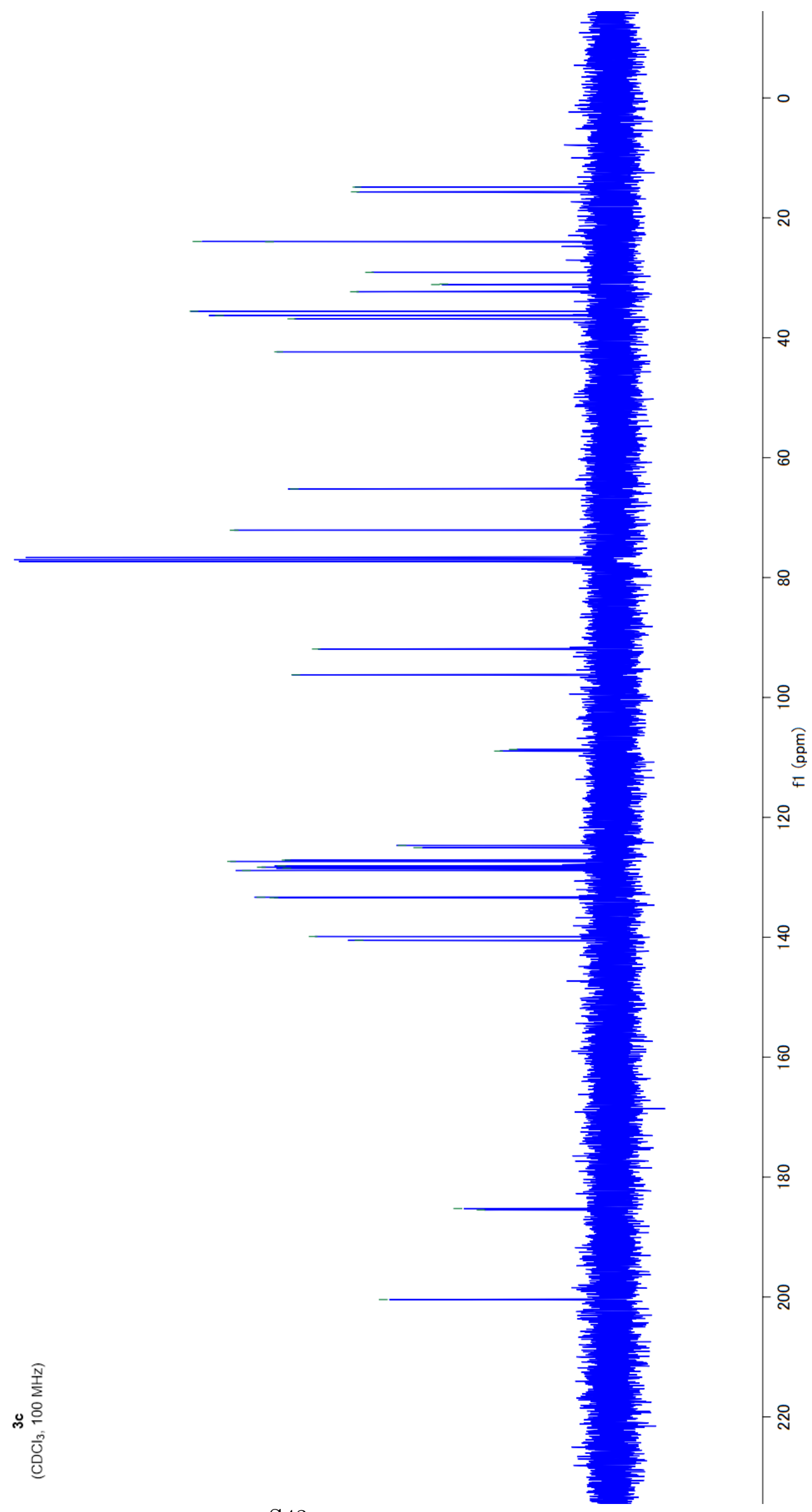

(1) 


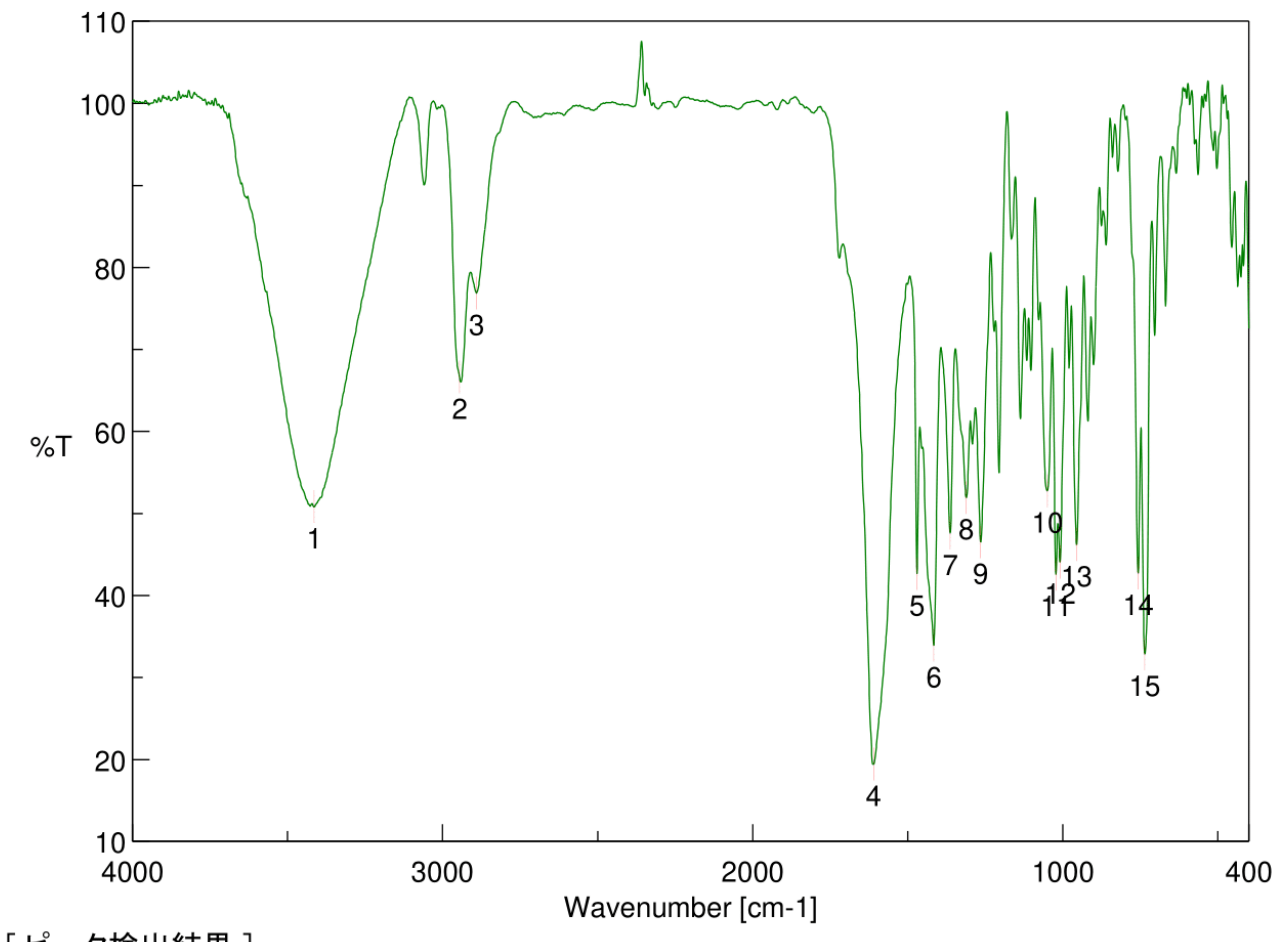

\begin{tabular}{lll}
\multicolumn{3}{l}{ [ピーク検出結果 ] } \\
No. & 位置 & 強度 \\
1 & 3414.35 & 50.7813 \\
3 & 2890.77 & 76.8517 \\
5 & 1470.46 & 42.6395 \\
7 & 1363.43 & 47.5706 \\
9 & 1265.07 & 46.5094 \\
11 & 1022.09 & 42.5559 \\
13 & 955.555 & 46.1624 \\
15 & 735.71 & 32.8542
\end{tabular}

No. 位置強度

$2 \quad 2945.73 \quad 66.6051$

$\begin{array}{lll}4 & 1610.27 & 19.3868\end{array}$

$\begin{array}{lll}6 & 1416.46 & 33.8778\end{array}$

$8 \quad 1311.36 \quad 51.9259$

$\begin{array}{lll}10 & 1050.05 & 52.7309\end{array}$

$\begin{array}{lll}12 & 1008.59 & 44.0351\end{array}$

$\begin{array}{lll}14 & 756.923 & 42.7093\end{array}$

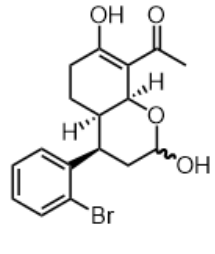

$3 c$ 


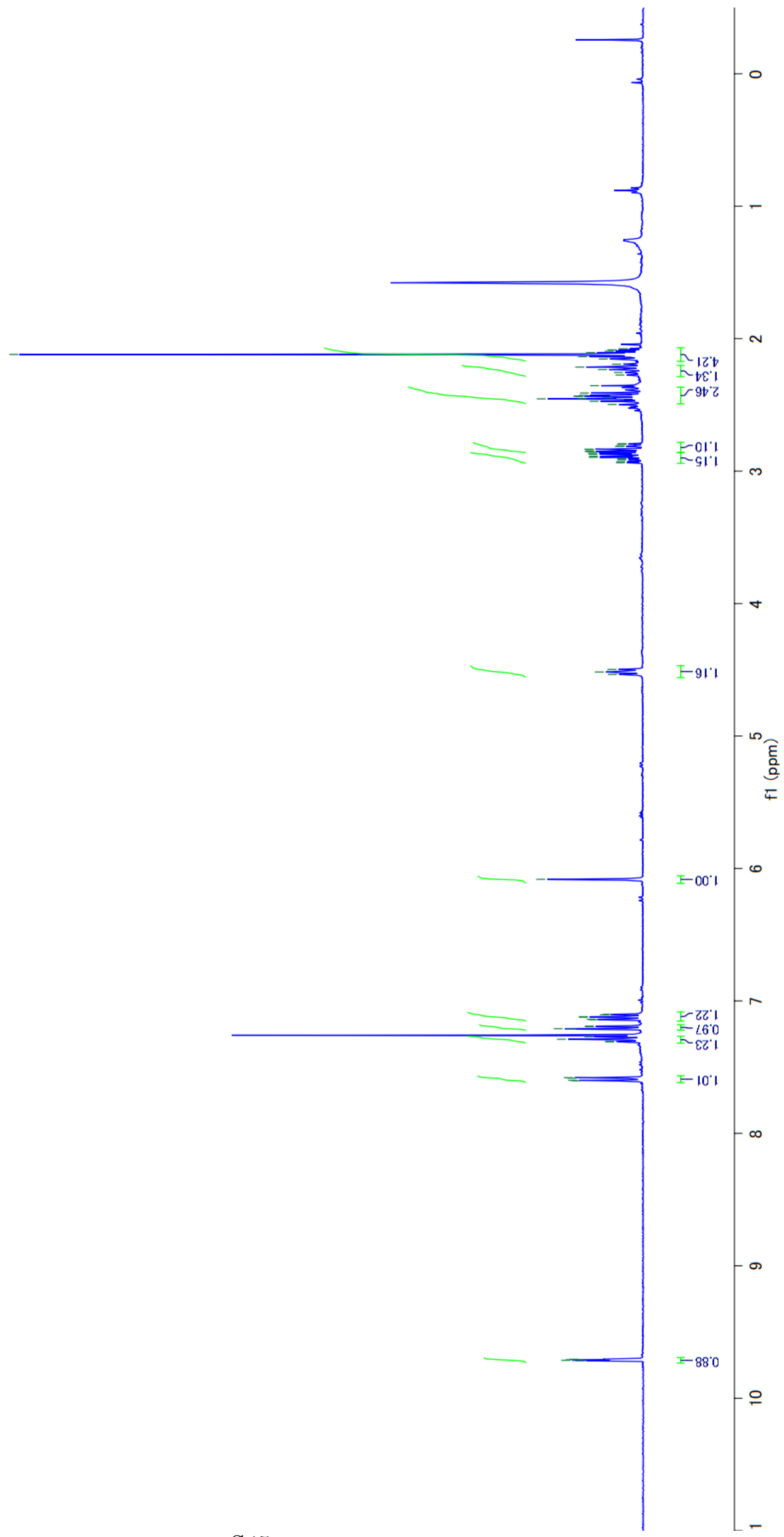

S0 $\left.L^{\circ} 6\right]$

$60 t 67$

E1:6

〈य 
$\angle 961-$

s9. 9 -

$10^{\circ} \mathrm{\varepsilon}-$

Lt $t 50-$
$86.95-$

$26.201-$

$97611-$

$86^{\circ}$ เ乙1

$96821>$

6द $8 \mathrm{CZ}$

6ช้ เยเ

LtOt

$2 t 6 L 1-$

$60^{\circ} 661=$
$0 L^{\circ} 002-$

$\sum_{-1}^{\infty}$
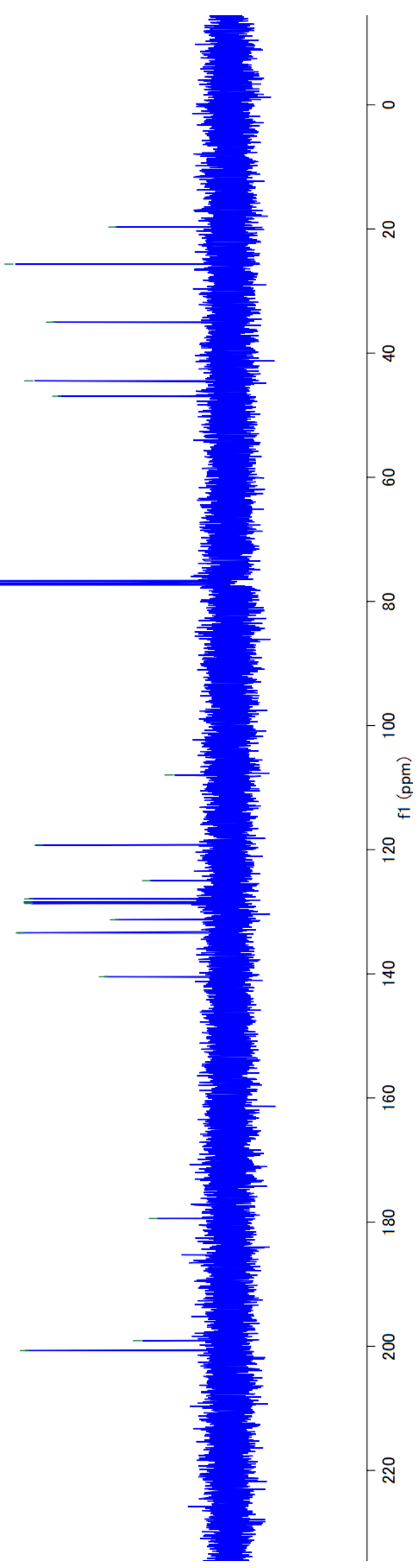


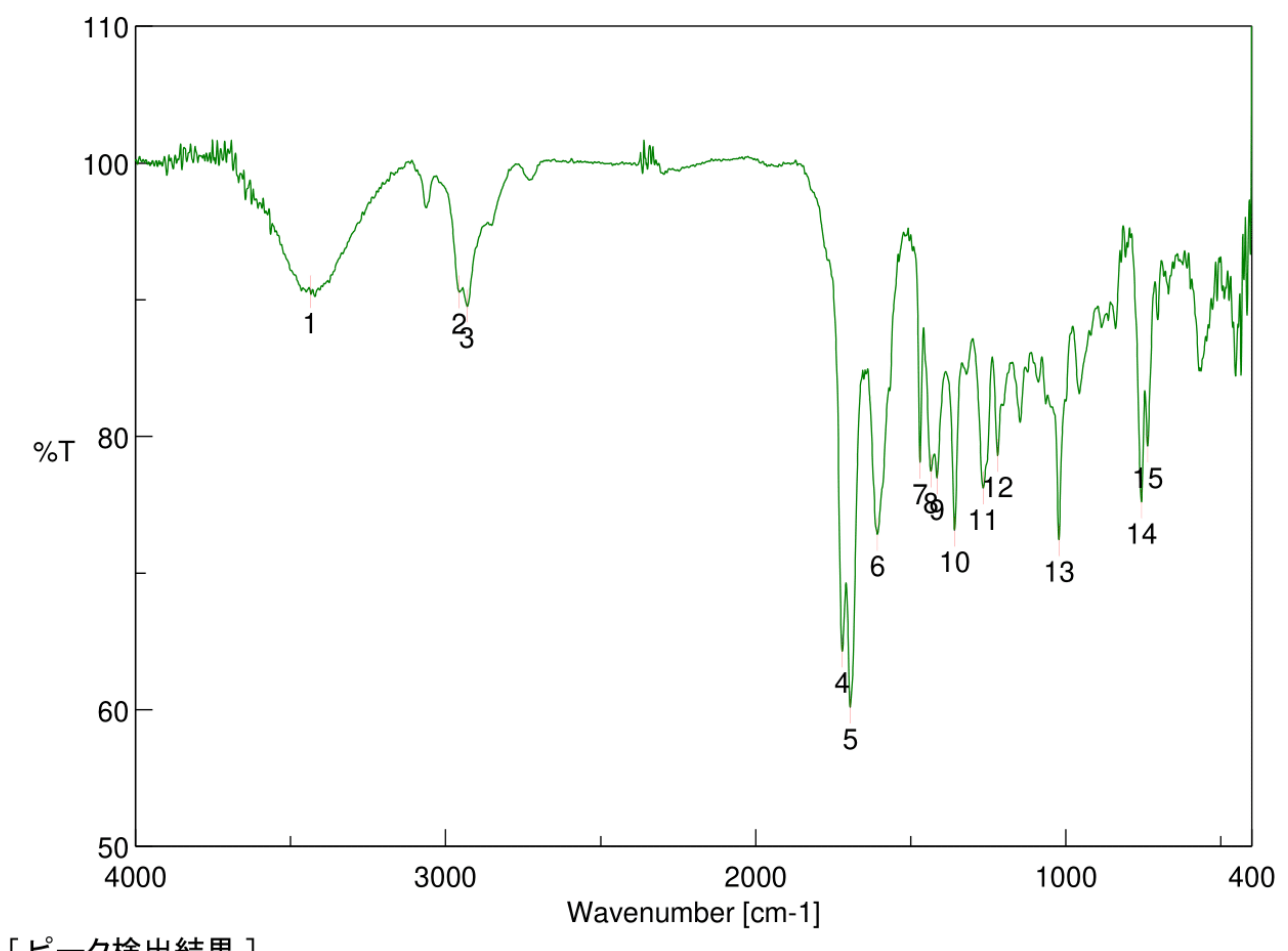

\begin{tabular}{lll}
\multicolumn{3}{l}{ [ピーク検出結果 ] } \\
No. & 位置 & 強度 \\
1 & 3436.53 & 90.5623 \\
3 & 2931.27 & 89.5262 \\
5 & 1695.12 & 60.178 \\
7 & 1470.46 & 78.0872 \\
9 & 1415.49 & 76.9502 \\
11 & 1266.04 & 76.2188 \\
13 & 1022.09 & 72.418 \\
15 & 735.71 & 79.2809
\end{tabular}

No. 位置強度

$2 \quad 2956.34 \quad 90.5548$

$4 \quad 1721.16 \quad 64.277$

$\begin{array}{lll}6 & 1607.38 & 72.8206\end{array}$

$\begin{array}{lll}8 & 1434.78 & 77.4411\end{array}$

$\begin{array}{lll}10 & 1358.6 & 73.1237\end{array}$

$\begin{array}{lll}12 & 1219.76 & 78.577\end{array}$

$\begin{array}{lll}14 & 755.959 & 75.1879\end{array}$

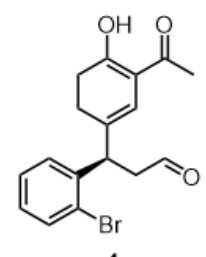

$4 c$ 

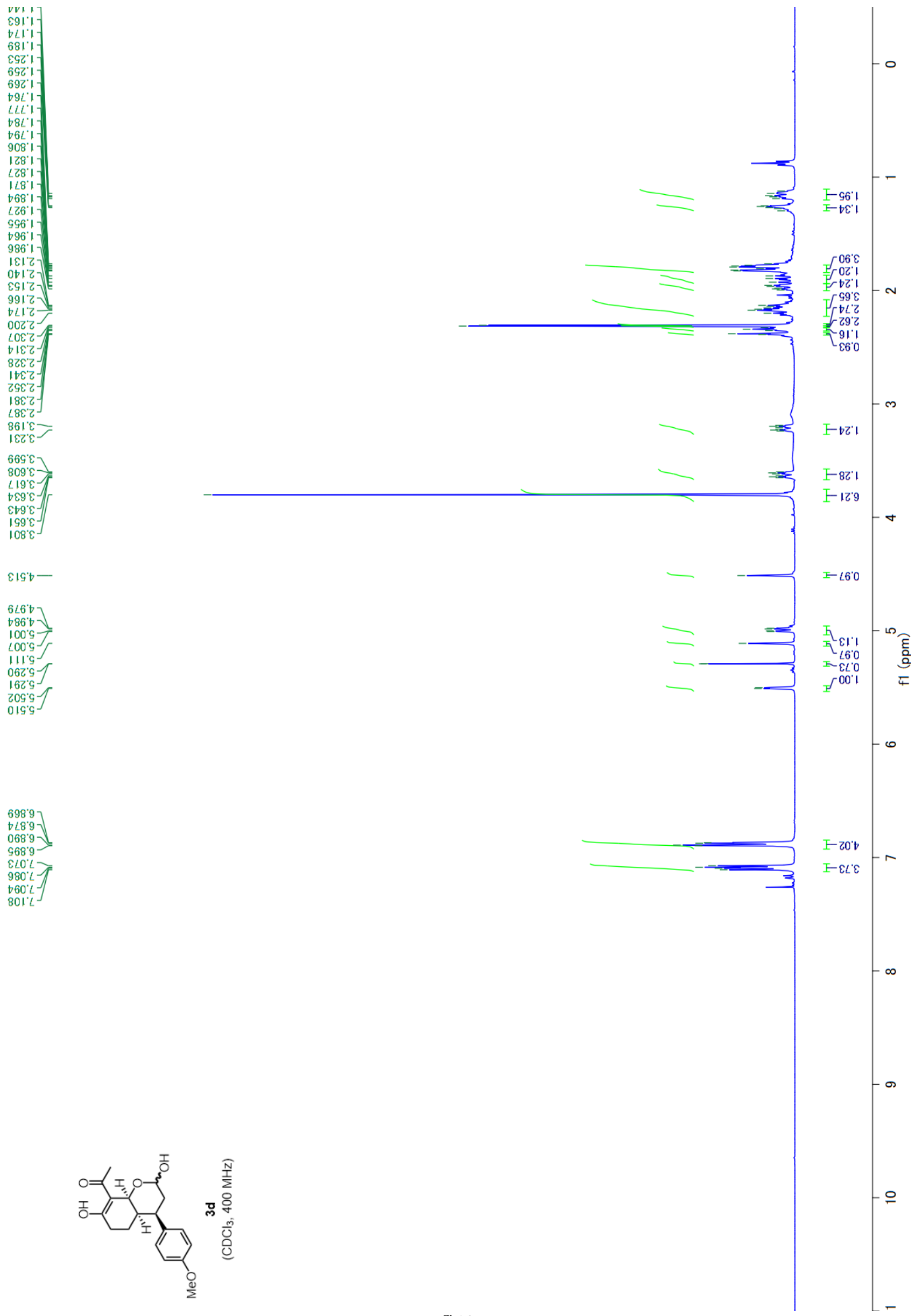
$\begin{array}{lc}L s^{\circ} \circ I & =\end{array}$

¿8'
$06^{\circ} \varepsilon z$

9062

$01.18>$

OZ'IE

ces

(c)

$66^{\circ} 68$

66.15

8199
61.99

५' $99-$

†ㄴㄷㅡ

$86 \cdot 16-$

Et 96 -

$29^{\circ} 801>$

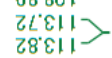

97821
$t 981$

6ระ

乙大 $\downarrow \varepsilon 1=$

90.891
81.891

$\left.\begin{array}{l}\left\lfloor E^{\prime} \subseteq 81\right. \\ \left\langle 9^{\circ} 981\right.\end{array}\right\rangle$

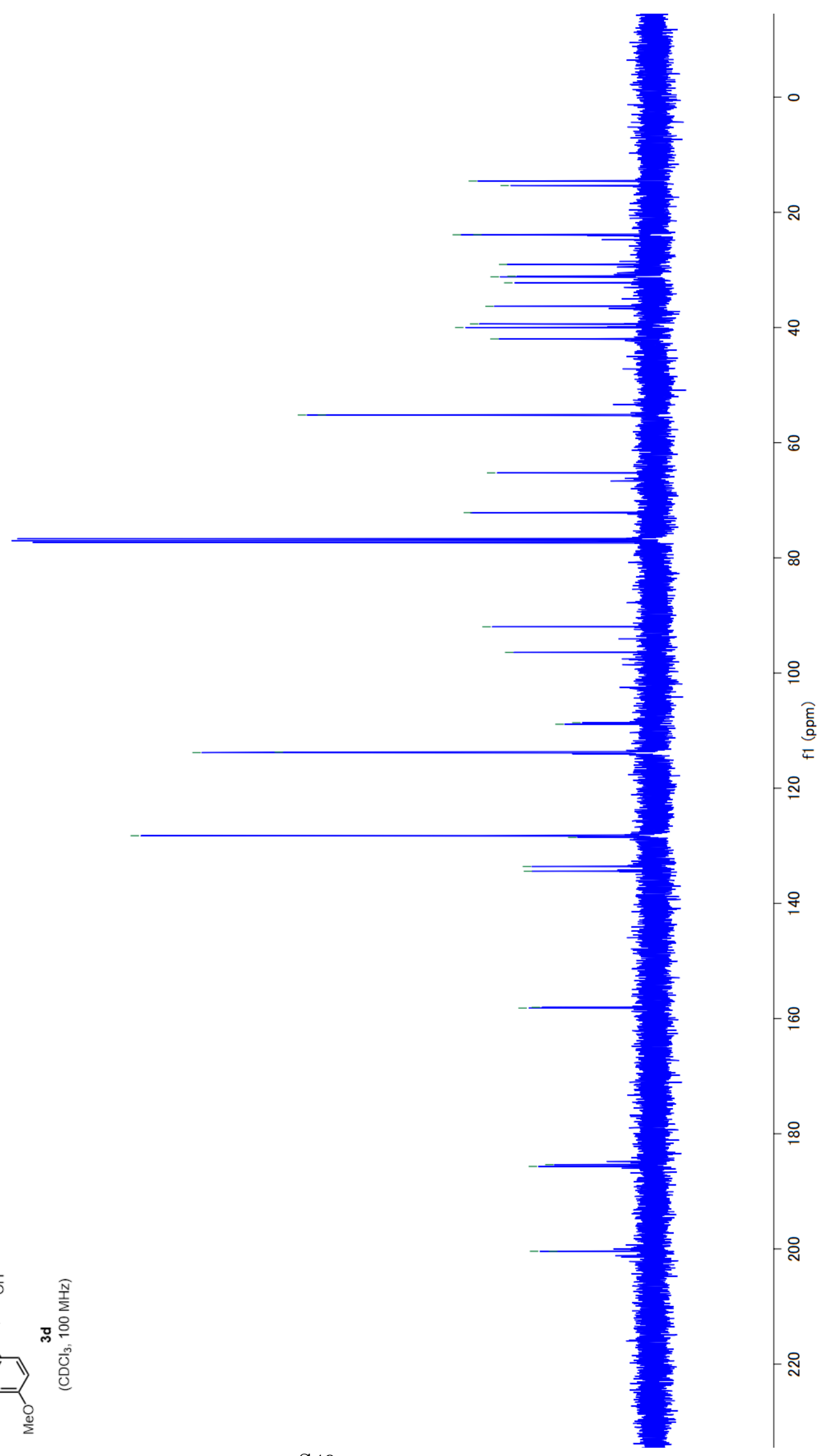

$00^{\circ} 002>$

(2) 


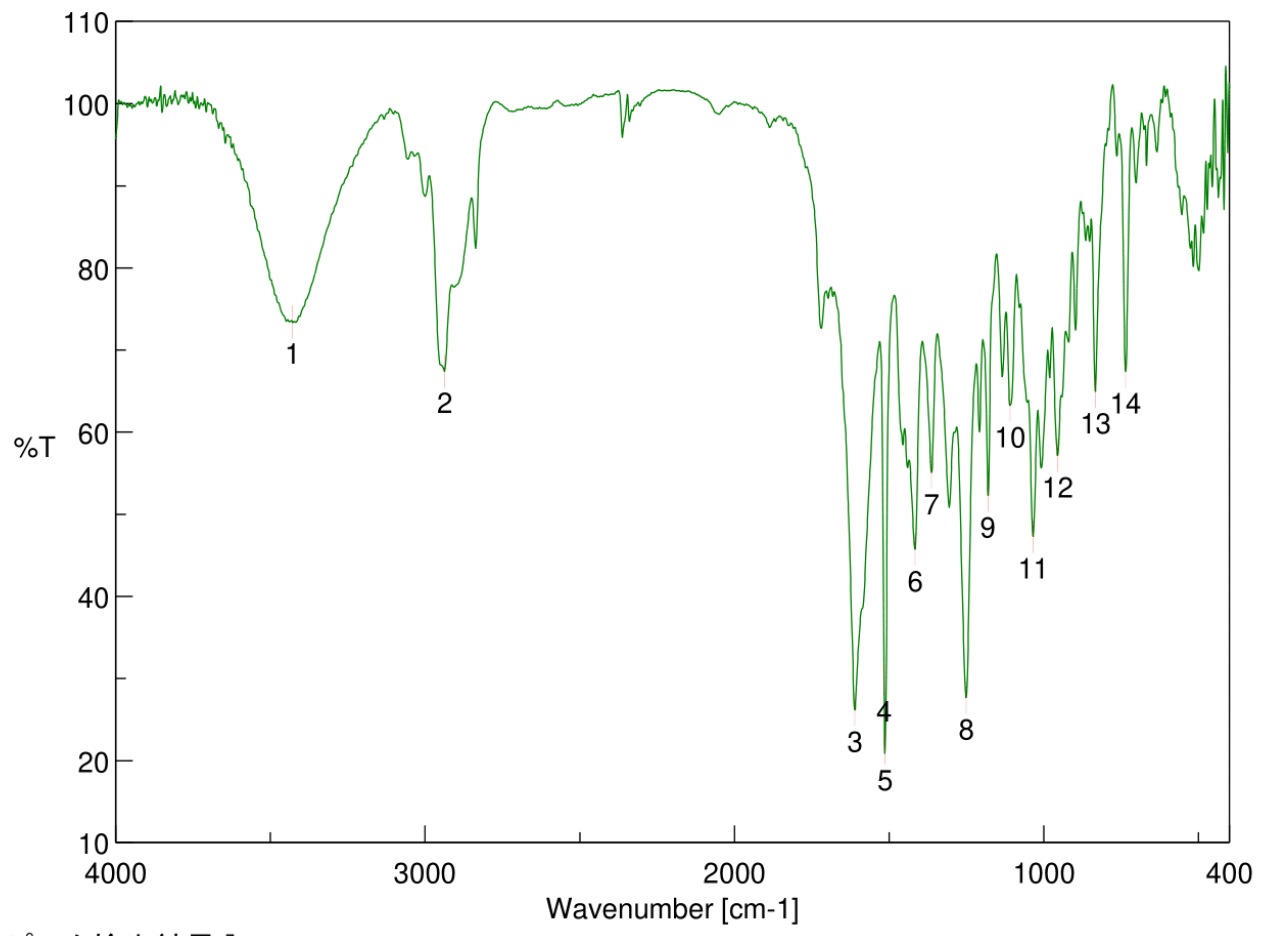

[ピーク検出結果 ]

$\begin{array}{llllll}\text { No. } & \text { 位置 } & \text { 強度 } & \text { No. } & \text { 位置 } & \text { 強度 } \\ 1 & 3428.81 & 73.3637 & 2 & 2937.06 & 67.3952 \\ 3 & 1611.23 & 26.1477 & 4 & 1516.74 & 29.8073 \\ 5 & 1512.88 & 21.4658 & 6 & 1416.46 & 45.6924 \\ 7 & 1363.43 & 55.0538 & 8 & 1250.61 & 27.6575 \\ 9 & 1180.22 & 52.2506 & 10 & 1108.87 & 63.2117 \\ 11 & 1034.62 & 47.2489 & 12 & 955.555 & 57.1211 \\ 13 & 833.098 & 64.9503 & 14 & 735.71 & 67.3652\end{array}$

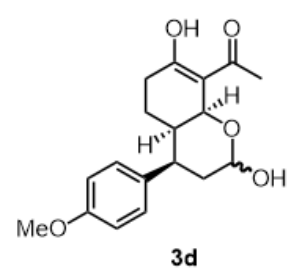



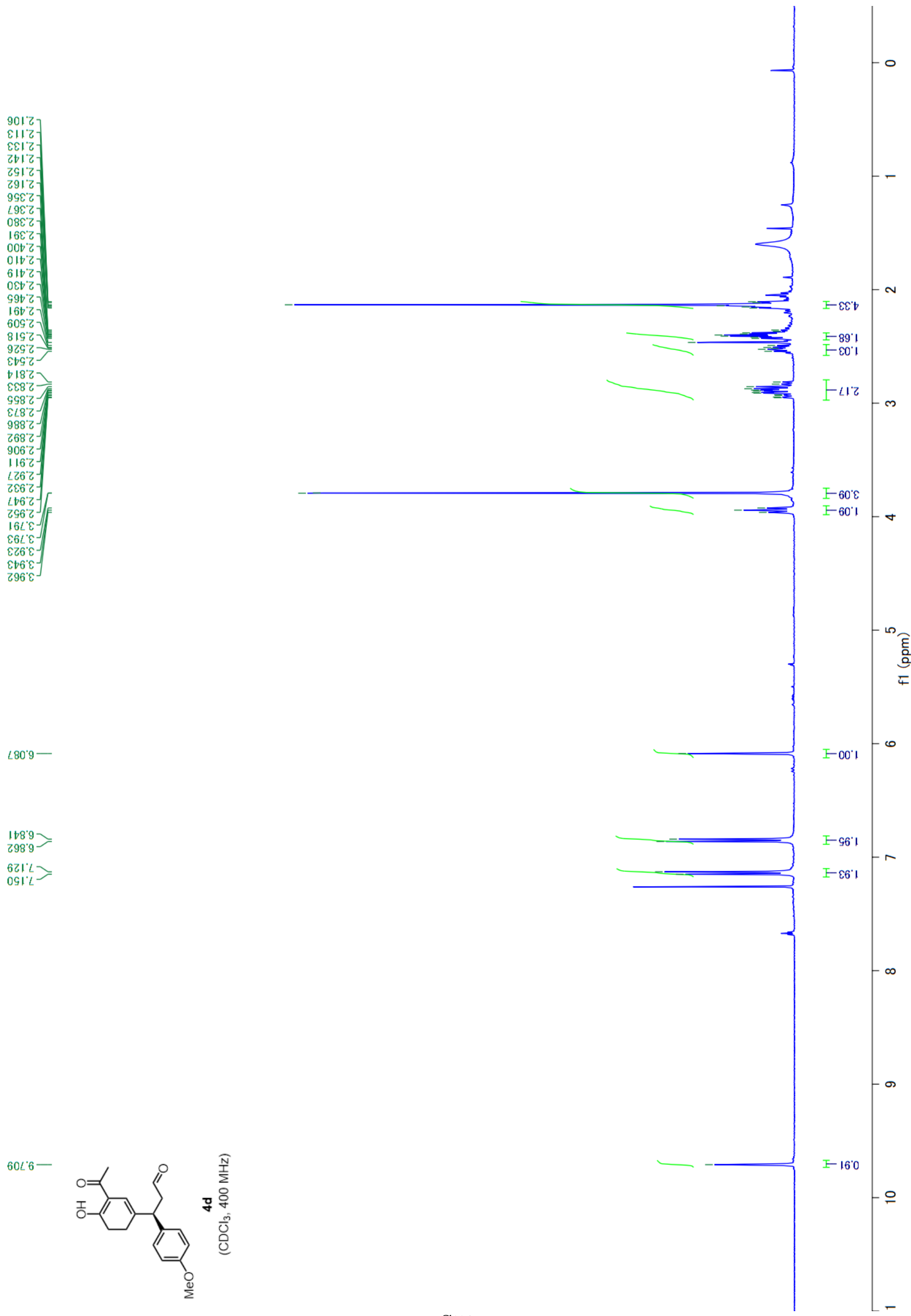

60l.6- 
$5961-$

L8toz-

$19 \varepsilon$

El $s t-$
$6 t^{\prime}: b-$

เฉ $s 9$

$66^{\circ} \mathrm{LOI}-$

$01011-$

$D L L 11-$

$09821-$

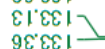

เร์ $661-$

य)

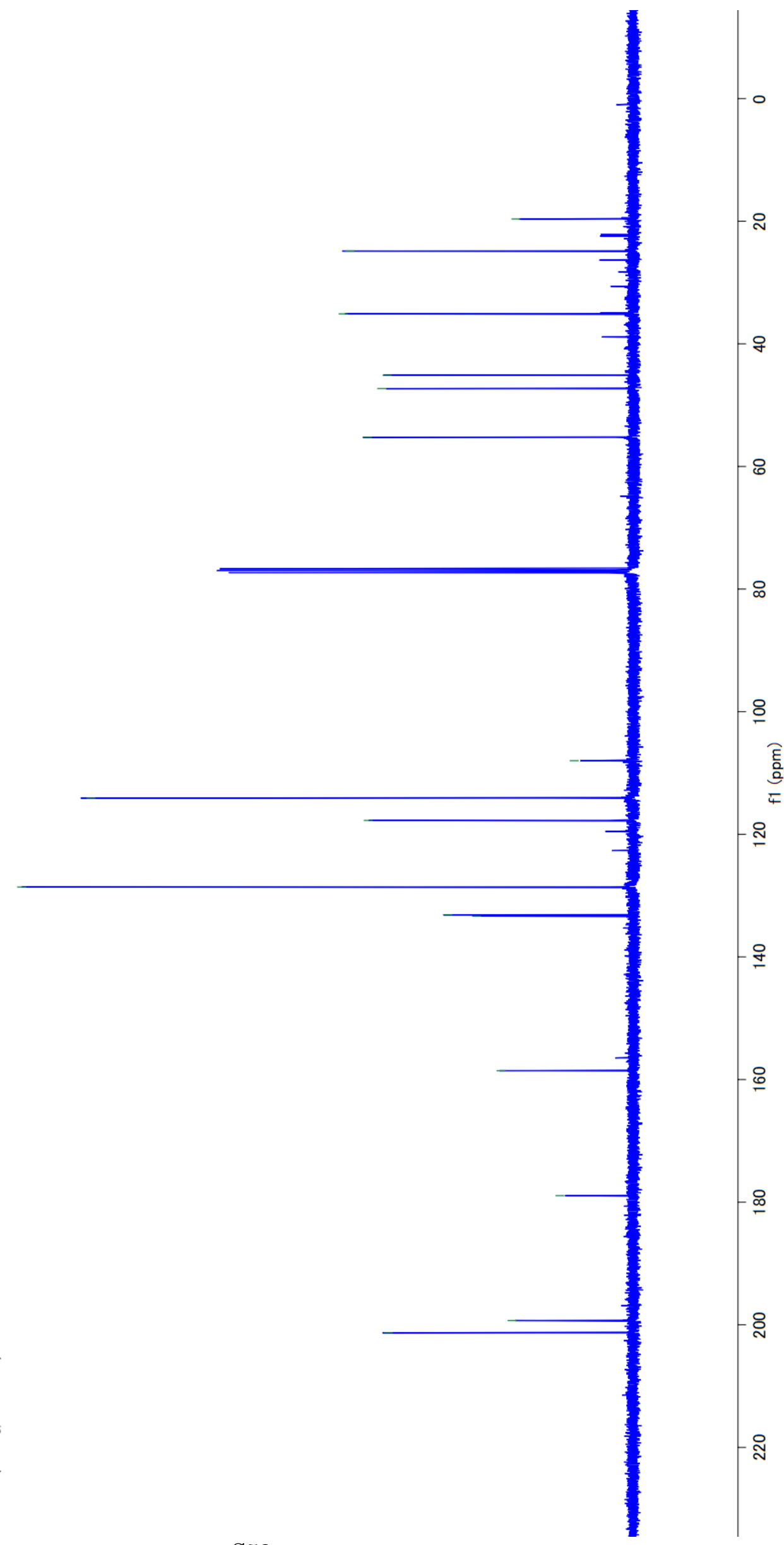

S52 


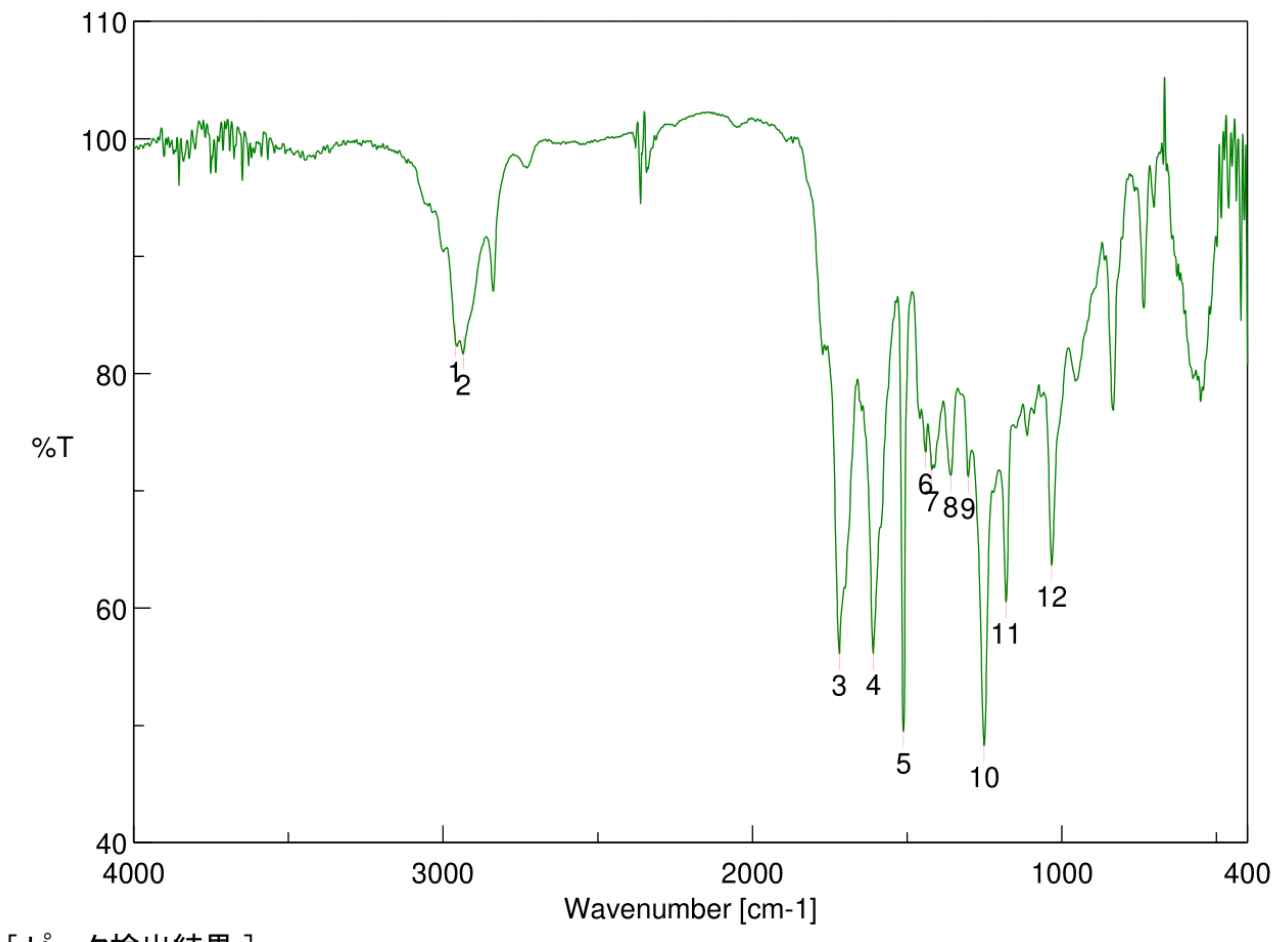

[ピーク検出結果 ]

$\begin{array}{llllll}\text { No. } & \text { 位置 } & \text { 強度 } & \text { No. } & \text { 位置 } & \text { 強度 } \\ 1 & 2960.2 & 82.7813 & 2 & 2934.16 & 81.7236 \\ 3 & 1719.23 & 56.1076 & 4 & 1609.31 & 56.1358 \\ 5 & 1511.92 & 49.4663 & 6 & 1440.56 & 73.3 \\ 7 & 1420.32 & 71.8062 & 8 & 1358.6 & 71.3013 \\ 9 & 1302.68 & 71.1909 & 10 & 1250.61 & 48.2685 \\ 11 & 1180.22 & 60.5101 & 12 & 1032.69 & 63.6617\end{array}$

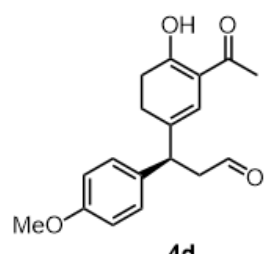




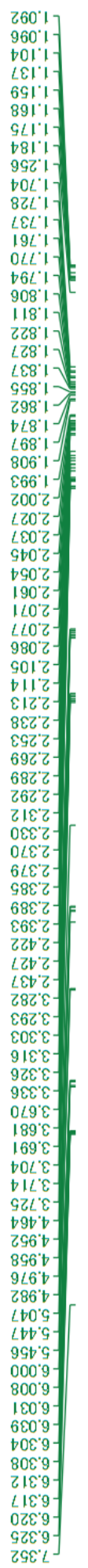

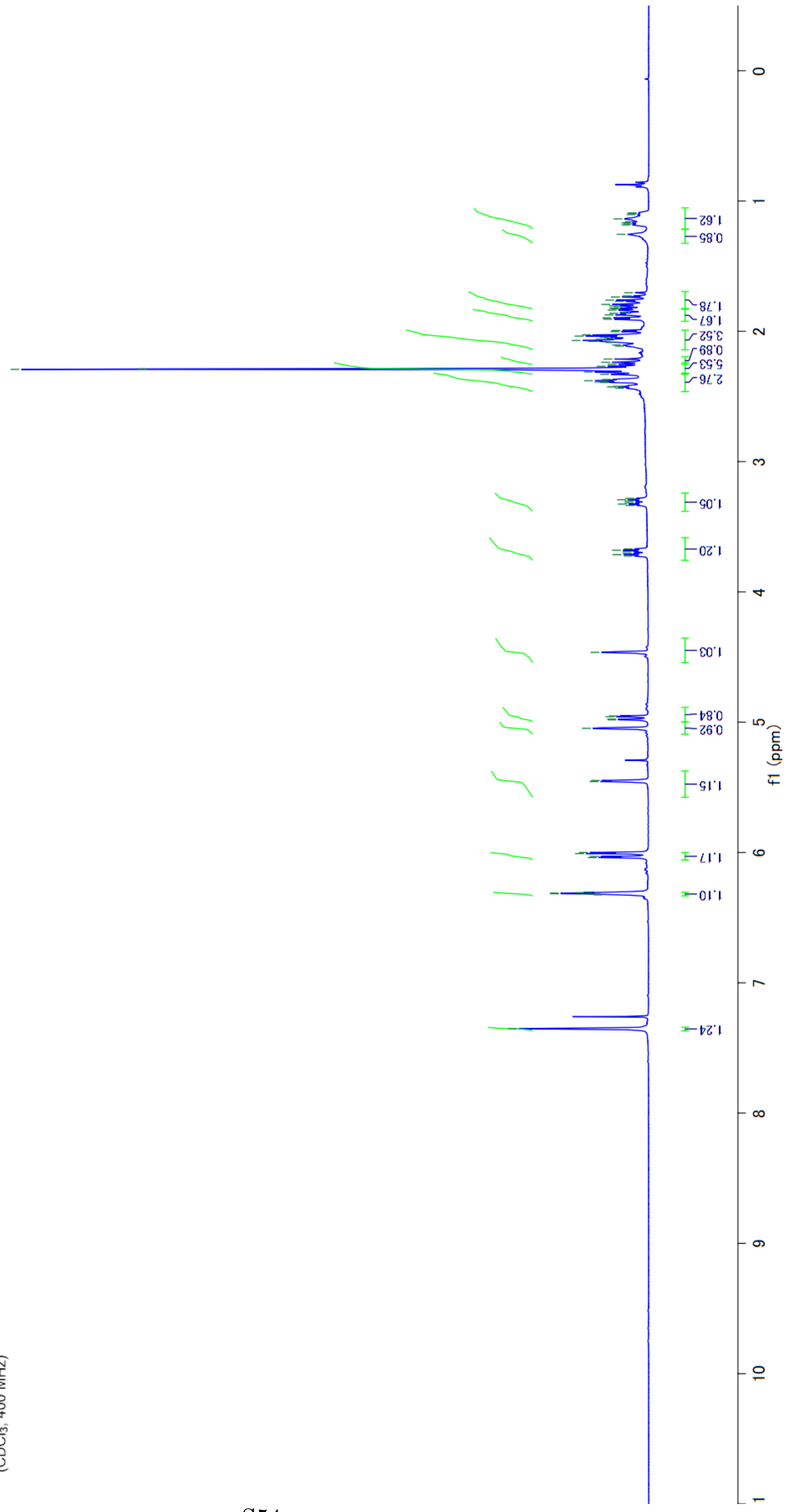




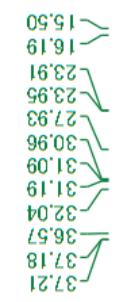

sะเ9-
६.।L-

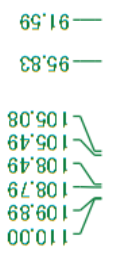

$b \varepsilon|b|$
$9 b|t|$

$\left\lfloor G^{\prime} G S 1\right.$
$\left.9 G^{\prime} 9\right)^{\prime}$

$80^{\circ} 981$
$89^{\circ} 981$

$\downarrow \mathcal{E}^{\circ} 002$
$\mathrm{G} 002$
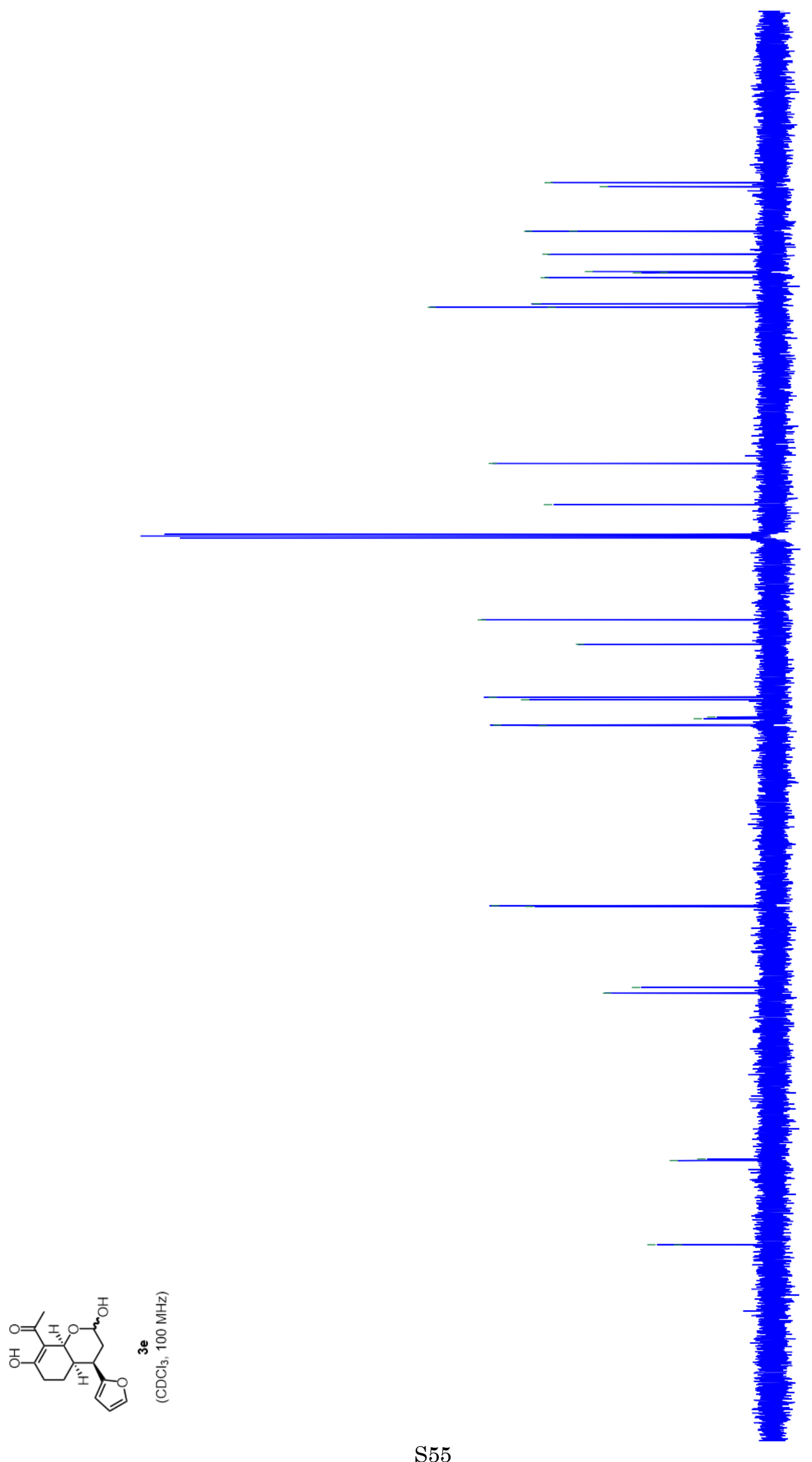

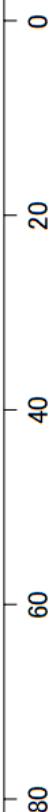

- 으

g

$\stackrel{8}{\circ}$

$\stackrel{\circ}{\circ}$

-웅

- ลิ

S55 


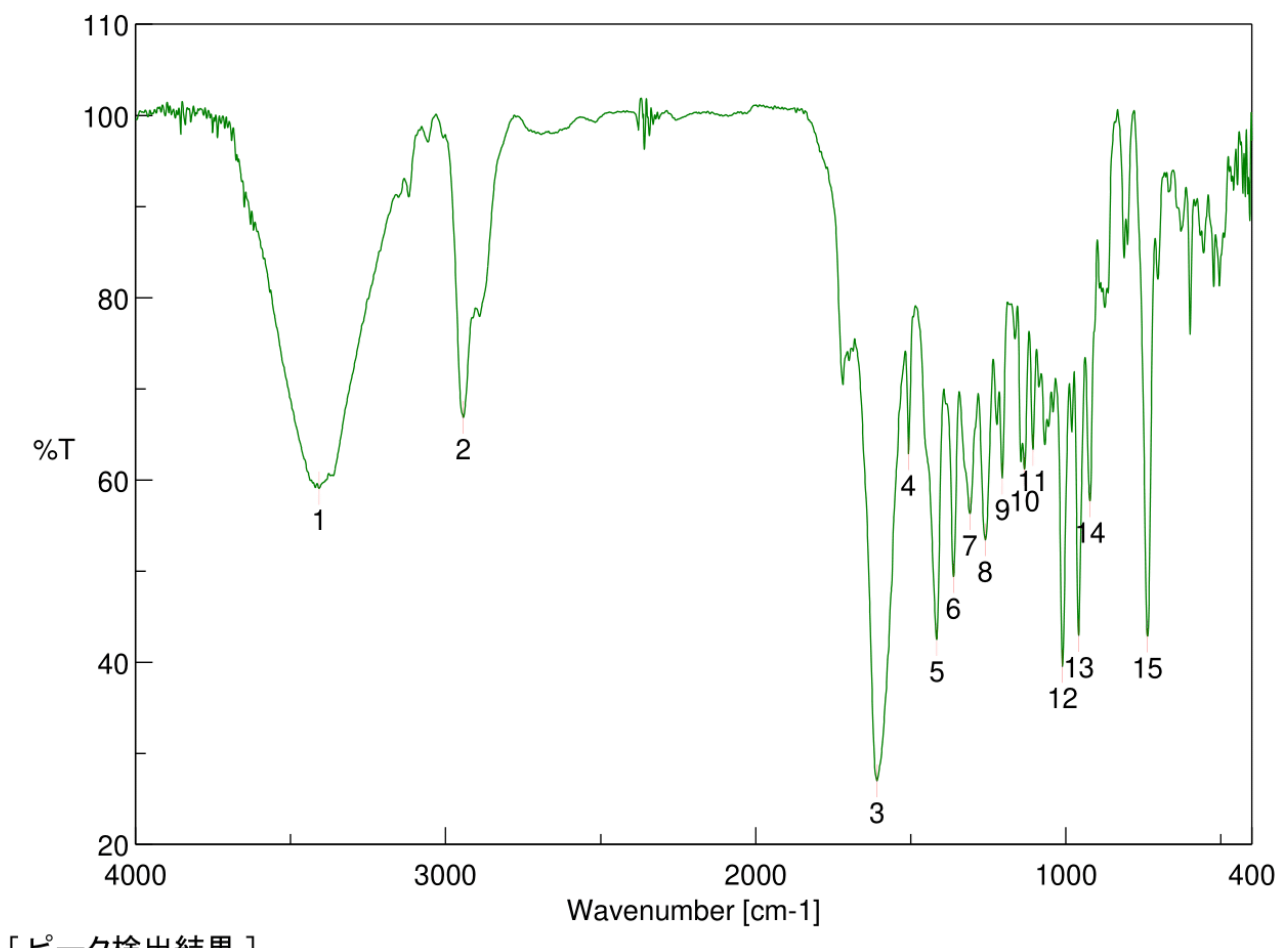

\begin{tabular}{llllll}
\multicolumn{2}{l}{ [ピーク検出結果 ] } & & & \\
No. & 位置 & 強度 & No. & 位置 & 強度 \\
1 & 3407.6 & 59.0756 & 2 & 2942.84 & 66.8518 \\
3 & 1609.31 & 26.9557 & 4 & 1507.1 & 62.8552 \\
5 & 1416.46 & 42.4817 & 6 & 1362.46 & 49.354 \\
7 & 1309.43 & 56.2923 & 8 & 1259.29 & 53.4063 \\
9 & 1205.29 & 60.1843 & 10 & 1132.97 & 61.176 \\
11 & 1105.98 & 63.351 & 12 & 1010.52 & 39.5005 \\
13 & 958.448 & 42.9247 & 14 & 921.807 & 57.667 \\
15 & 736.674 & 42.8308 & & &
\end{tabular}

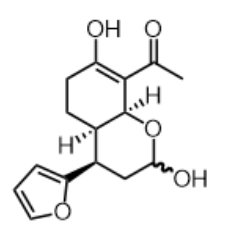

$3 e$ 


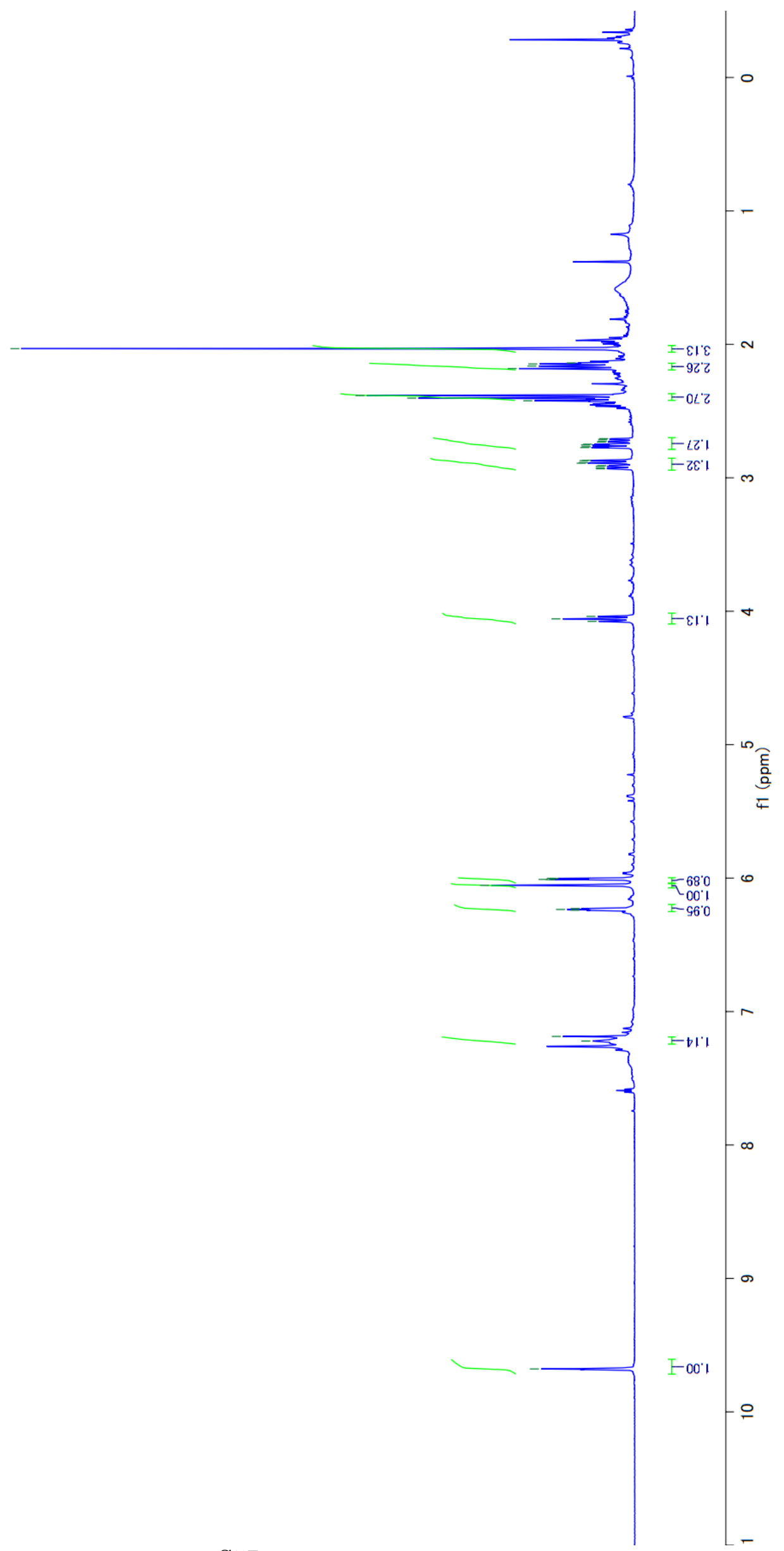


ILEZ

$90.58-1$
$68^{\circ} 88-$
91.00
$66 . t 6 า$

$81.901=$
$06.201=$
$08011=$

$59611-$

11.081-

$68|1| 1-$

$09+691-$

$916 L 1-$

s. $661=$
$8 b^{\circ} 002-$
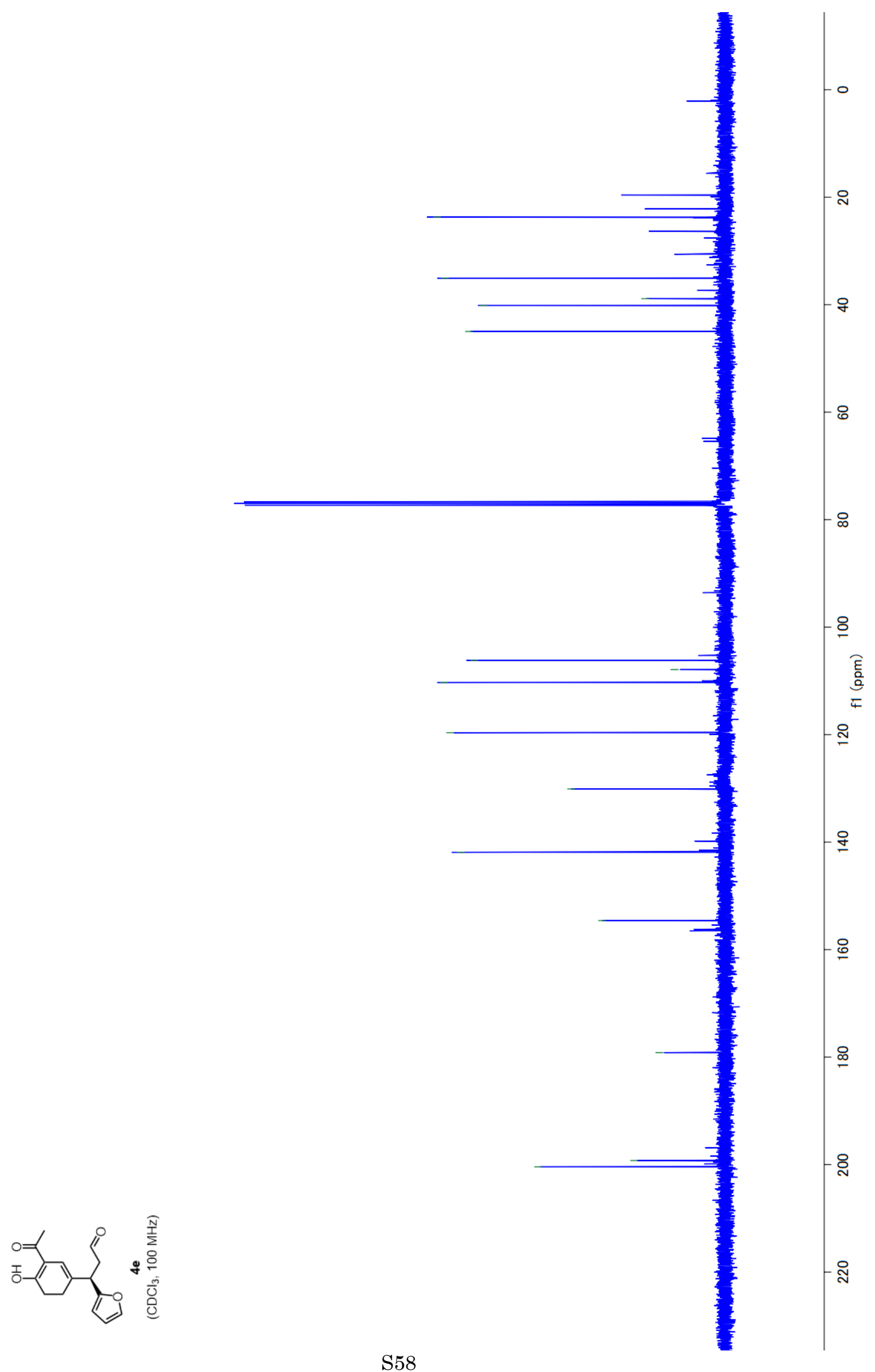


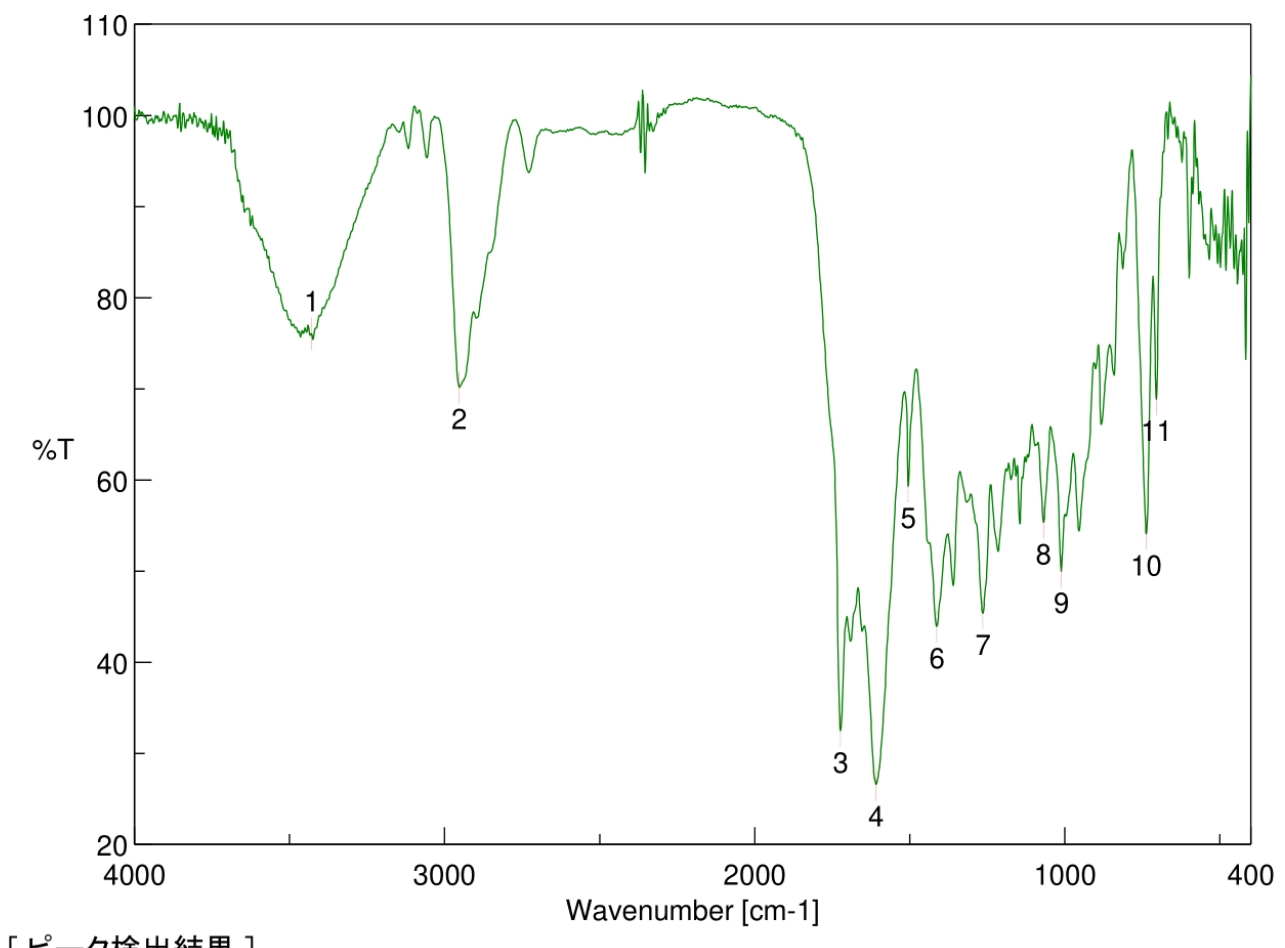

\begin{tabular}{lll}
\multicolumn{3}{l}{ [ピーク検出結果 ] } \\
No. & 位置 & 強度 \\
1 & 3428.81 & 76.0102 \\
3 & 1723.09 & 32.4828 \\
5 & 1505.17 & 59.2893 \\
7 & 1264.11 & 45.3492 \\
9 & 1011.48 & 49.9716 \\
11 & 704.855 & 68.8501
\end{tabular}

No. 位置強度

$2 \quad 2952.48 \quad 70.1498$

$\begin{array}{lll}4 & 1608.34 & 26.5652\end{array}$

$\begin{array}{lll}6 & 1412.6 & 43.9151\end{array}$

$\begin{array}{lll}8 & 1068.37 & 55.3129\end{array}$

$\begin{array}{lll}10 & 737.639 & 54.0767\end{array}$

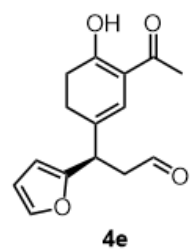



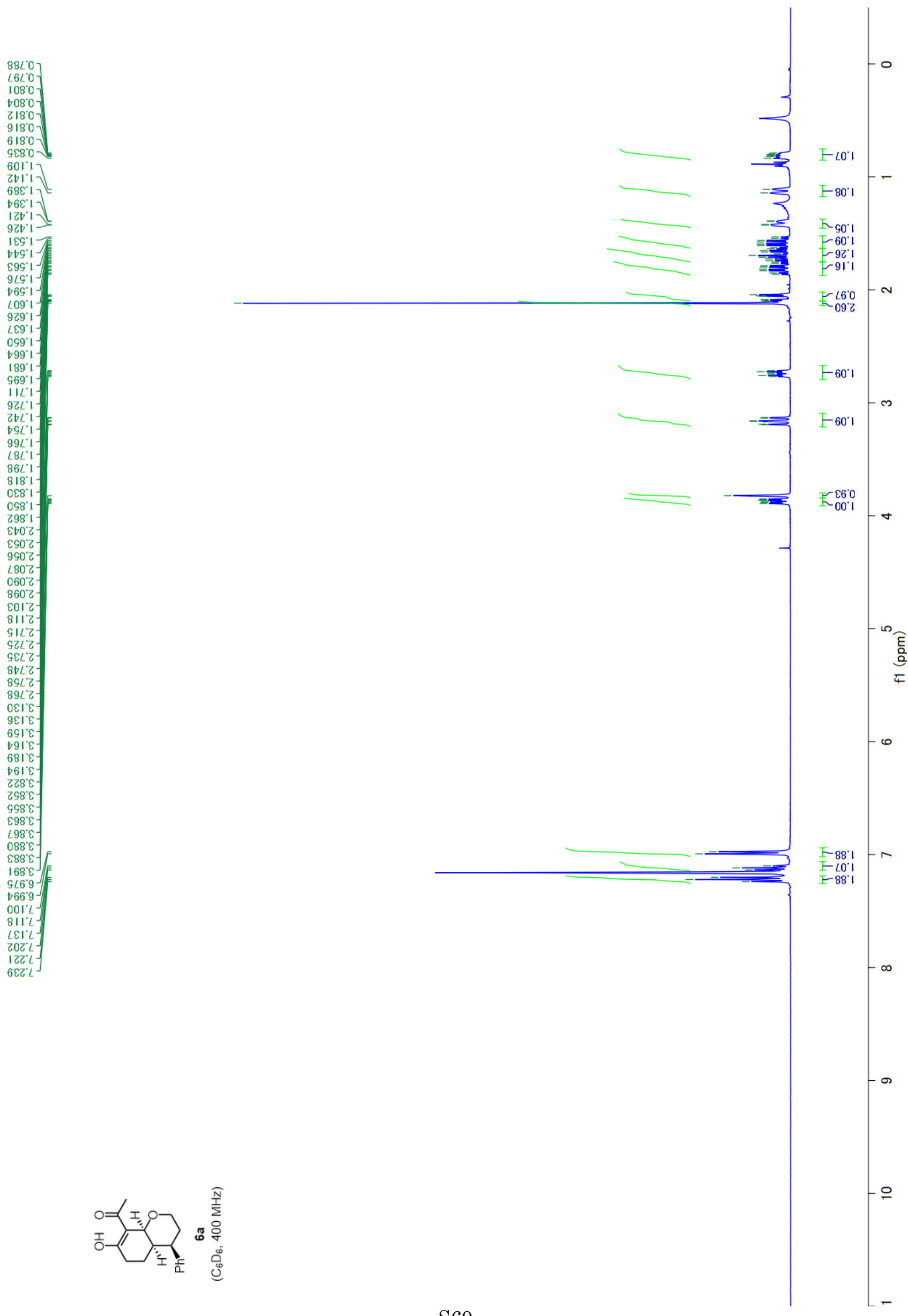


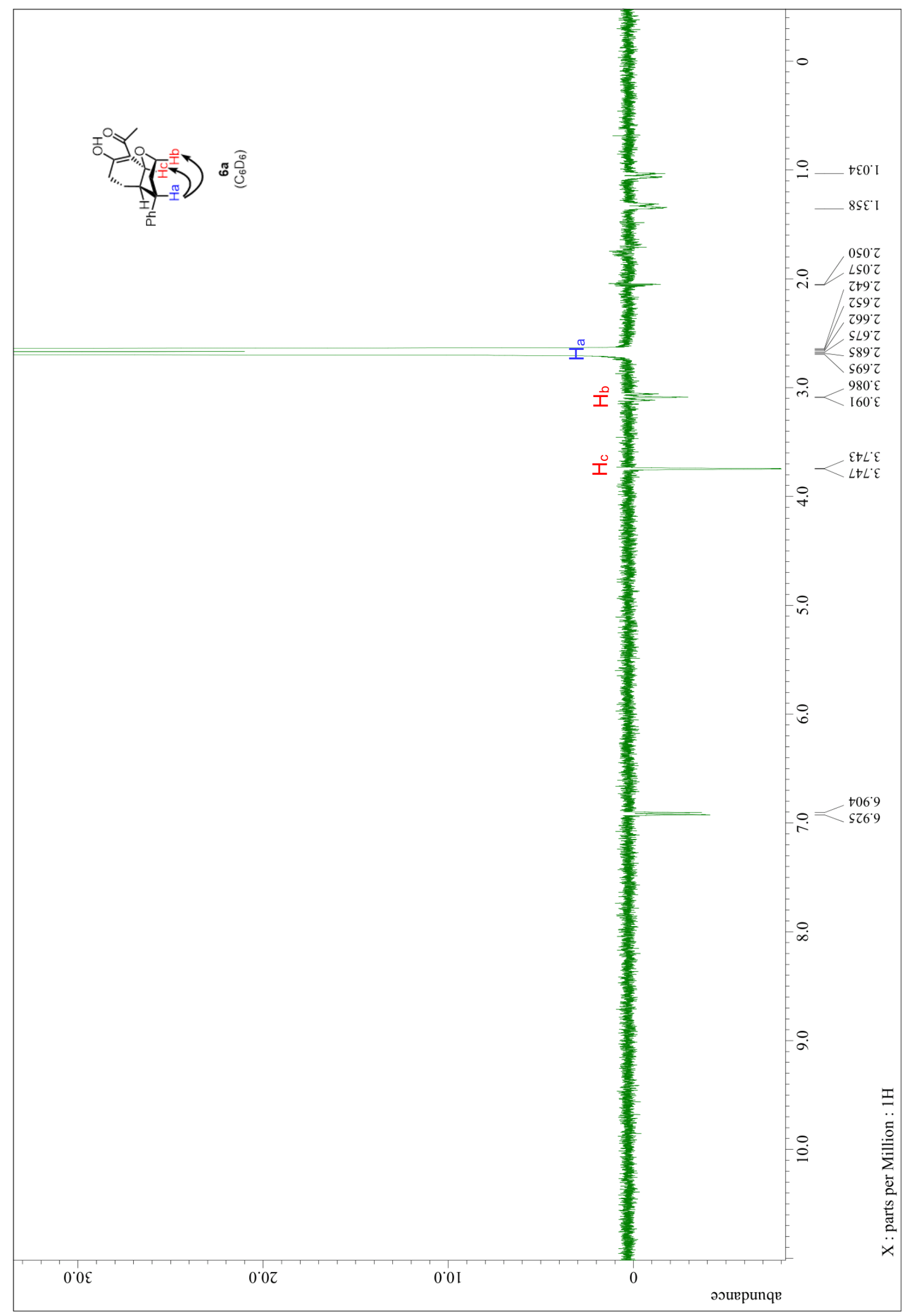




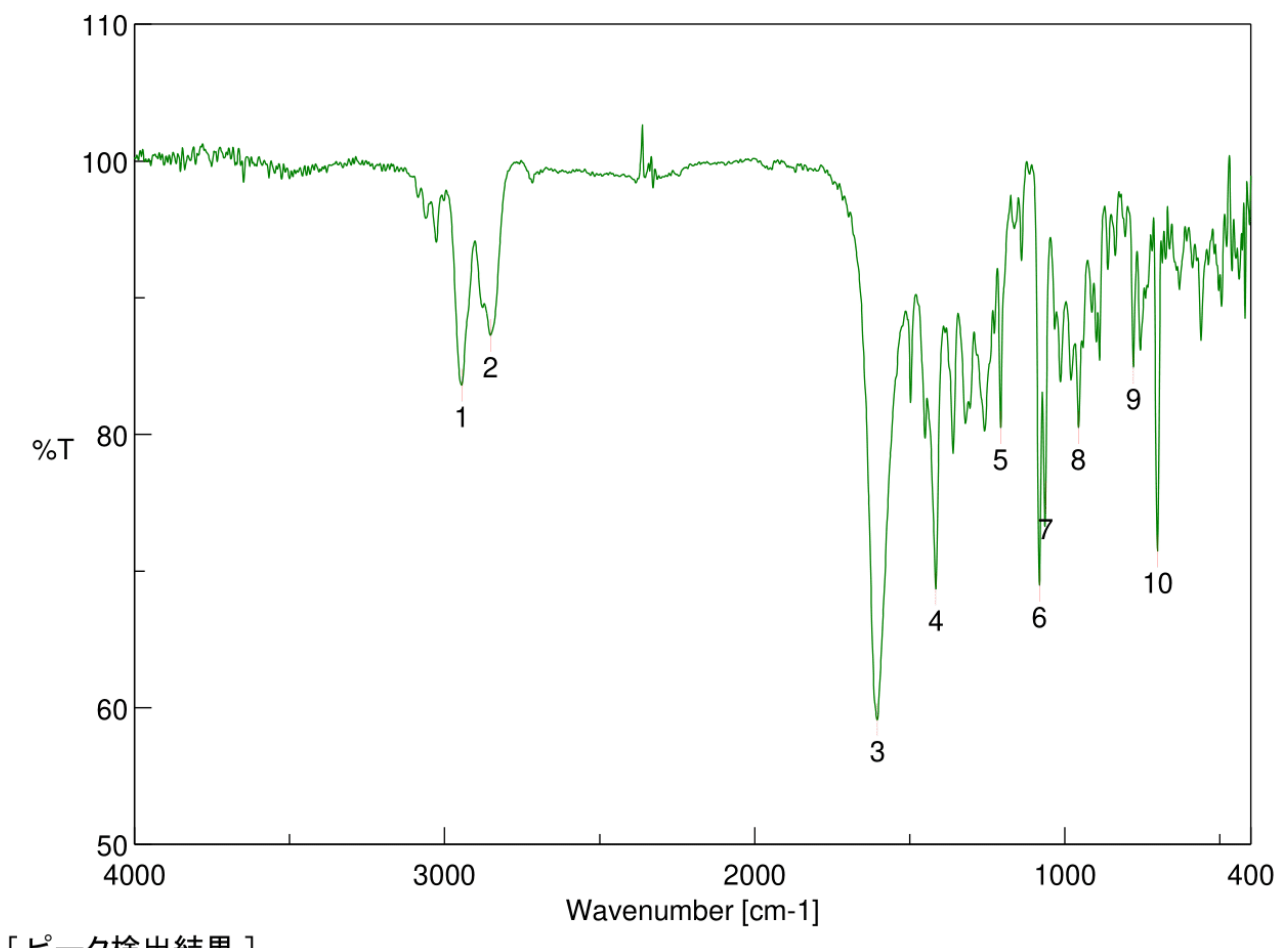

\begin{tabular}{|c|c|c|c|c|c|}
\hline \multicolumn{6}{|c|}{ [ピーク検出結果 ] } \\
\hline No. & 位置 & 強度 & No. & 位置 & 強度 \\
\hline 1 & 2944.77 & 83.5763 & 2 & 2852.2 & 87.2318 \\
\hline 3 & 1604.48 & 59.1021 & 4 & 1416.46 & 68.6795 \\
\hline 5 & 1207.22 & 80.4845 & 6 & 1081.87 & 68.9415 \\
\hline 7 & 1061.62 & 75.3872 & 8 & 955.555 & 80.4876 \\
\hline & 779.101 & 84.8967 & 10 & 700.998 & 4453 \\
\hline
\end{tabular}

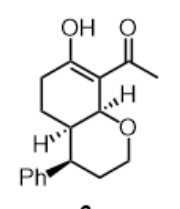

$6 a$ 


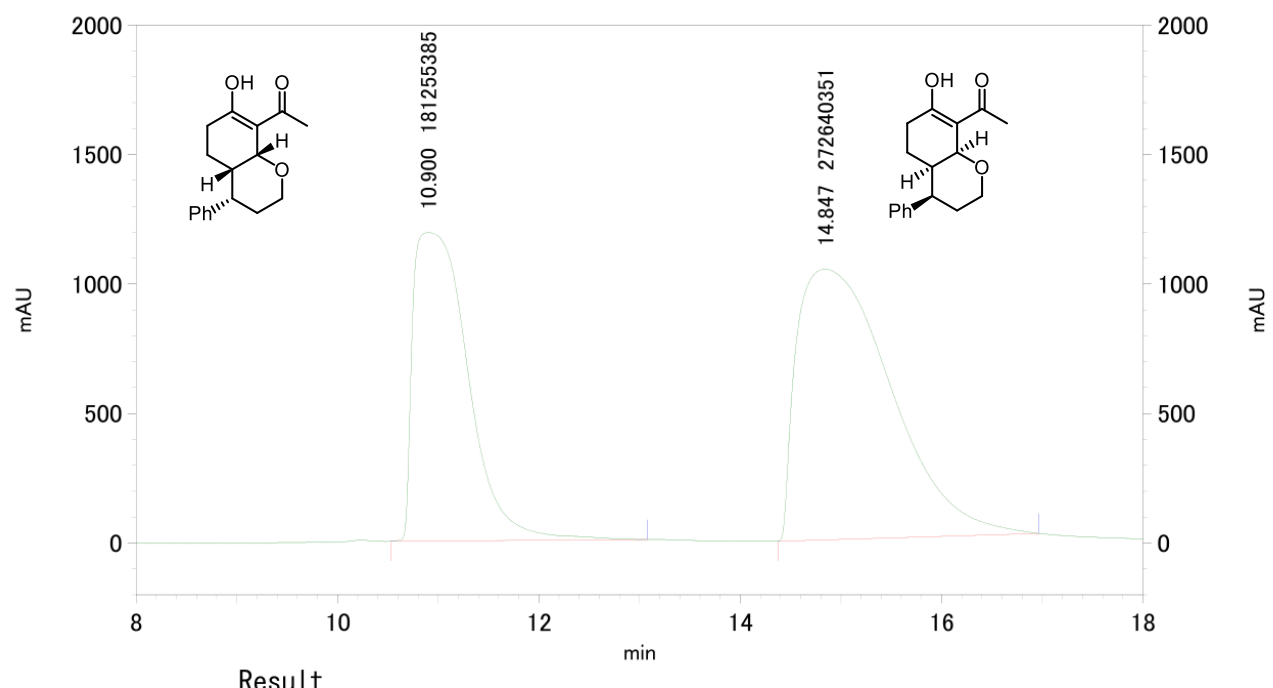

1: $210 \mathrm{~nm}, 4 \mathrm{~nm}$ 結果

Pk \# Retention time / min

Integration/ \%

39.933

Total

100.000 


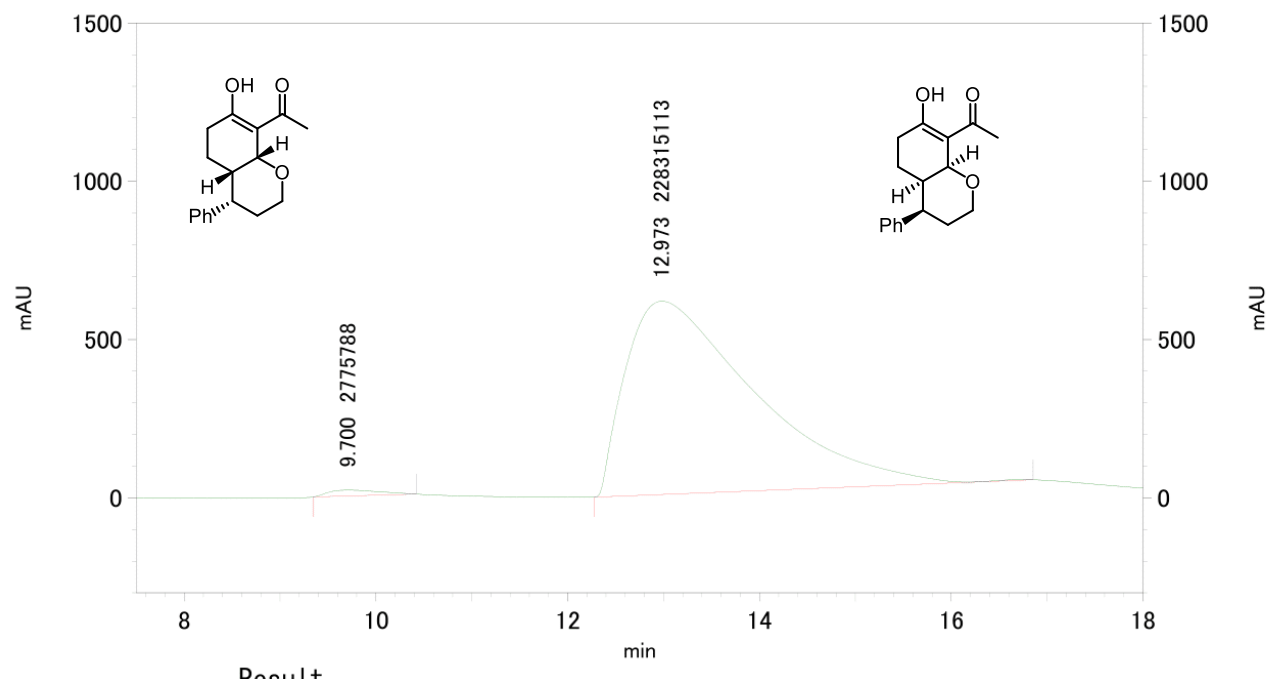

1: $210 \mathrm{~nm}, 4$ nm結果

Pk \# Retention time / min

Integration/\%

9. 700

98. 799

\begin{tabular}{|r|r|r|}
\hline$r-$ 㚈 & 100.000 \\
\hline
\end{tabular} 
10E.

6เฉ:।

$902 t$

tzzt

09275

$\varepsilon \angle 6^{\circ} 9$
$110^{\circ}$

$\varepsilon \angle 8^{\circ} 9=$

$+68^{\circ} \cdot-$
$982^{\circ} L$

$\left.98 Z^{\prime} t\right]$

$96 Z^{\circ} L$



$0 I \varepsilon: t]$

$\left.81 \varepsilon^{\circ} L\right]$

दृत.

पह

$6 \varepsilon^{\circ}$

$\mathrm{s} \varepsilon^{\circ}$

$89 \varepsilon^{\circ} L$

$1 / \varepsilon \cdot$

$9<\varepsilon \cdot$

6LE

$91 t^{\circ} \mathrm{L}$

दat

$\angle E D L$

$\mathrm{Z} t \mathrm{D}^{\circ} \mathrm{L}$

$8 b t^{\circ} L$

$G 9 b^{\circ} L-$

$9 \mathrm{St}^{\circ} L$

$69 b^{\circ} t$

$t L t: L$

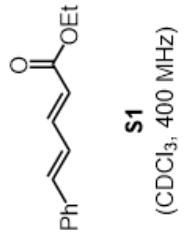

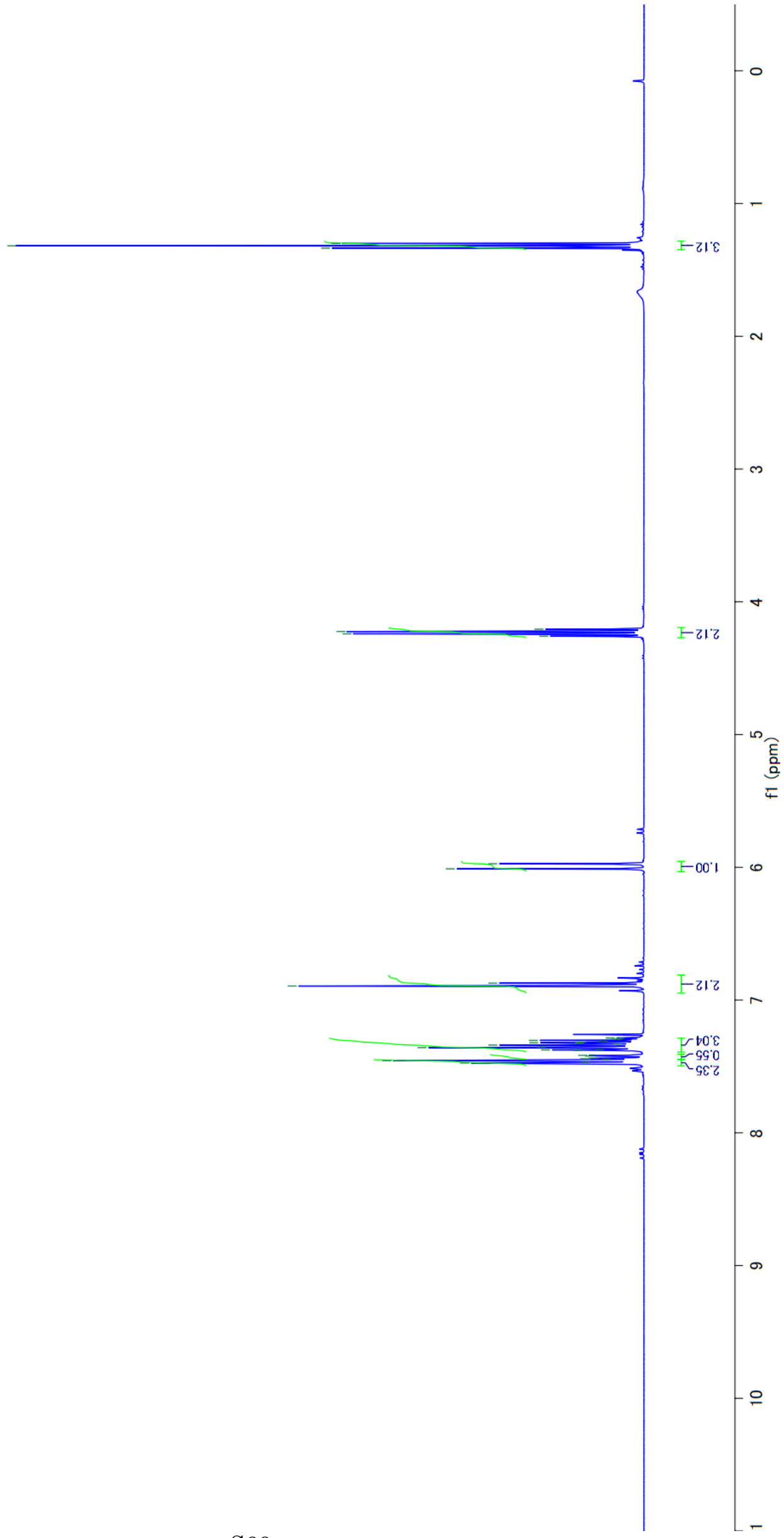




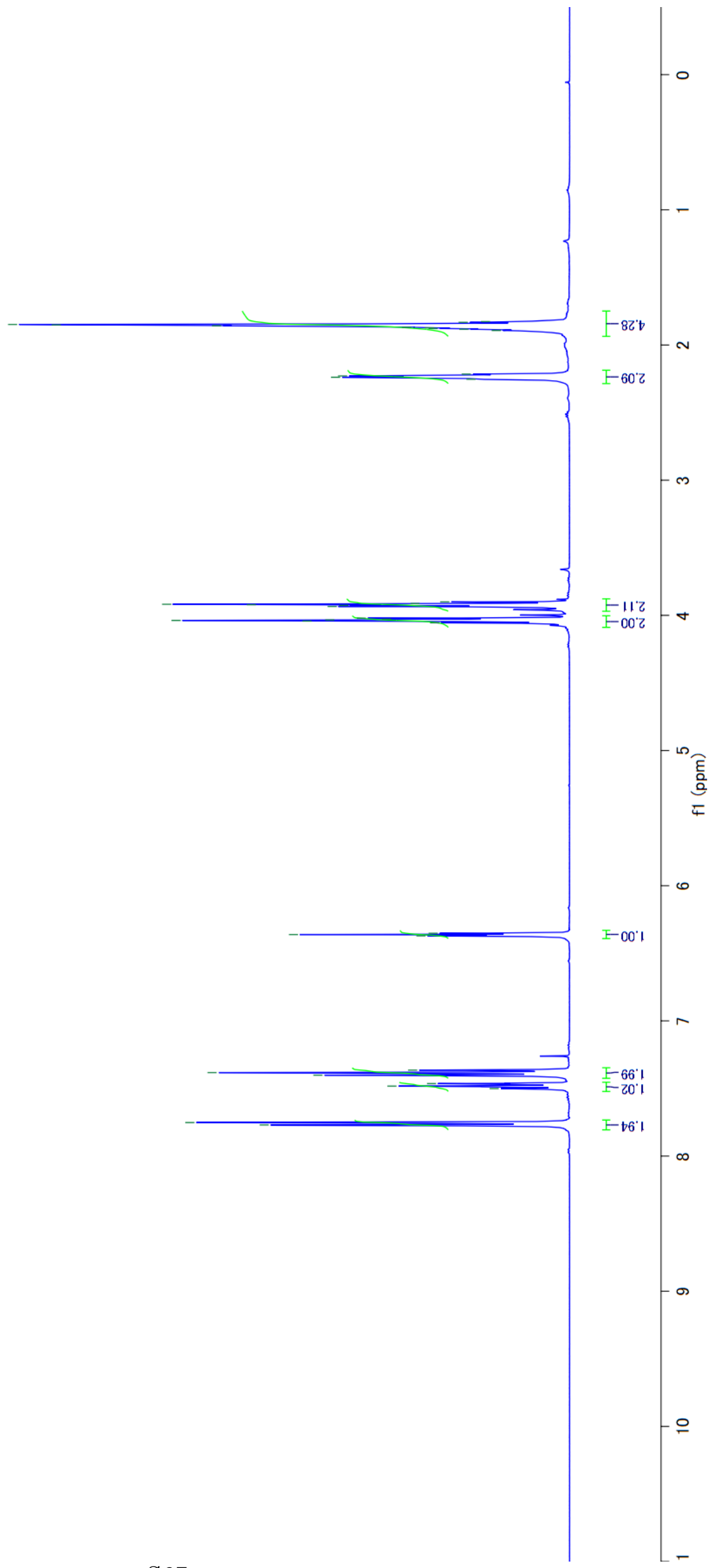




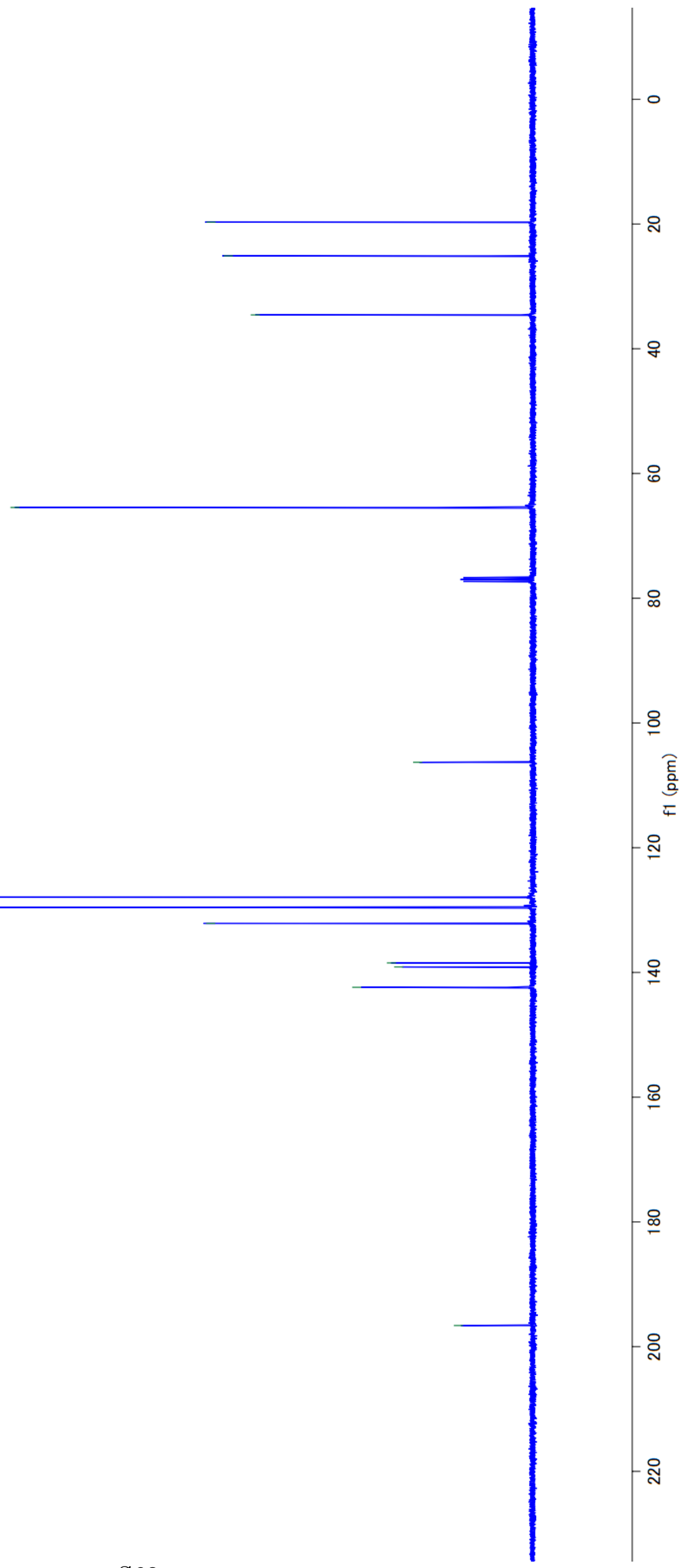

밀 


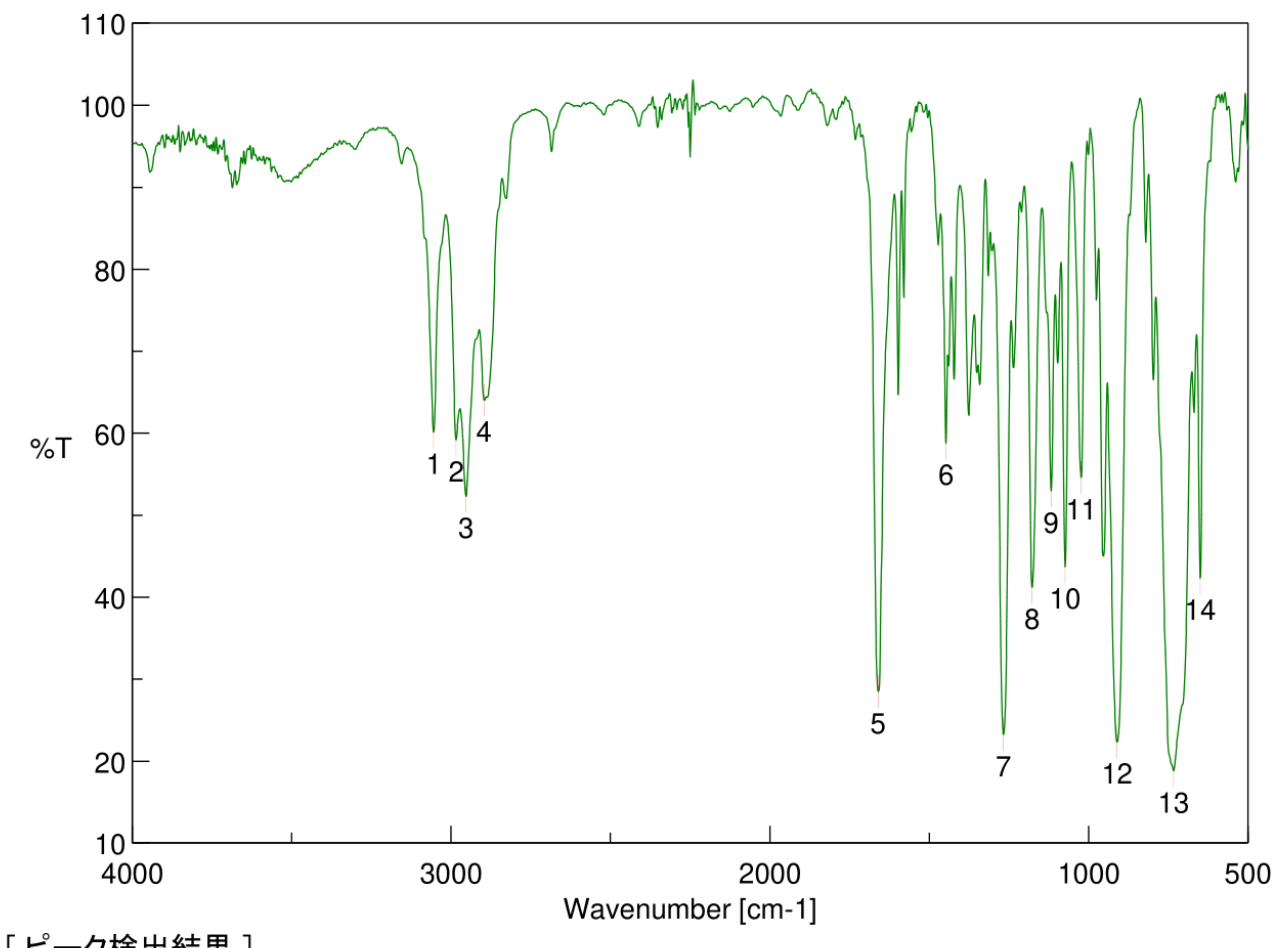

[ピーク検出結果 ]

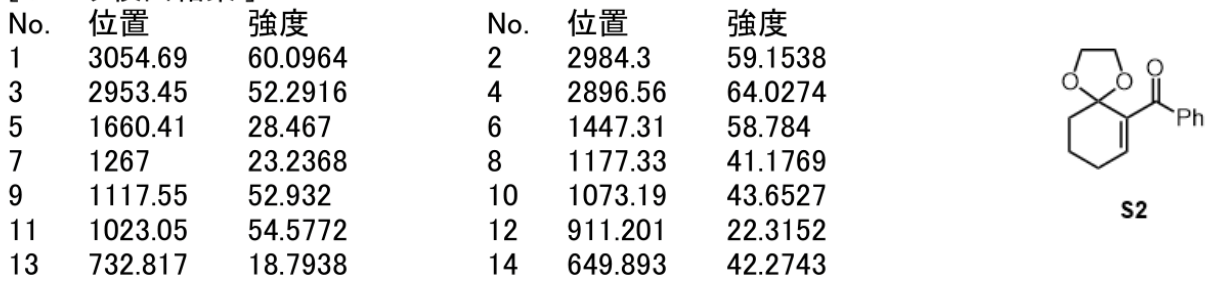




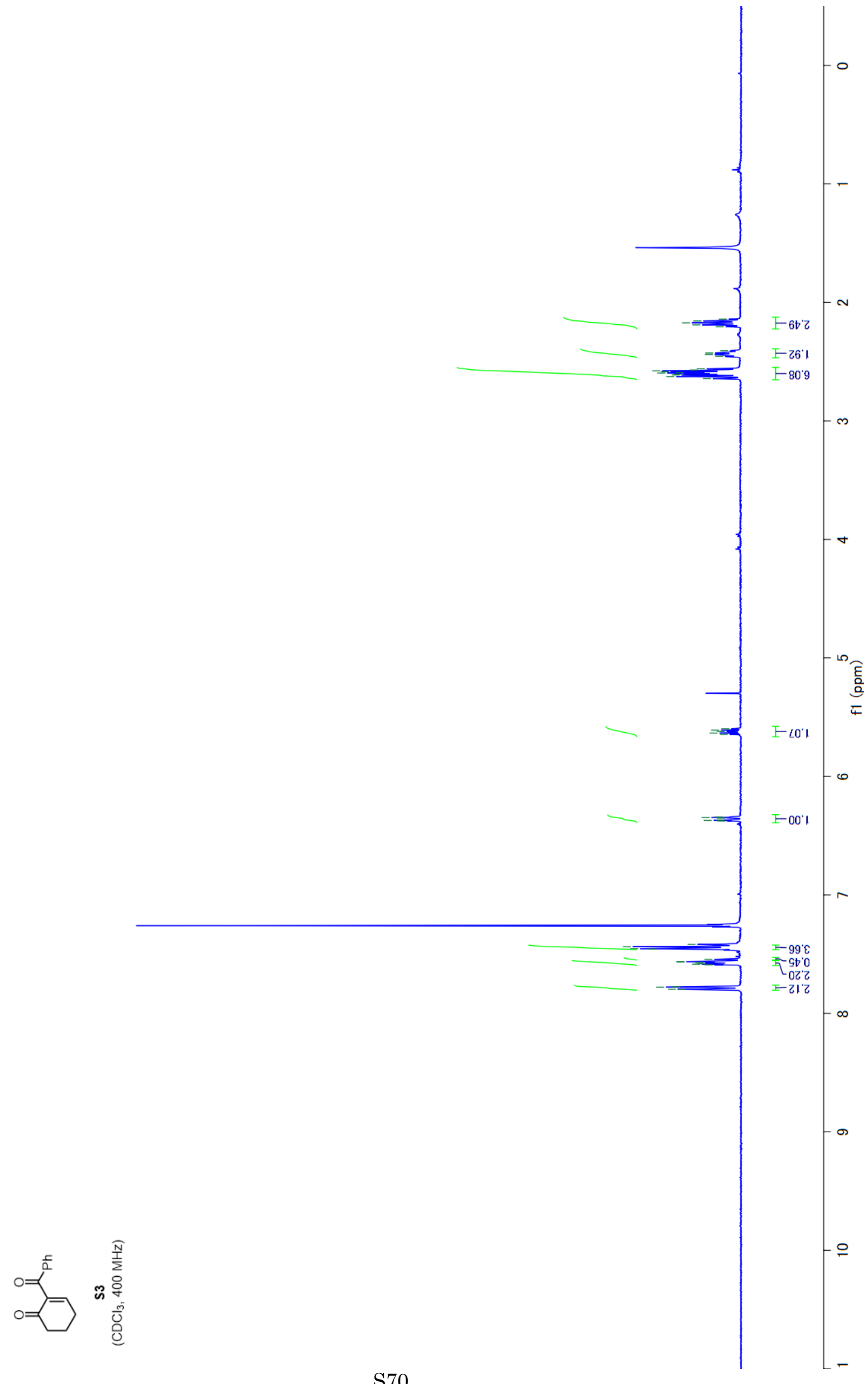



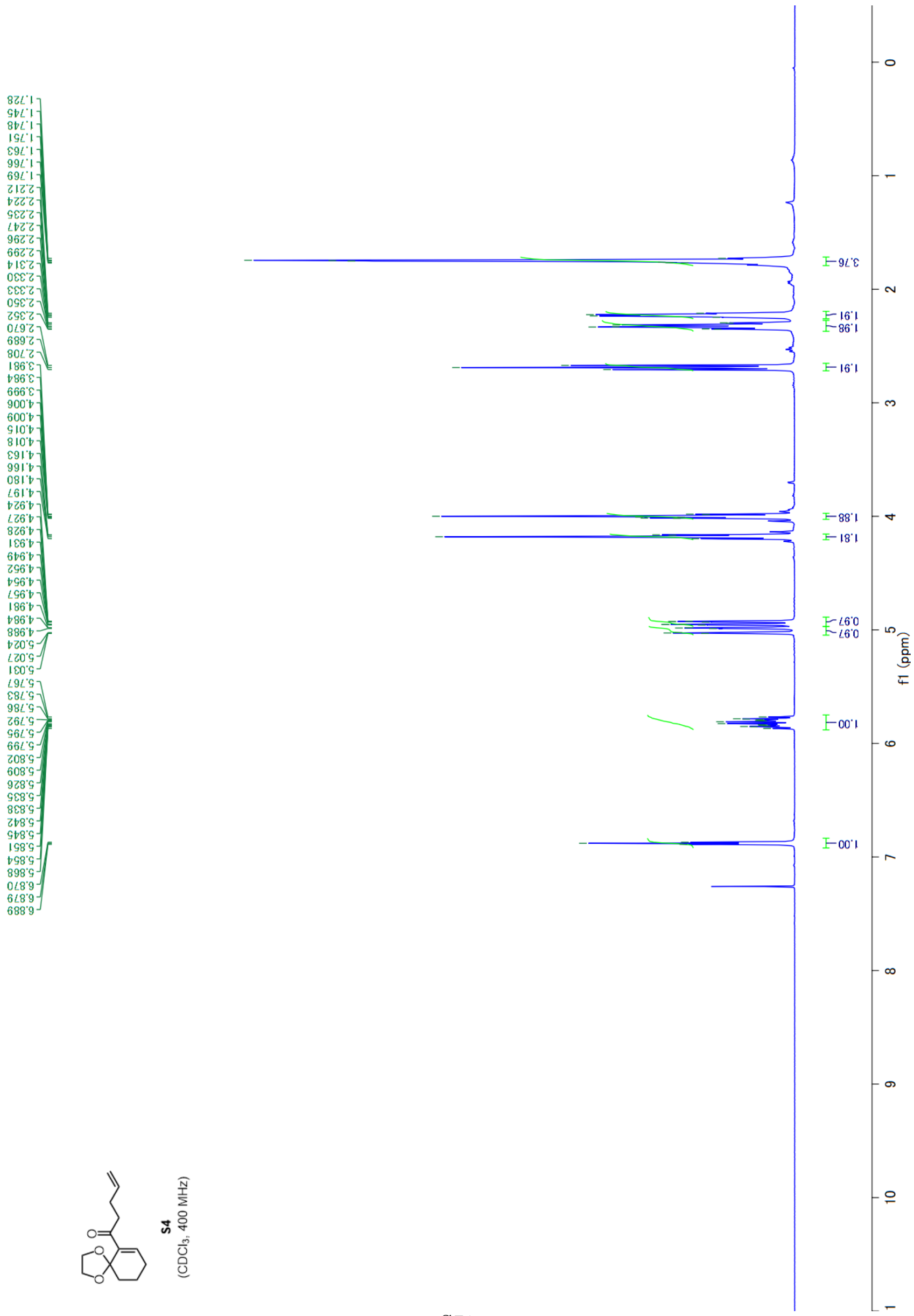


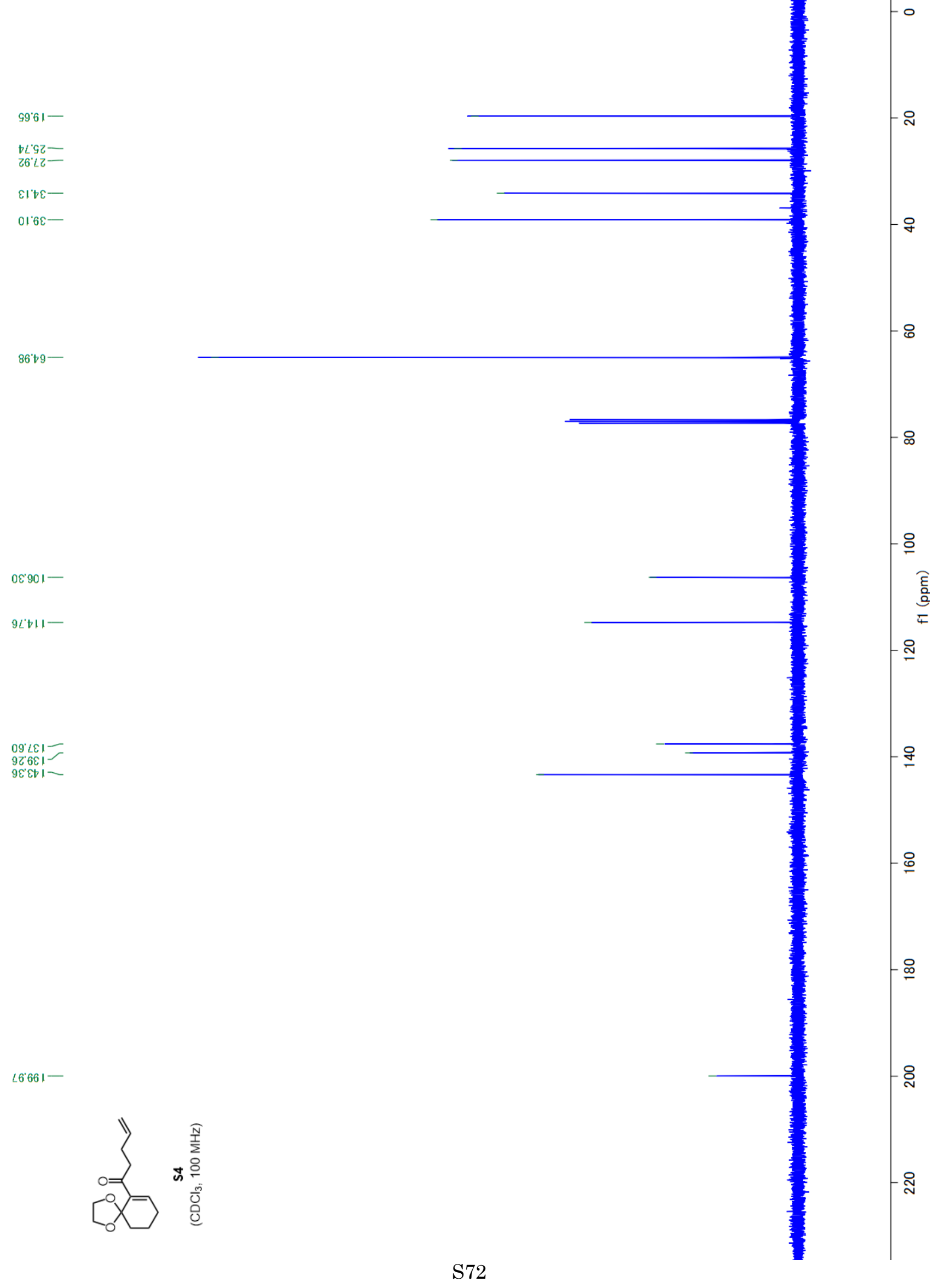




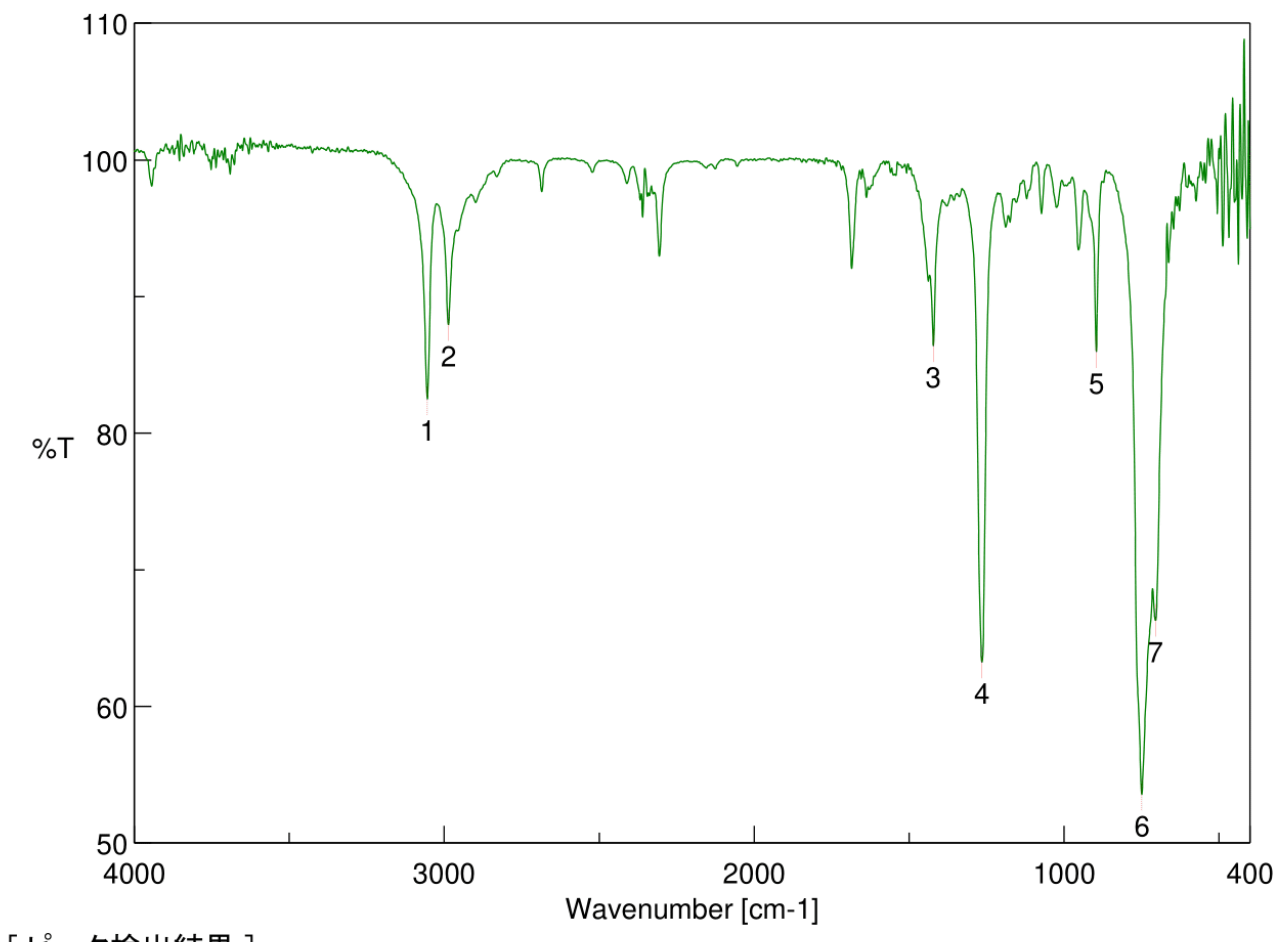

[ピーク検出結果 ]

$\begin{array}{llllll}\text { No. } & \text { 位置 } & \text { 強度 } & \text { No. } & \text { 位置 } & \text { 強度 } \\ 1 & 3054.69 & 82.4957 & 2 & 2986.23 & 87.9358 \\ 3 & 1421.28 & 86.3841 & 4 & 1265.07 & 63.2402 \\ 5 & 895.773 & 85.9551 & 6 & 749.209 & 53.5563\end{array}$

$704.855 \quad 66.2747$

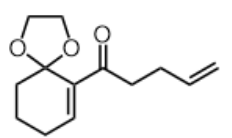

S4 


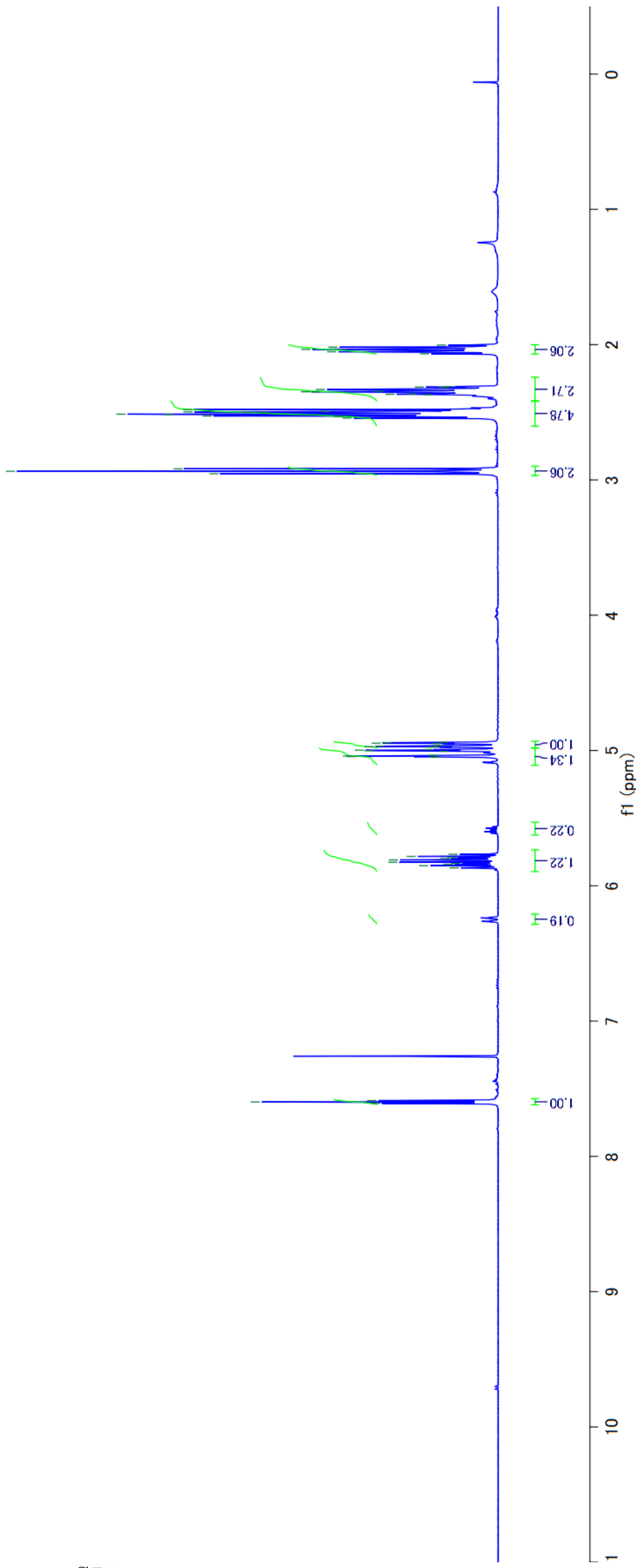




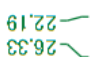

$18.42=$

$06.8 \varepsilon-$

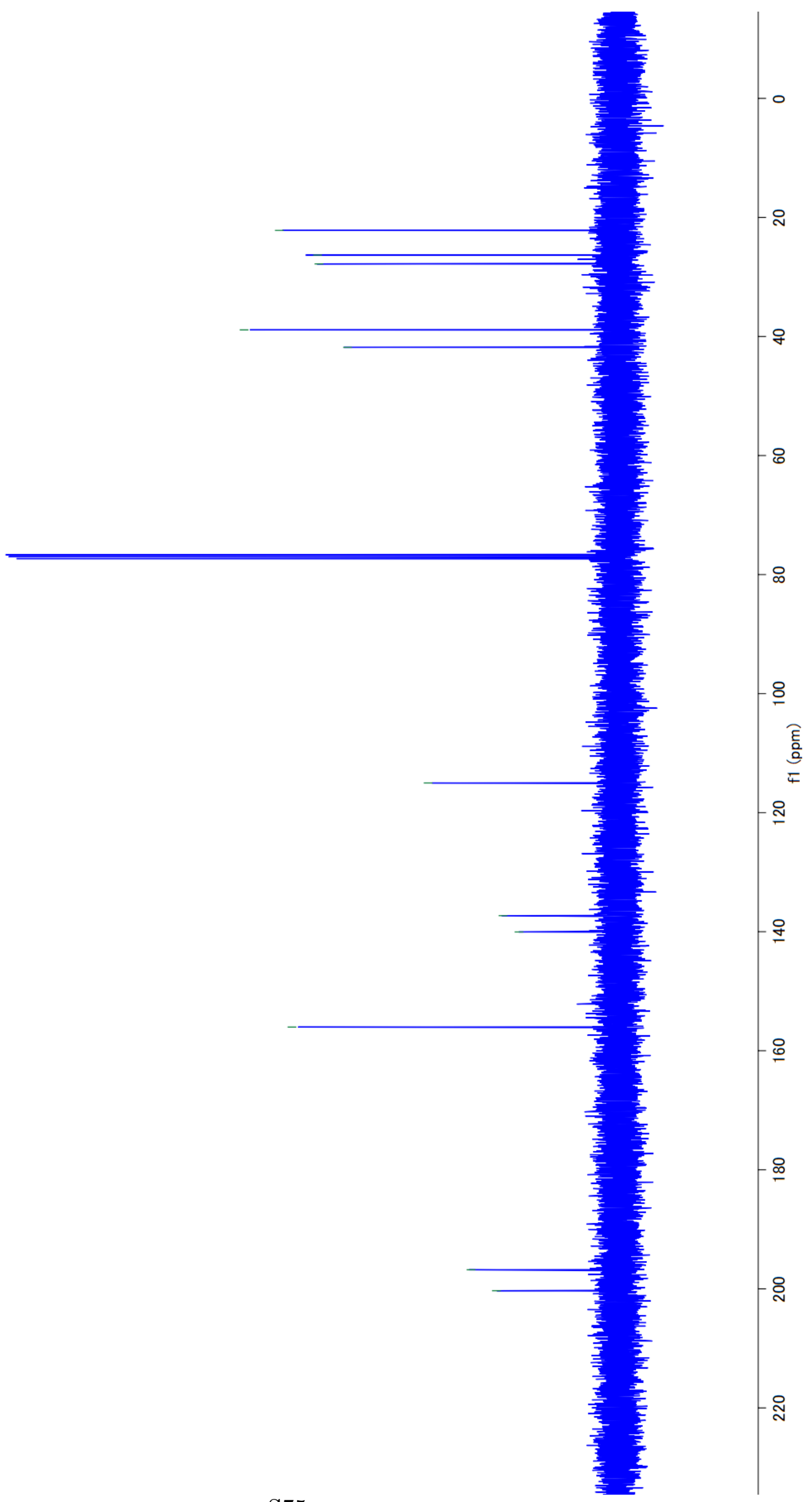

10'911-

$0 \varepsilon\llcorner\varepsilon \perp-$

to 001 -

$20.991-$

6L' $961-$

เE'00Z-

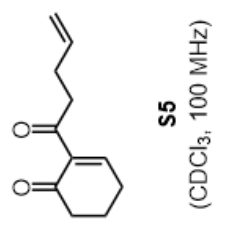




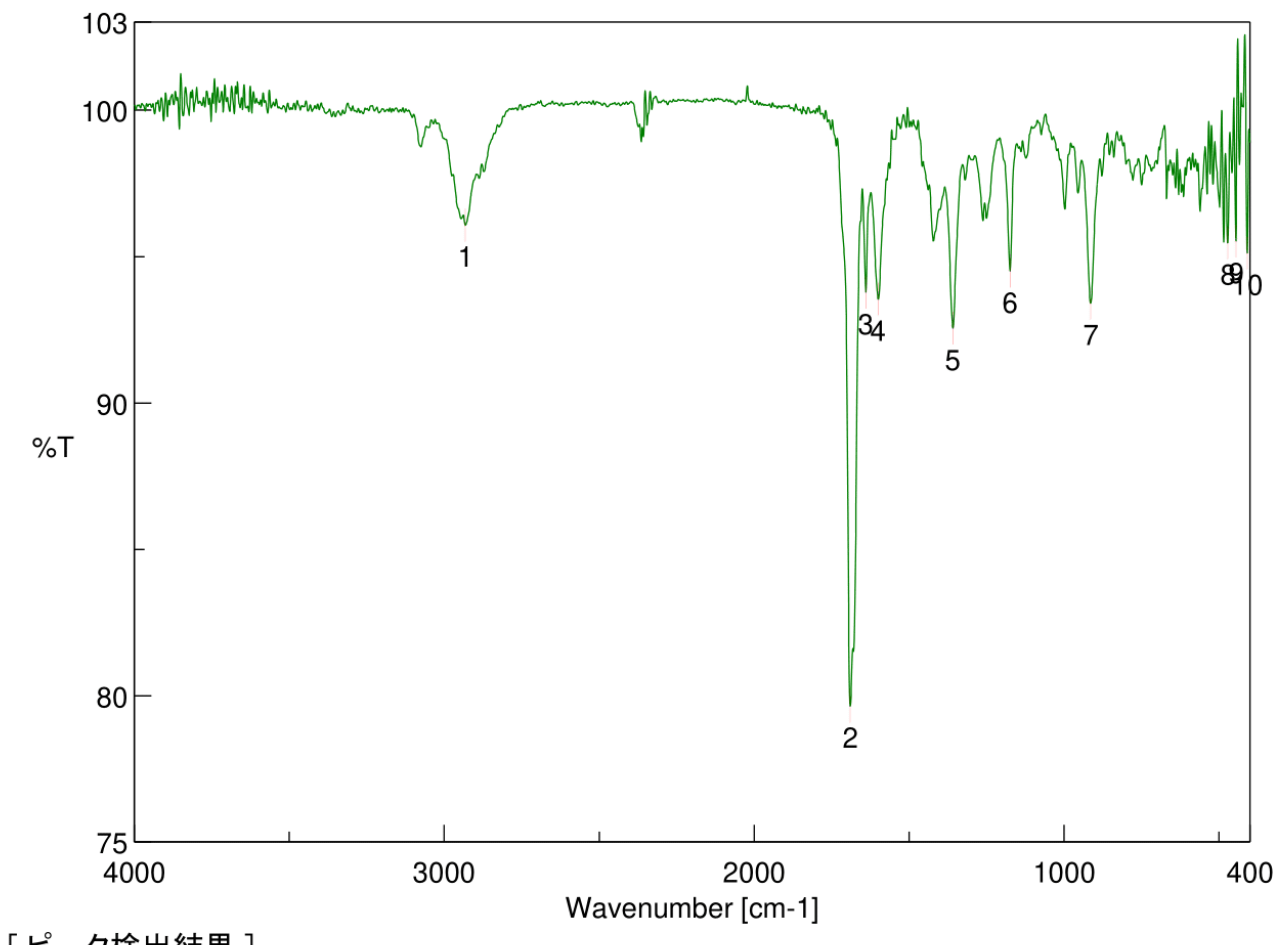

[ピーク検出結果 ]

$\begin{array}{lllllllll}\text { No. } & \text { 位置 } & \text { 強度 } & \text { No. } & \text { 位置 } & \text { 強度 } & & \\ 1 & 2932.23 & 96.0623 & 2 & 1690.3 & 79.6417 & \\ 3 & 1639.2 & 93.766 & 4 & 1599.66 & 93.5391 & & \\ 5 & 1358.6 & 92.5521 & 6 & 1174.44 & 94.4901 & & \\ 7 & 914.093 & 93.3953 & 8 & 471.51 & 95.4602 & & \\ 9 & 445.476 & 95.5242 & 10 & 408.835 & 95.1126 & \text { S5 }\end{array}$




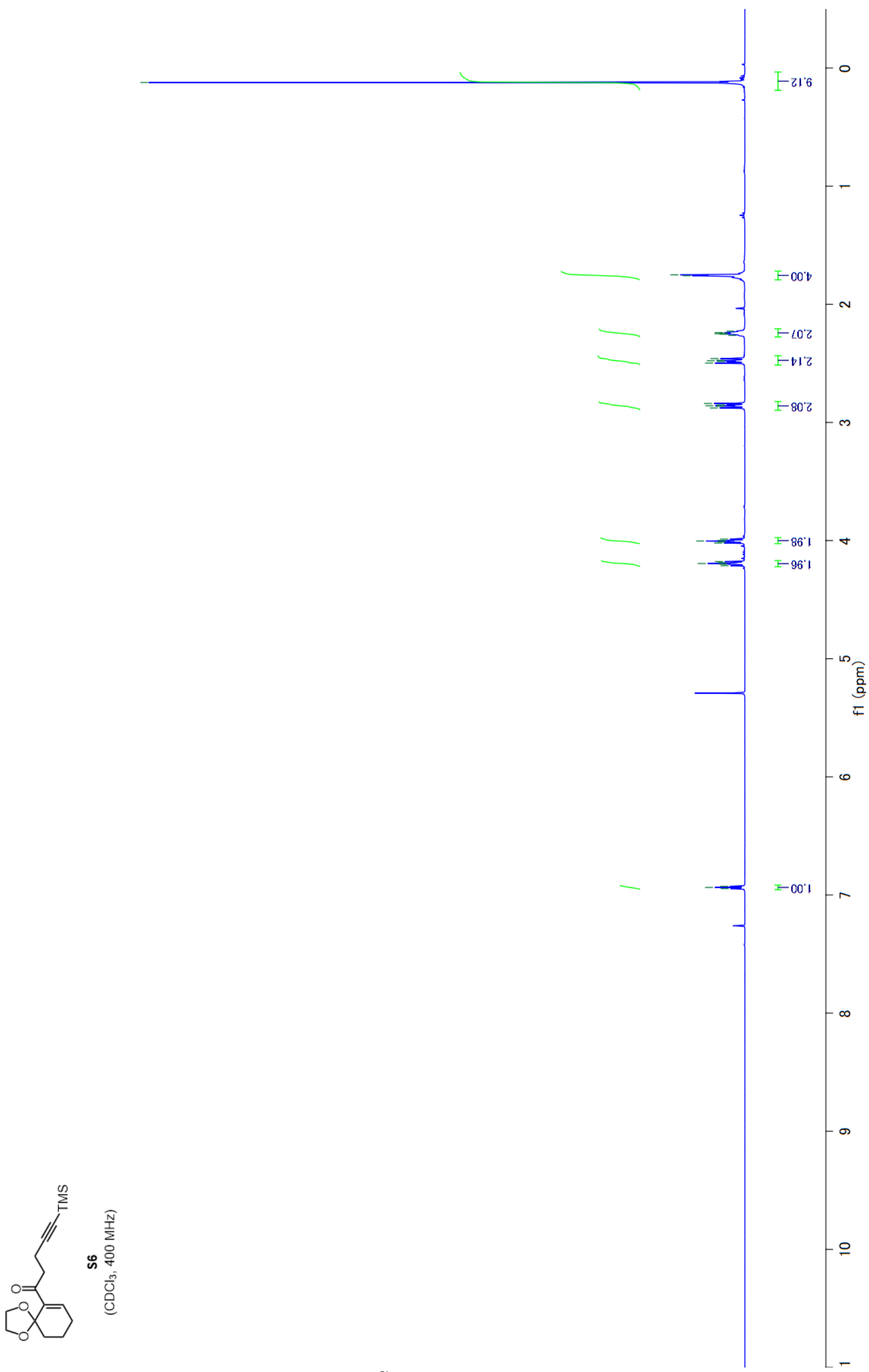


$40^{\circ}-$

$00^{\circ}+1-$

9961 -

乙8'९乙-

$12+5 \varepsilon$

$116 \varepsilon-$

so's9-

$8278-$

$\begin{aligned} & 61901 \\ & {\left[\varepsilon^{\prime} 901\right.}\end{aligned}>$

$88^{\circ} 81-$

$01501-$

61'861-<smiles>CCC#CCCC(=O)C1=CCCCC12OCCO2</smiles>

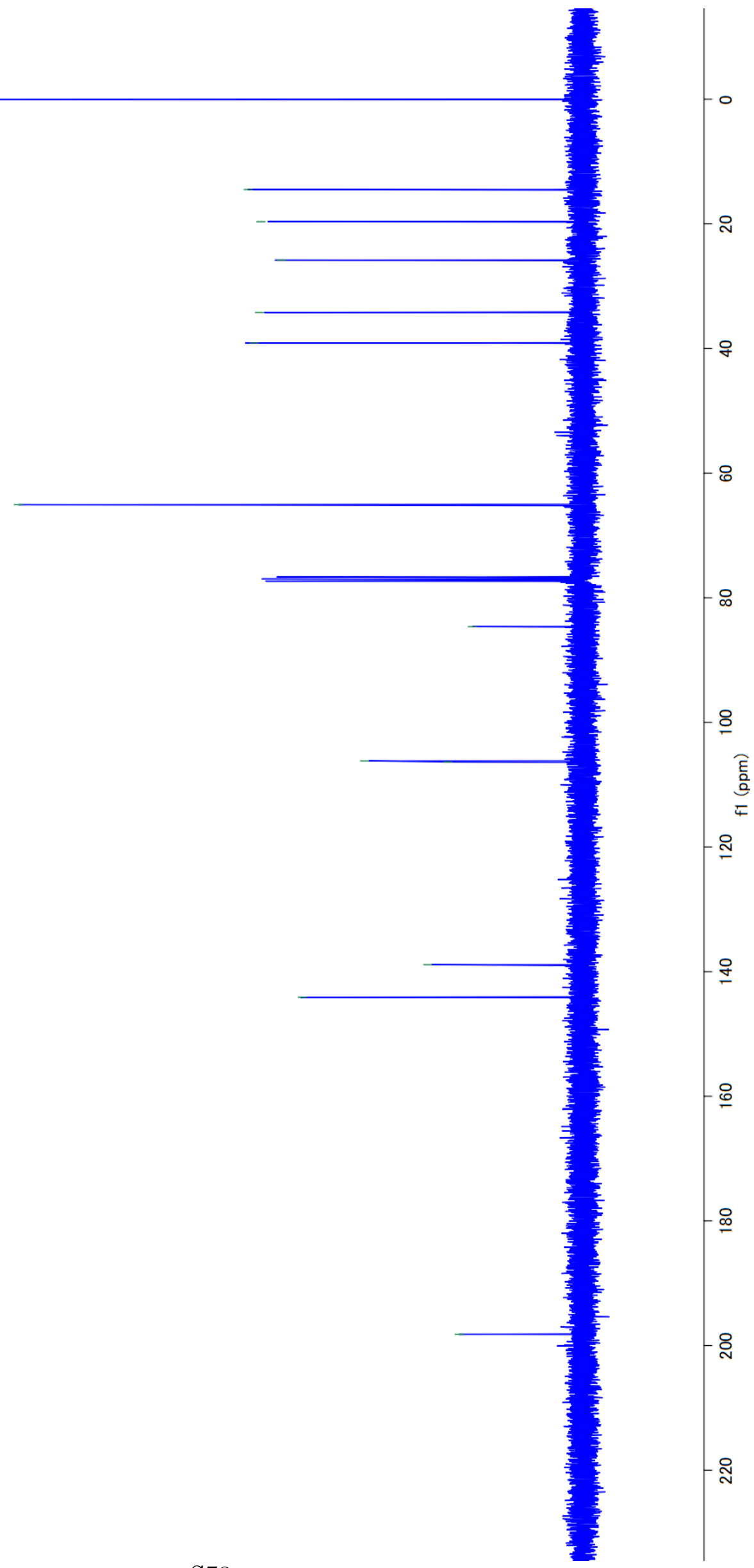




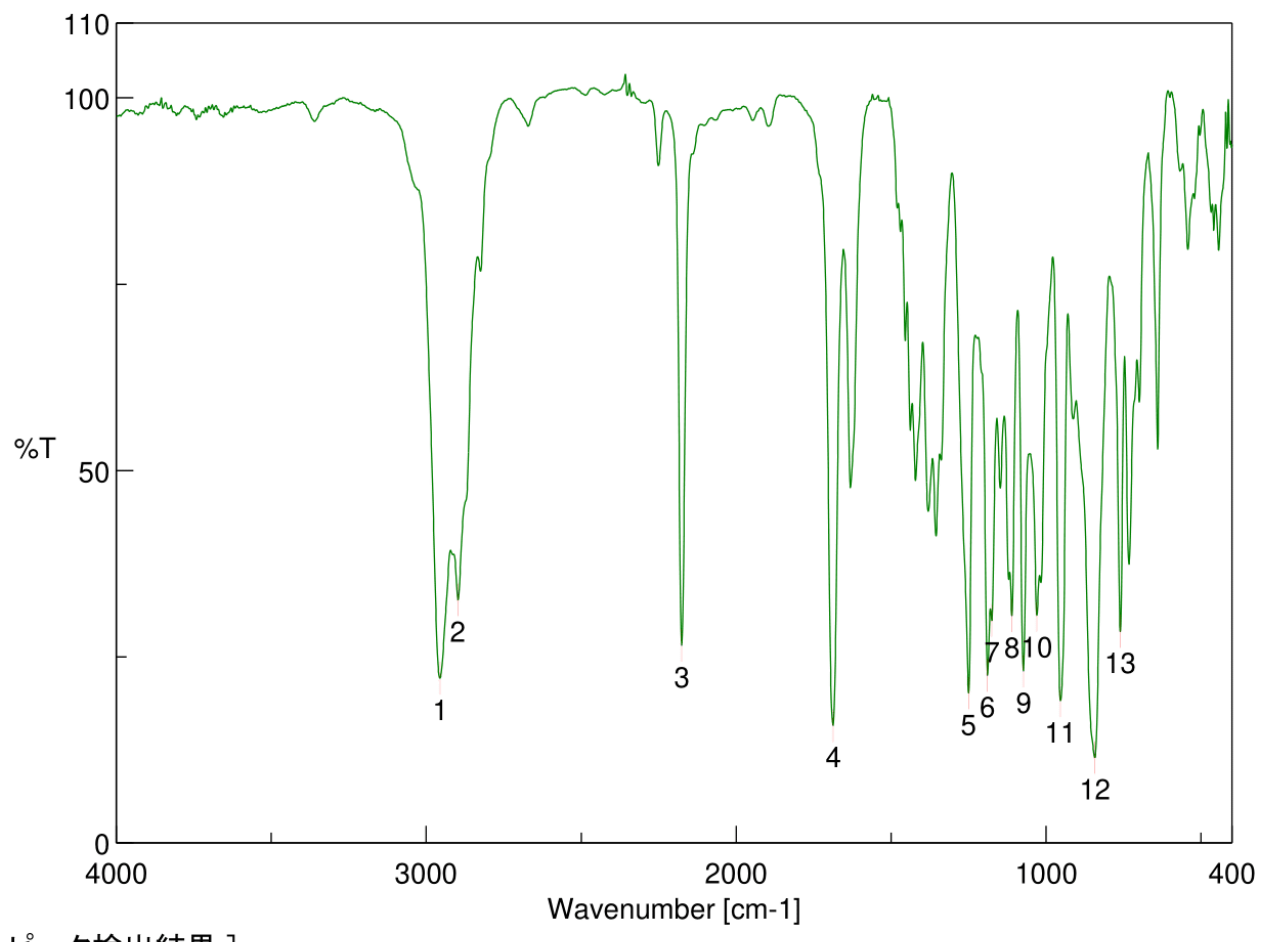

[ピーク検出結果 ]

No. 位置 強度 No. 位置 強度

$\begin{array}{llllll}1 & 2955.38 & 22.1161 & 2 & 2897.52 & 32.5794\end{array}$

$\begin{array}{llllll}3 & 2175.31 & 26.4994 & 4 & 1687.41 & 15.7538\end{array}$

$\begin{array}{llllll}5 & 1249.65 & 20.0942 & 6 & 1188.9 & 22.4976\end{array}$

$\begin{array}{llllll}7 & 1175.4 & 29.8342 & 8 & 1109.83 & 30.4975\end{array}$

$\begin{array}{llllll}9 & 1073.19 & 23.0923 & 10 & 1029.8 & 30.5402\end{array}$

$\begin{array}{llllll}11 & 953.627 & 19.0941 & 12 & 842.74 & 11.435\end{array}$

$\begin{array}{lll}13 & 760.78 \quad 28.3727\end{array}$

$12 \quad 842.74 \quad 11.435 \quad$ S6 

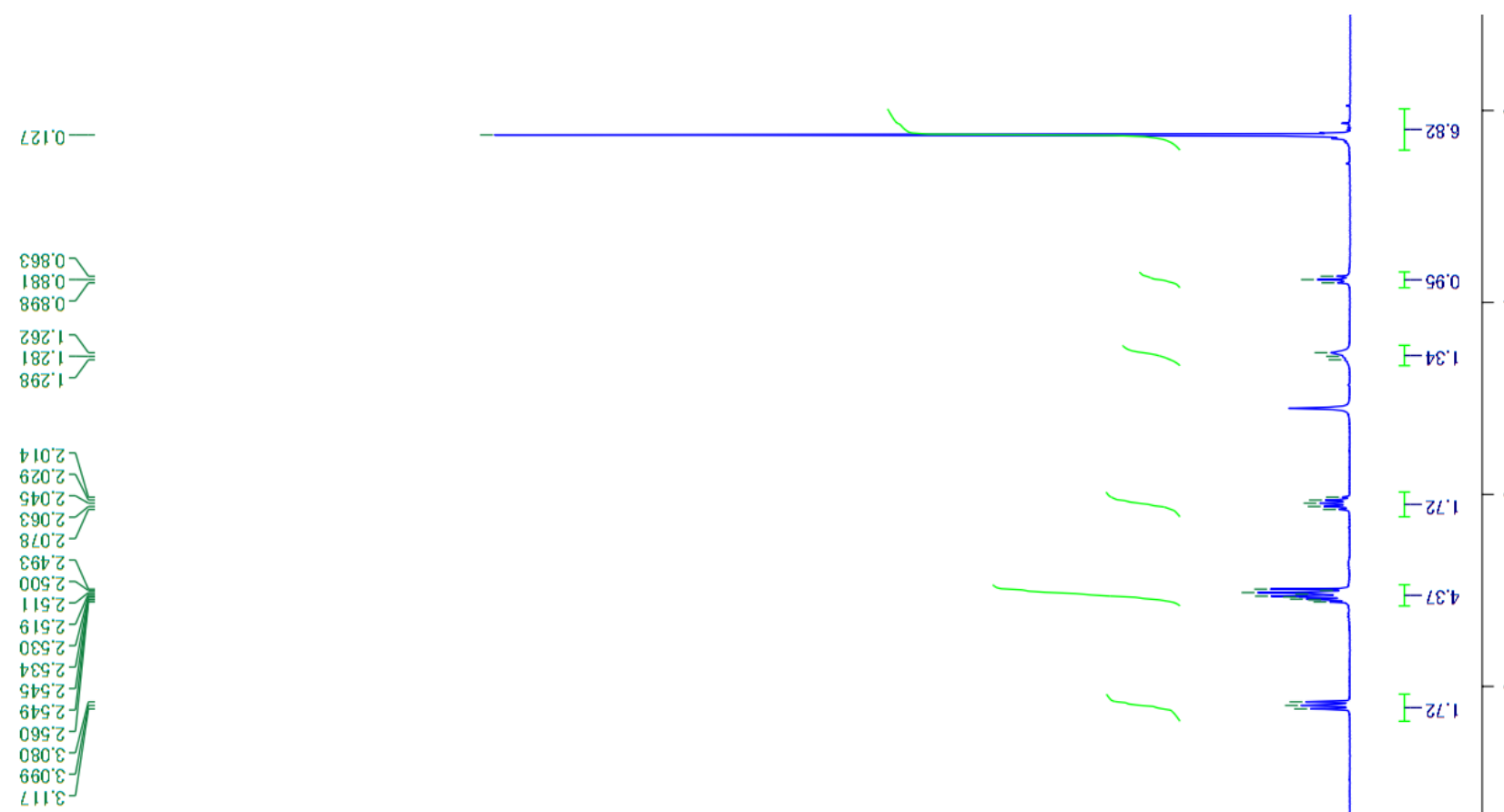

$88^{\circ}-$

$868^{\circ} 0$

18217

$10 \% 7$

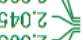

66t' 2

1197

$19 \mathrm{~g}$

बकs'

$b c^{\prime} \mathrm{z}$

$30^{\circ} \varepsilon$

LII

$0+9^{\circ} t>$

059 工

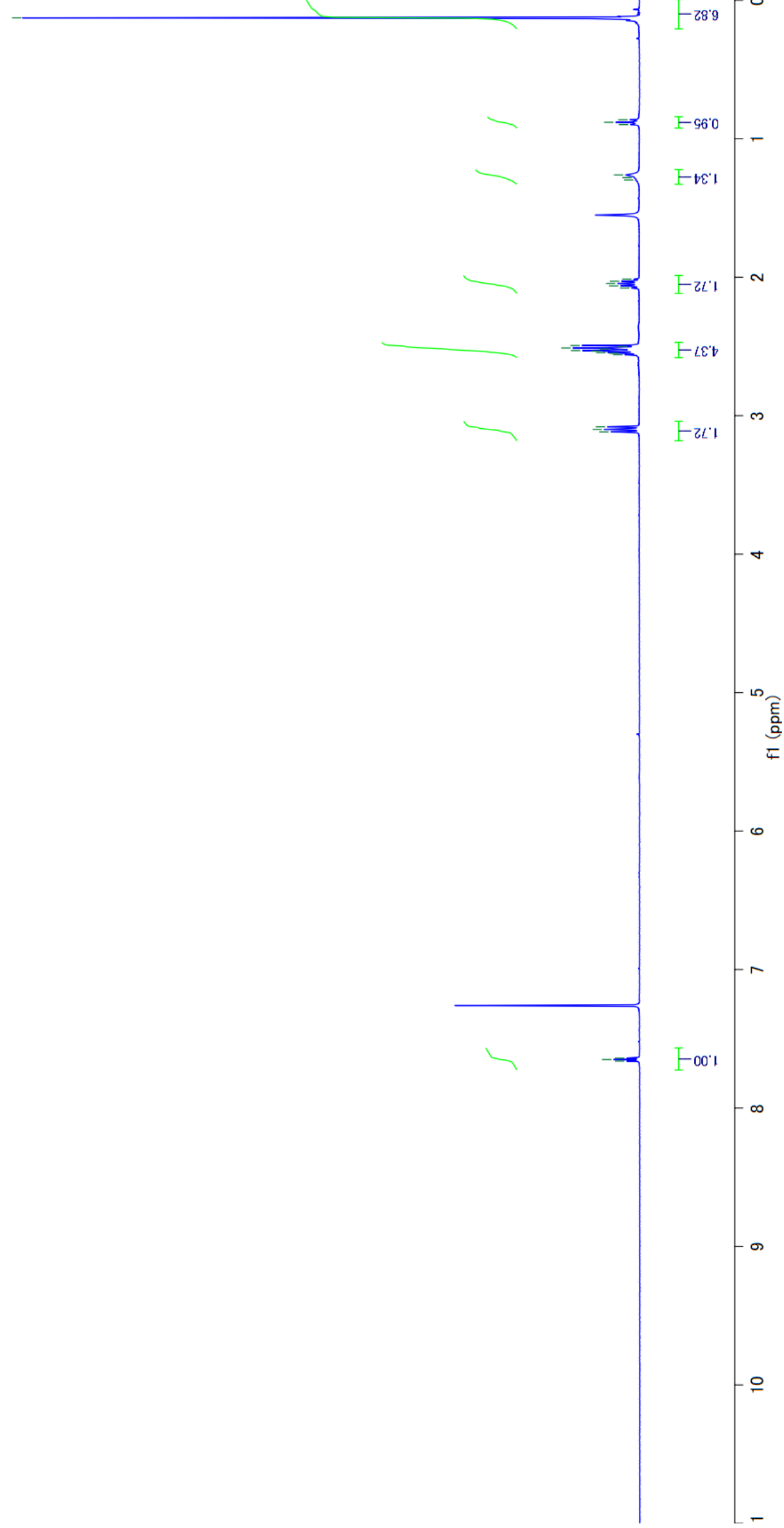


$90^{\circ} 0-$

$90+51-$

ยเ: -

เE.9Z-

98.8ะ-

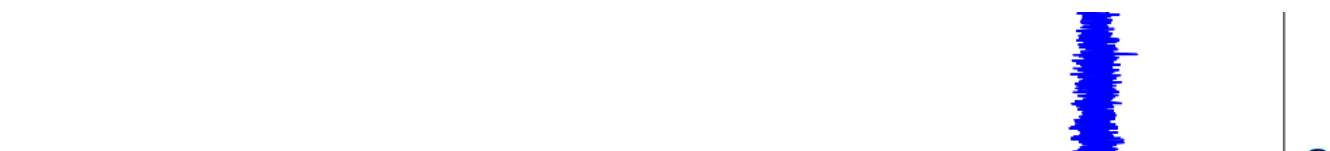

$89 \div 8-$

$96 \mathrm{GOI}-$

$09681-$

ะ9.9ต।-

$\varepsilon L 961=$
$19861=$

$\langle=$

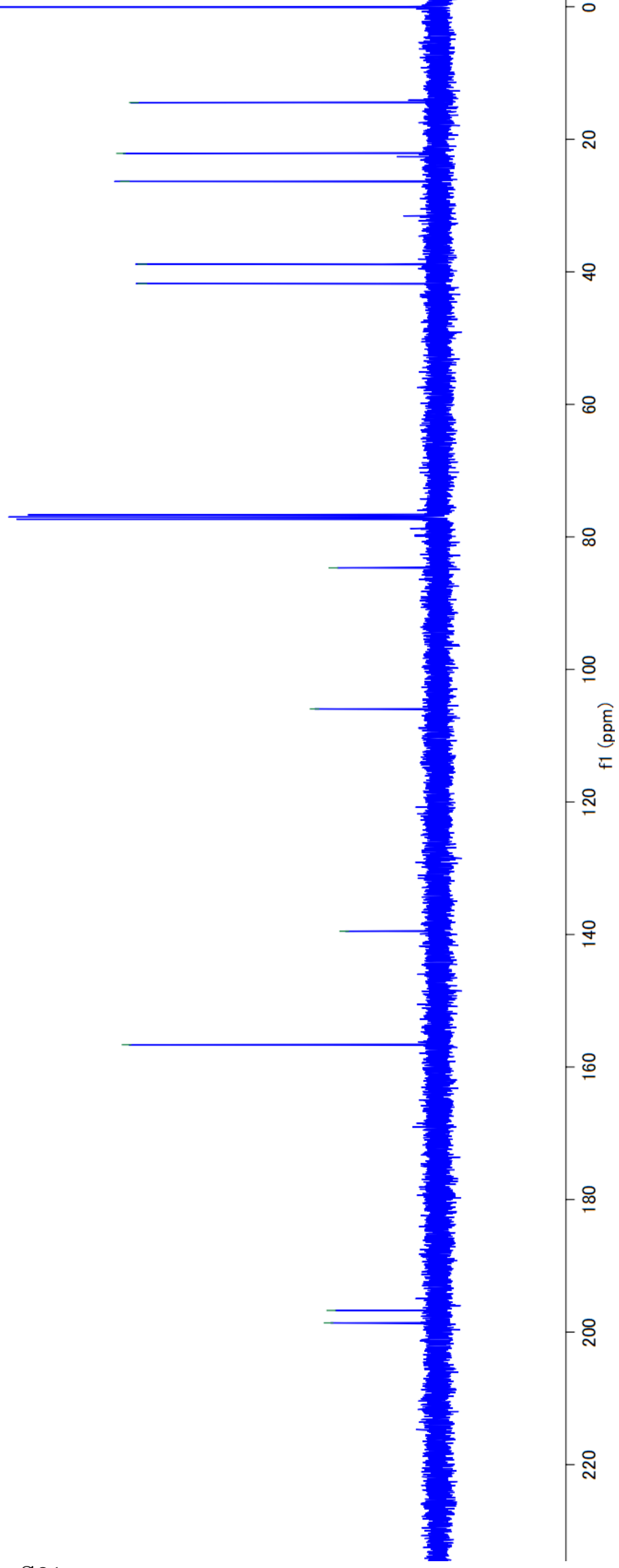




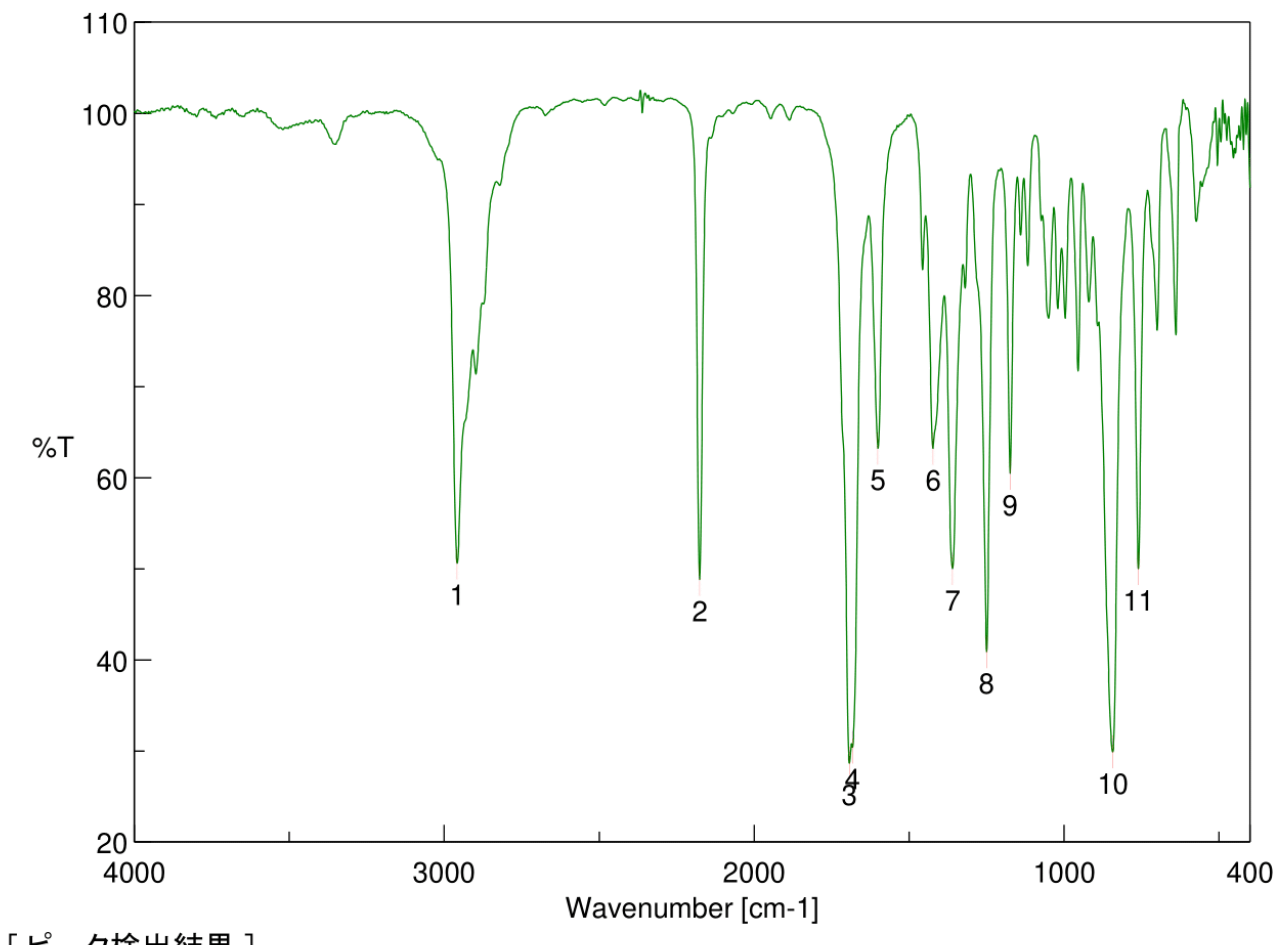

[ピーク検出結果 ]

\begin{tabular}{|c|c|c|c|c|c|c|}
\hline No. & 位置 & 強度 & No. & 位置 & 強度 & \\
\hline 1 & 2958.27 & 50.6065 & 2 & 2176.27 & 48.7929 & \\
\hline 3 & 1692.23 & 28.6337 & 4 & 1682.59 & 30.3891 & \\
\hline 5 & 1600.63 & 63.2086 & 6 & 1423.21 & 63.18 & \\
\hline 7 & 1359.57 & 49.9907 & 8 & 1249.65 & 40.8618 & \\
\hline 9 & 1174.44 & 60.4674 & 10 & 842.74 & 29.8832 & S7 \\
\hline 11 & 759.816 & 50.0004 & & & & \\
\hline
\end{tabular}




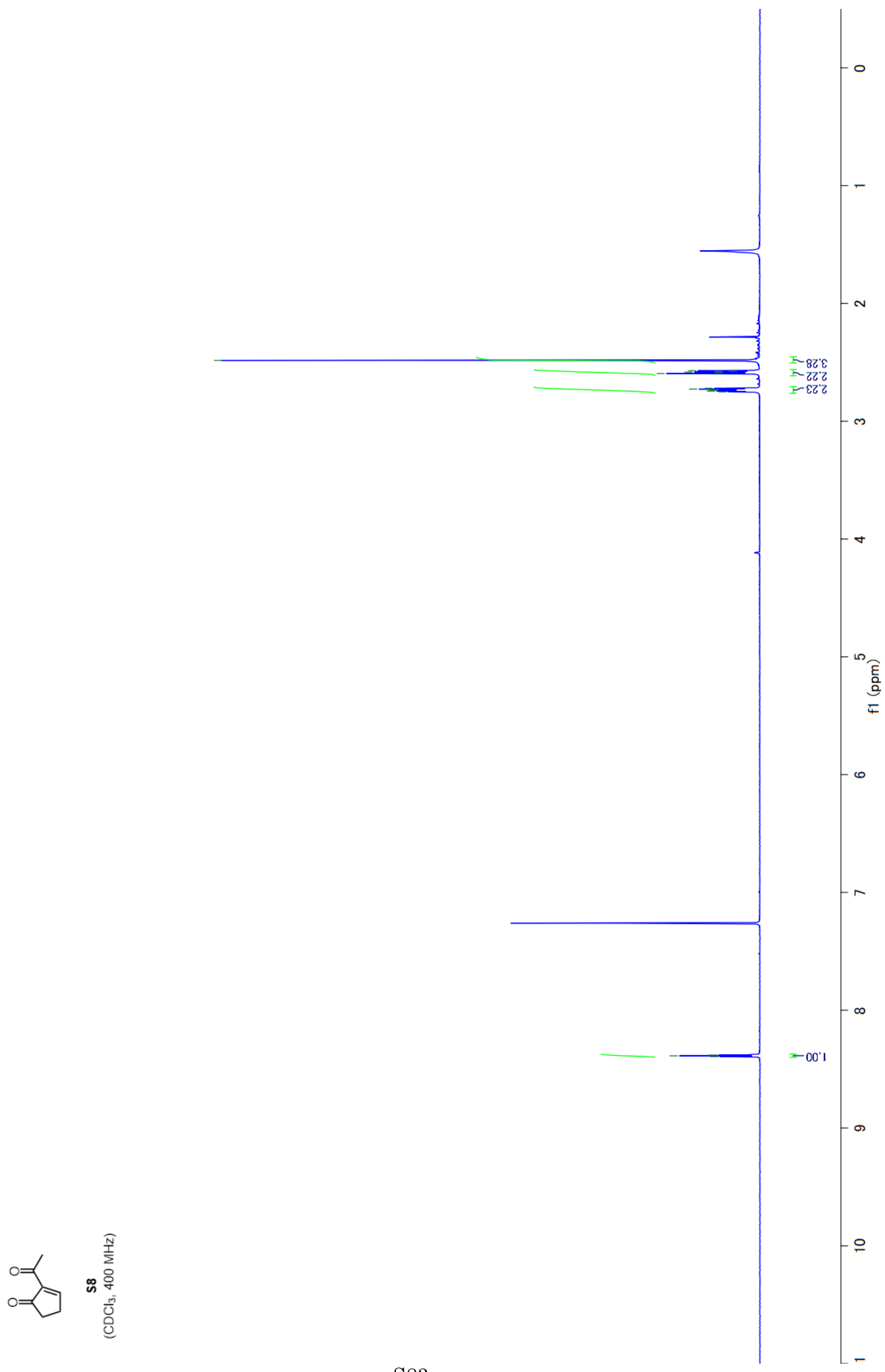



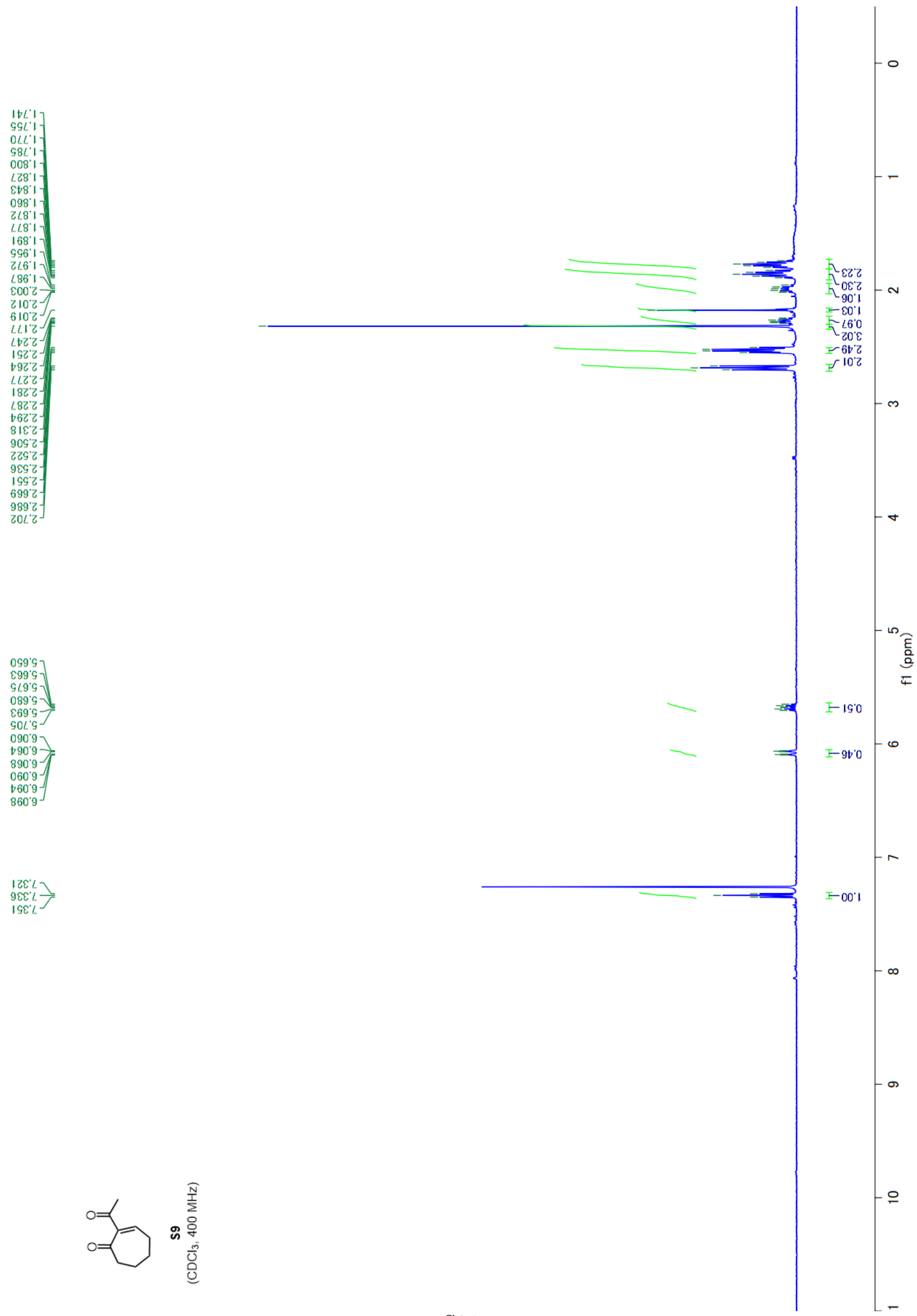


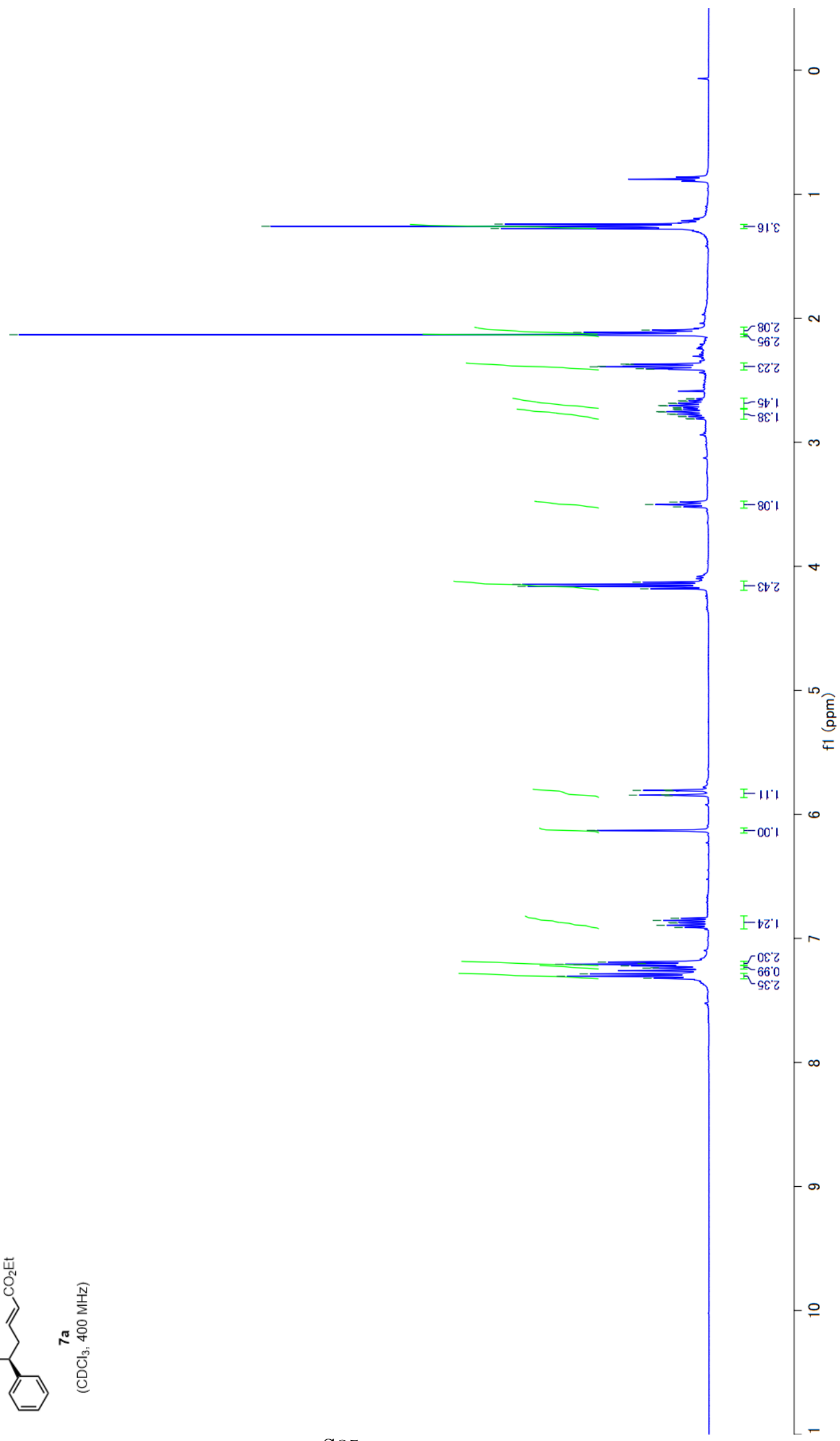




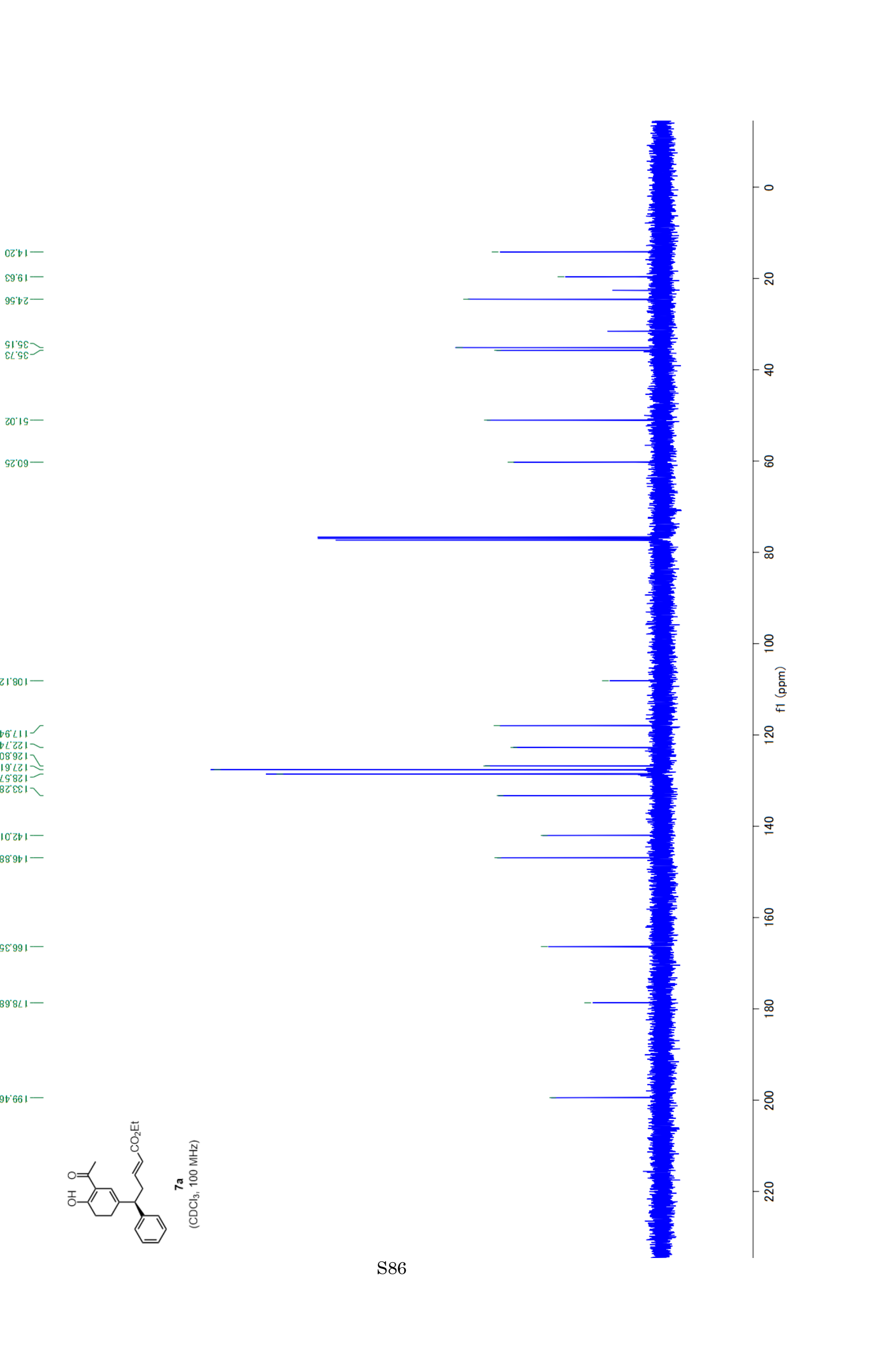




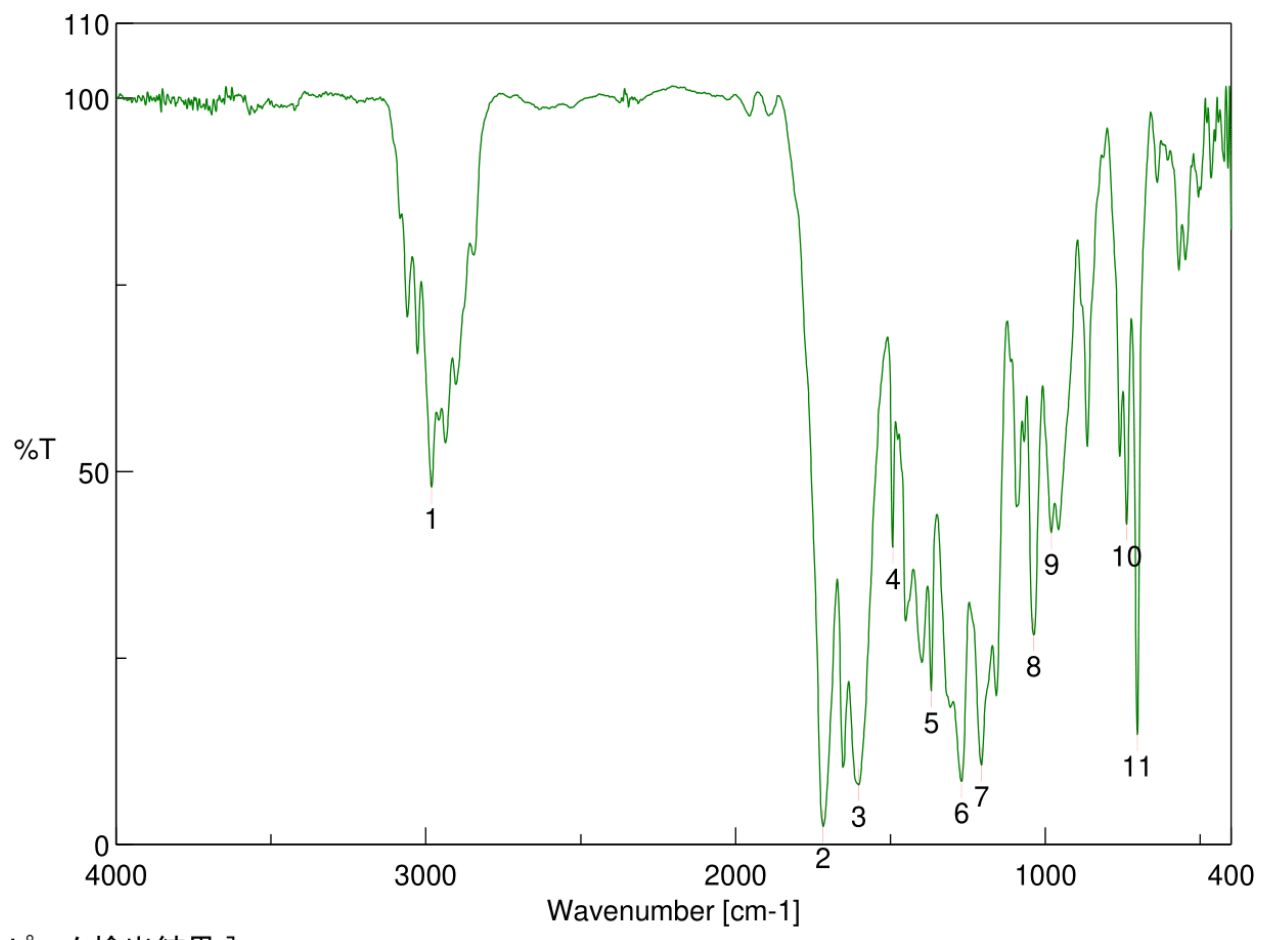

\begin{tabular}{lll}
\multicolumn{3}{l}{ [ ピーク検出結果 $]$} \\
No. & 位置 & 強度 \\
1 & 2981.41 & 47.8803 \\
3 & 1602.56 & 7.99203 \\
5 & 1368.25 & 20.582 \\
7 & 1206.26 & 10.644 \\
9 & 980.625 & 41.8158 \\
11 & 702.926 & 14.7508
\end{tabular}

No. 位置強度

$2 \quad 1717.3 \quad 2.41894$

$\begin{array}{lll}4 & 1492.63 & 39.8409\end{array}$

$\begin{array}{lll}6 & 1270.86 & 8.45216\end{array}$

$\begin{array}{lll}8 & 1037.52 & 28.1031\end{array}$

$\begin{array}{lll}10 & 737.639 & 42.87\end{array}$

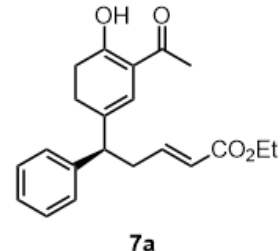




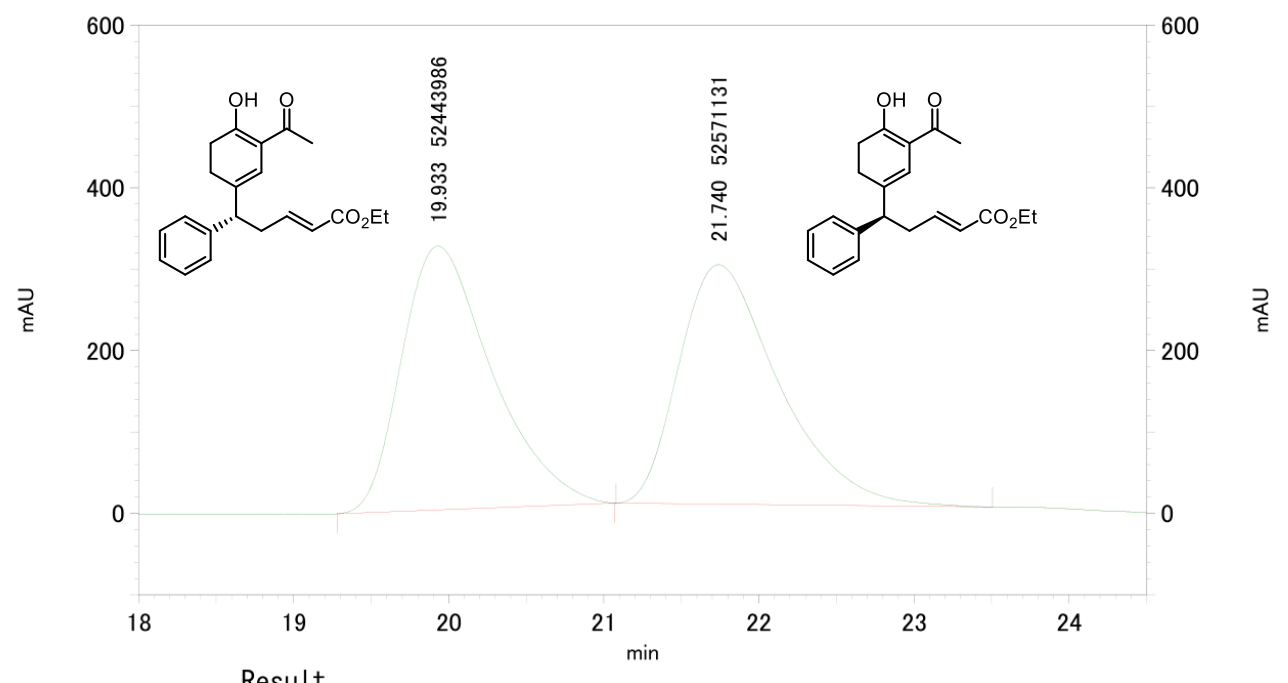

1: $210 \mathrm{~nm}, 4$ nm結果

Pk \# Retention time / min

Integration/\%

19. 933

50. 061

\begin{tabular}{|r|r|}
\hline$r-$ 外 & 100.000 \\
\hline
\end{tabular} 


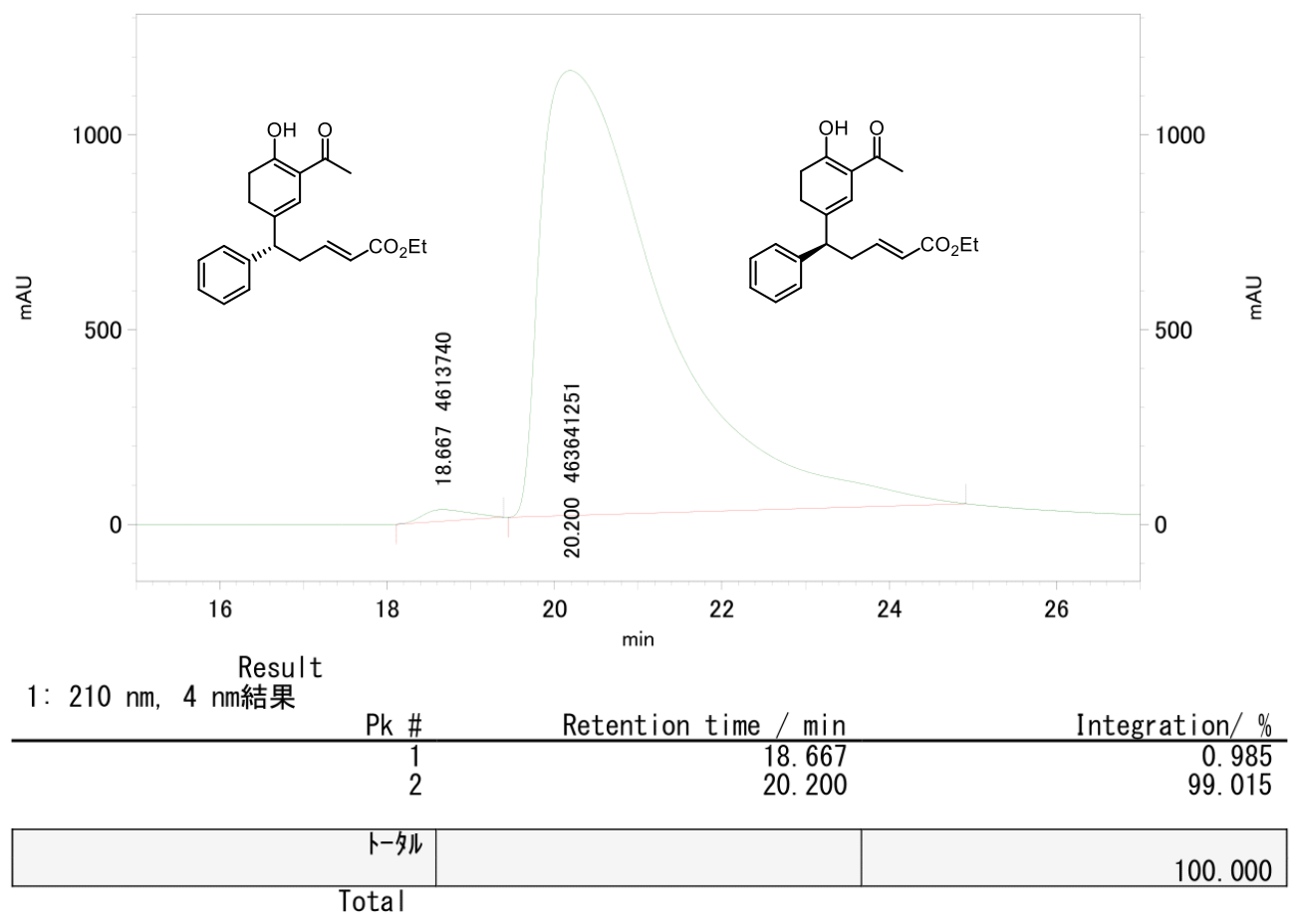

S89 


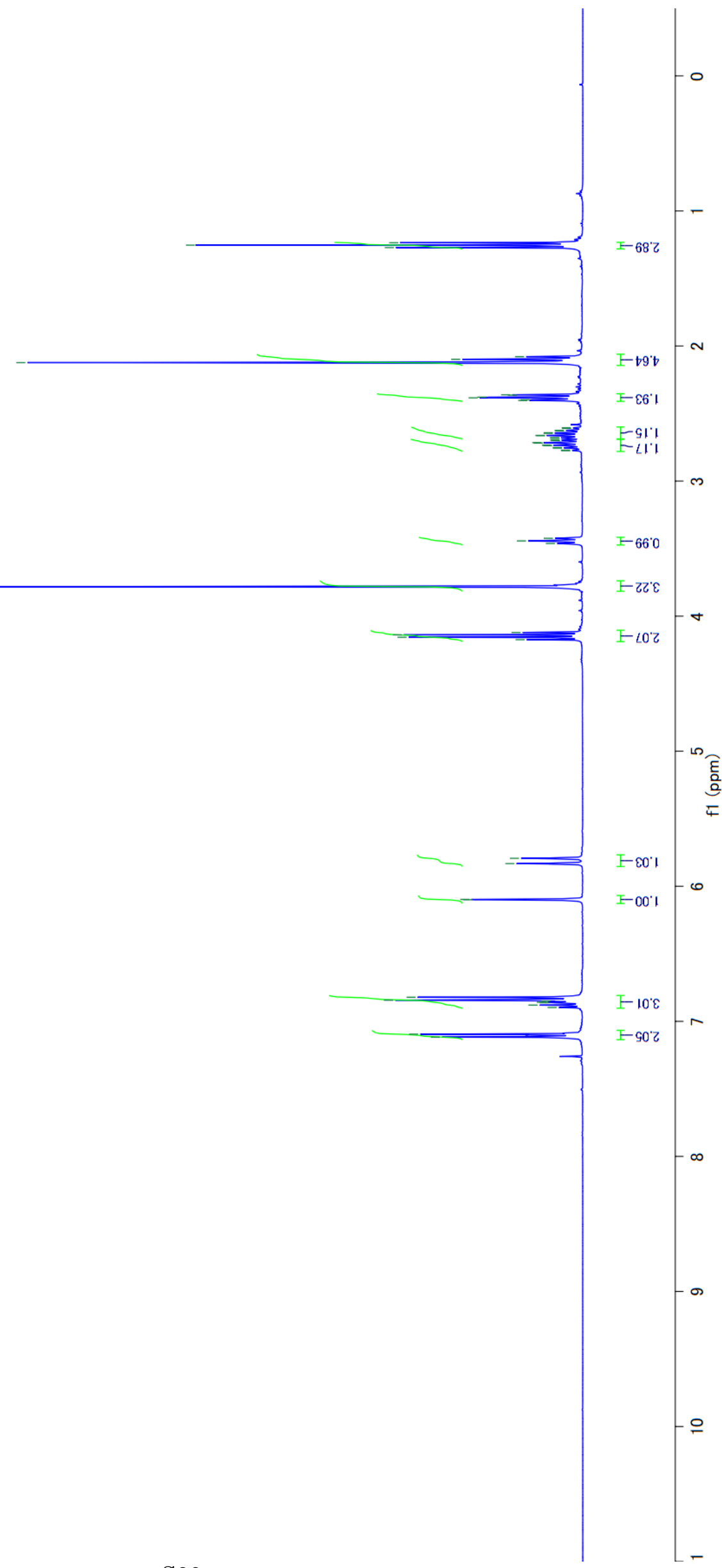



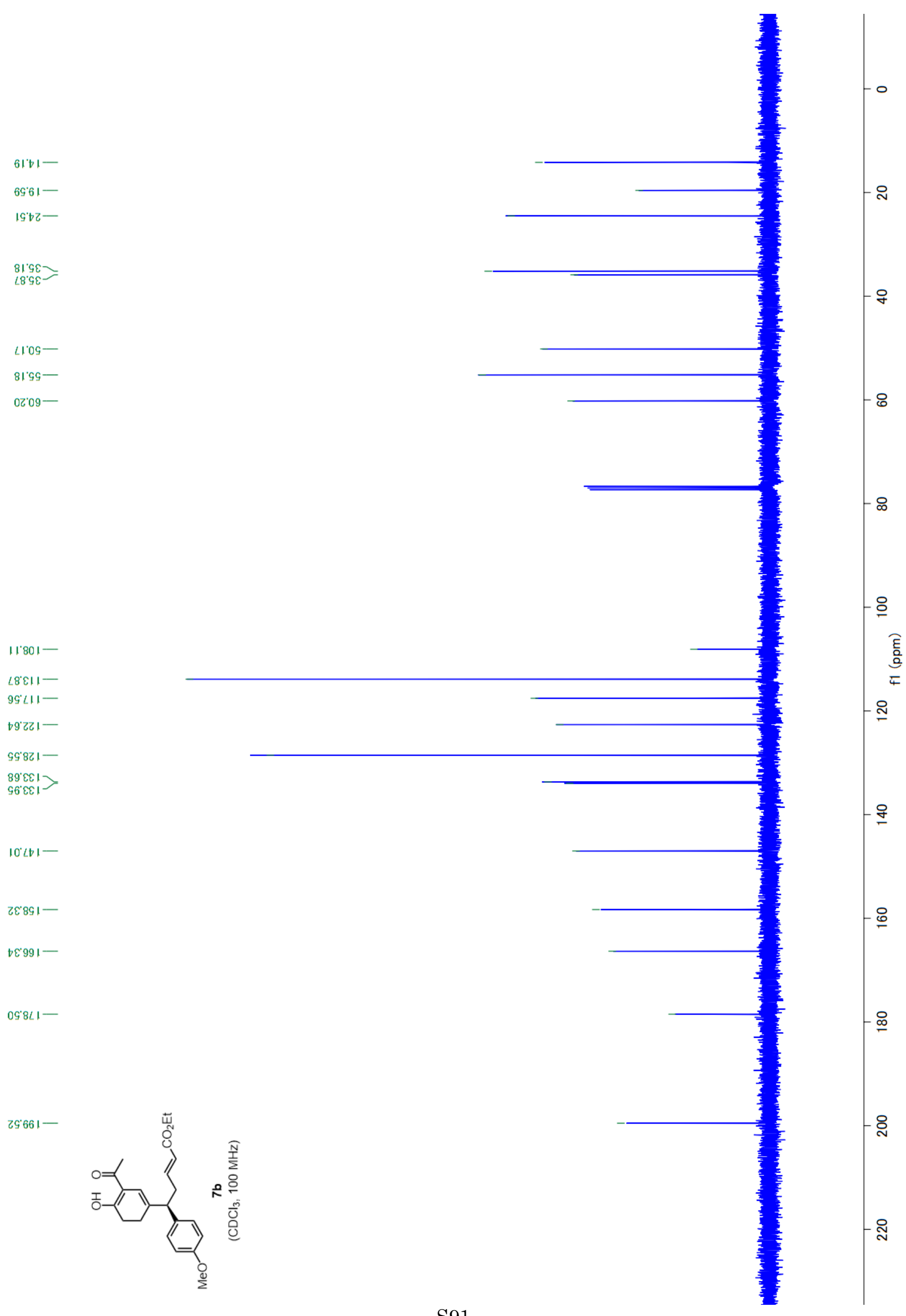


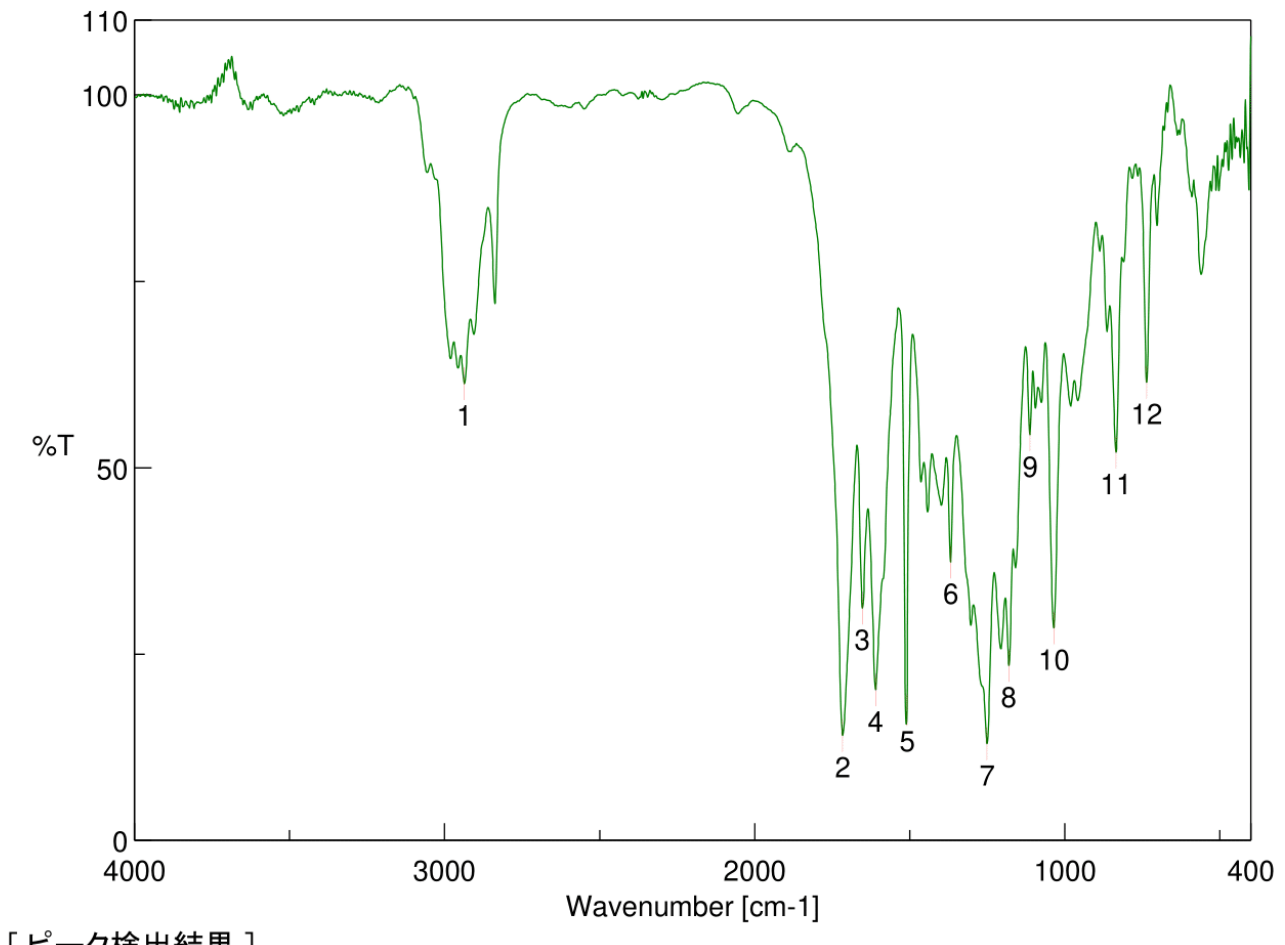

\begin{tabular}{llllll}
\multicolumn{2}{l}{ [ピーク検出結果 ] } & & & \\
No. & 位置 & 強度 & No. & 位置 & 強度 \\
1 & 2936.09 & 61.2598 & 2 & 1716.34 & 14.076 \\
3 & 1652.7 & 31.1147 & 4 & 1610.27 & 20.1704 \\
5 & 1509.03 & 17.5518 & 6 & 1368.25 & 37.3292 \\
7 & 1250.61 & 12.9641 & 8 & 1180.22 & 23.4474 \\
9 & 1112.73 & 54.3861 & 10 & 1035.59 & 28.481 \\
11 & 835.026 & 52.0484 & 12 & 735.71 & 61.4293
\end{tabular}

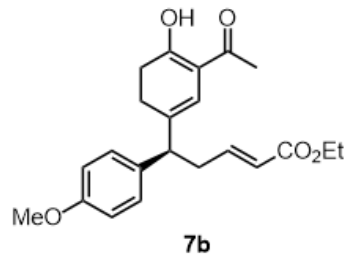




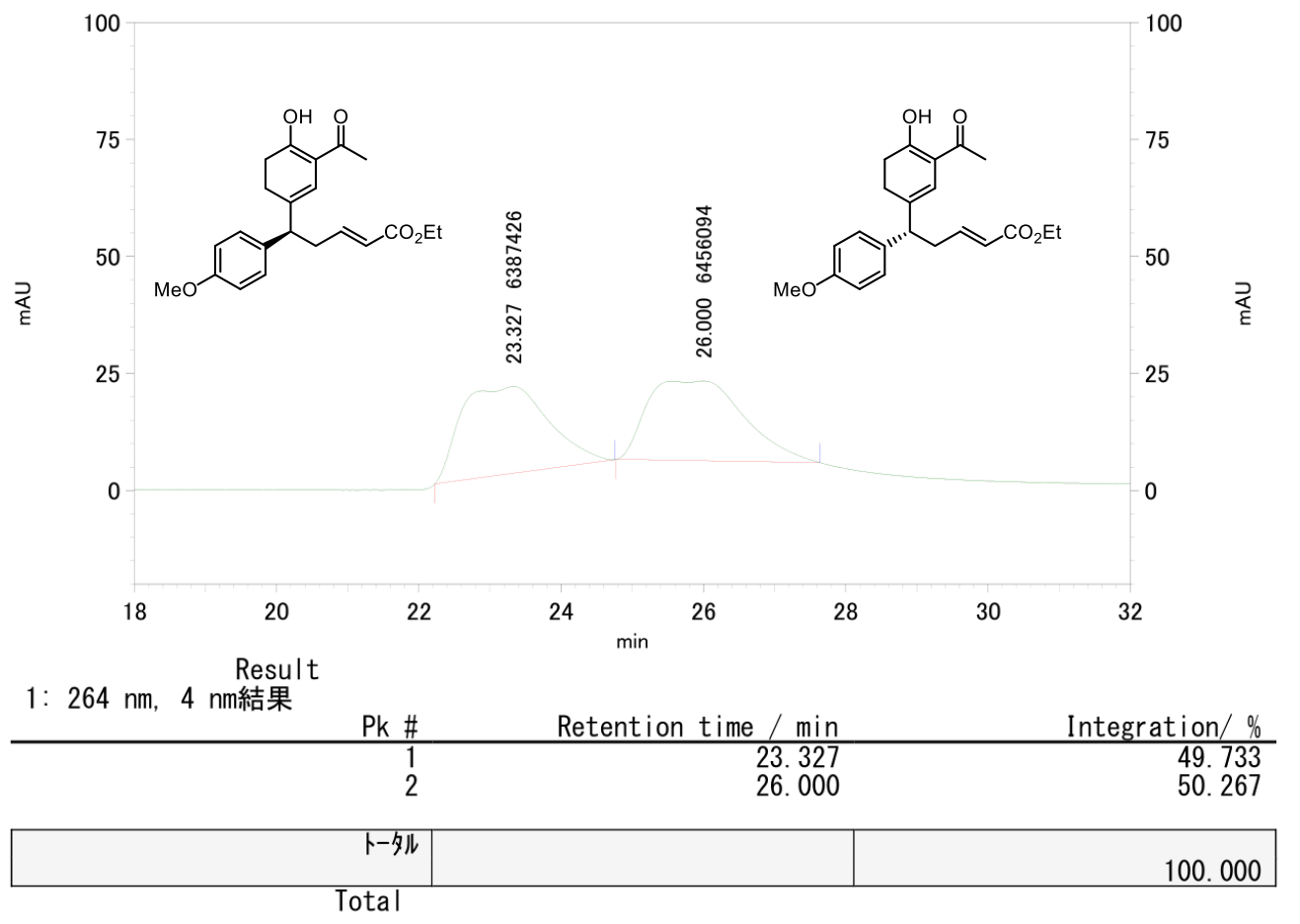




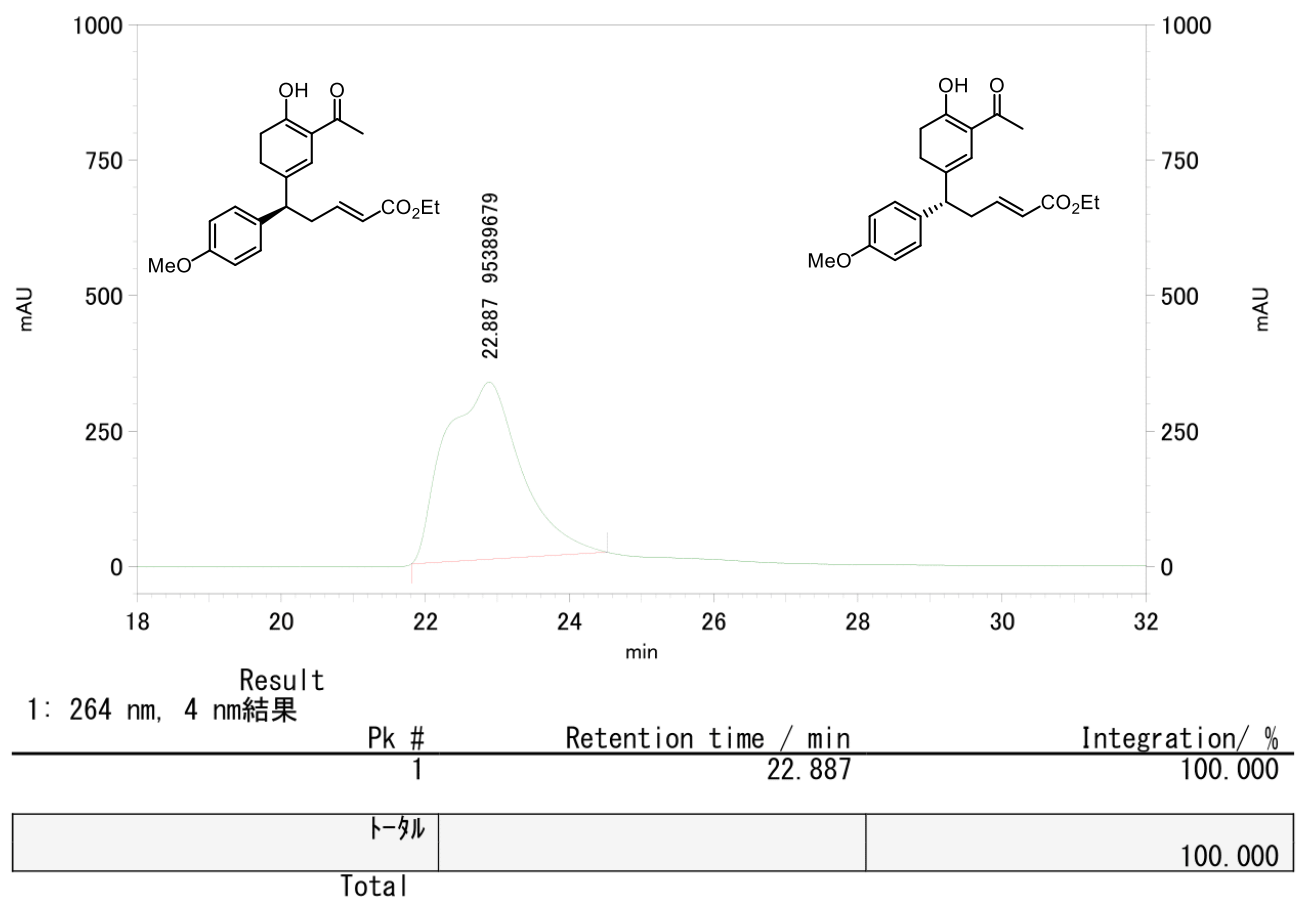




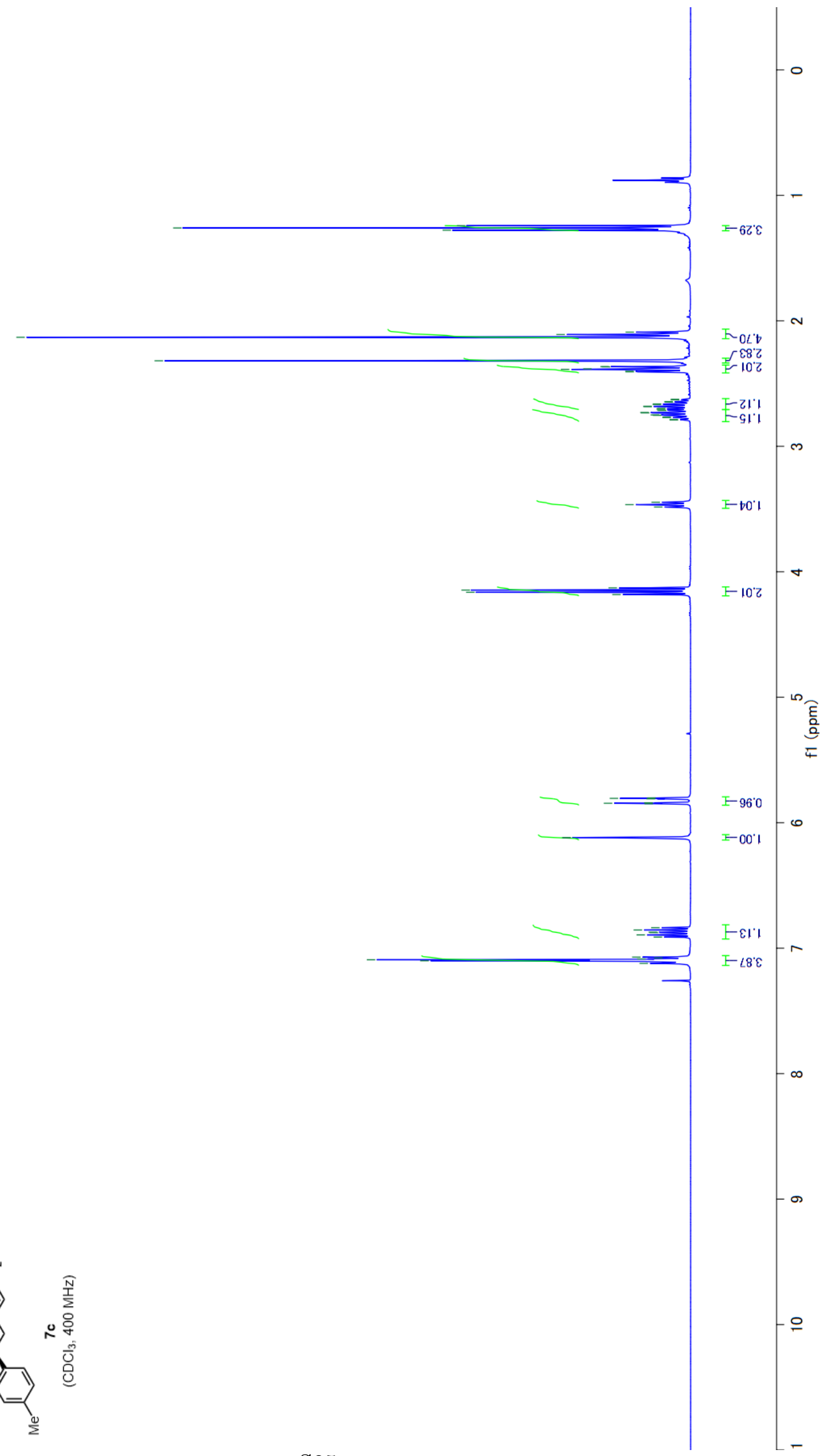




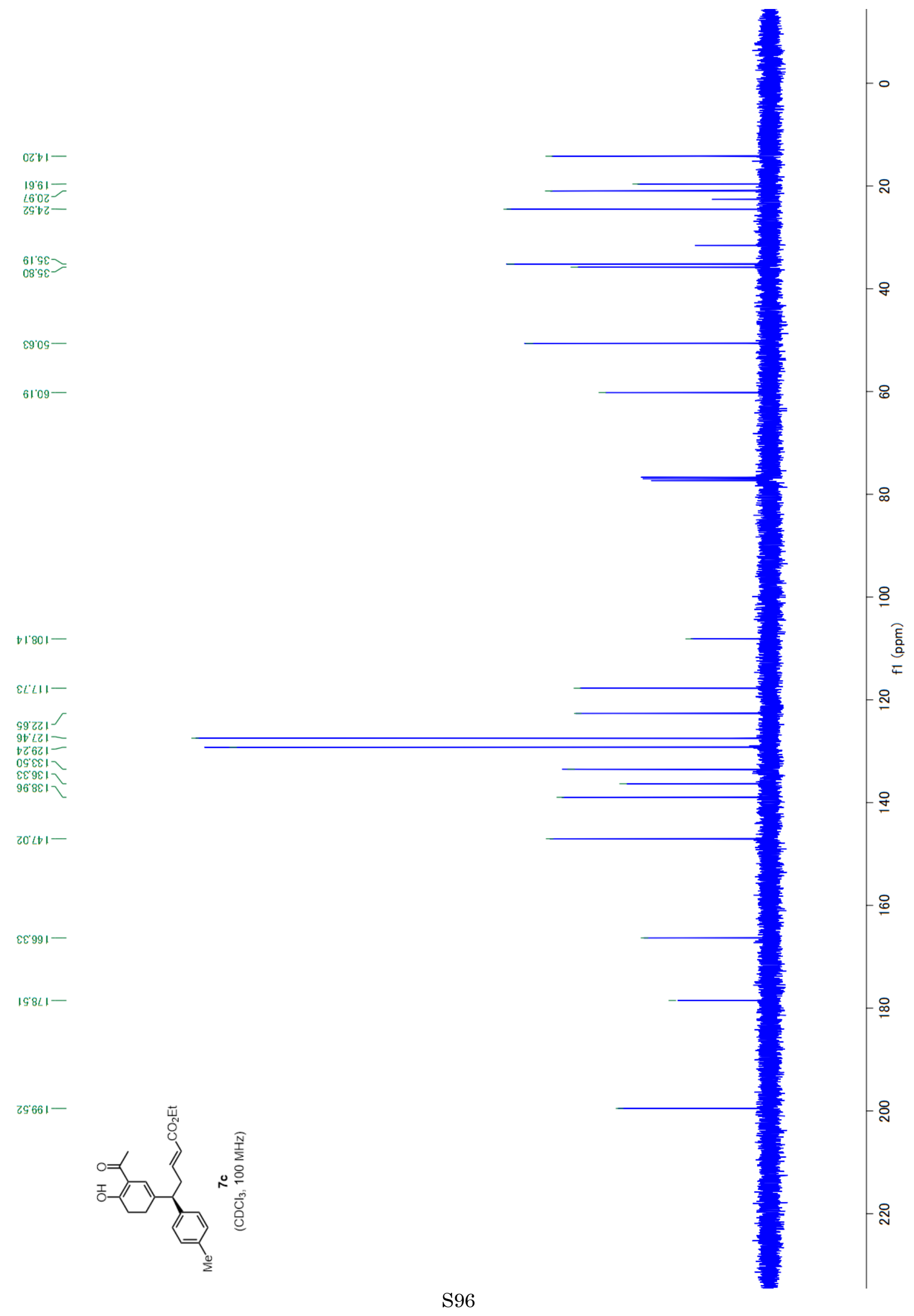




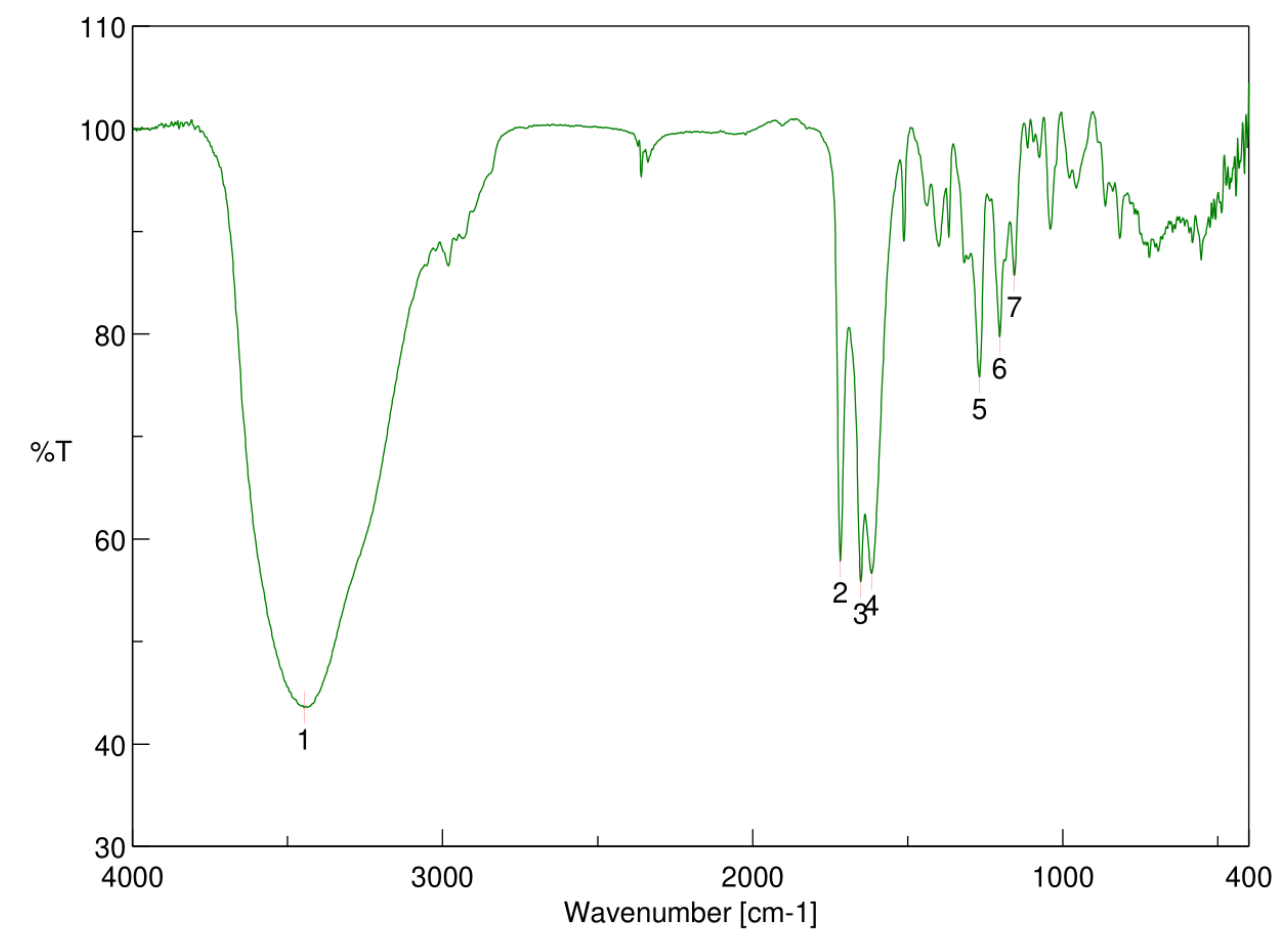

\begin{tabular}{llllll}
\multicolumn{2}{l}{ [ピーク検出結果 ] } & & & & \\
No. & 位置 & 強度 & No. & 位置 & 強度 \\
1 & 3446.17 & 43.5271 & 2 & 1717.3 & 57.8466 \\
3 & 1651.73 & 55.807 & 4 & 1617.02 & 56.5984 \\
5 & 1268.93 & 75.7912 & 6 & 1203.36 & 79.7154 \\
7 & 1156.12 & 85.7069 & & &
\end{tabular}

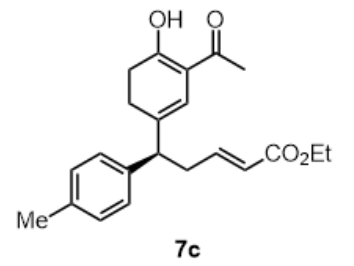




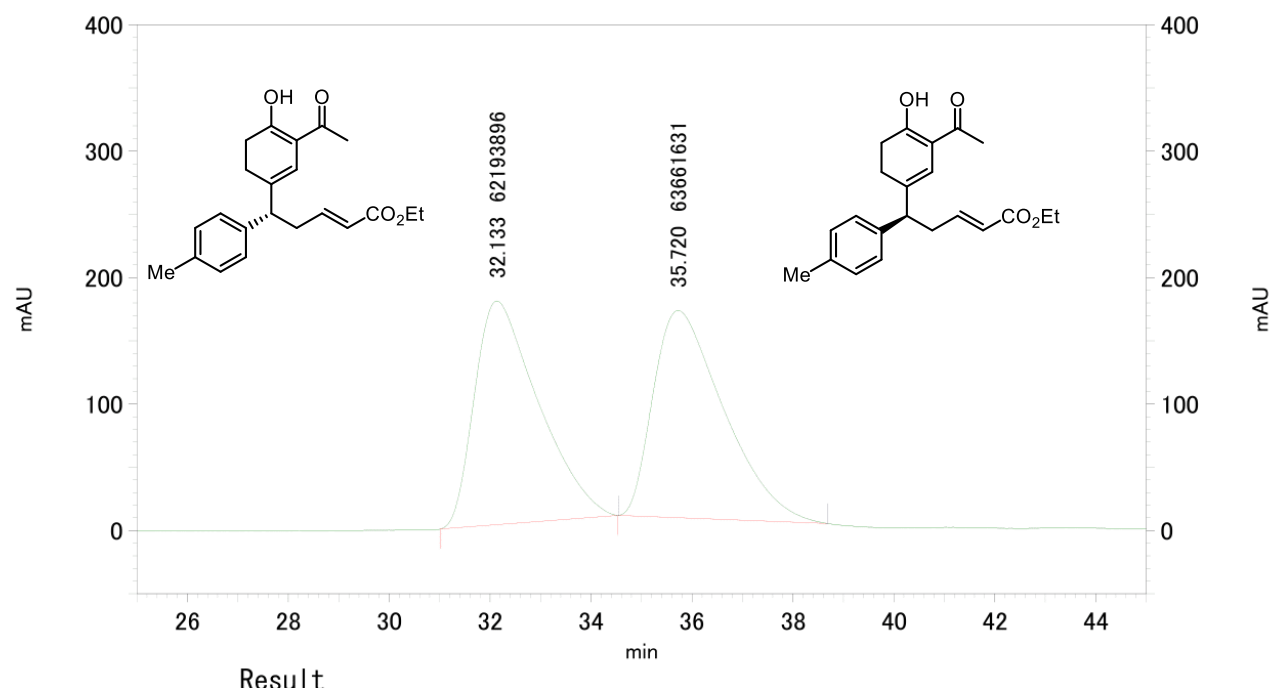

1: $210 \mathrm{~nm}, 4 \mathrm{~nm}$ 結果

Pk \# Retention time / min Integration/ \%

\begin{tabular}{|r|r|r|}
\hline$r-$ 夕ll & 100.000 \\
\hline
\end{tabular}




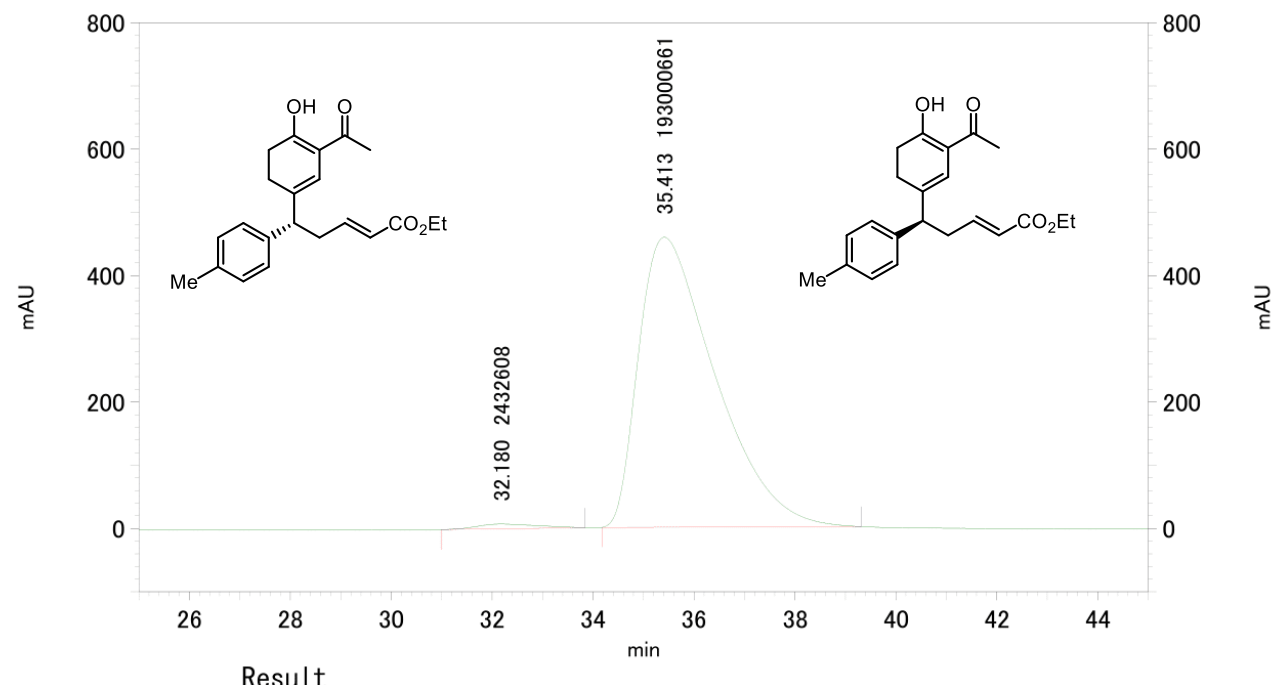

1: $210 \mathrm{~nm}, 4 \mathrm{~nm}$ 結果

Pk \# Retention time / min

Integration/\%

32. 180

98. 755

Total

100. 000 


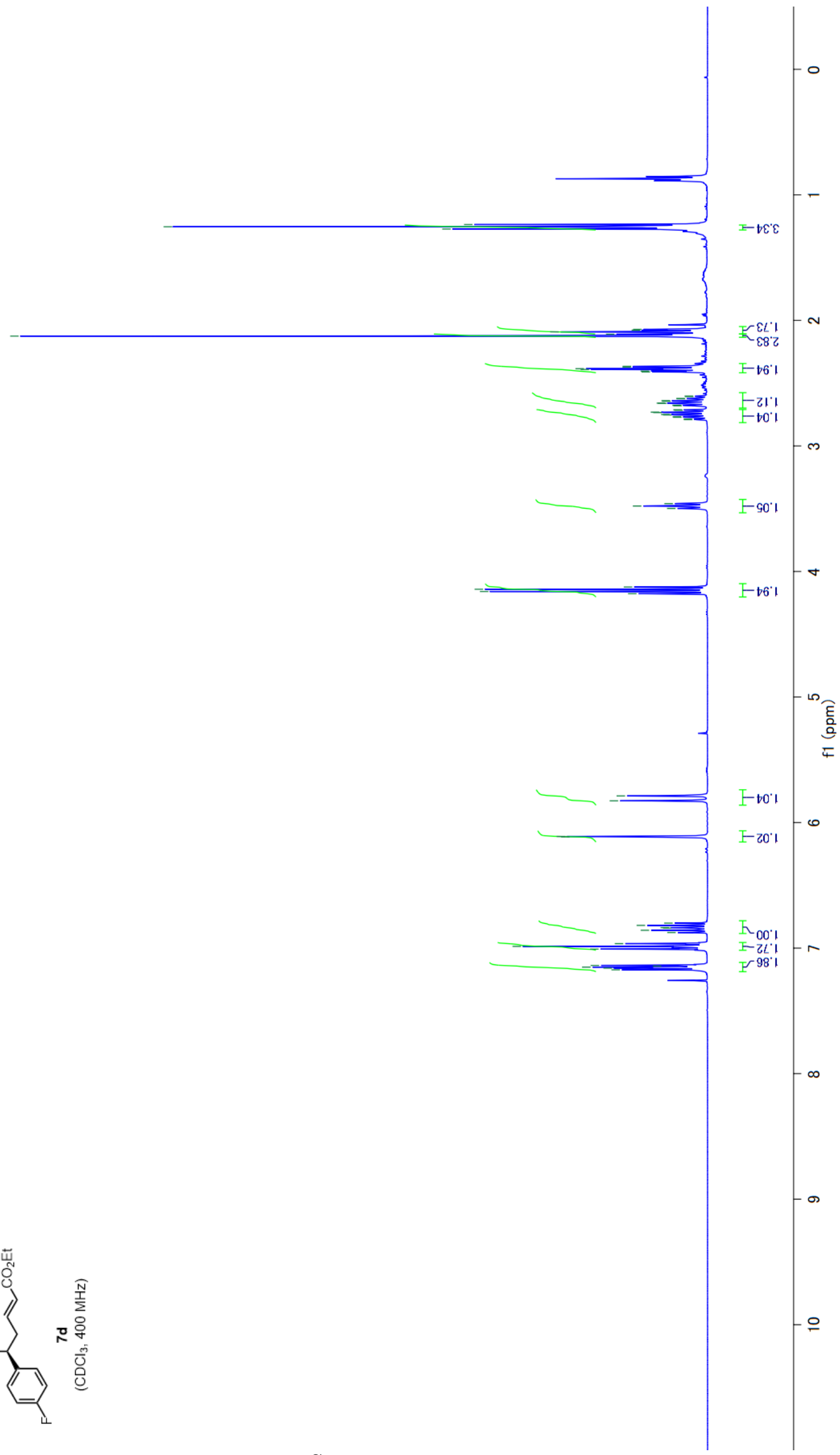




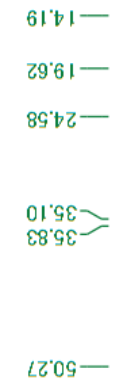

LZ09-

$20801-$

$87911>$
$6 t^{\circ} 911$

ع0.811-

เ62

$80621-$

G० हू।

G9 $L E I]$

st $901-$

65091
$88.291-$

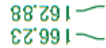

Z8. $8 L 1-$

$67661-$

(u)

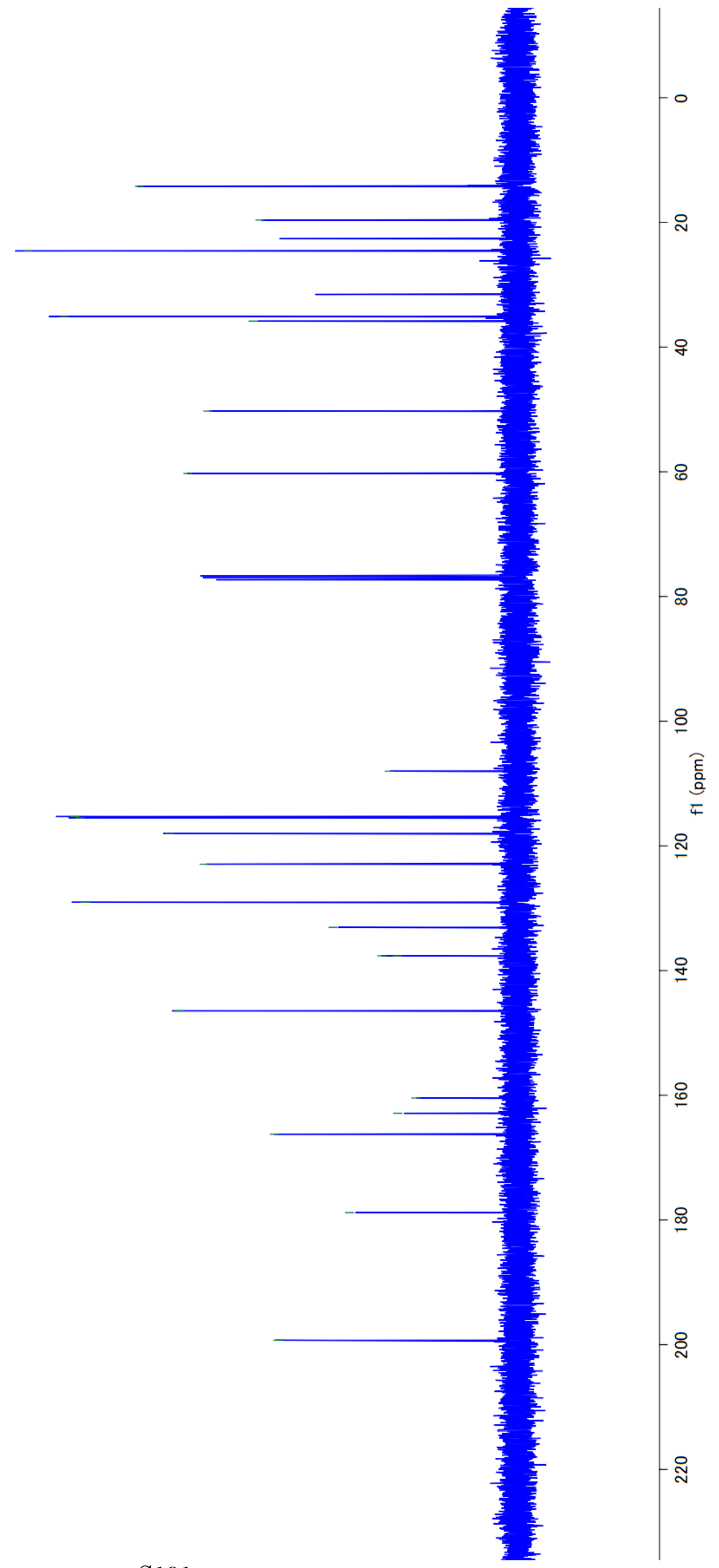

S101 


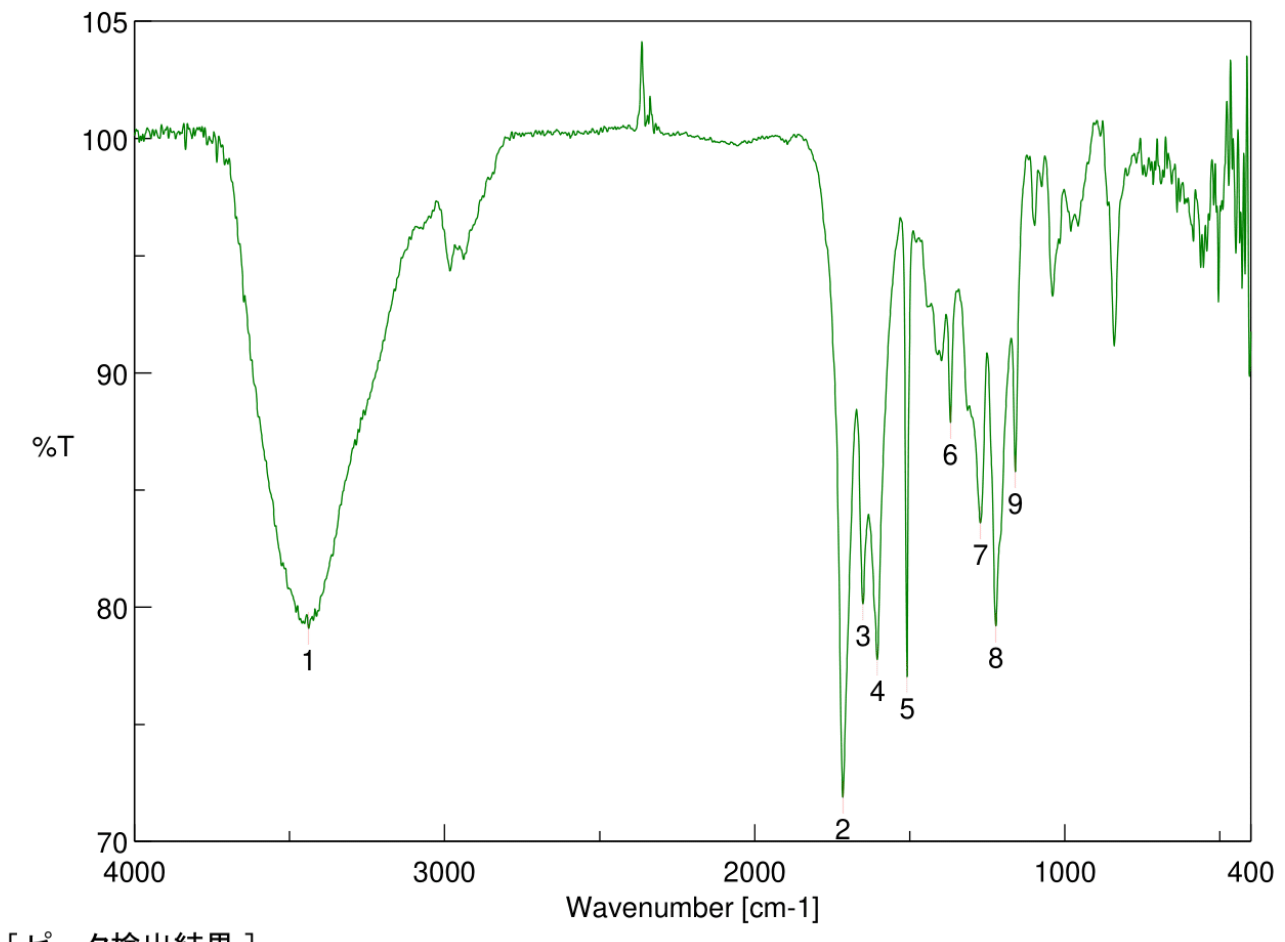

\begin{tabular}{llllll}
\multicolumn{2}{l}{ [ピーク検出結果 ] } & 強度 & No. & 位置 & 強度 \\
No. & 位置 & 39.0952 & 2 & 1715.37 & 71.8747 \\
1 & 3438.46 & 79.074 & 4 & 1604.48 & 77.7607 \\
3 & 1650.77 & 80.1343 & 6 & 1369.21 & 87.8777 \\
5 & 1509.03 & 77.016 & 8 & 1222.65 & 79.1858 \\
7 & 1271.82 & 83.5858 & & &
\end{tabular}

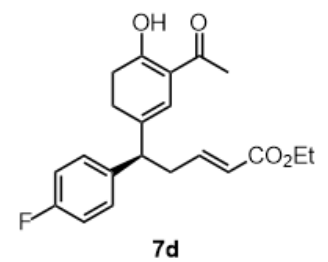




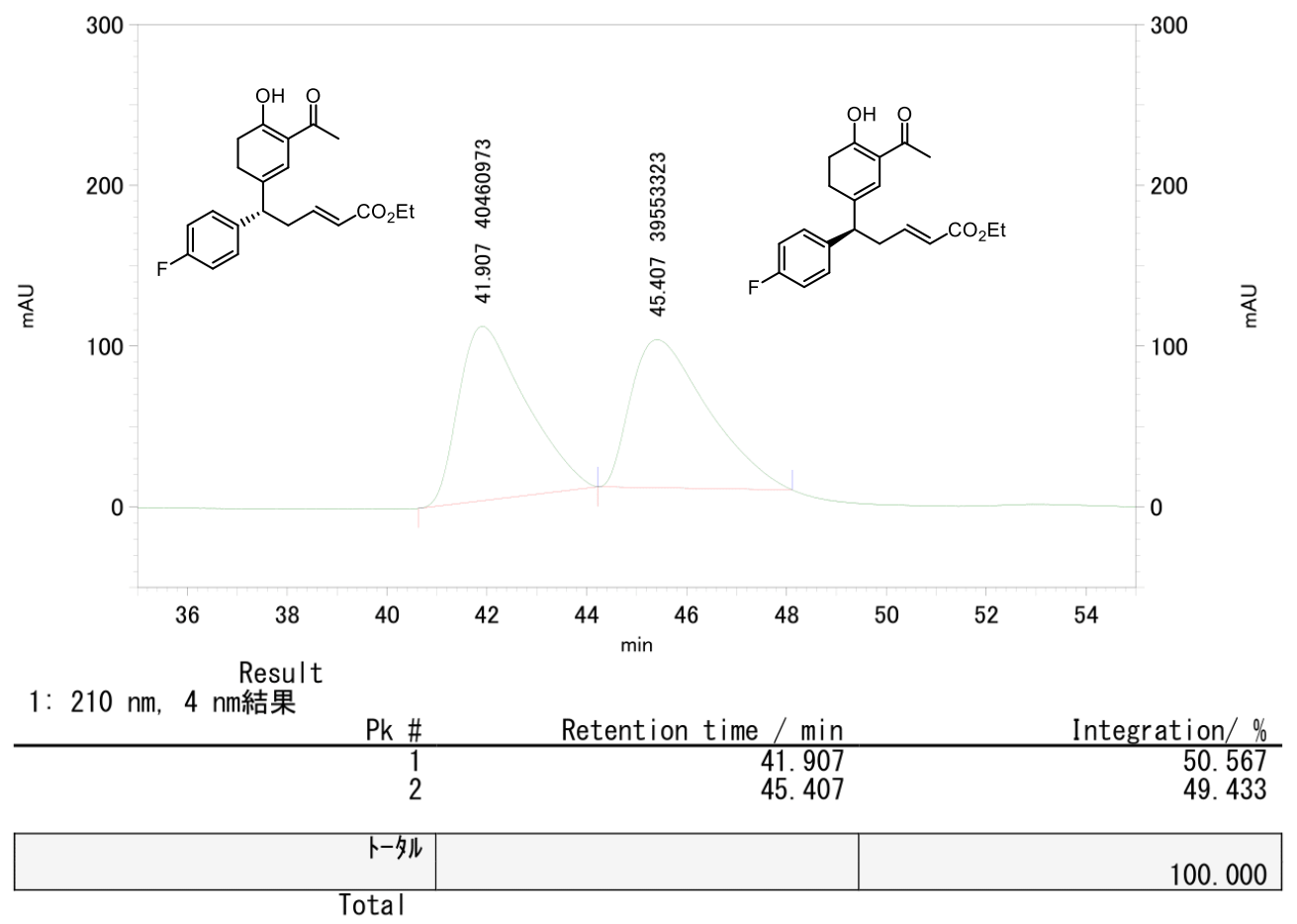




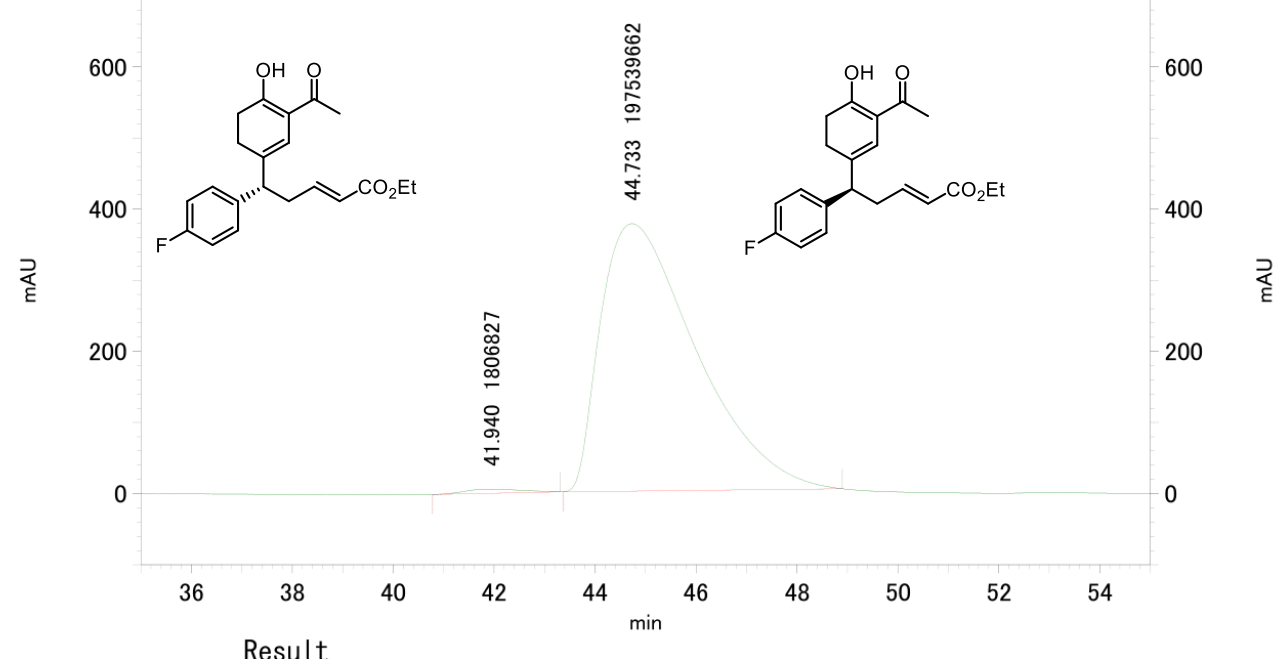

1: $210 \mathrm{~nm}, 4 \mathrm{~nm}$ 結果

Pk \# Retention time / min

Integration/\%

41.940

99. 094

\begin{tabular}{|r|r|r|}
\hline$r-$ 夕ll & 100.000 \\
\hline
\end{tabular} 
sel't

Esl't

B.t.

$96 L^{\circ} \mathrm{G}$
$\mathrm{G}: \mathrm{S}$

हЪ।'9-

$\left.66 \iota^{\circ} 9\right]$

$\left.\angle 18^{\circ} 9\right]$

ตระ.

$\rightarrow \angle 8^{\circ} 9$

921.2

697' $L$ 厂

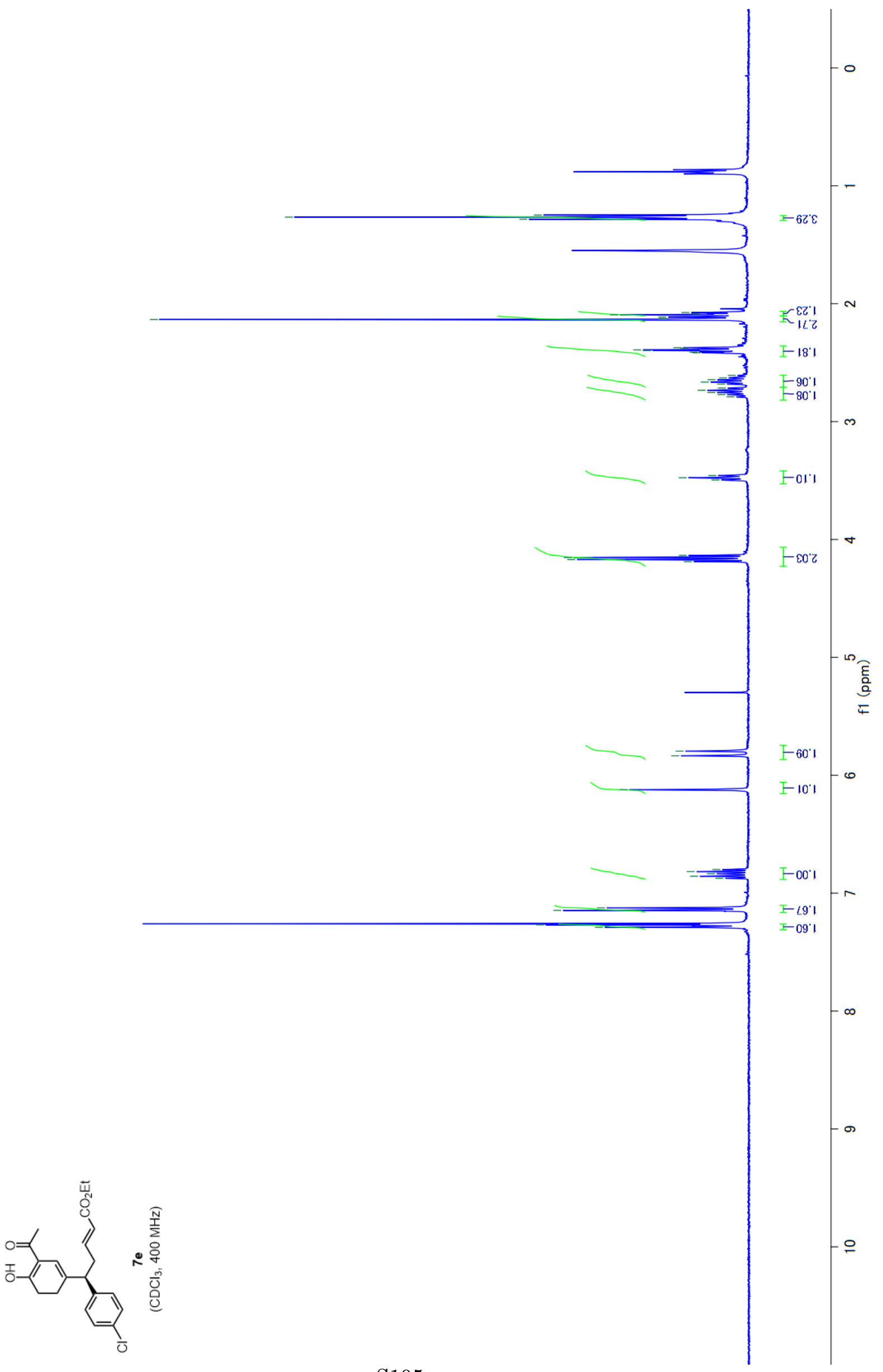


80.61
81.61

๕961-

tctoz-

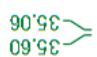

$6 \varepsilon^{\circ} 09$

L209-

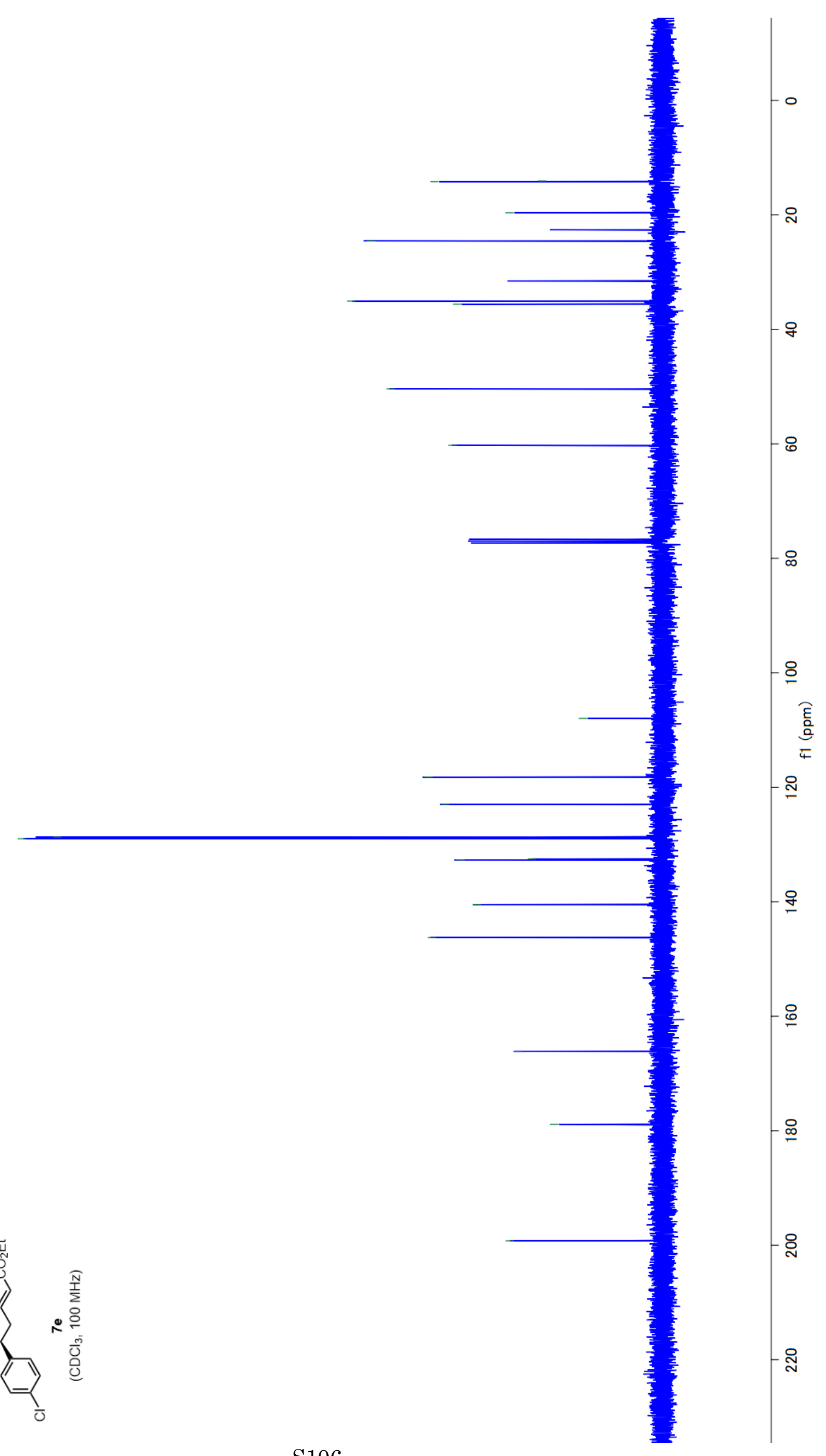

๕乙66เ-

(-) 


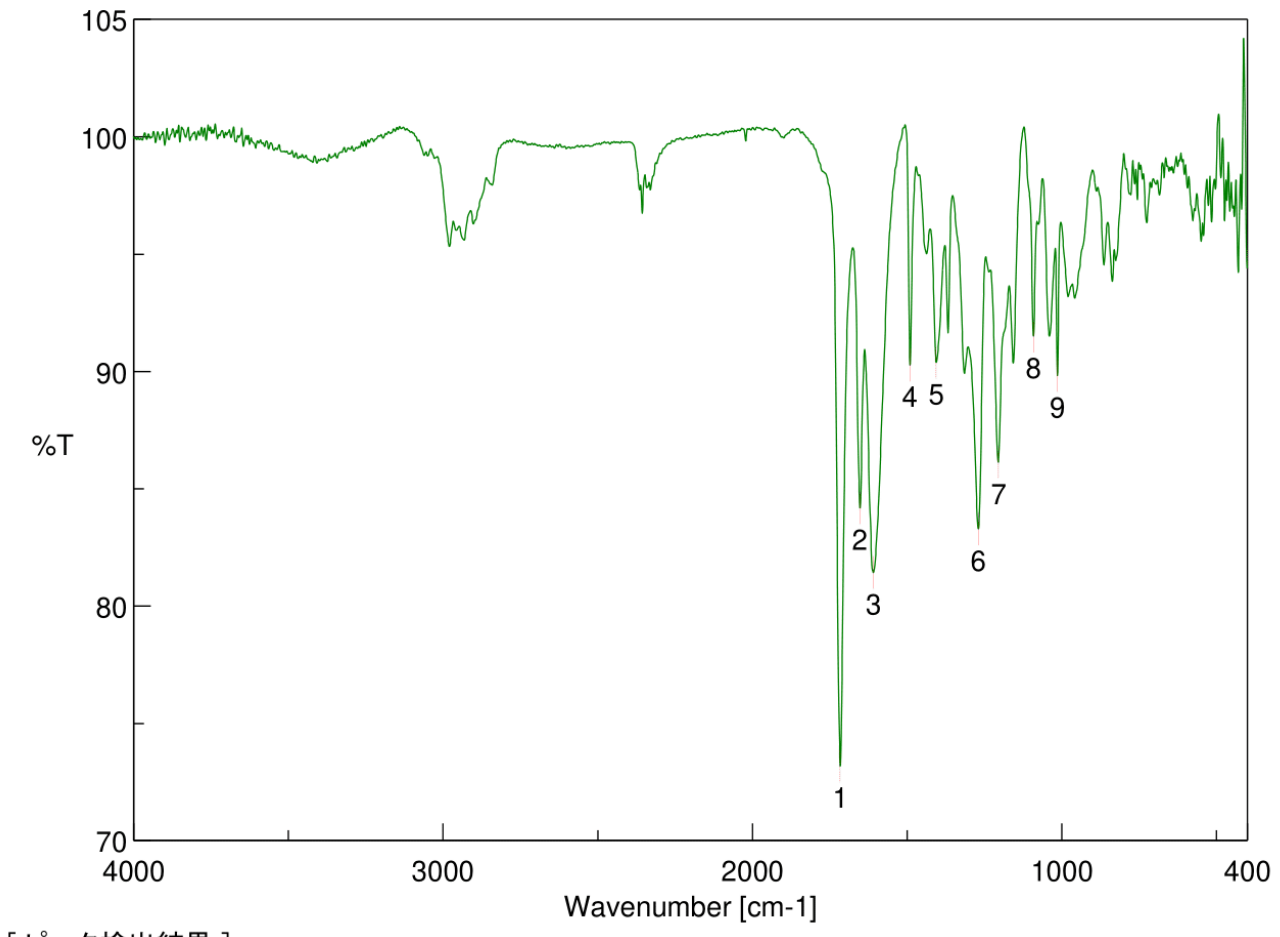

\begin{tabular}{llllll}
\multicolumn{2}{l}{$\left[\begin{array}{l}l \\
\text { [ピーク検出結果 ] }\end{array}\right.$} & No. & & \\
No. & 位置 & 強度 & 位置 & 強度 \\
1 & 1716.34 & 73.1773 & 2 & 1652.7 & 84.1788 \\
3 & 1609.31 & 81.4228 & 4 & 1490.7 & 90.2545 \\
5 & 1405.85 & 90.3721 & 6 & 1269.9 & 83.2827 \\
7 & 1205.29 & 86.1251 & 8 & 1091.51 & 91.4953 \\
9 & 1014.37 & 89.807 & & &
\end{tabular}

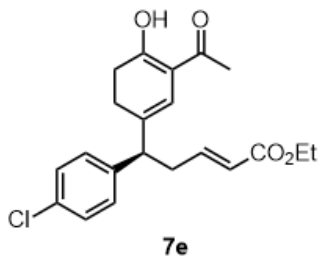




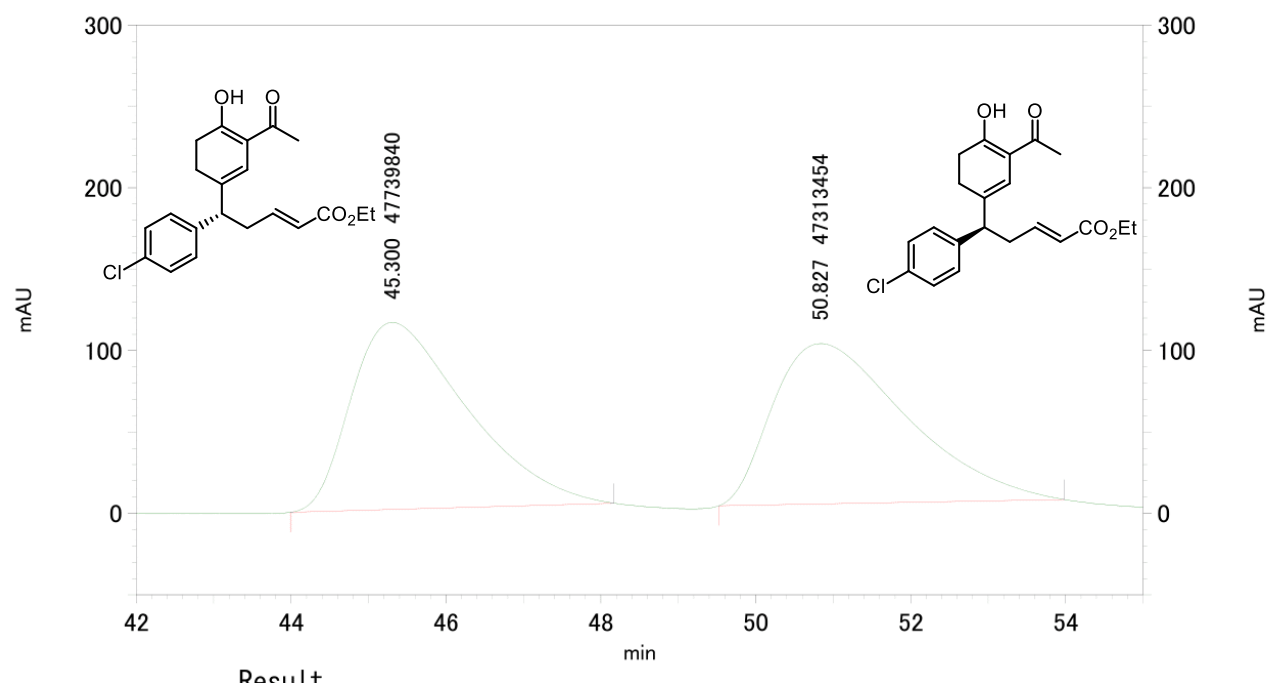

1: $210 \mathrm{~nm}, 4$ nm結果

Pk \# Retention time / min

Integration/\%

45.300

50.224
49.776

\begin{tabular}{|r|r|r|}
\hline$r-$ 外 & 100.000 \\
\hline
\end{tabular} 


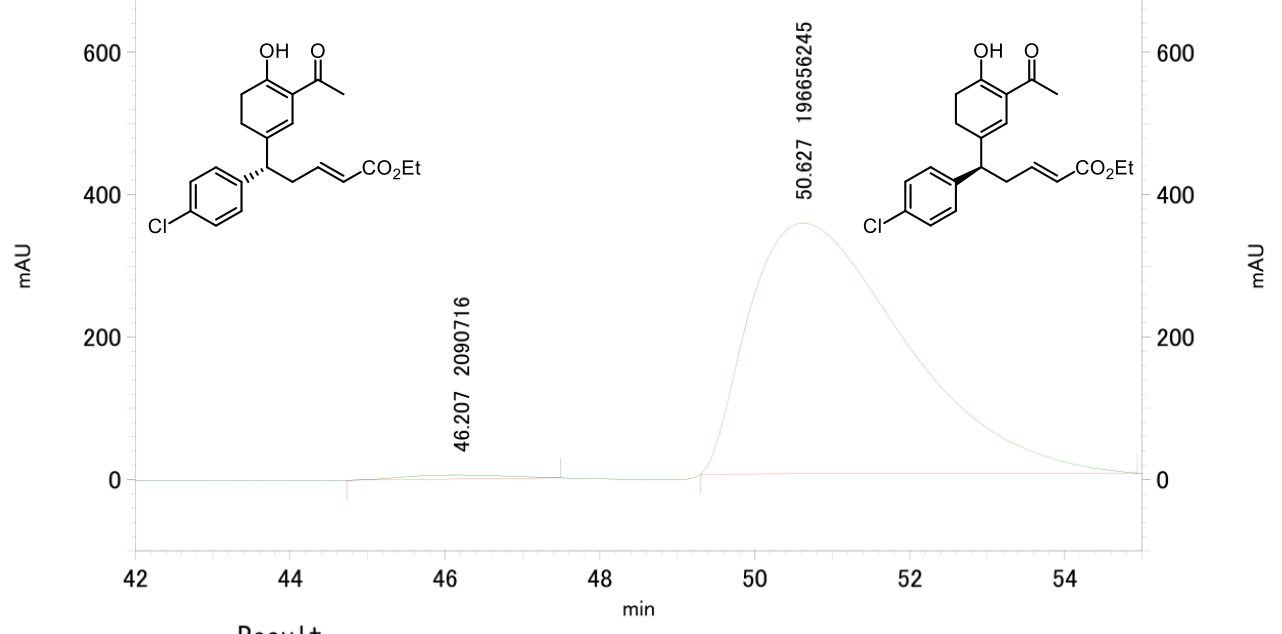

1: $210 \mathrm{~nm}, 4 \mathrm{~nm}$ 結果

Pk \# Retention time / min

Integration/\%

46.207

98.948

\begin{tabular}{|r|r|r|}
\hline$r-$ 外 & 100.000 \\
\hline
\end{tabular} 

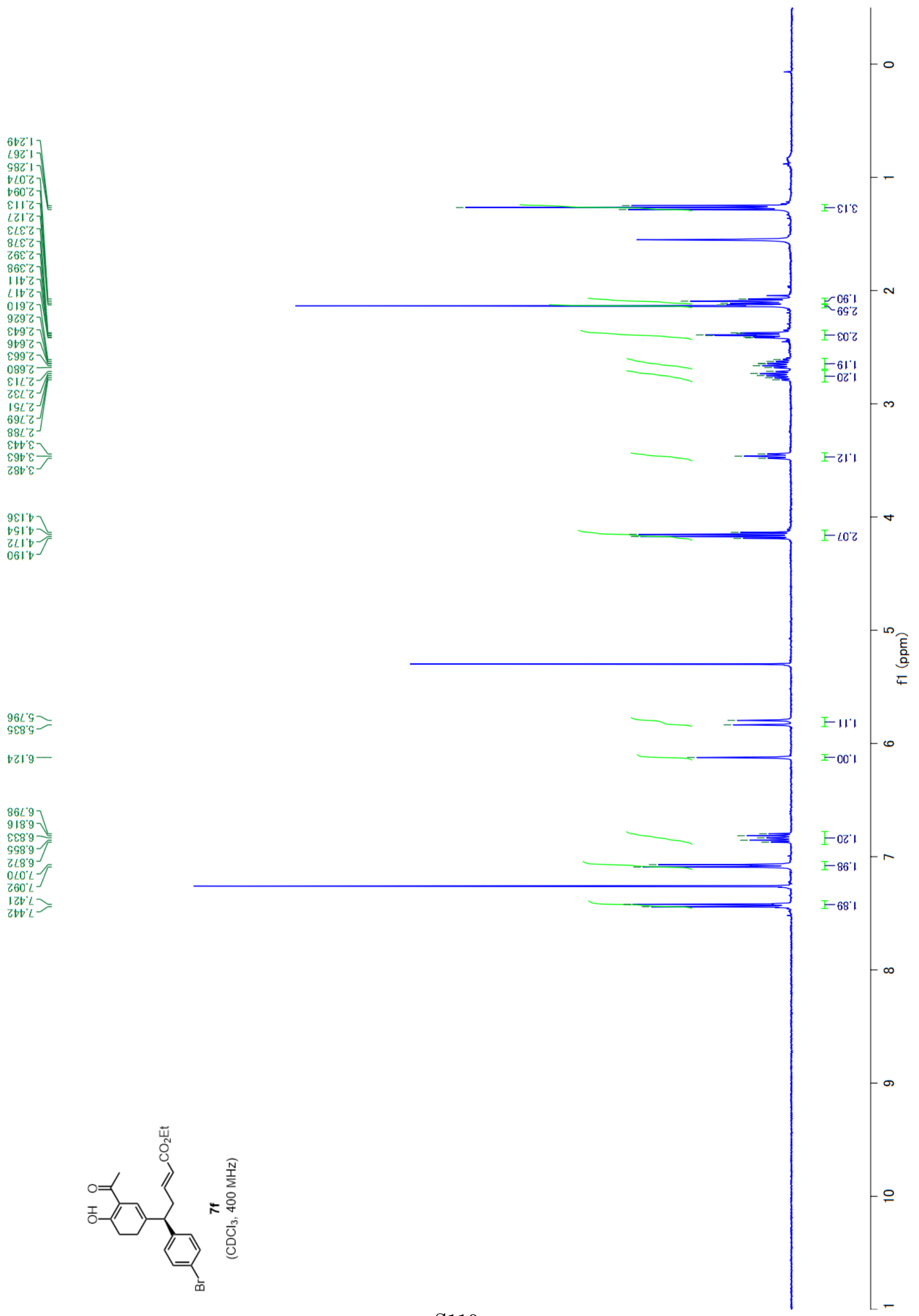

$96 L \mathrm{G}$
$\mathrm{G}$

เל1. 9 -

$86 L^{\circ} 9$

$918^{\circ}-$

हE8. $9-$

$98^{\circ} 9^{\circ}$
乙

$0<0 \%$

$260^{\circ} \mathrm{L}$

120.
$2 t 2$ 


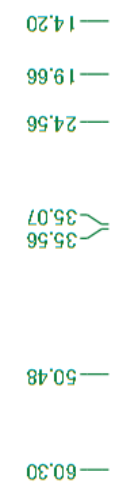

0ะ.09-

$66.201-$

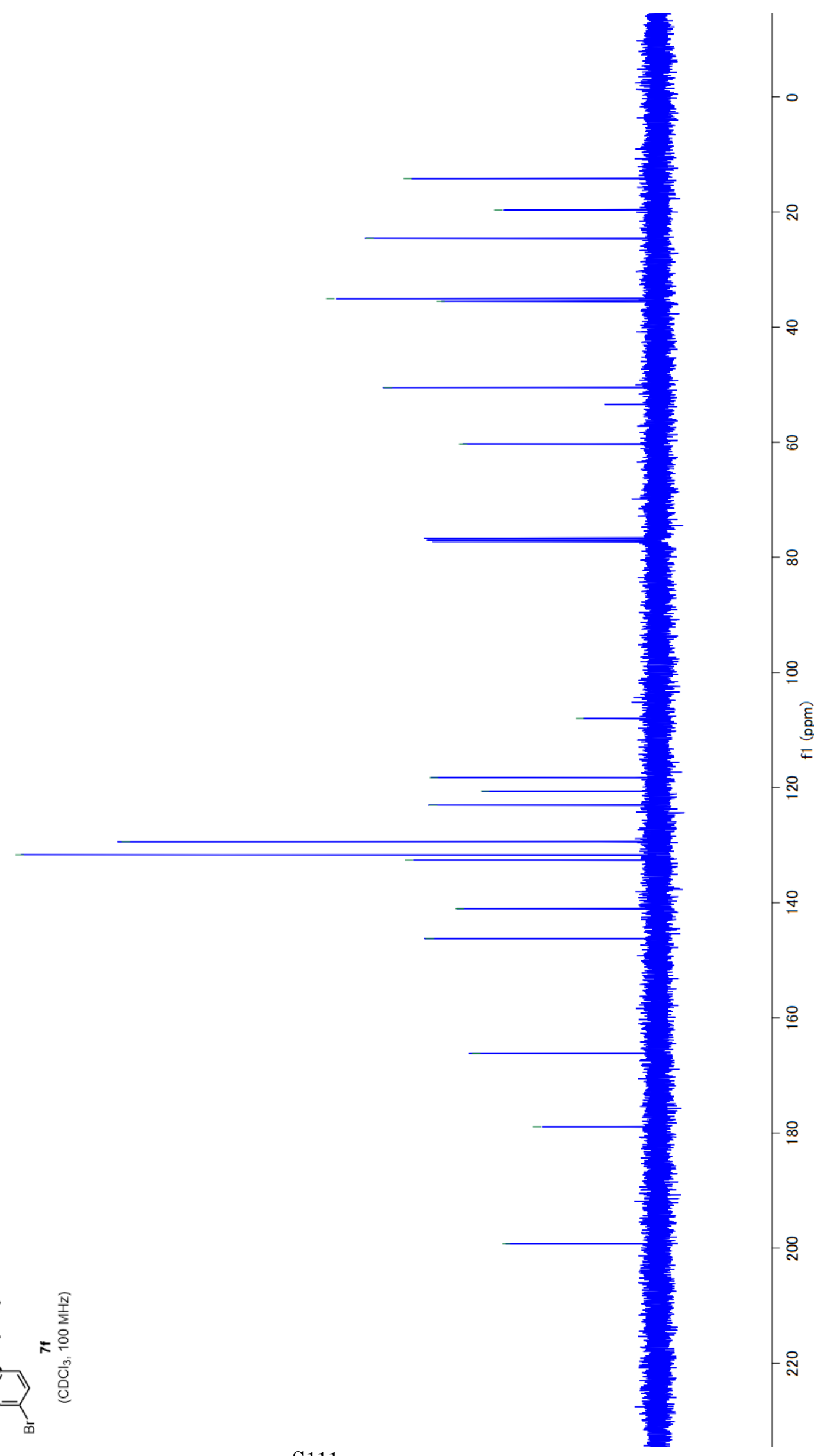

$67811-$

ร9.0Z1-

$68621-$

$89^{\circ}|\varepsilon|=$
$\left|9^{\circ} \mathrm{ZE}\right|=$

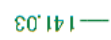

Zว 9 เ 1 -

E6 $8 L 1-$

๕ช $661-$

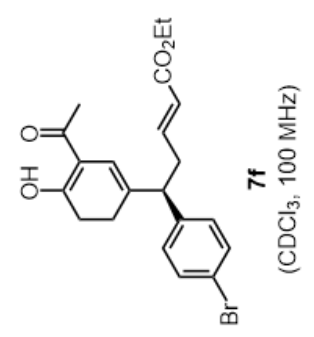




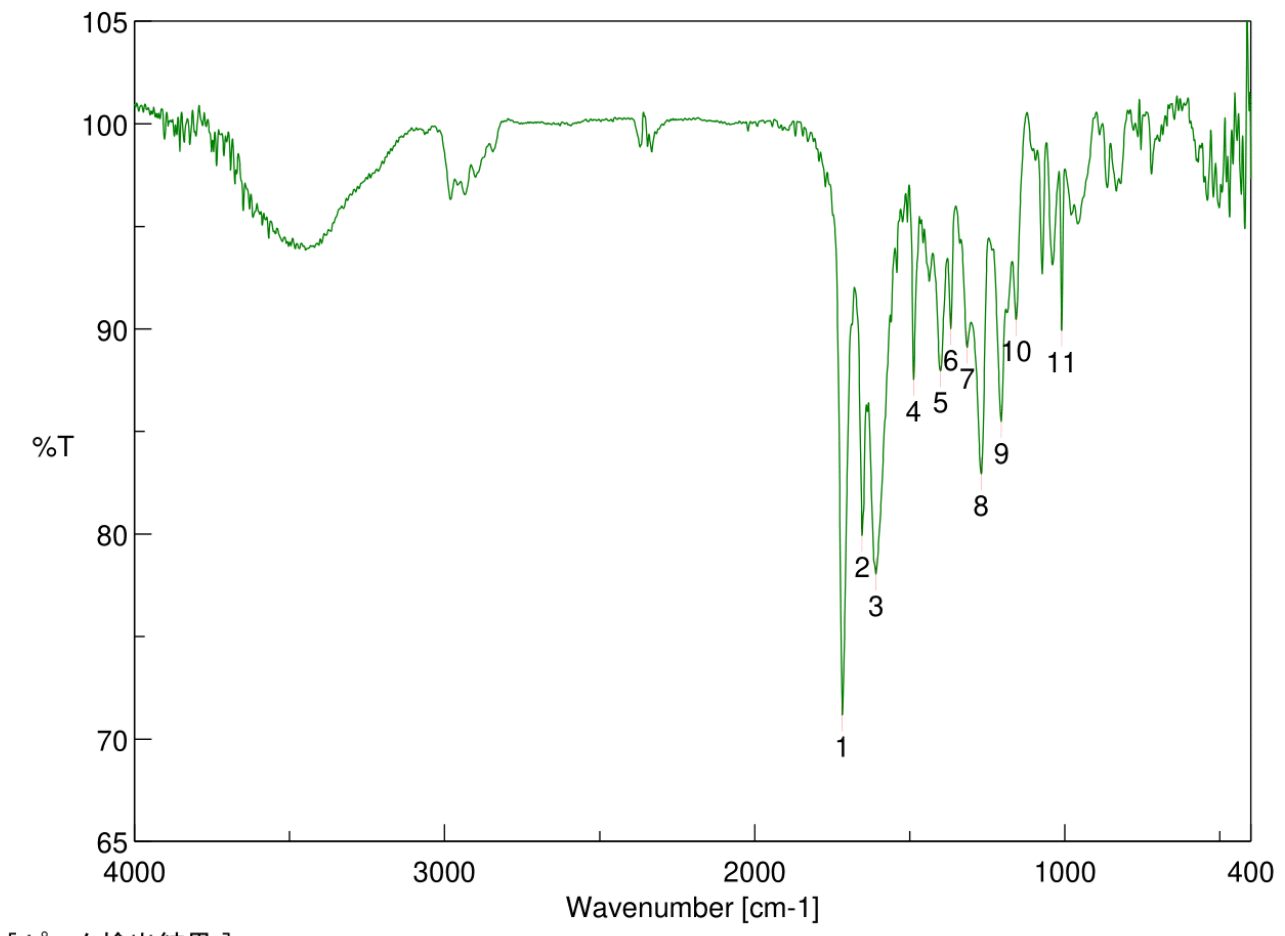

\begin{tabular}{llllll}
\multicolumn{2}{l}{ [ピーク検出結果 ] } & & & & \\
No. & 位置 & 強度 & No. & 位置 & 強度 \\
1 & 1717.3 & 71.1647 & 2 & 1653.66 & 79.9127 \\
3 & 1609.31 & 78.0402 & 4 & 1487.81 & 87.5213 \\
5 & 1401.03 & 87.9562 & 6 & 1367.28 & 90.0057 \\
7 & 1315.21 & 89.1014 & 8 & 1269.9 & 82.9273 \\
9 & 1205.29 & 85.4775 & 10 & 1157.08 & 90.4566 \\
11 & 1009.55 & 89.9166 & & &
\end{tabular}

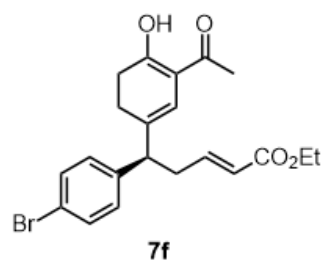




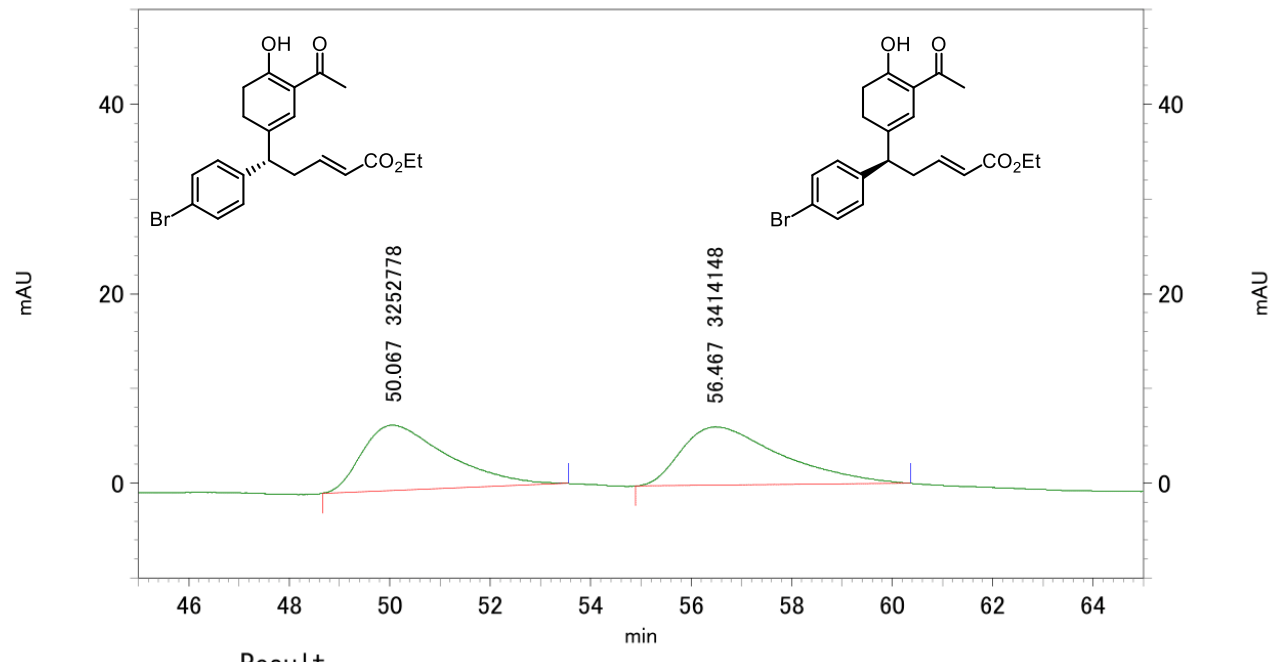

1: $319 \mathrm{~nm}, 4 \mathrm{~nm}$ 結果

Pk \# Retention time / min

50.067

Integration/\%

56. 467

51. 210

\begin{tabular}{|r|r|}
\hline$r-$ 外 & 100.000 \\
\hline
\end{tabular} 


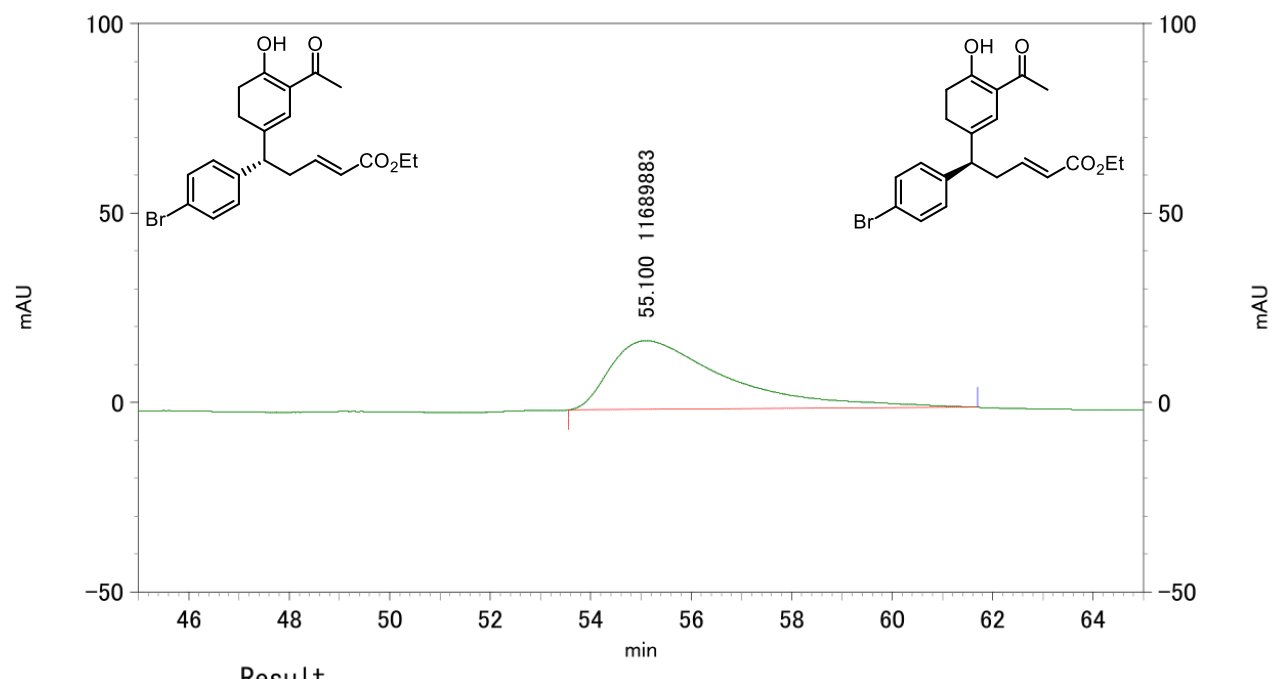

1: $319 \mathrm{~nm}, 4$ nm結果

Pk \# Retention time / min Integration/\%

\begin{tabular}{|r|r|}
\hline$r-$ 多 & 100.000 \\
\hline
\end{tabular}




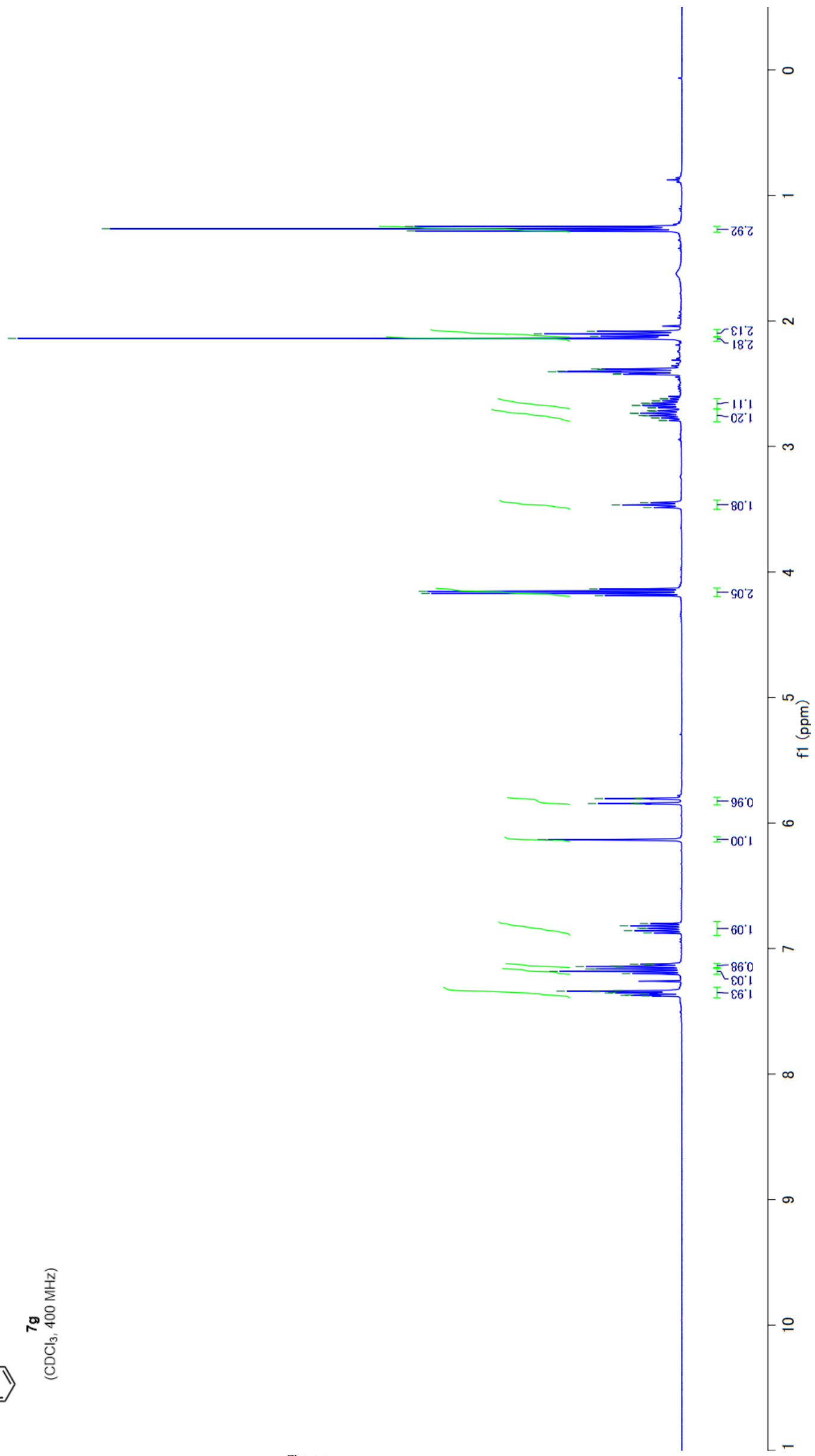




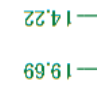

$1902-$

$90,9 \varepsilon$
$7 \varsigma^{\circ} 9$

SLOS-

๕ะ०09-

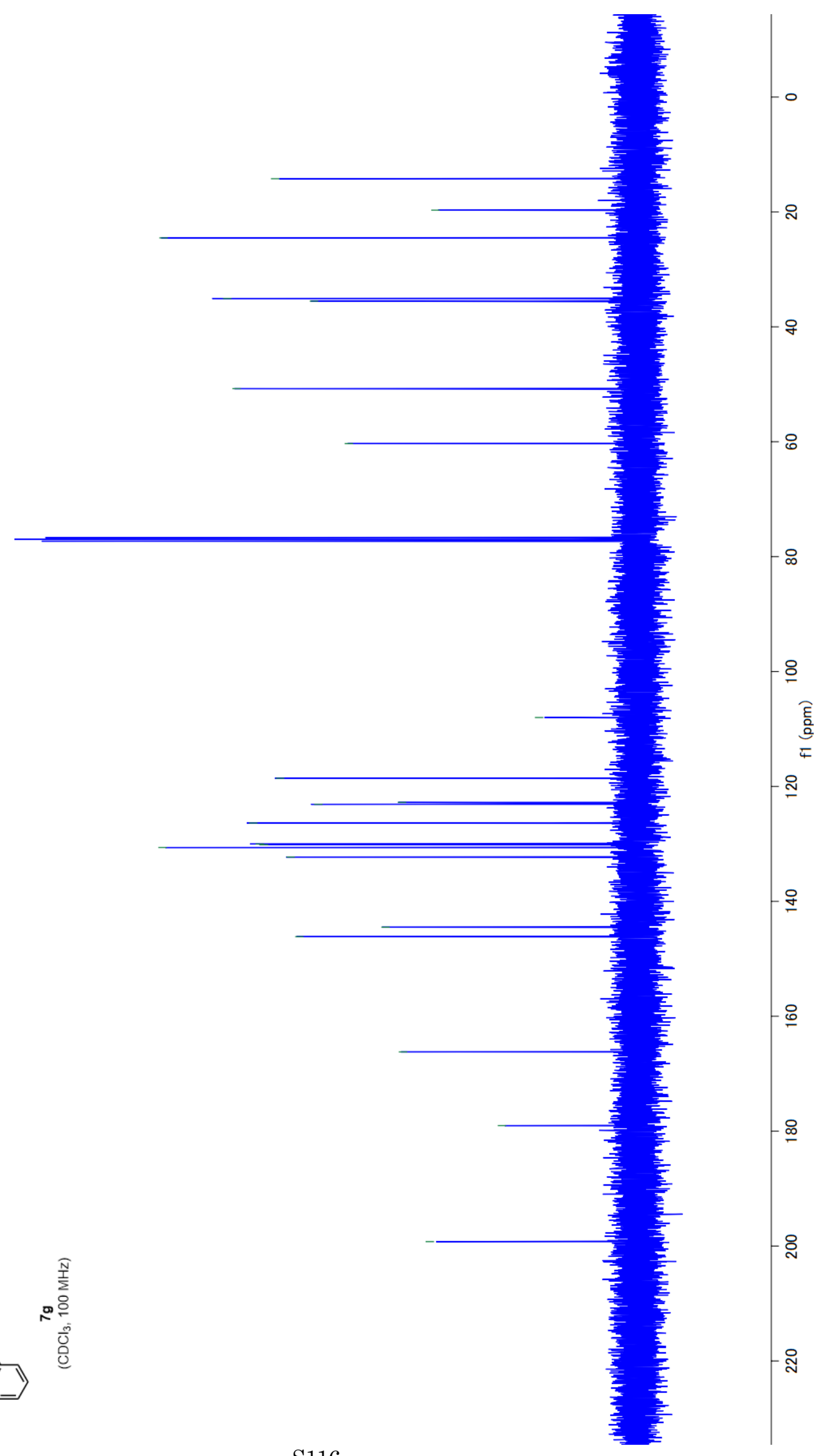

๕ช $661-$

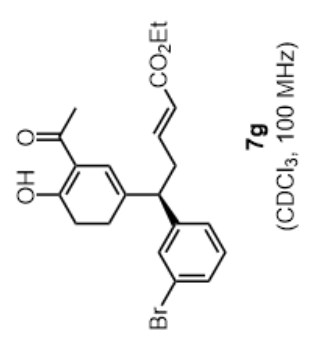

S116 


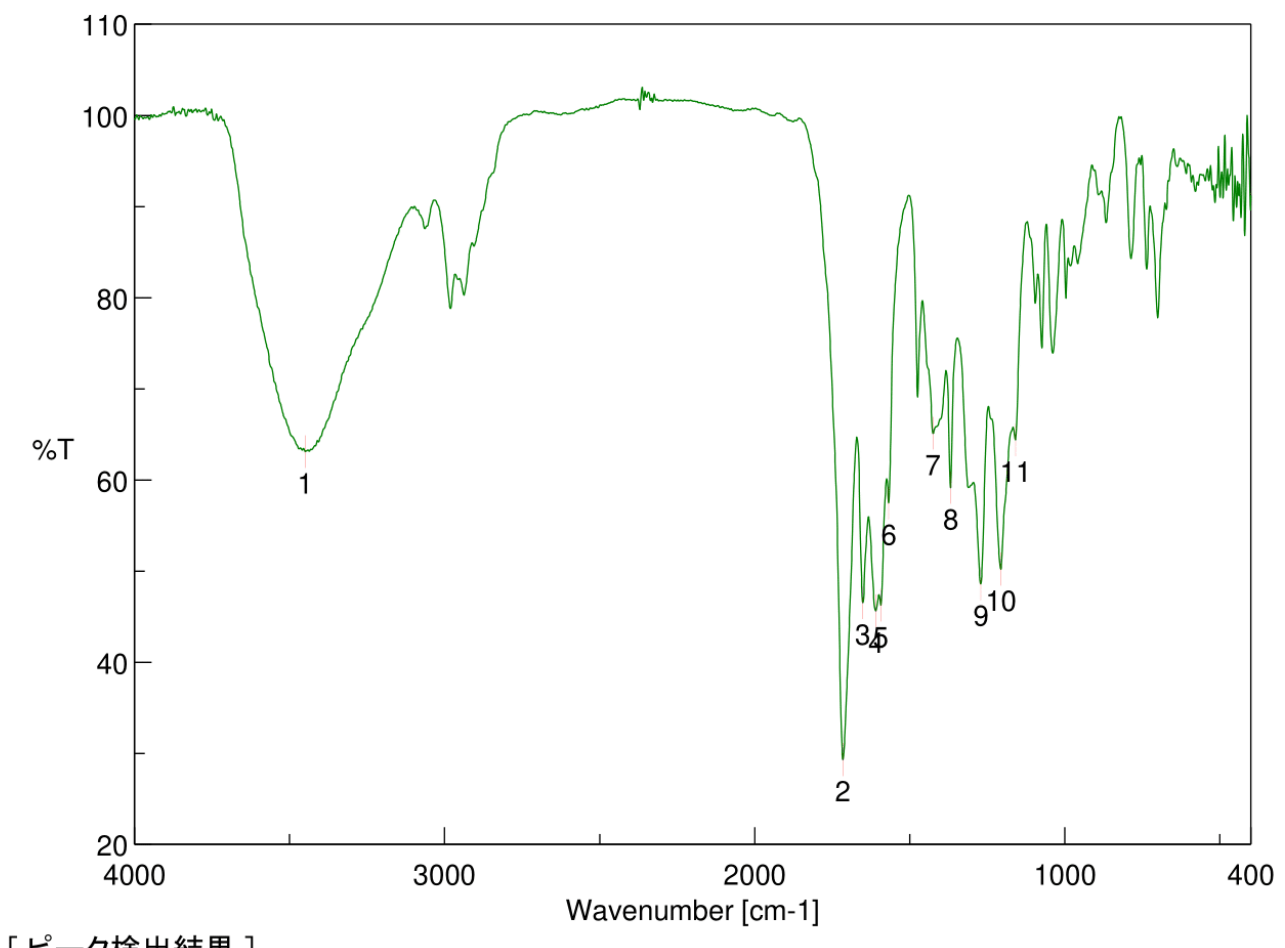

No. 位置強度

$2 \quad 1715.37 \quad 29.2933$

$4 \quad 1609.31 \quad 45.6155$

$6 \quad 1567.84 \quad 57.4671$

$8 \quad 1368.25 \quad 59.127$

$10 \quad 1206.26 \quad 50.199$

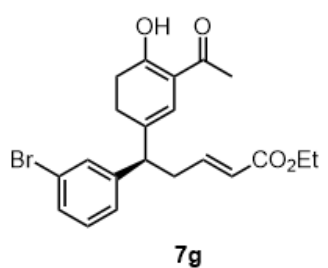




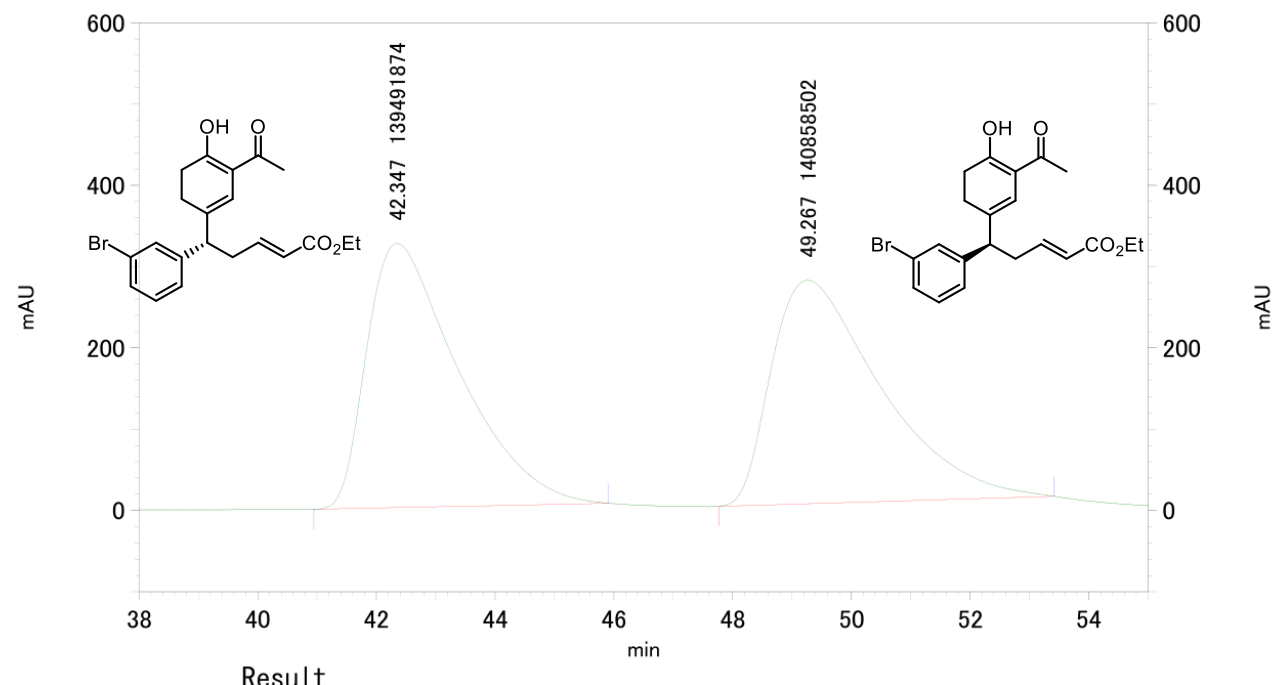

1: $210 \mathrm{~nm}, 4 \mathrm{~nm}$ 結果

Pk \# Retention time / min

42.347

Integration/\%

49. 267

50. 244

\begin{tabular}{|r|r|}
\hline$r-$ s & 100.000 \\
\hline
\end{tabular} 


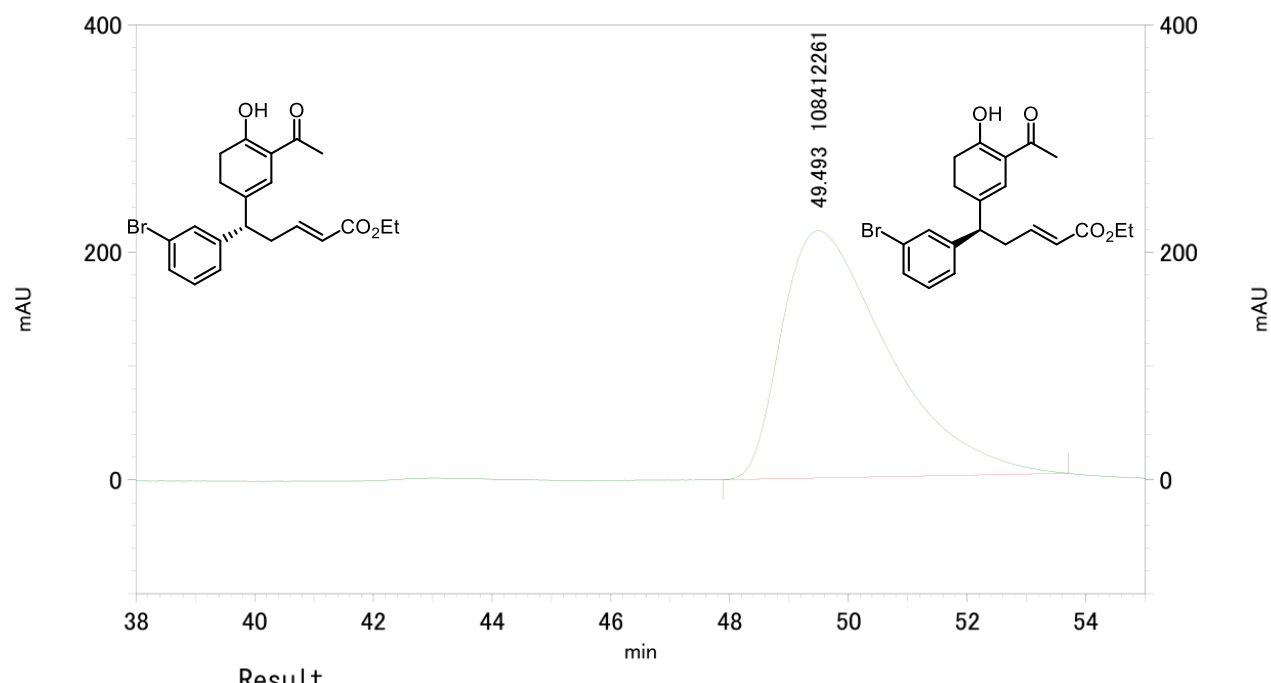

1: $210 \mathrm{~nm}, 4 \mathrm{~nm}$ 結果

$\mathrm{Pk} \#$ Retention time / min Integration/\% 49.493 100. 000

\begin{tabular}{r|r|r|}
\hline$r-$ 舛 & 100.000 \\
\hline Total &
\end{tabular}



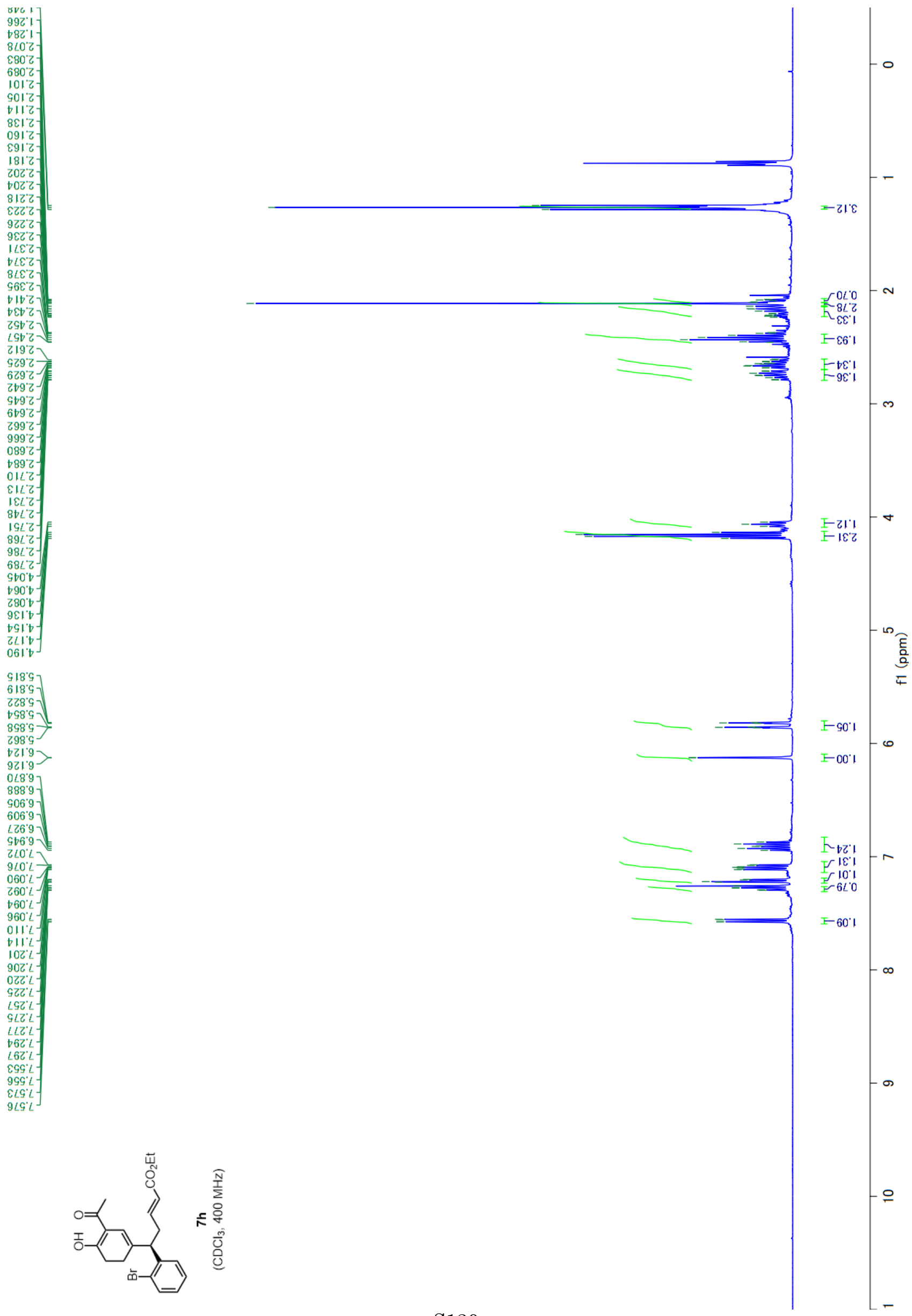
ह2เด1-

$\angle 961-$

tos

)
$08.98-$

عo $6 b-$

เะ.09-

$51801-$

$26.811-$

$\left.\begin{array}{l}96261 \\ 26.971\end{array}\right]$

$0 L$ LZI

127821

ต

૬टะเ

t) $101-$

sะ9 9 1-

0ะ 991 -

$168 L 1-$

ตะ $661-$

(1)

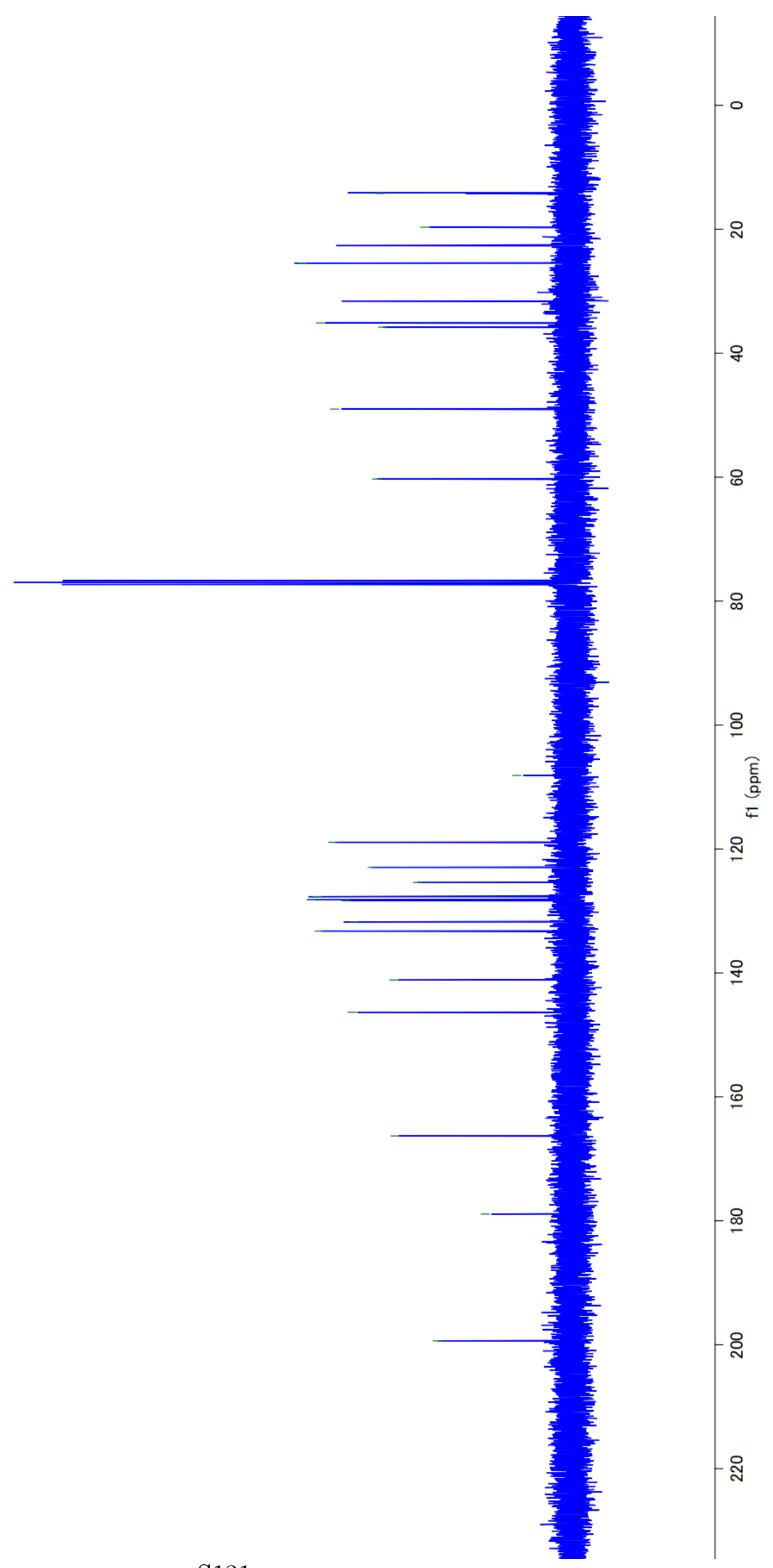

S121 


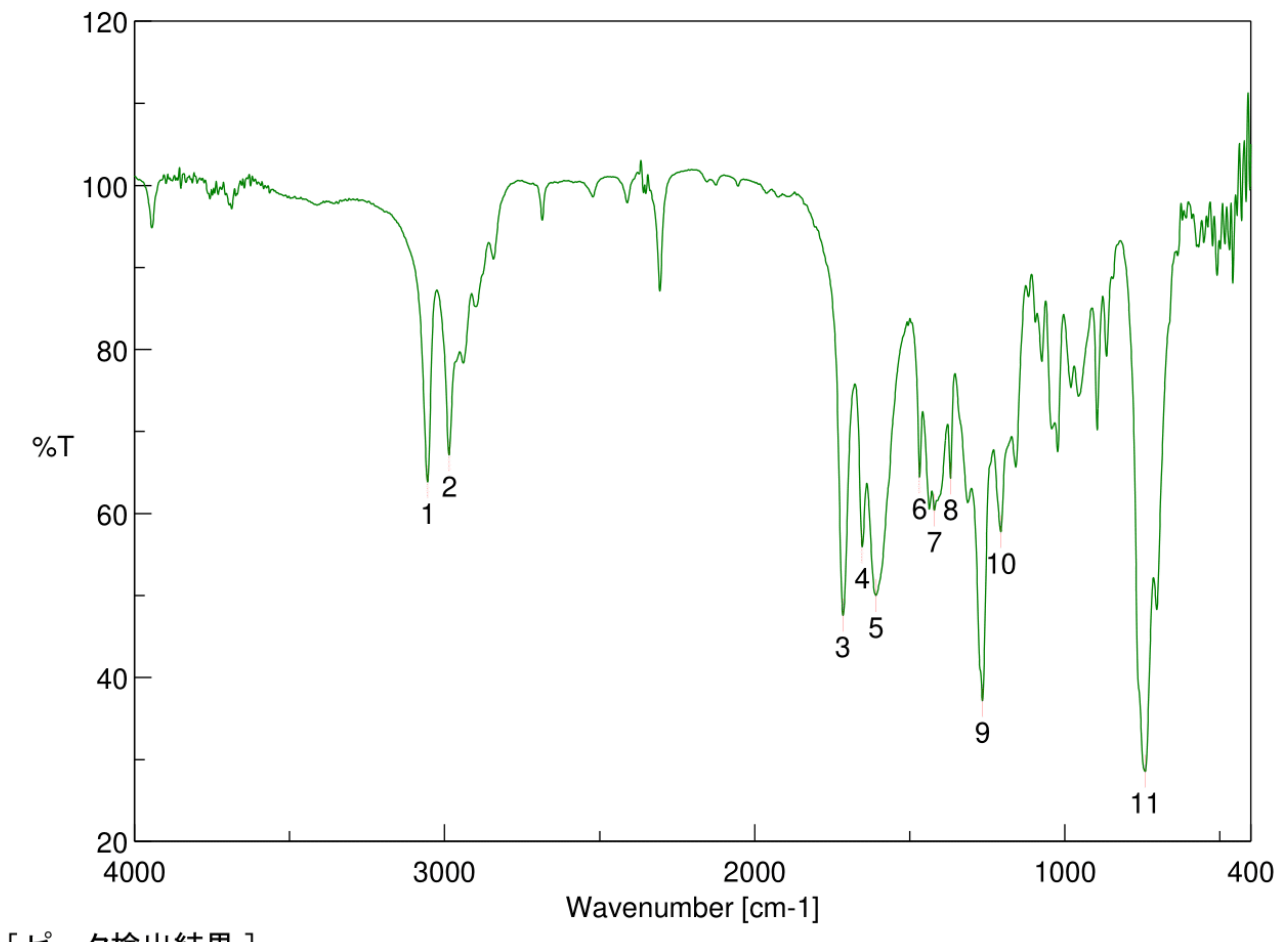

$\begin{array}{llllll}\text { [ ピーク検出結果 ] } & \text { l } & & & \\ \text { No. } & \text { 位置 } & \text { 強度 } & \text { No. } & \text { 位置 } & \text { 強度 } \\ 1 & 3054.69 & 63.8254 & 2 & 2985.27 & 67.1262 \\ 3 & 1715.37 & 47.5381 & 4 & 1653.66 & 55.9032 \\ 5 & 1609.31 & 49.9672 & 6 & 1468.53 & 64.4147 \\ 7 & 1420.32 & 60.3523 & 8 & 1368.25 & 64.2674 \\ 9 & 1265.07 & 37.1509 & 10 & 1206.26 & 57.7481\end{array}$

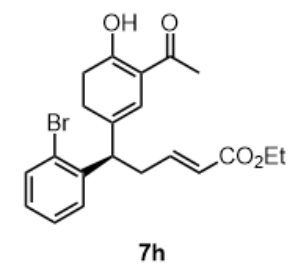




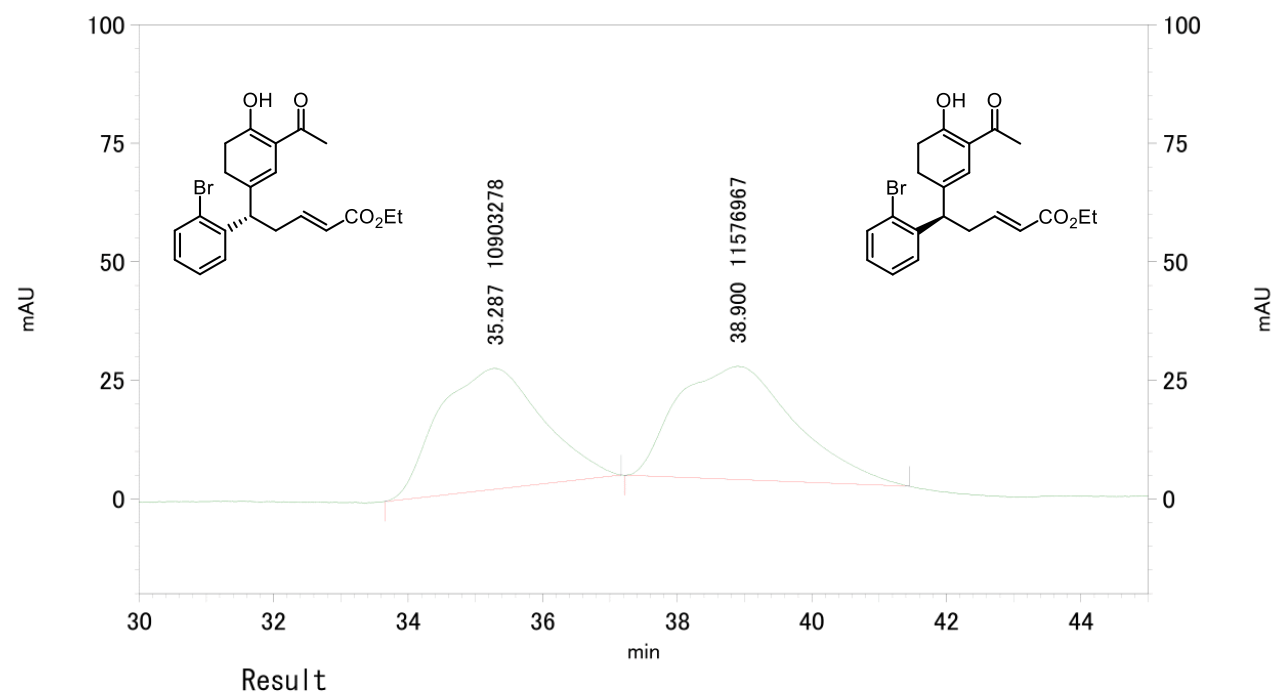

1:20 m m 4natse

$\mathrm{Pk} \# \quad$ Retention time / min

Integration/\%

35. 287

51. 498

\begin{tabular}{|r|r|}
\hline$r-$ 外 & 100.000 \\
\hline
\end{tabular} 


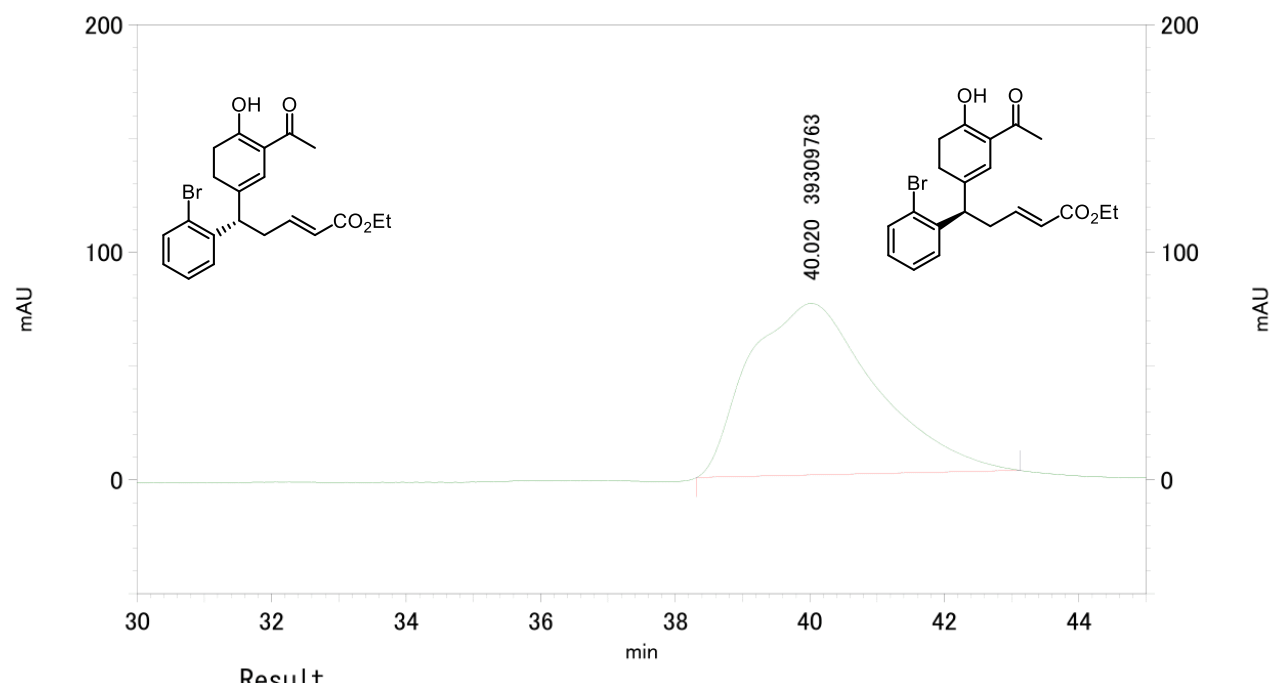

1: $210 \mathrm{~nm}, 4 \mathrm{~nm}$ 結果

Pk \# Retention time / min

Integration/\%

\begin{tabular}{r|r|}
\hline$r-$ 多 & 100.000 \\
\hline Total & \\
\hline
\end{tabular} 

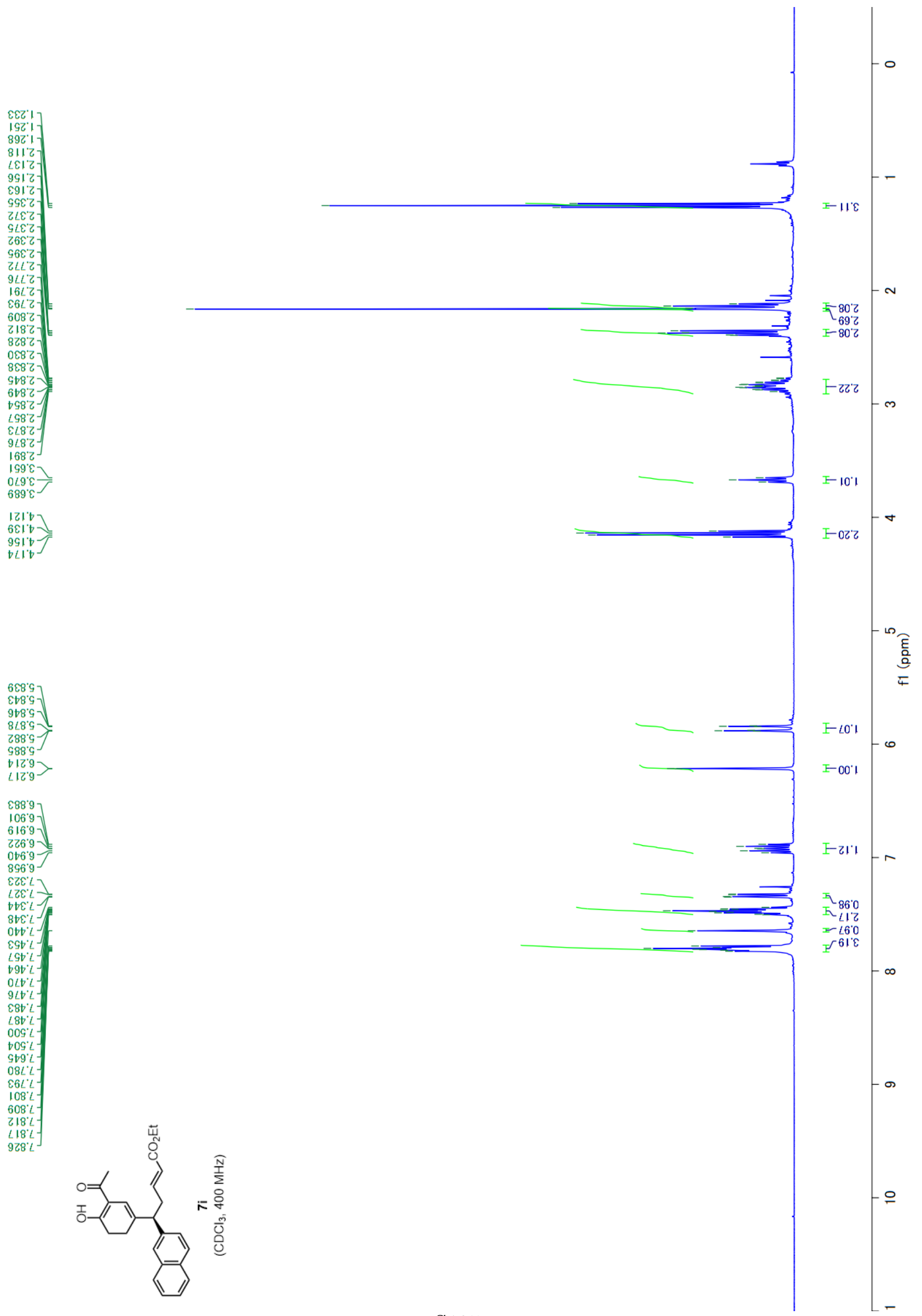


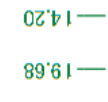

६६tbz-

$919 \varepsilon$
$99.9 \varepsilon$

$1119-$

9709-
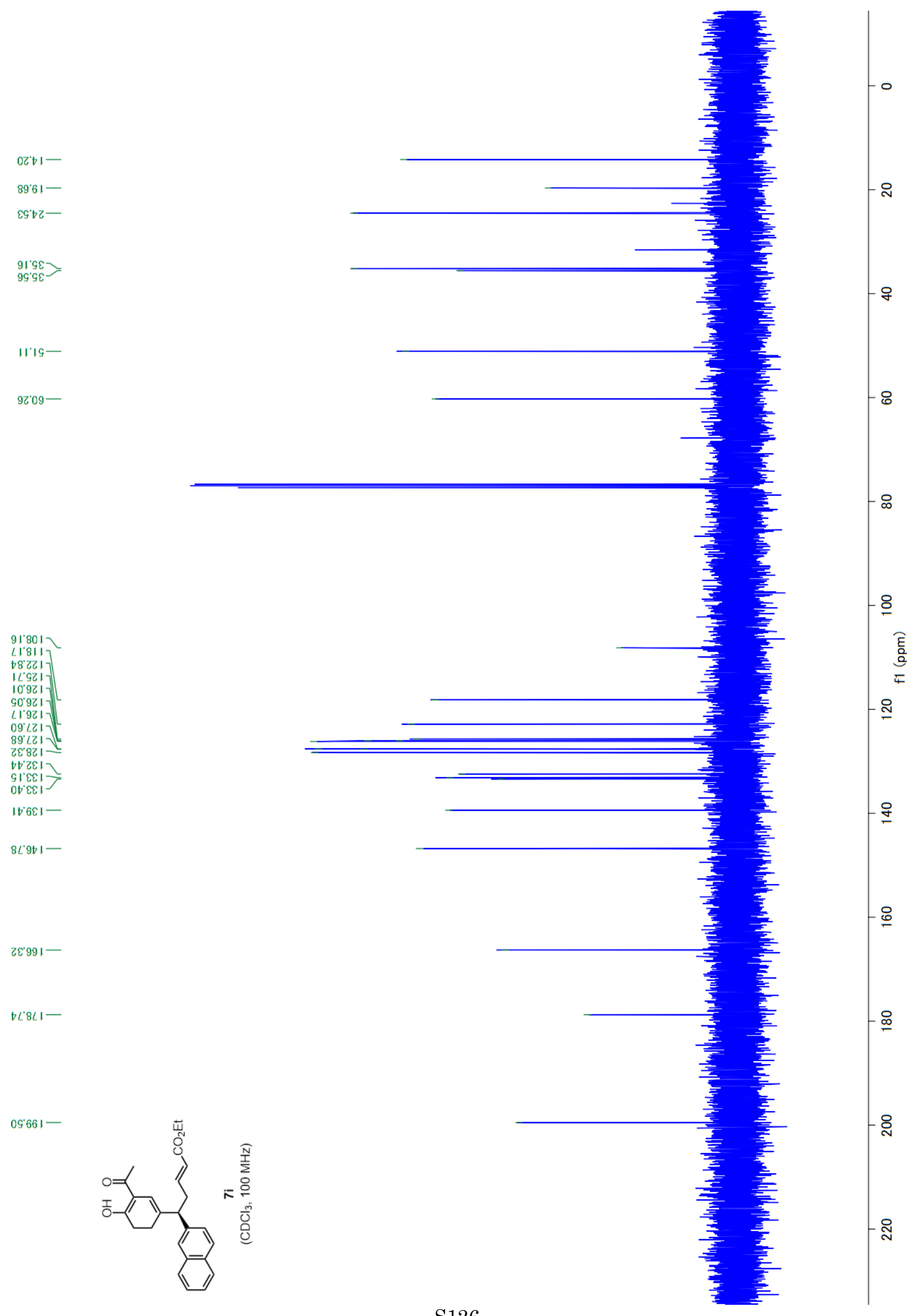

0966। -

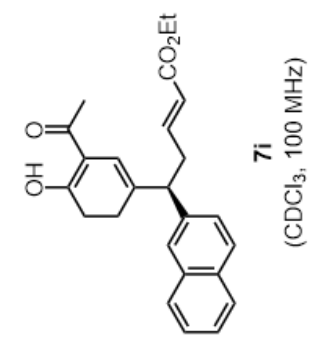




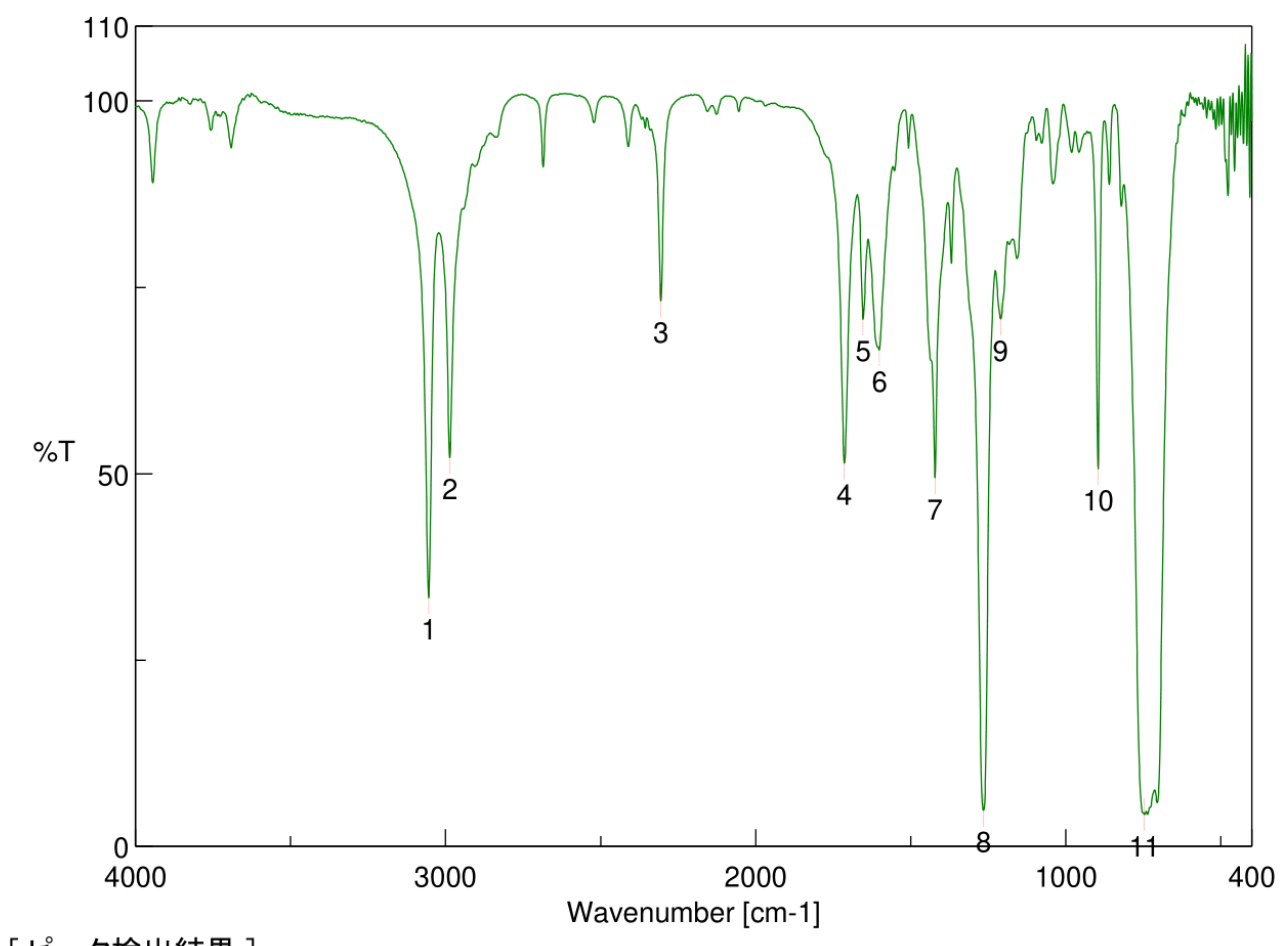

$\begin{array}{lll}\text { [ ピーク検出結果 ] } & \\ \text { No. } & \text { 位置 } & \text { 強度 } \\ 1 & 3054.69 & 33.3347 \\ 3 & 2305.48 & 73.1407 \\ 5 & 1653.66 & 70.6591 \\ 7 & 1421.28 & 49.4429 \\ 9 & 1210.11 & 70.7332 \\ 11 & 746.317 & 4.25432\end{array}$

No. 位置強度

$2 \quad 2986.23 \quad 52.1589$

$4 \quad 1714.41 \quad 51.3941$

$\begin{array}{lll}6 & 1601.59 & 66.5708\end{array}$

$\begin{array}{lll}8 & 1265.07 & 4.84804\end{array}$

$\begin{array}{lll}10 & 895.773 & 50.6384\end{array}$

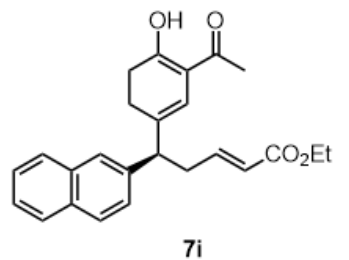




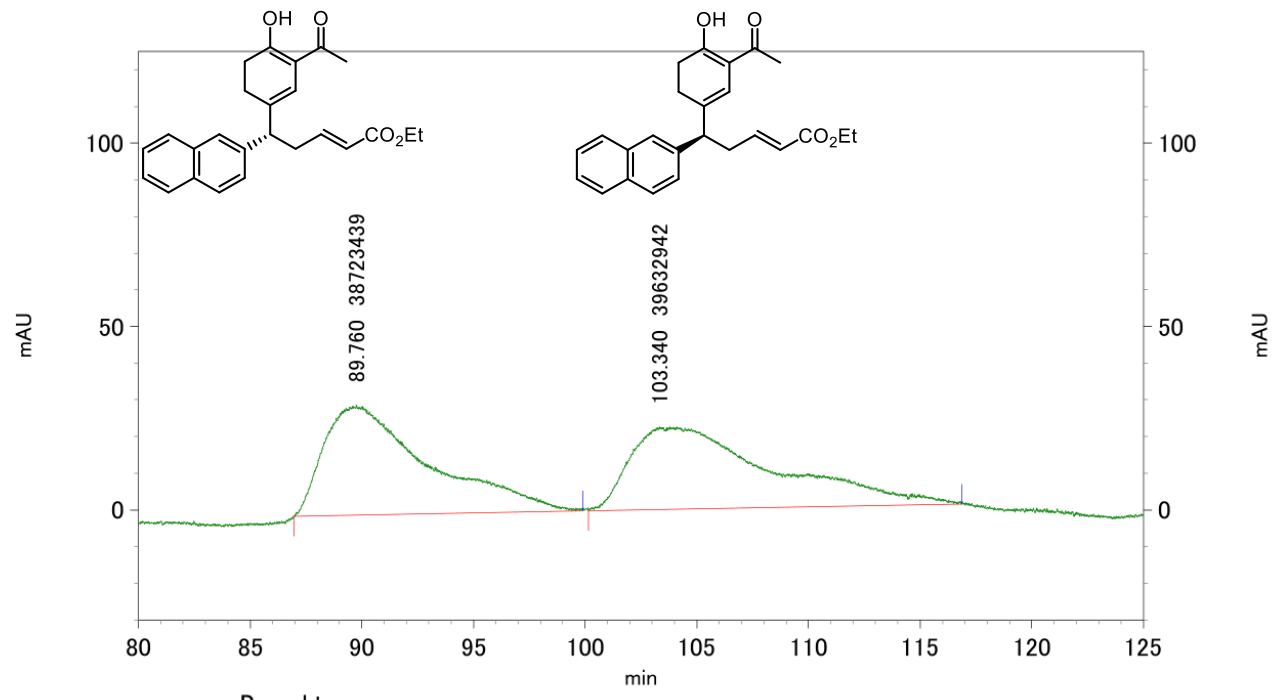

5: $210 \mathrm{~nm}, 4$ nm結果

Pk \# Retention time / min

Integration/ \%

89.760

50.580

\begin{tabular}{|r|r|}
\hline$r-$ 外 & 100.000 \\
\hline
\end{tabular} 


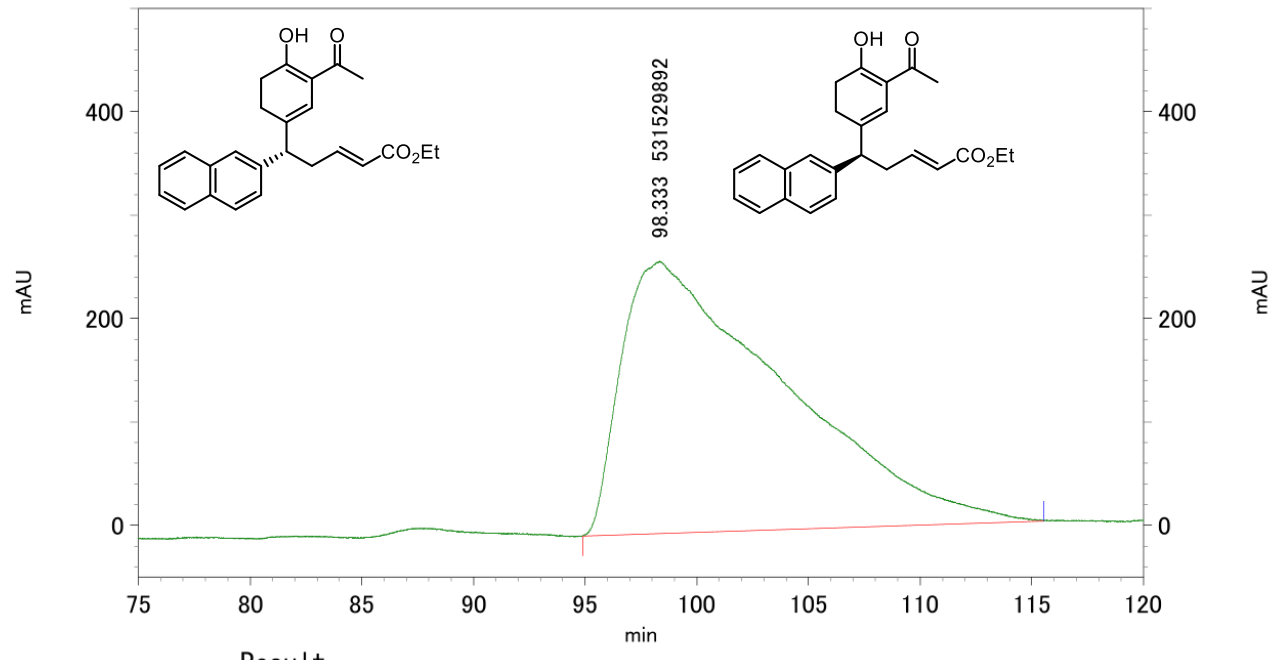

5: $210 \mathrm{~nm}, 4$ nm結果

Pk \# Retention time / min

Integration/\%

98. 333

100. 000

\begin{tabular}{|r|r|r|}
\hline$r-$ 㚈 & 100.000 \\
\hline Total & & 100
\end{tabular} 


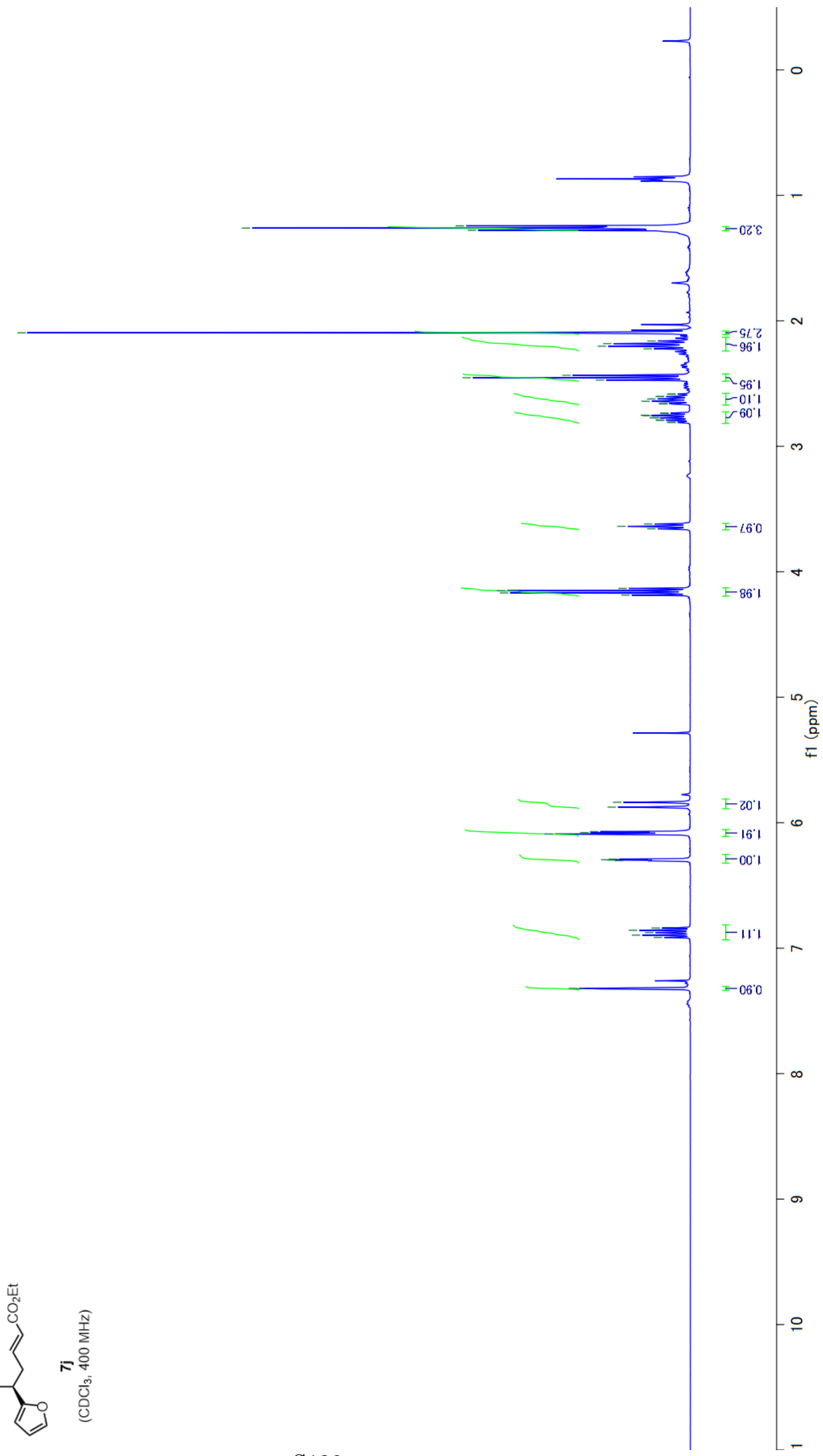




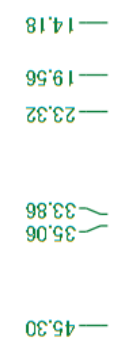

9209-

$88.901-$

El011-

L9.611-

10६६।-

$89^{\circ} 081-$

$89^{\prime}|t|-$

t6st-

Lて૬૬।-

$12991-$

ะ8 $841-$

6ะ $661-$

$$
\text { 球㝇 }
$$

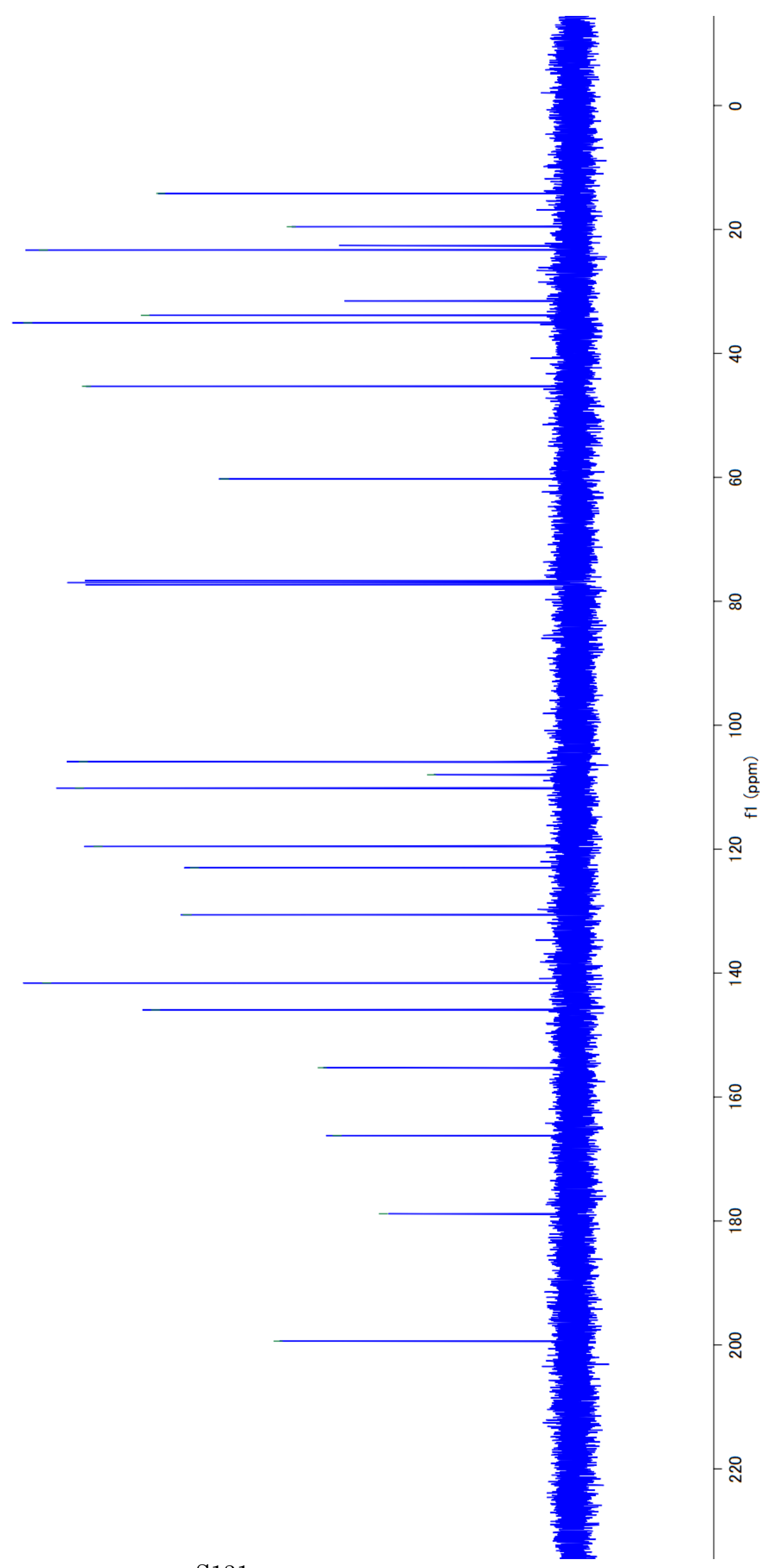

S131 


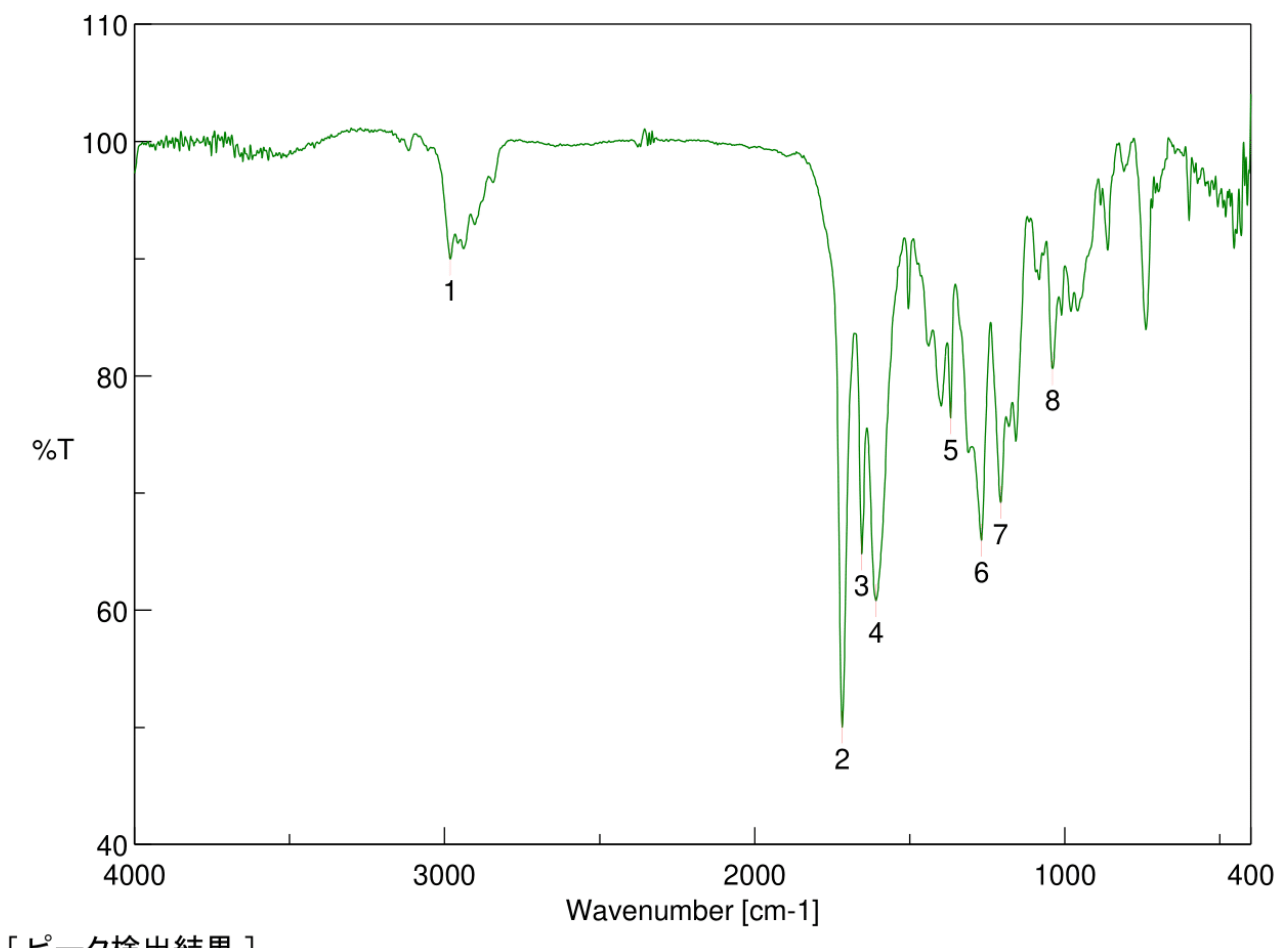

\begin{tabular}{|c|c|c|c|c|c|}
\hline \multicolumn{6}{|c|}{ [ピーク検出結果 ] } \\
\hline No. & 位置 & 強度 & No. & 位置 & 強度 \\
\hline 1 & 2981.41 & 89.9559 & 2 & 1717.3 & 49.9885 \\
\hline 3 & 1654.62 & 64.7977 & 4 & 1609.31 & 60.7895 \\
\hline & 1368.25 & 76.388 & 6 & 1268.93 & 65.96 \\
\hline & 1207.22 & 69.1995 & 8 & 1039.44 & 80.597 \\
\hline
\end{tabular}

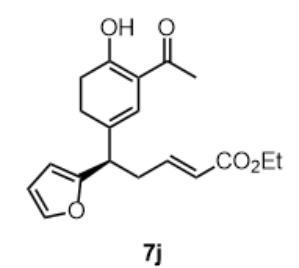




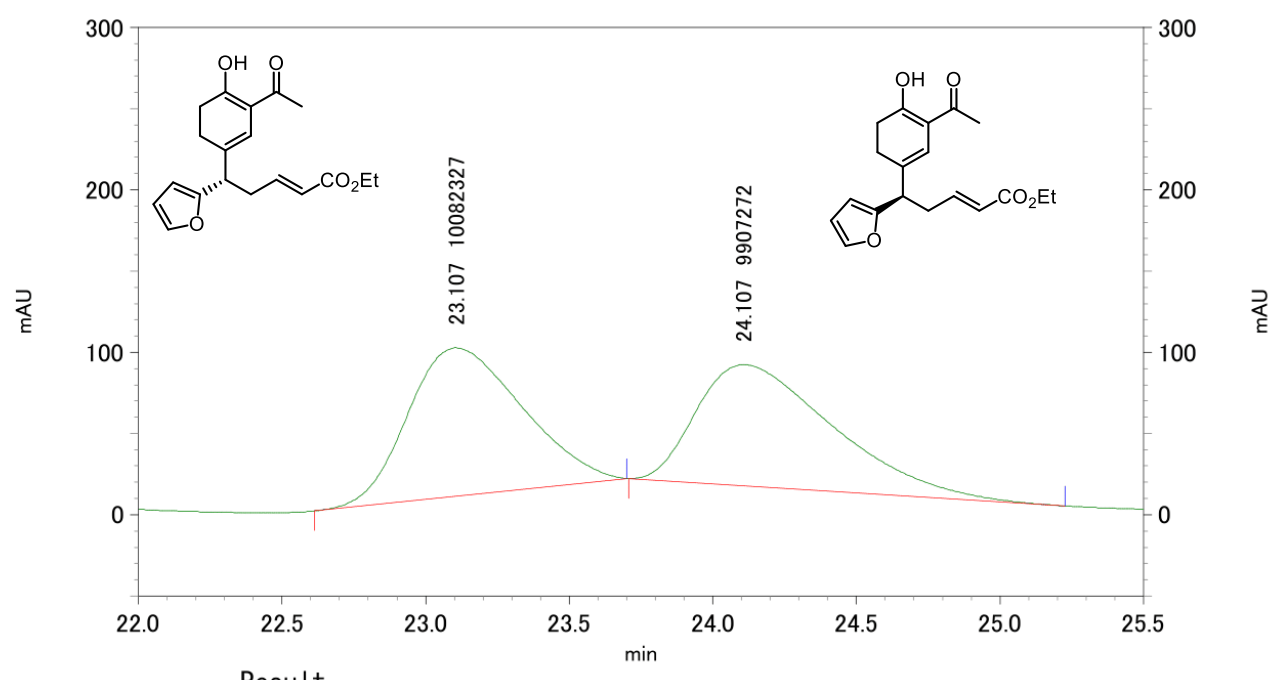

1: $319 \mathrm{~nm}, 4 \mathrm{~nm}$ 結果

Pk \# Retention time / min

Integration/ \%

50. 438

\begin{tabular}{|r|r|}
\hline$r-$ 舛 & 100.000 \\
\hline Total & \\
\hline
\end{tabular} 


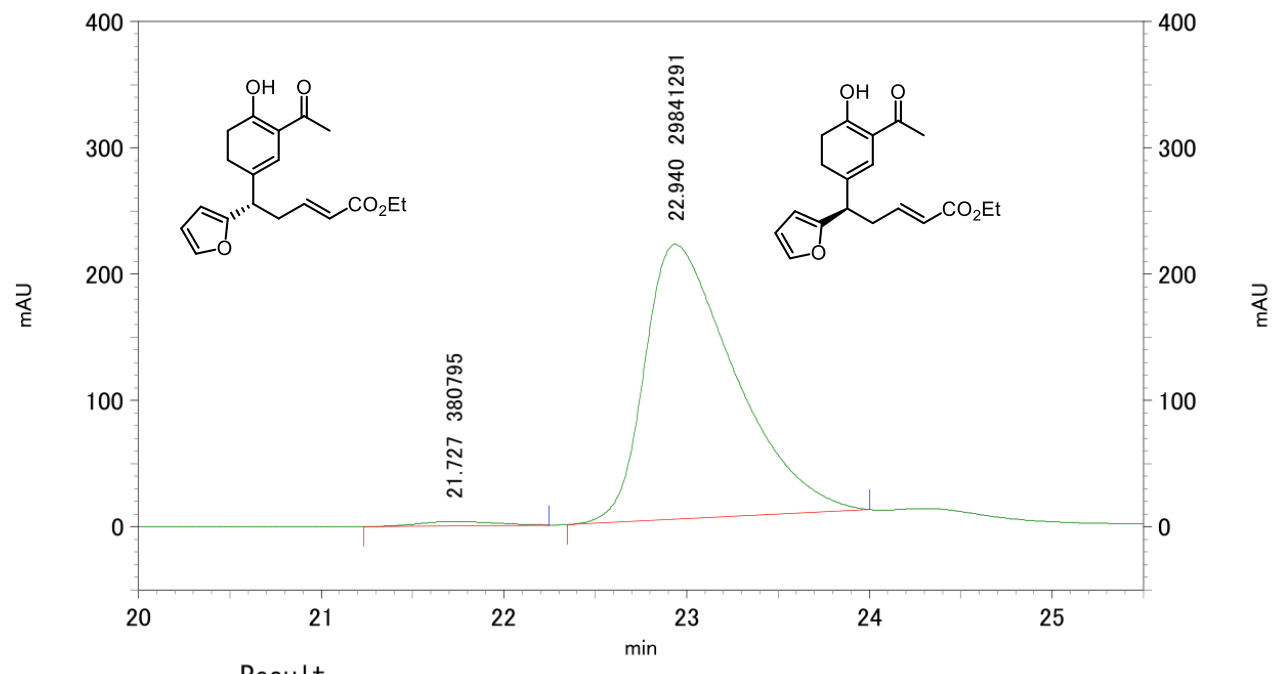

1: $319 \mathrm{~nm}, 4 \mathrm{~nm}$ 結果

Pk \# Retention time / min

Integration/\%

21.727

98. 740

\begin{tabular}{|r|r|}
\hline$r-$ 舛 & 100.000 \\
\hline
\end{tabular} 
IZ2'।

6EZ'।-

698 i-

LLZ'

$860^{\prime} z$

$\varepsilon 6 \mathrm{Z}-7$

$91 \varepsilon ; 2$

เहย'

$09 b^{\circ} \mathrm{C}$

ZLCZ

$06 b^{\prime} z$

Z6t'

8096

乙ह६'

द्c'?

$80 t 2]$

$\varepsilon Z L Z]$

$10 i z]$

to $i z]$

$\left.\begin{array}{l}0 t \\ 09 L \\ 0\end{array}\right]$

ع $9 L^{\circ} \mathrm{z}$

$18 t^{\circ} \mathrm{J}$

๑ाट

ยะช

021 th

हEl

ISI.

EsI't

9sib

691 t

$1,1, t$

$b 115$
L815

${ }^{888^{\circ} 9}=$

8119

$\left.66 L^{\circ} 9\right]$

$058^{\circ}-$

हะ8. 9

$198^{\circ} 9$

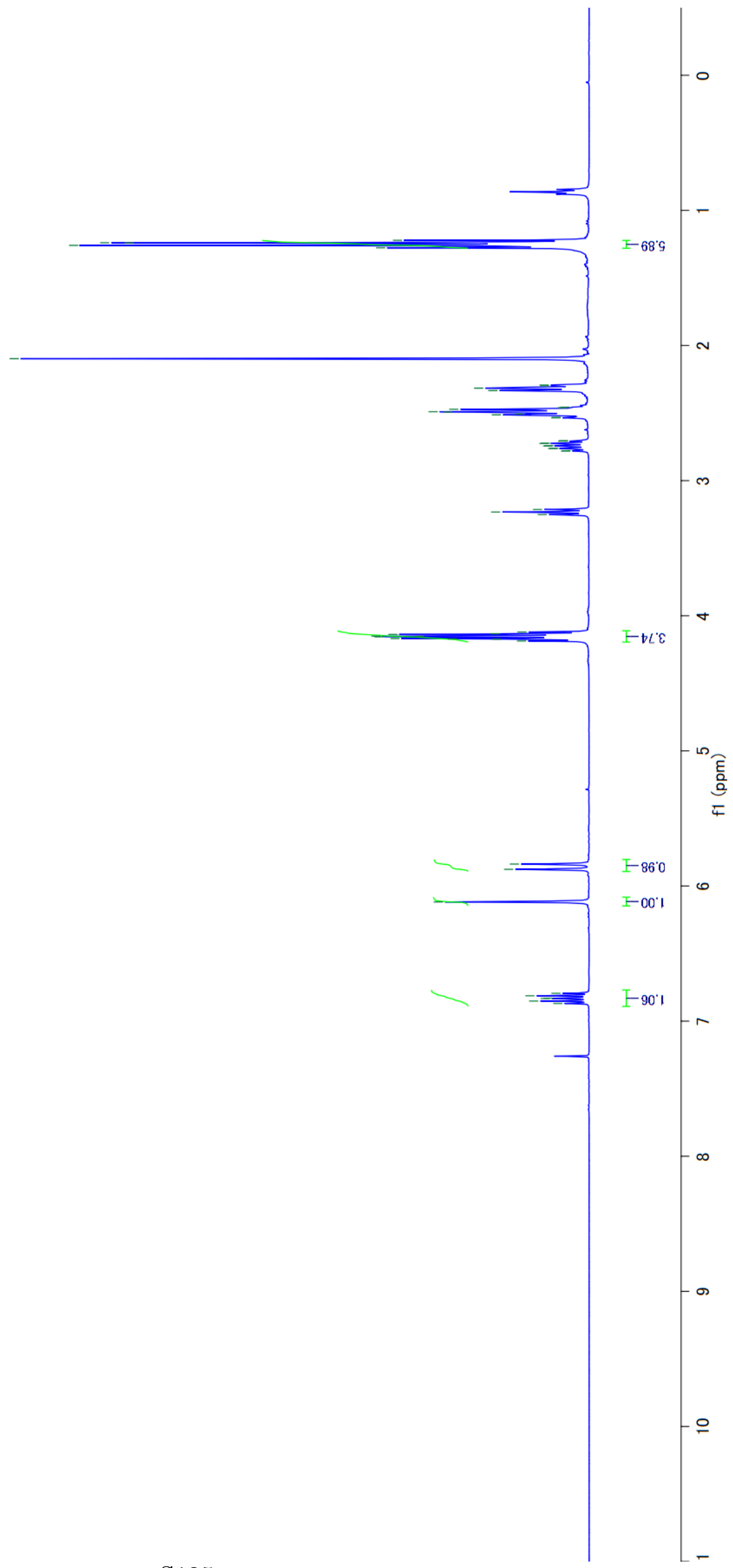


$91+11$
6101

8961 -

ต9ะ乙-

1Lวย-

$88^{\prime \prime 19-~}$

1809
00.19

S6: $201-$

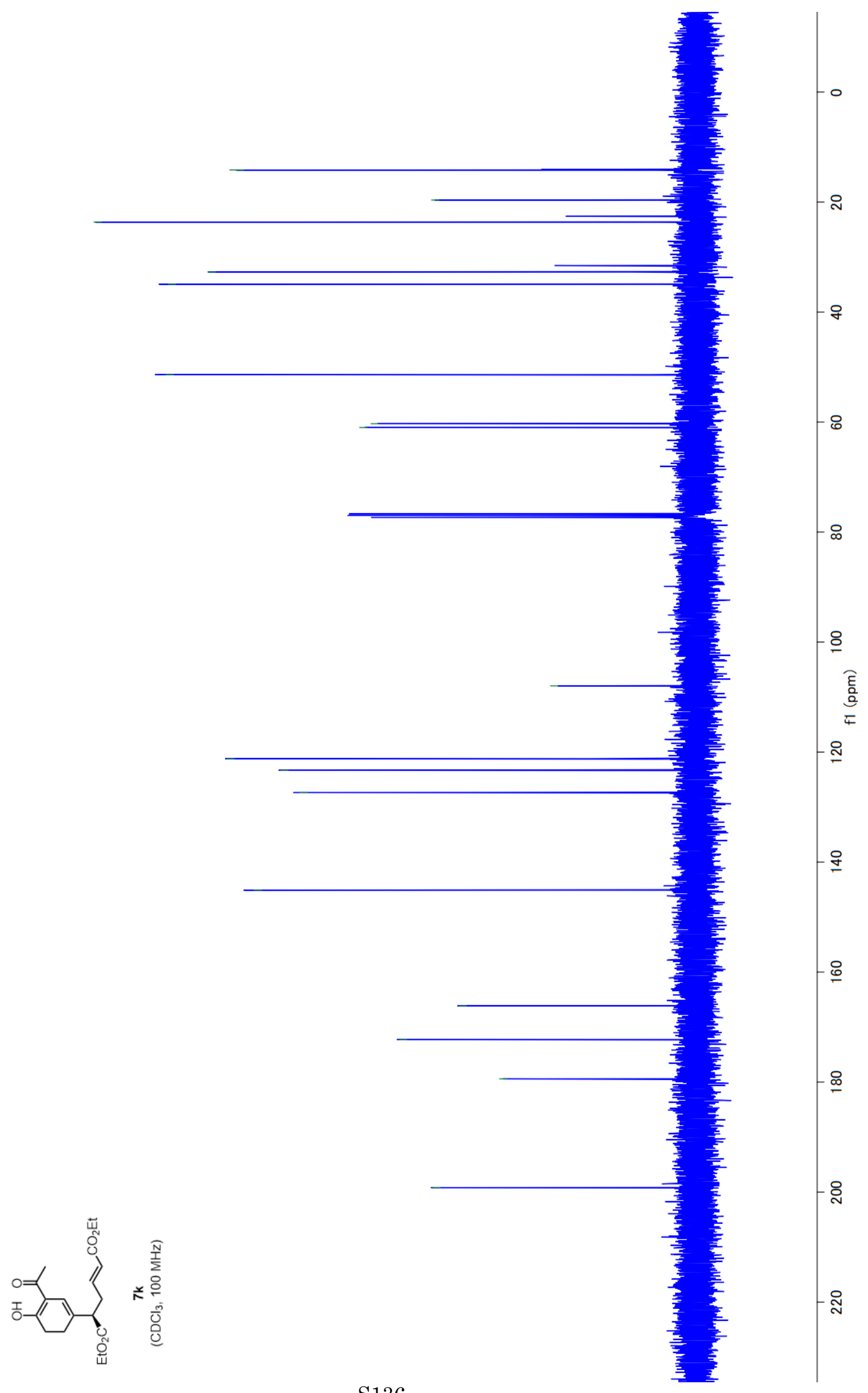

$96201-$

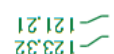

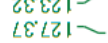

$01961-$

S136 


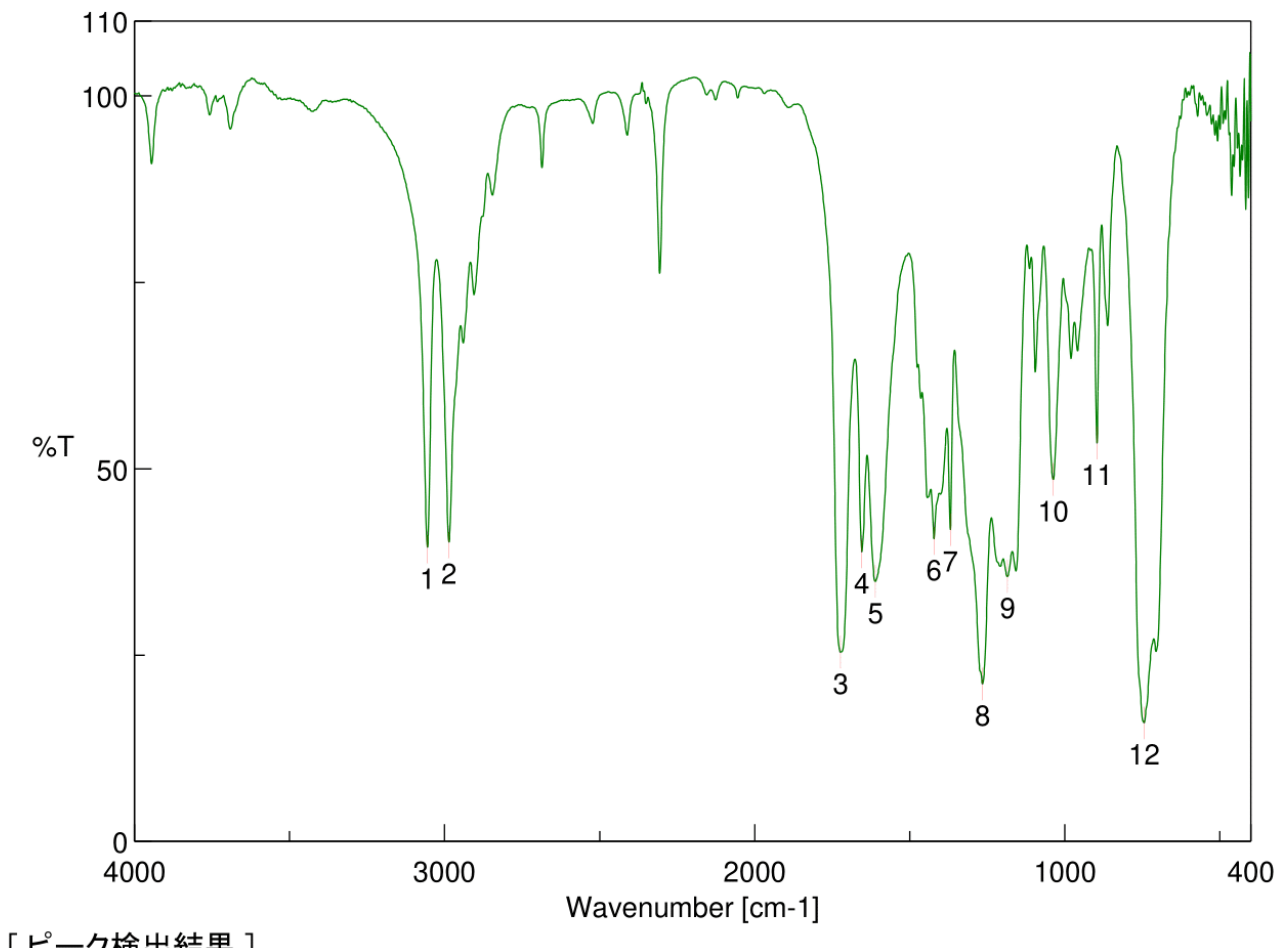

\begin{tabular}{llllll}
\multicolumn{2}{l}{ [ピーク検出結果 ] } & & & & \\
No. & 位置 & 強度 & No. & 位置 & 強度 \\
1 & 3055.66 & 39.4771 & 2 & 2986.23 & 40.1312 \\
3 & 1723.09 & 25.3759 & 4 & 1654.62 & 38.796 \\
5 & 1611.23 & 34.8732 & 6 & 1421.28 & 40.6249 \\
7 & 1369.21 & 41.7892 & 8 & 1265.07 & 21.0947 \\
9 & 1185.04 & 35.5215 & 10 & 1037.52 & 48.5114 \\
11 & 896.737 & 53.4239 & 12 & 744.388 & 15.907
\end{tabular}

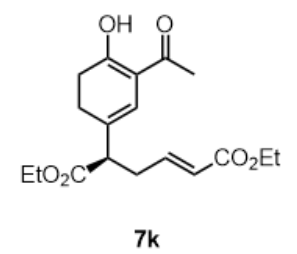




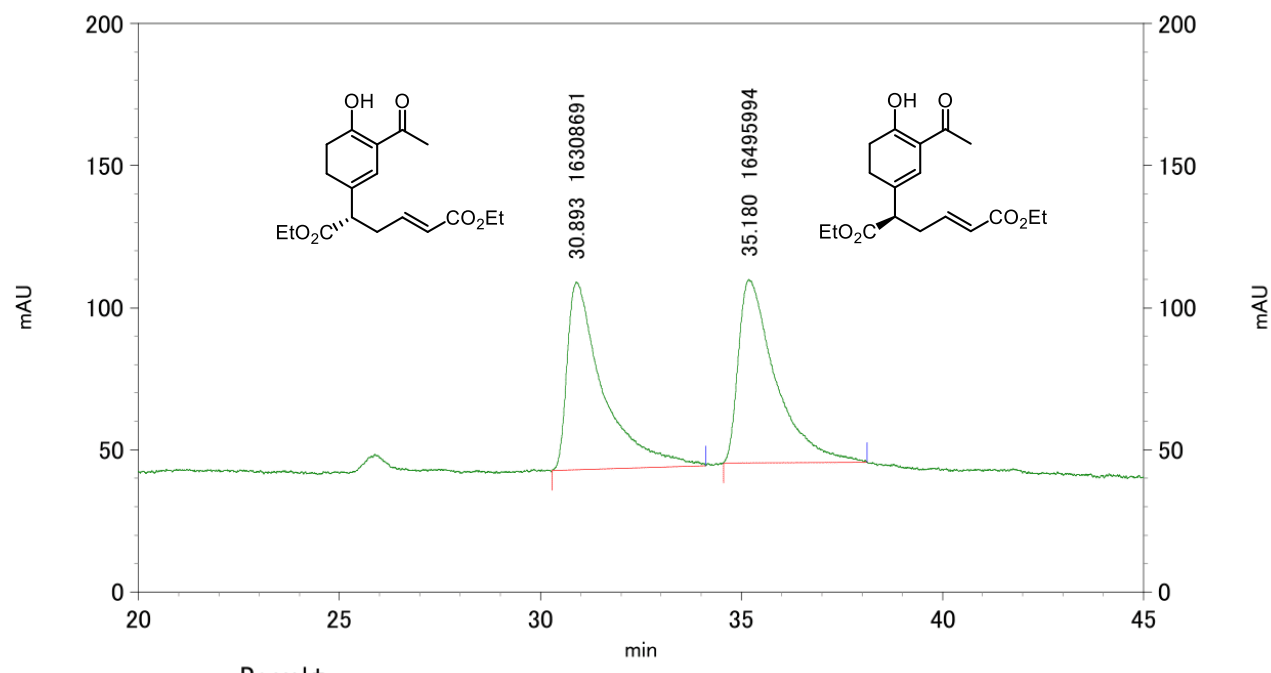

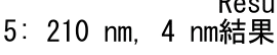

Pk \# Retention time / min

Integration/ \%

30.893

\begin{tabular}{l|l} 
r-夕夕 & \\
otal &
\end{tabular}

100. 000 


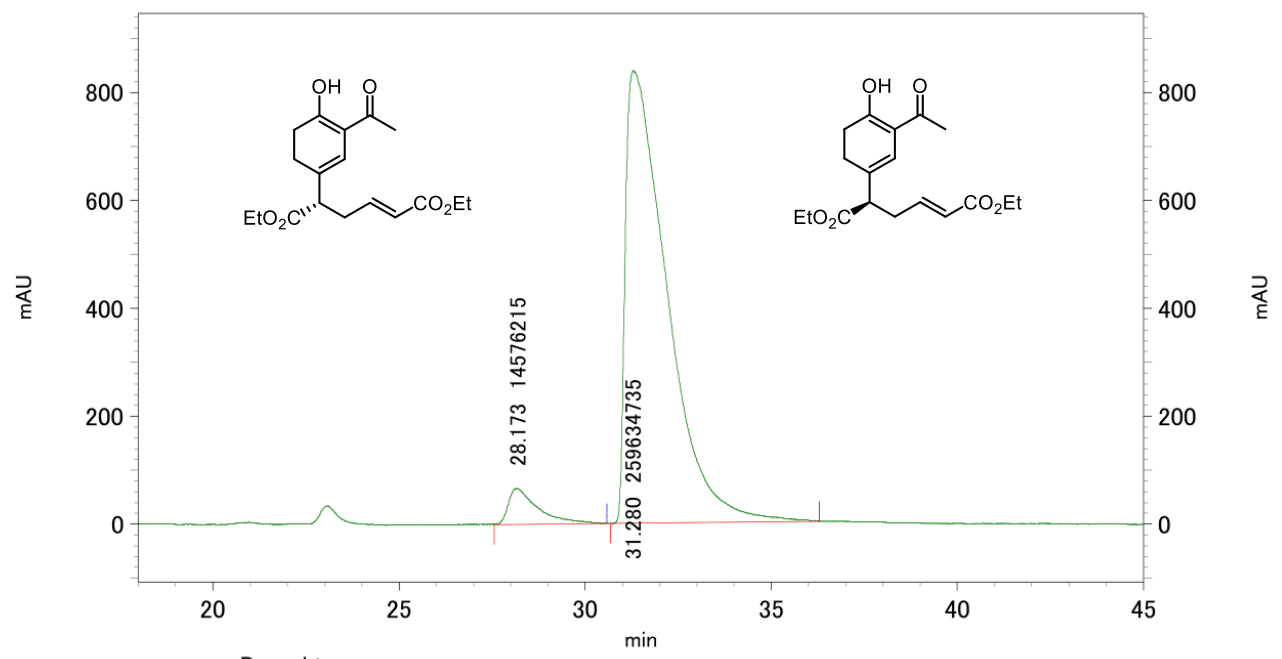

5: $210 \mathrm{~nm}, 4 \mathrm{~nm}$ 結果

Pk \# Retention time / min

Integration/\%

28.173

94.684

\begin{tabular}{|r|r|}
\hline$r-$ 外 & 100.000 \\
\hline
\end{tabular} 


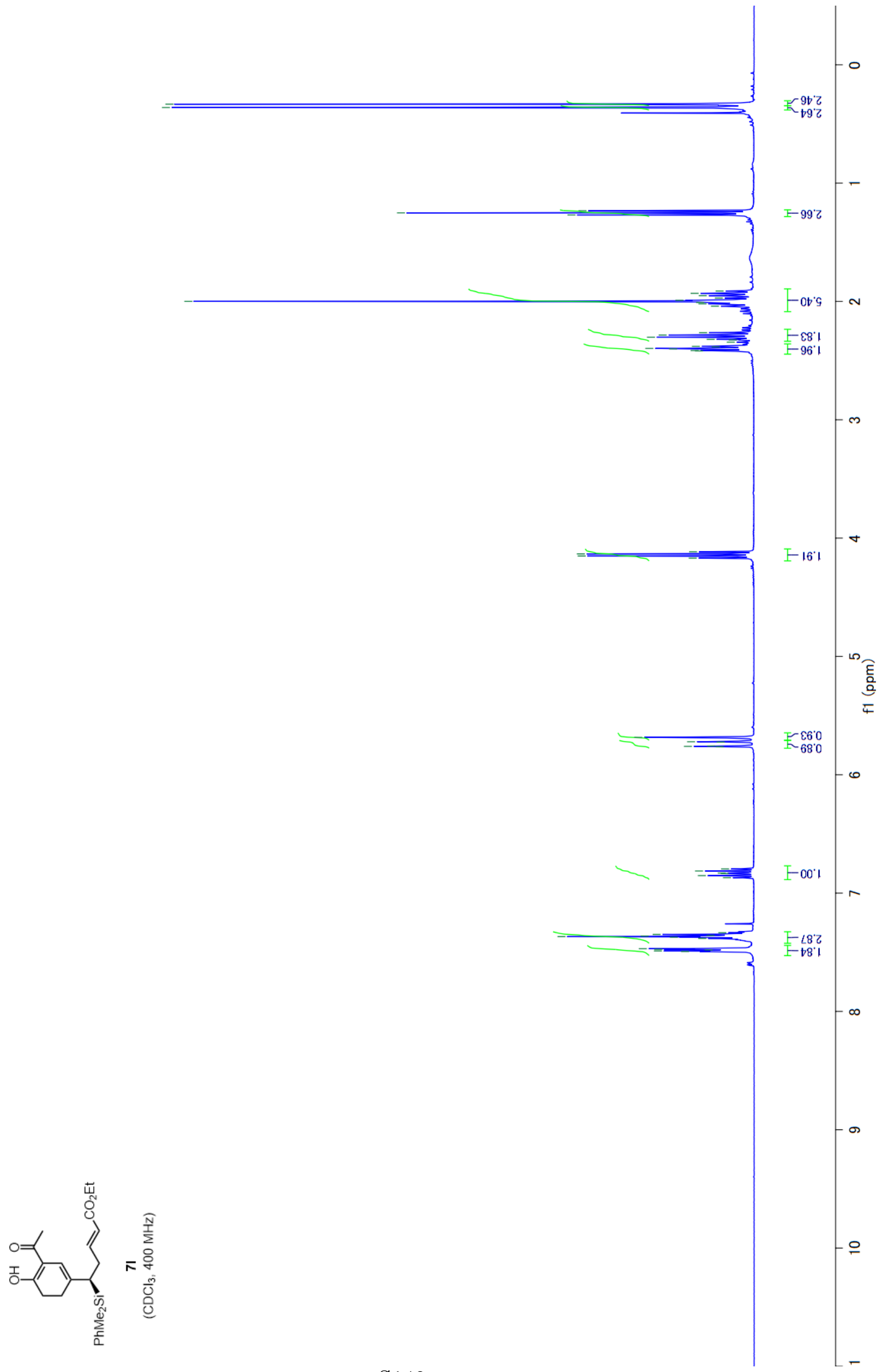




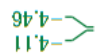

ZZ七

$96.92-$

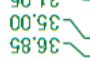

8109

$69^{\circ} 801-$

$06^{\circ} 911-$

乙9. -

$\varepsilon 8\lfloor z 1=$

ट9เ

$06.81-$
$16981-$

$06801-$

$\angle t 991-$

trLLI-

$6 \varepsilon^{\circ} 661-$

>

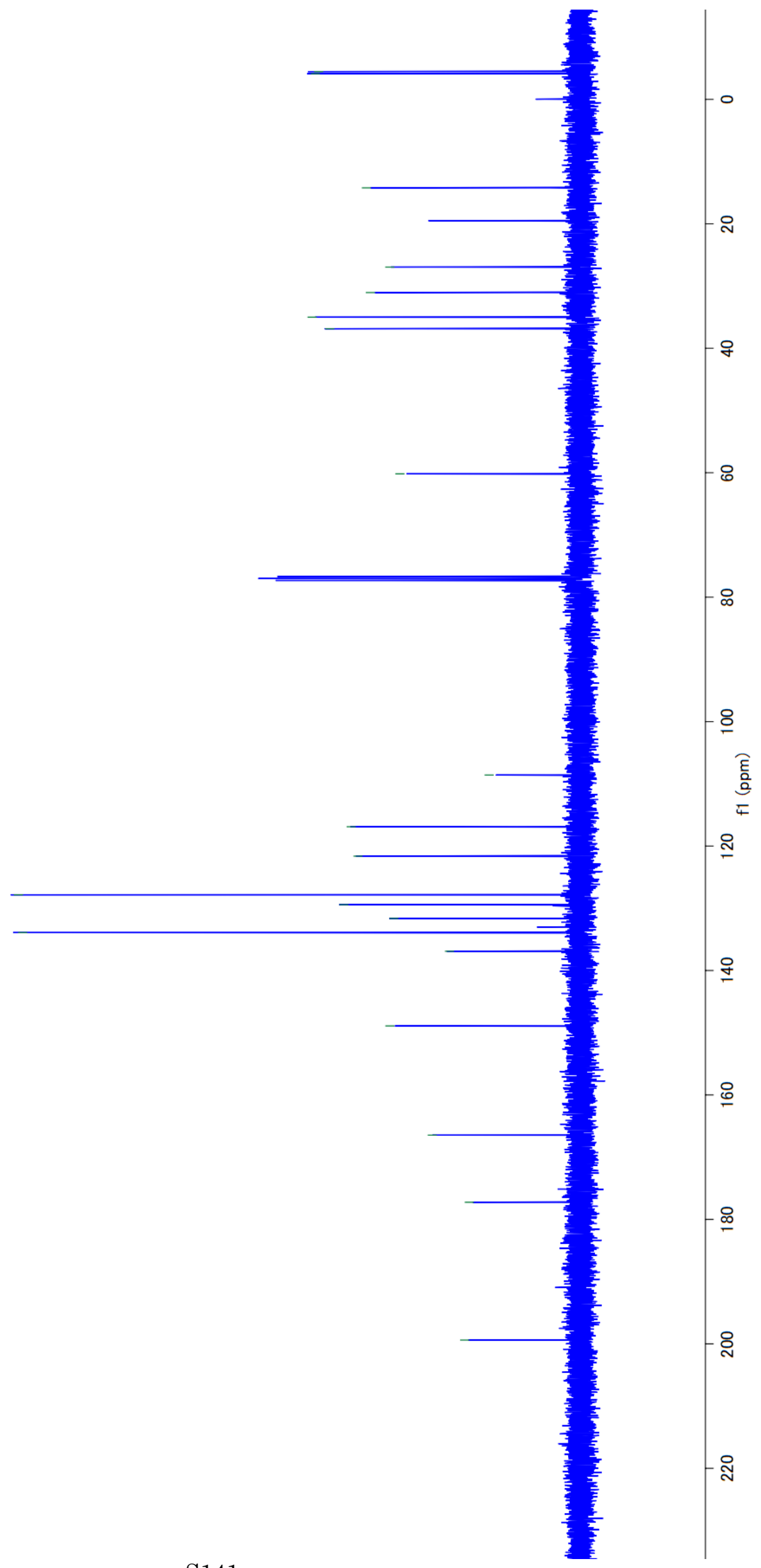




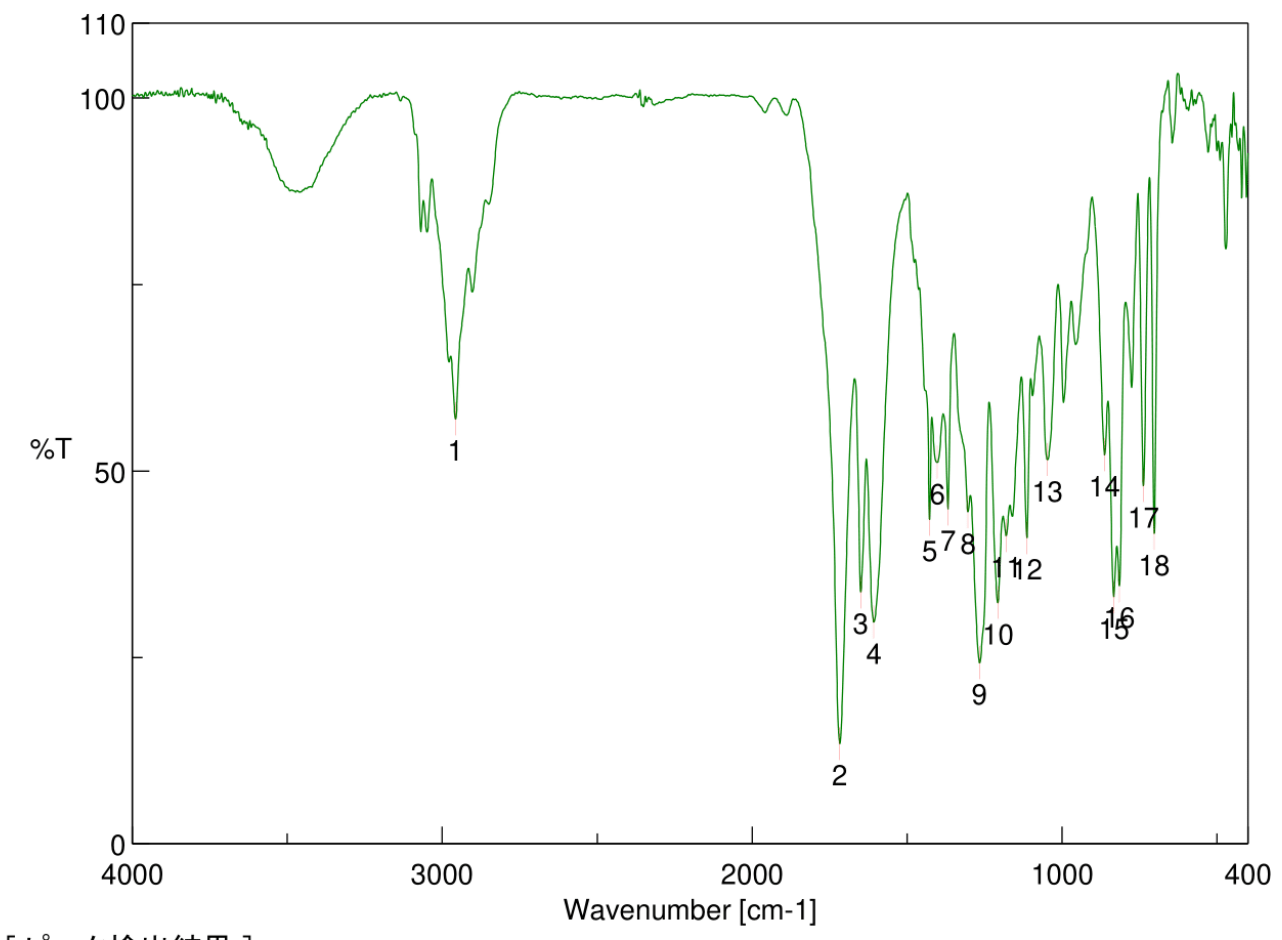

[ピーク検出結果 ]

$\begin{array}{lllllll}\text { No. } & \text { 位置 } & \text { 強度 } & \text { No. } & \text { 位置 } & \text { 強度 } \\ 1 & 2957.3 & 56.9459 & 2 & 1717.3 & 13.3922 & \\ 3 & 1649.8 & 33.7556 & 4 & 1607.38 & 29.6971 \\ 5 & 1427.07 & 43.4646 & 6 & 1402.96 & 51.1091 \\ 7 & 1368.25 & 44.8572 & 8 & 1303.64 & 44.5131 \\ 9 & 1266.04 & 24.2523 & 10 & 1207.22 & 32.2991 \\ 11 & 1180.22 & 41.2676 & 12 & 1113.69 & 41.0227 \\ 13 & 1047.16 & 51.4905 & 14 & 862.989 & 52.1535 & \\ 15 & 833.098 & 33.0918 & 16 & 814.777 & 34.5448 & \\ 17 & 737.639 & 47.9865 & 18 & 702.926 & 41.6157 & \end{array}$




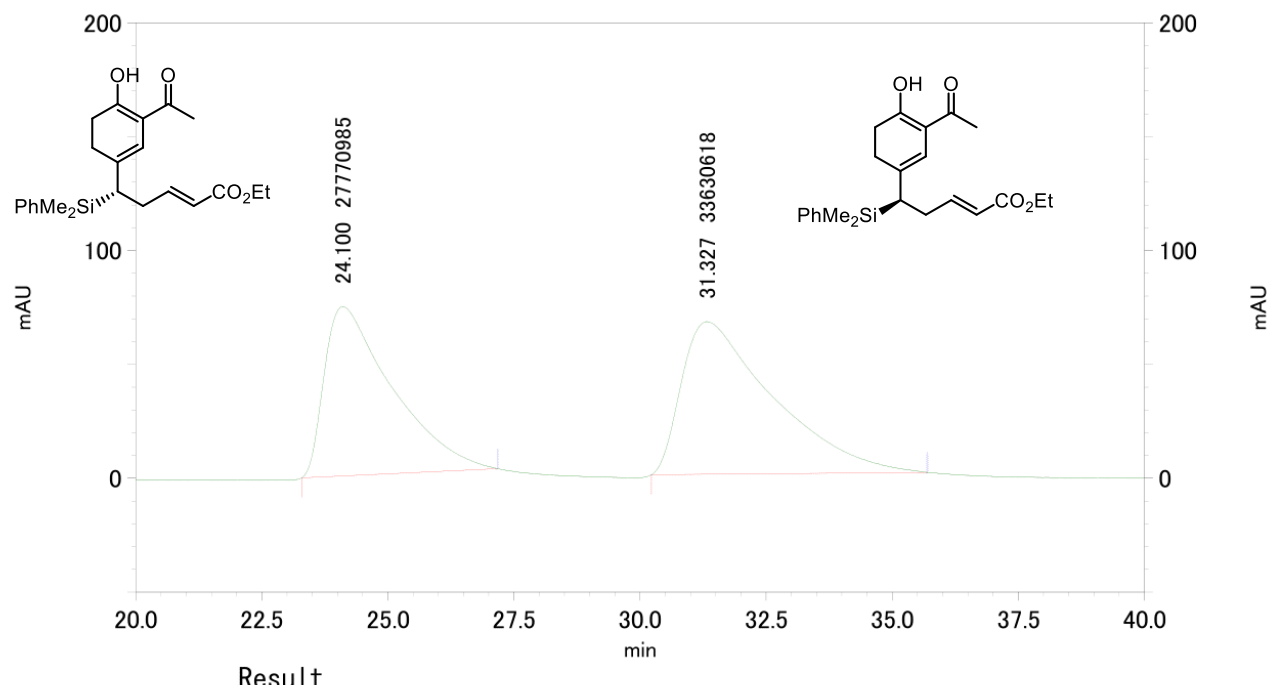

1: $211 \mathrm{~nm}, 4 \mathrm{~nm}$ 結果

Pk \# Retention time / min

Integration/\%

24. 100

54. 772

\begin{tabular}{|r|r|r|}
\hline$r-$ 外 & 100.000 \\
\hline Total & & 100
\end{tabular} 


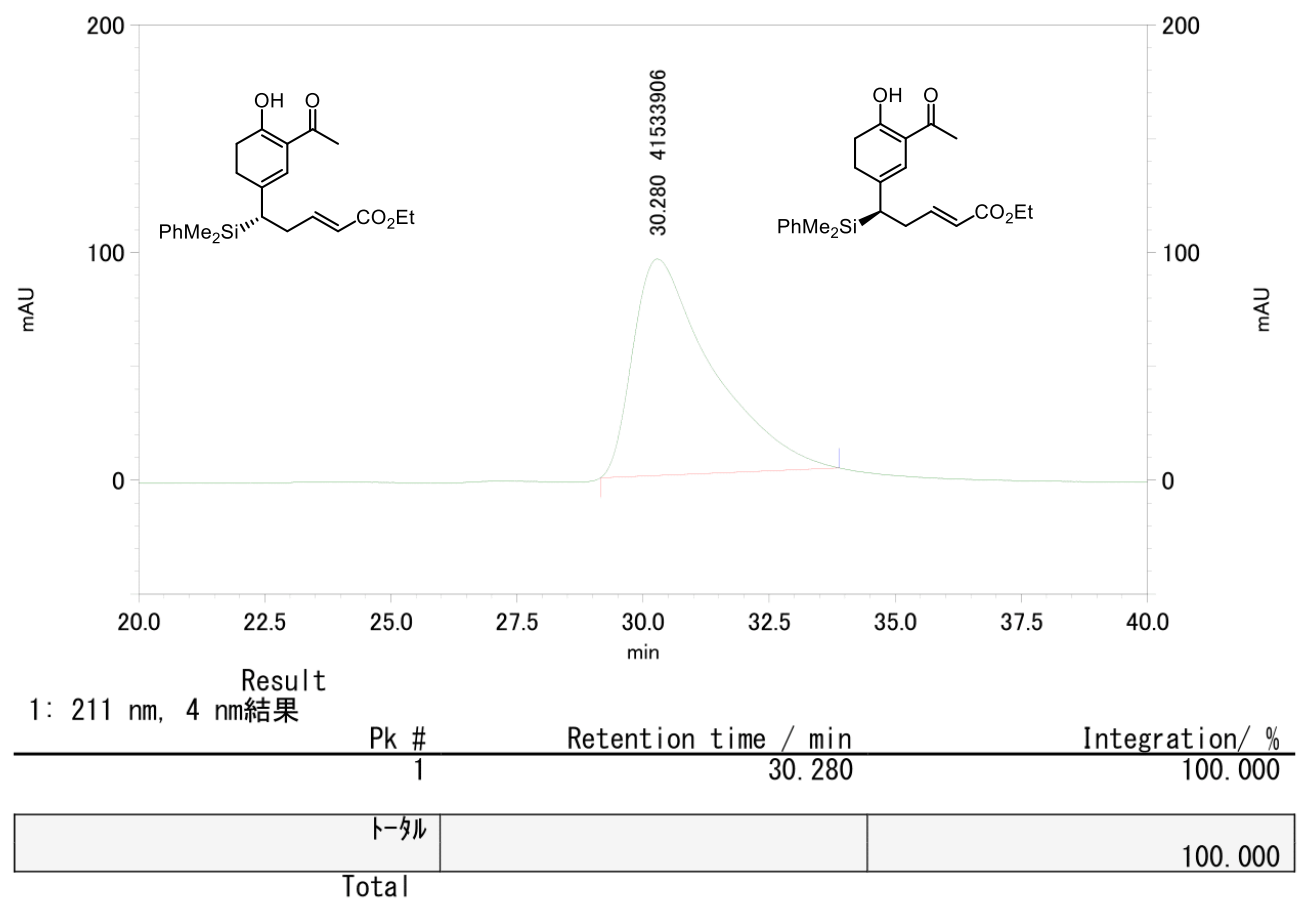


צ्Y० '

$10 \varepsilon^{\circ} 1$

द्वा?

981

ZZZ'

$102 ' z$

E9z

0962

$\varepsilon 9 b 7$

$18 \mathrm{~b}^{\circ} \mathrm{C}$

$88 \mathrm{~b}$

$969^{\prime} \mathrm{Z}$

$66 \mathrm{~s}^{2} \mathrm{z}$

$219 \mathrm{Z}$

$129^{\circ} \mathrm{z}$

हE9

$6+99^{\circ} \mathrm{C}$

$\varepsilon 99^{\circ} \mathrm{Z}$

$099^{\circ} \mathrm{Z}$

$\varepsilon 99^{\circ} \mathrm{Z}$

689

toL 2

$0 Z L ' Z$

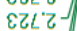

$6 \& L$

$680^{\circ} \varepsilon$

${ }^{6} 0^{\circ} \varepsilon$

8Z૬'

t91't

$681^{\circ}$

661 't

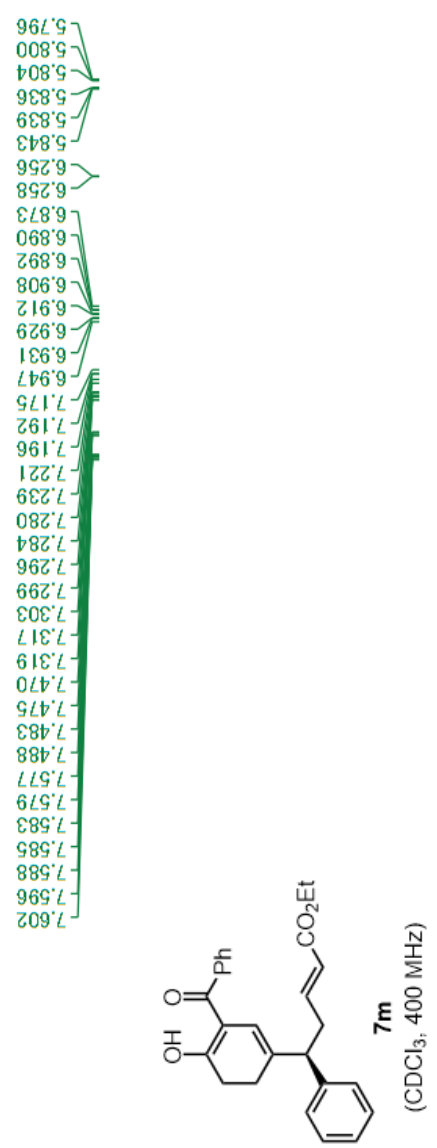

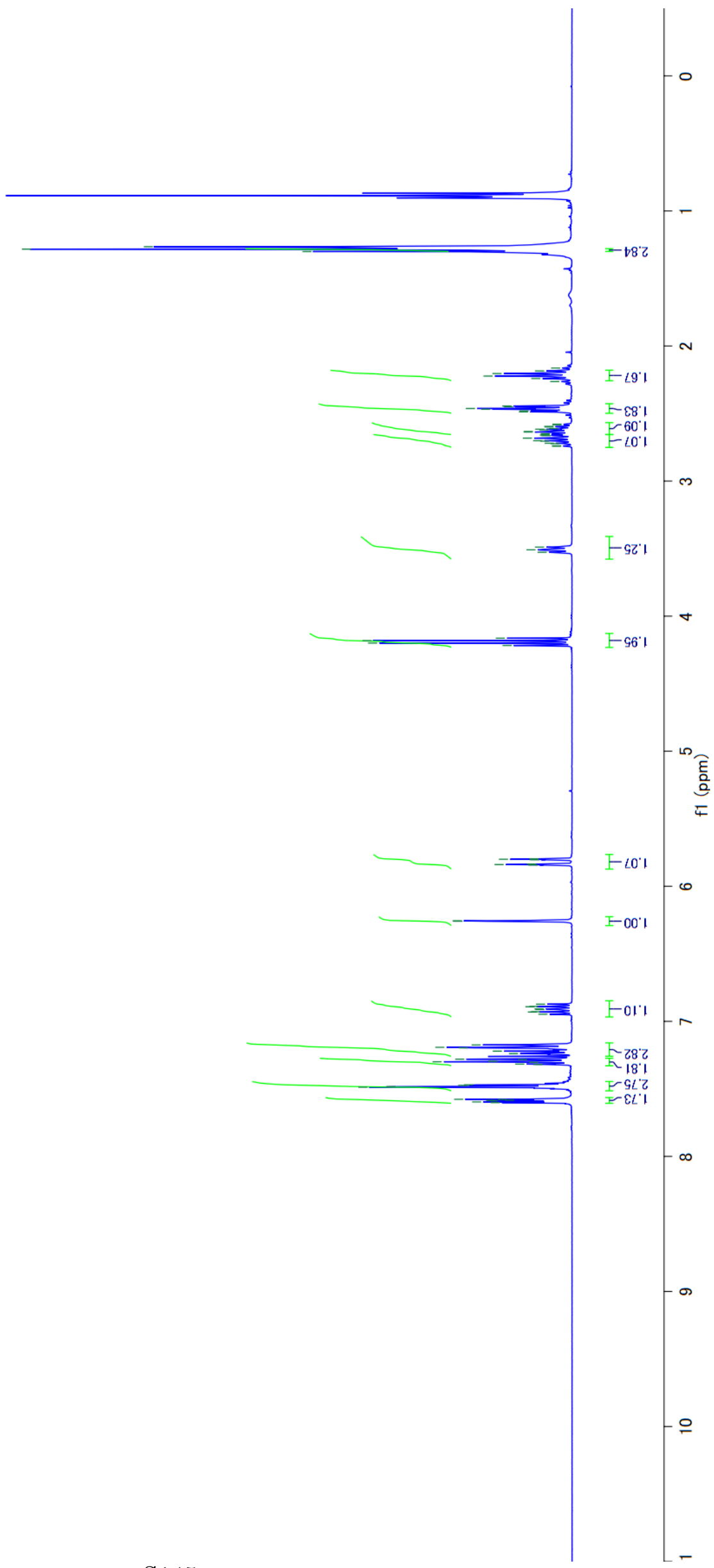


$92+1-$

$89+6 z-$

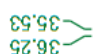

$00 \% 19-$

$87^{\circ} 09-$

$19^{\circ} 201-$

$\left.\begin{array}{l}89^{\circ} 611 \\ 99^{\circ} \mathrm{ZL}\end{array}\right]$

$\left.18^{\circ} 921\right]$

Gद'เZI]

$89.821-5$

26.821

GL'OEI

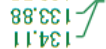

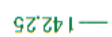

s6.9b1-

เะ'991-

$\angle 9 E L 1-$

99'६oz-

(1)

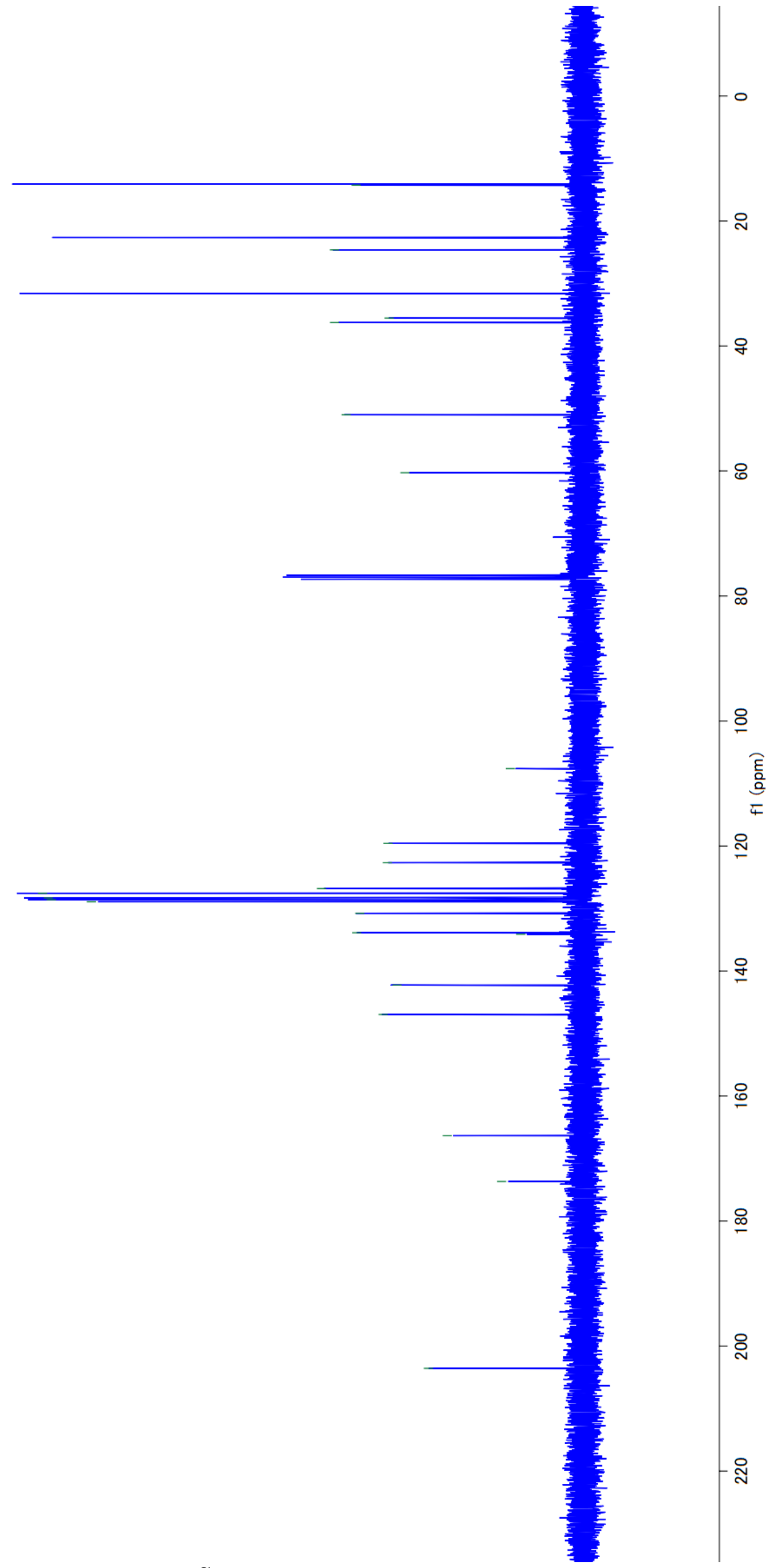

S146 


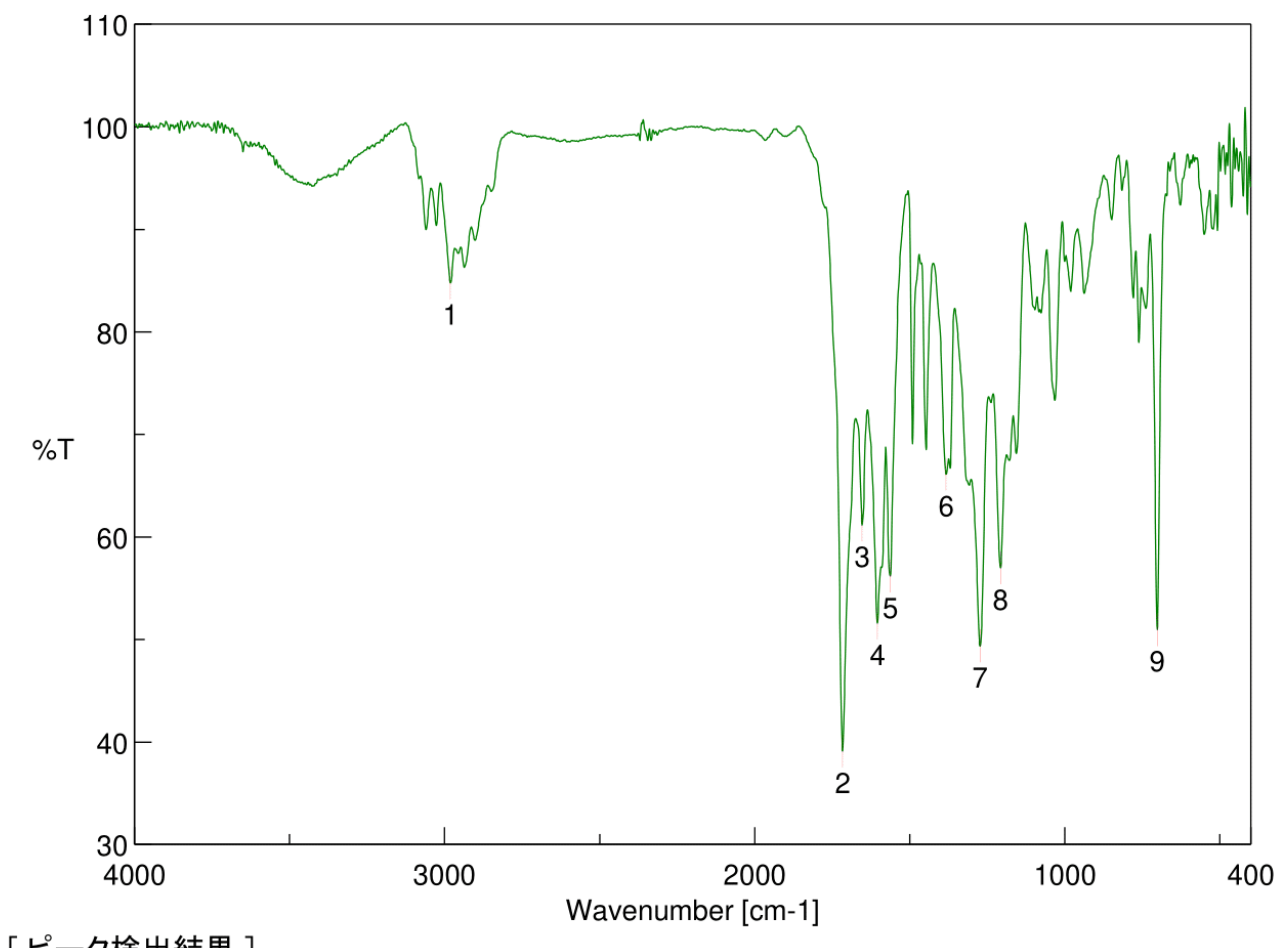

\begin{tabular}{llllll}
\multicolumn{2}{l}{ [ピーク検出結果 ] } & & & & \\
No. & 位置 & 強度 & No. & 位置 & 強度 \\
1 & 2981.41 & 84.76 & 2 & 1716.34 & 39.0942 \\
3 & 1653.66 & 61.1413 & 4 & 1604.48 & 51.5747 \\
5 & 1563.02 & 56.1717 & 6 & 1382.71 & 66.096 \\
7 & 1272.79 & 49.3289 & 8 & 1207.22 & 56.968 \\
9 & 701.962 & 50.9364 & & &
\end{tabular}

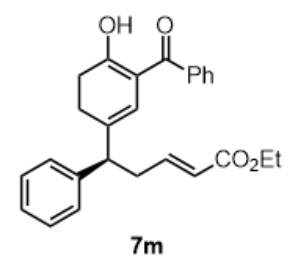




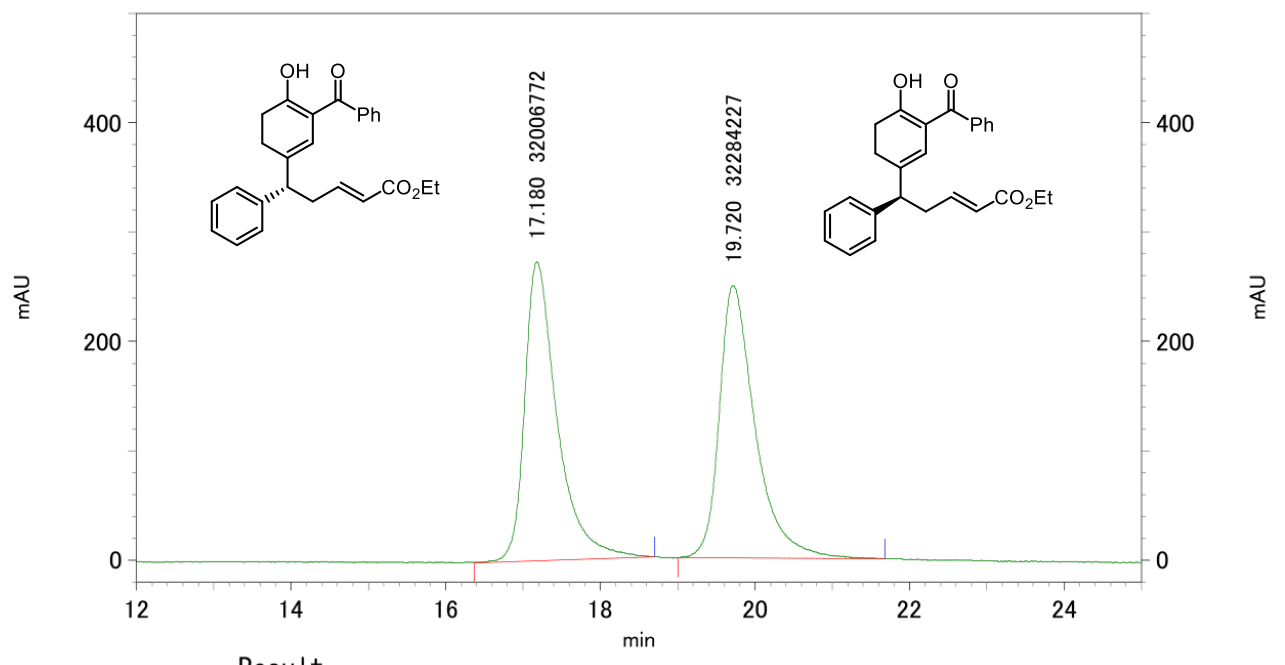

5: $210 \mathrm{~nm}, 4 \mathrm{~nm}$ 結果

Pk \# Retention time / min

Integration/\%

19. 720

50. 216

\begin{tabular}{|r|r|r|}
\hline$r-$ 外 & 100.000 \\
\hline
\end{tabular} 


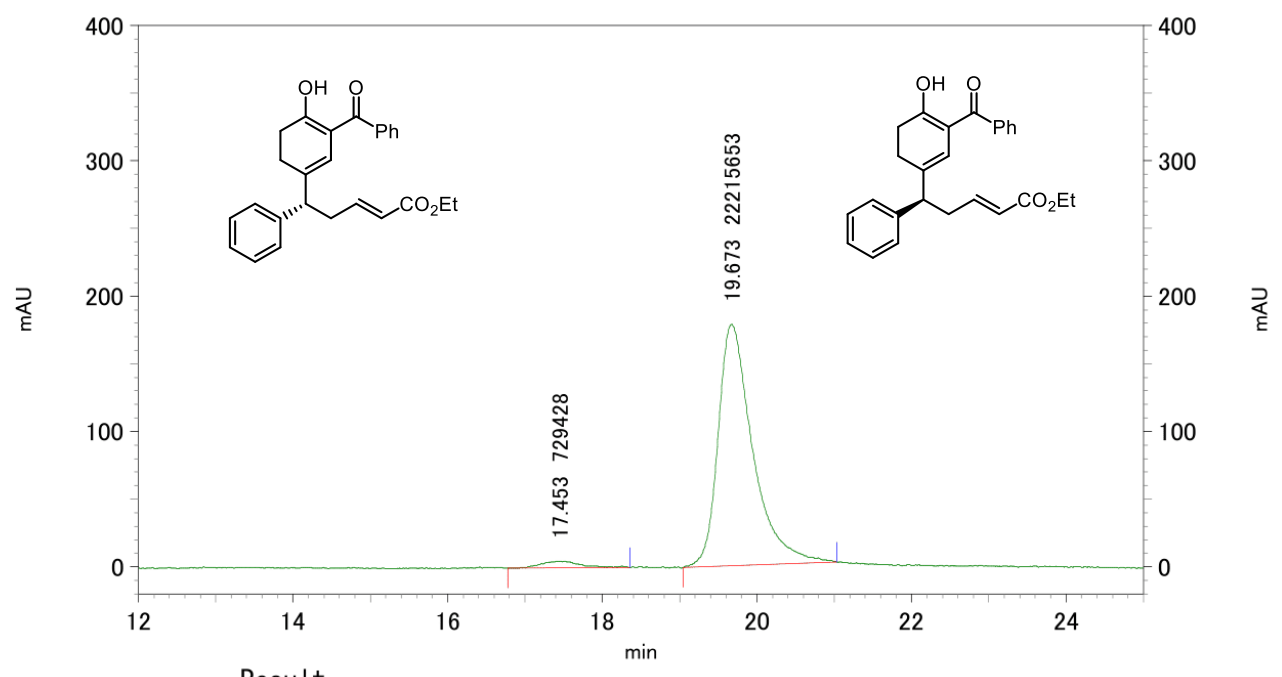

5: $210 \mathrm{~nm}, 4$ nm結果

Pk \# Retention time / min

Integration/\%

19. 673

96. 821

\begin{tabular}{|r|r|}
\hline$r-$ 外 & 100.000 \\
\hline
\end{tabular} 

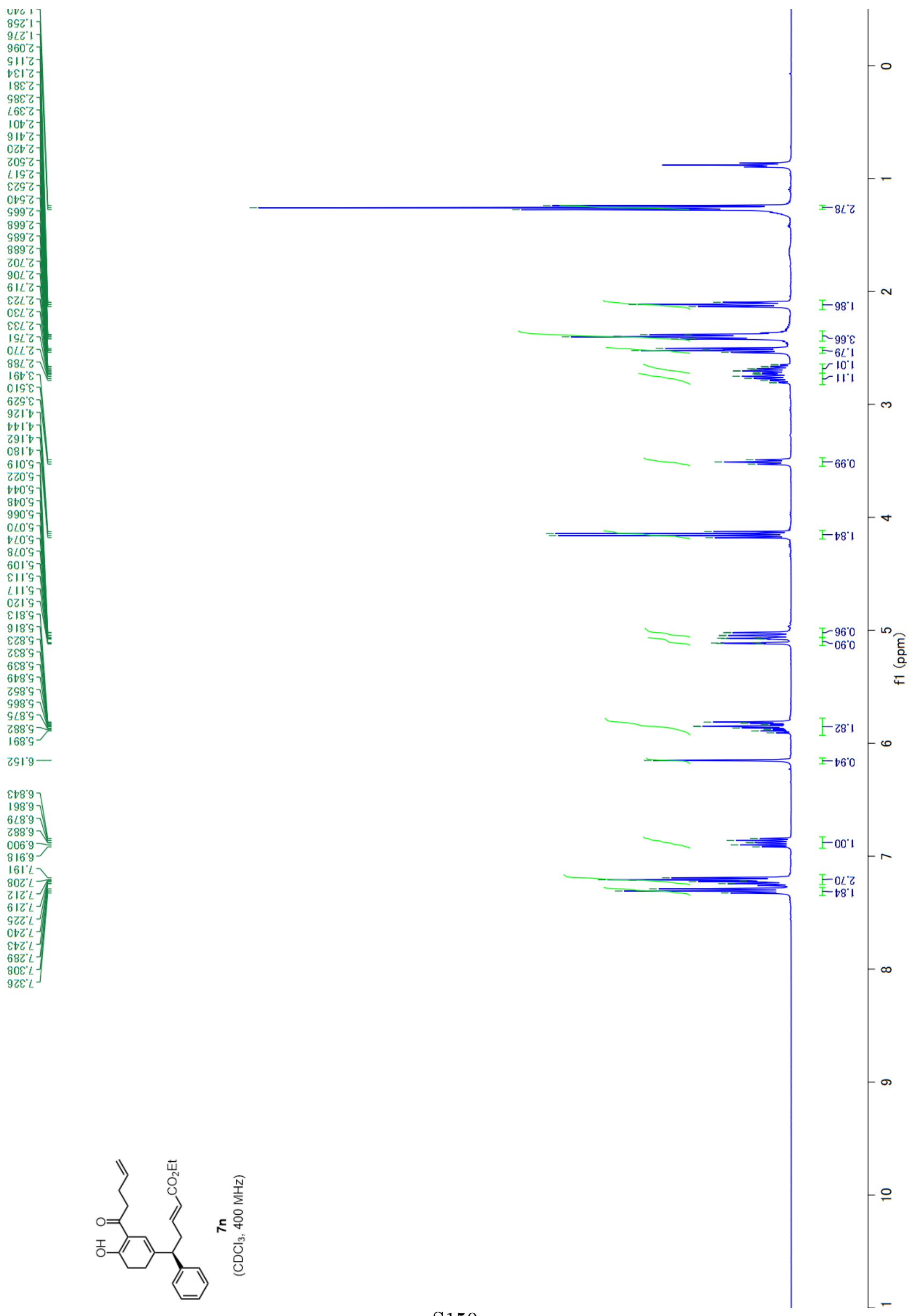



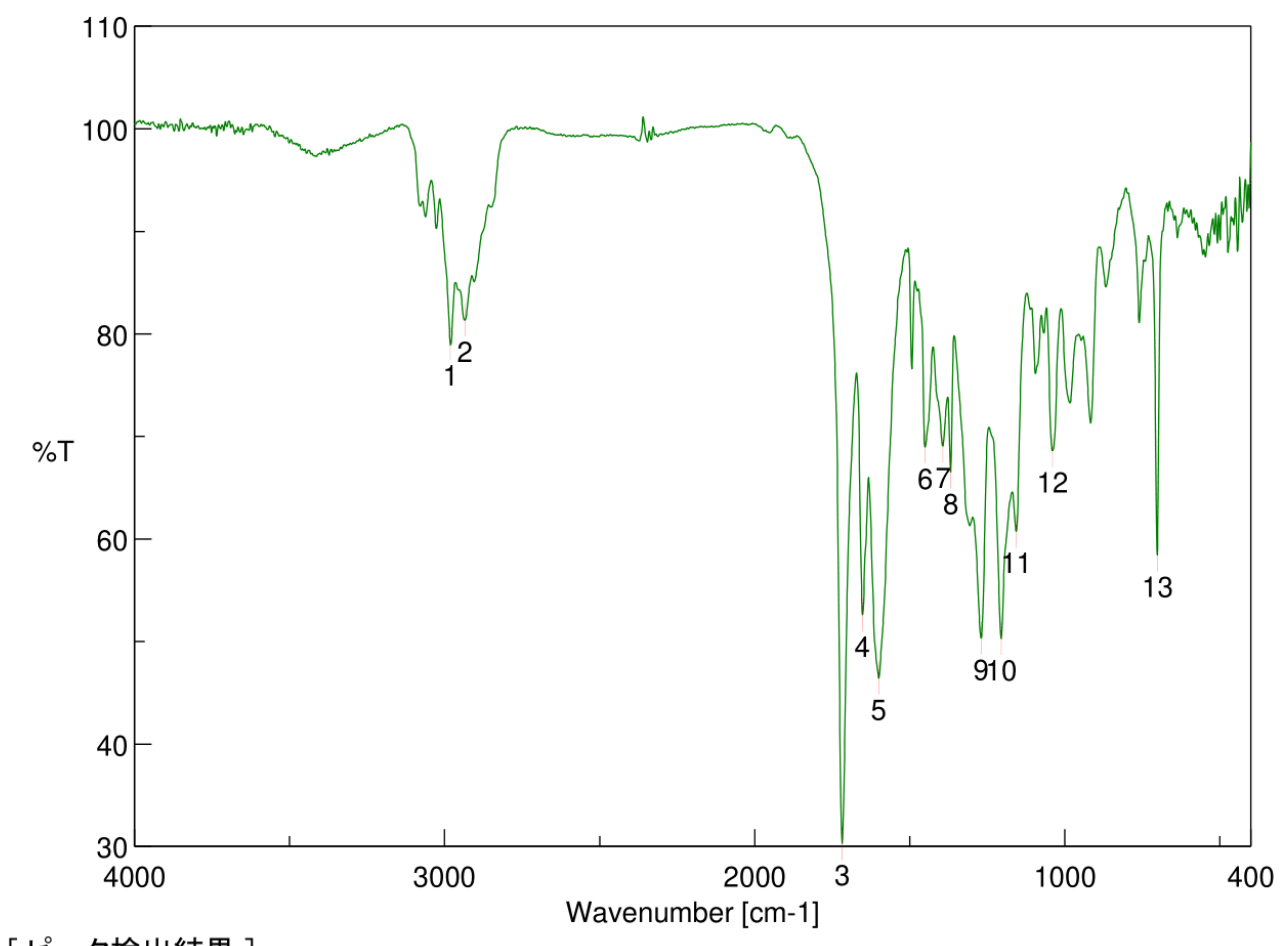

No. 位置強度

$2 \quad 2934.16 \quad 81.3444$

$\begin{array}{lll}4 & 1652.7 & 52.576\end{array}$

$\begin{array}{lll}6 & 1450.21 & 68.9578\end{array}$

$8 \quad 1368.25 \quad 66.4741$

$\begin{array}{lll}10 & 1205.29 & 50.254\end{array}$

$12 \quad 1039.44 \quad 68.5787$

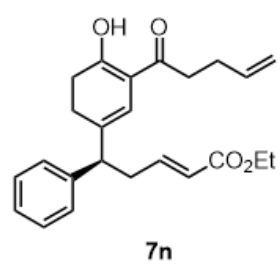




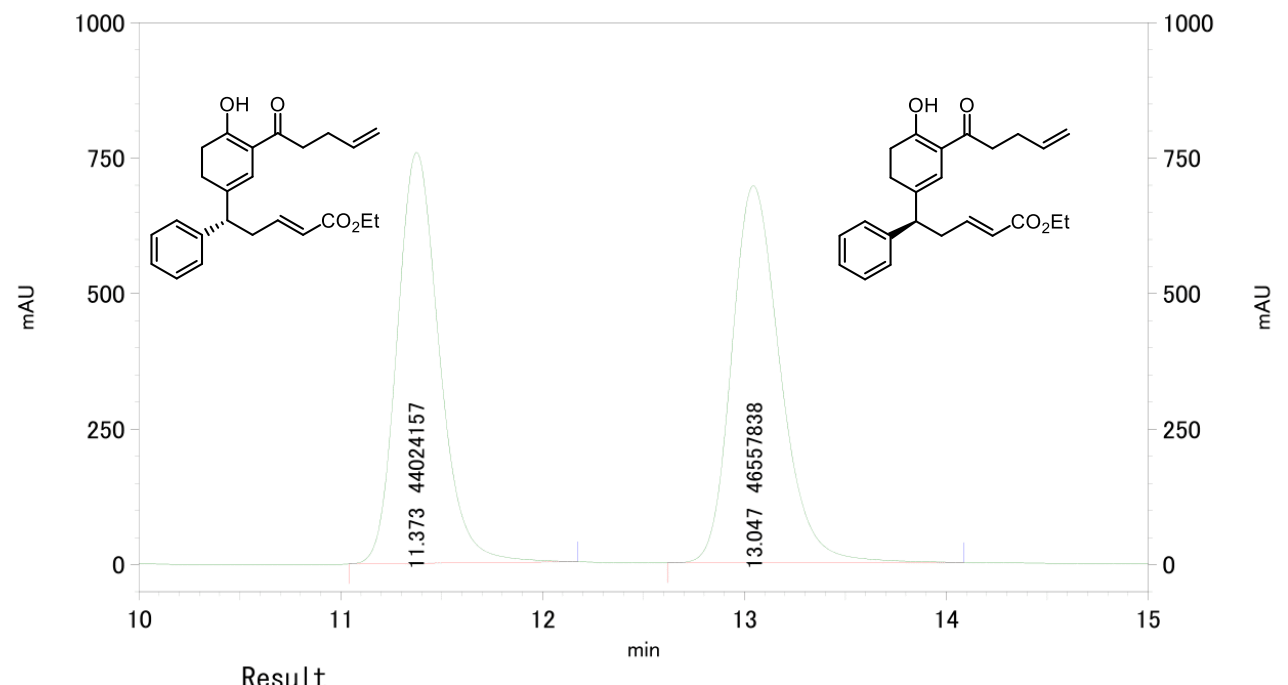

1: $253 \mathrm{~nm}, 4 \mathrm{~nm}$ 結果

Pk \# Retention time / min

Integration/ \%

11.373

51.399

\begin{tabular}{|r|r|r|}
\hline$r-$ 夕ll & 100.000 \\
\hline
\end{tabular} 

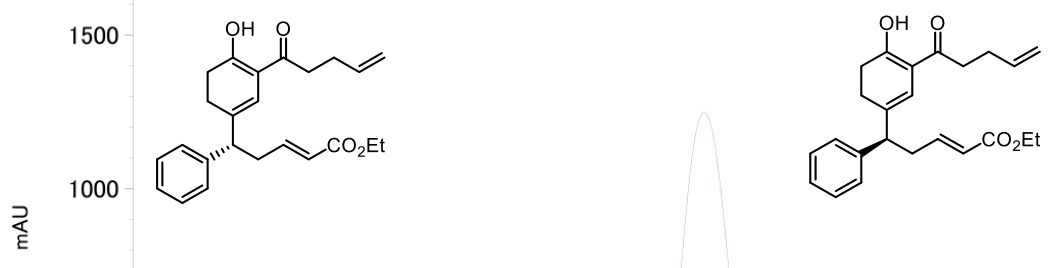

1500

500

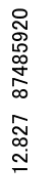

500
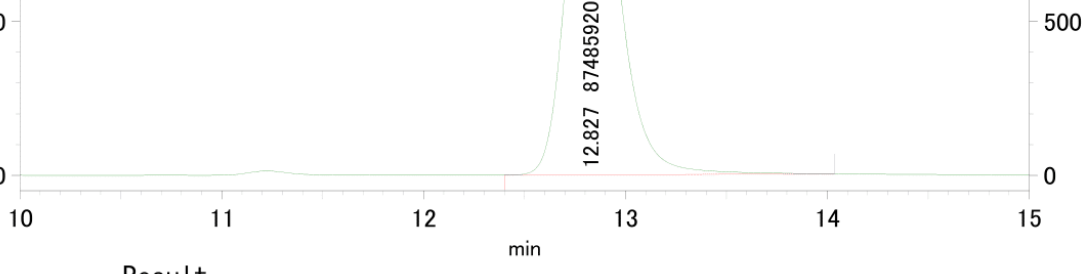

1: $253 \mathrm{~nm}, 4$ nm結果

Pk \#

Retention time / min

Integration/\%

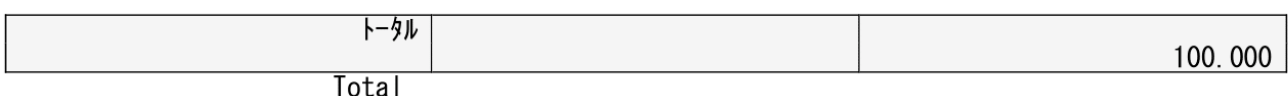




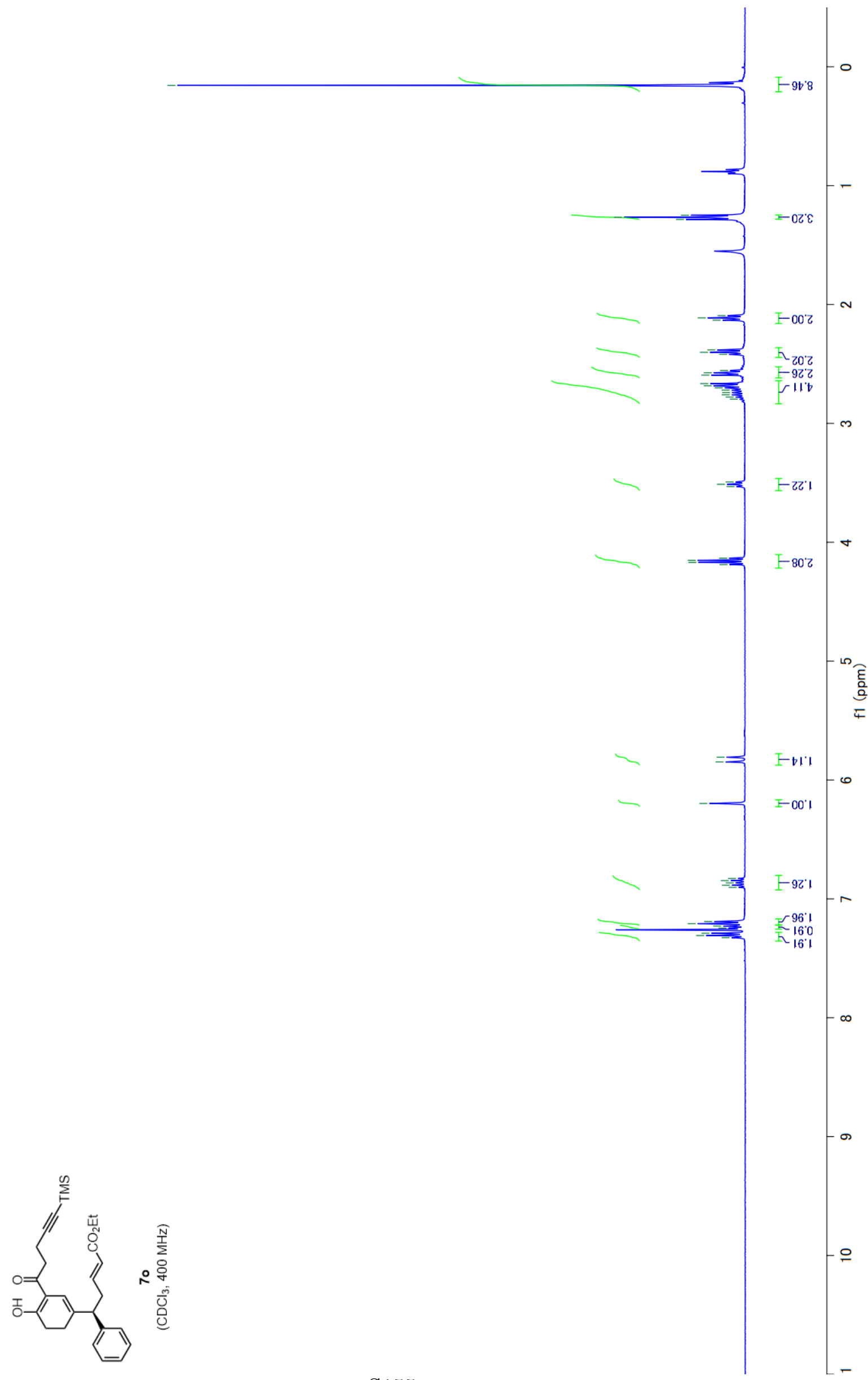




$$
t
$$




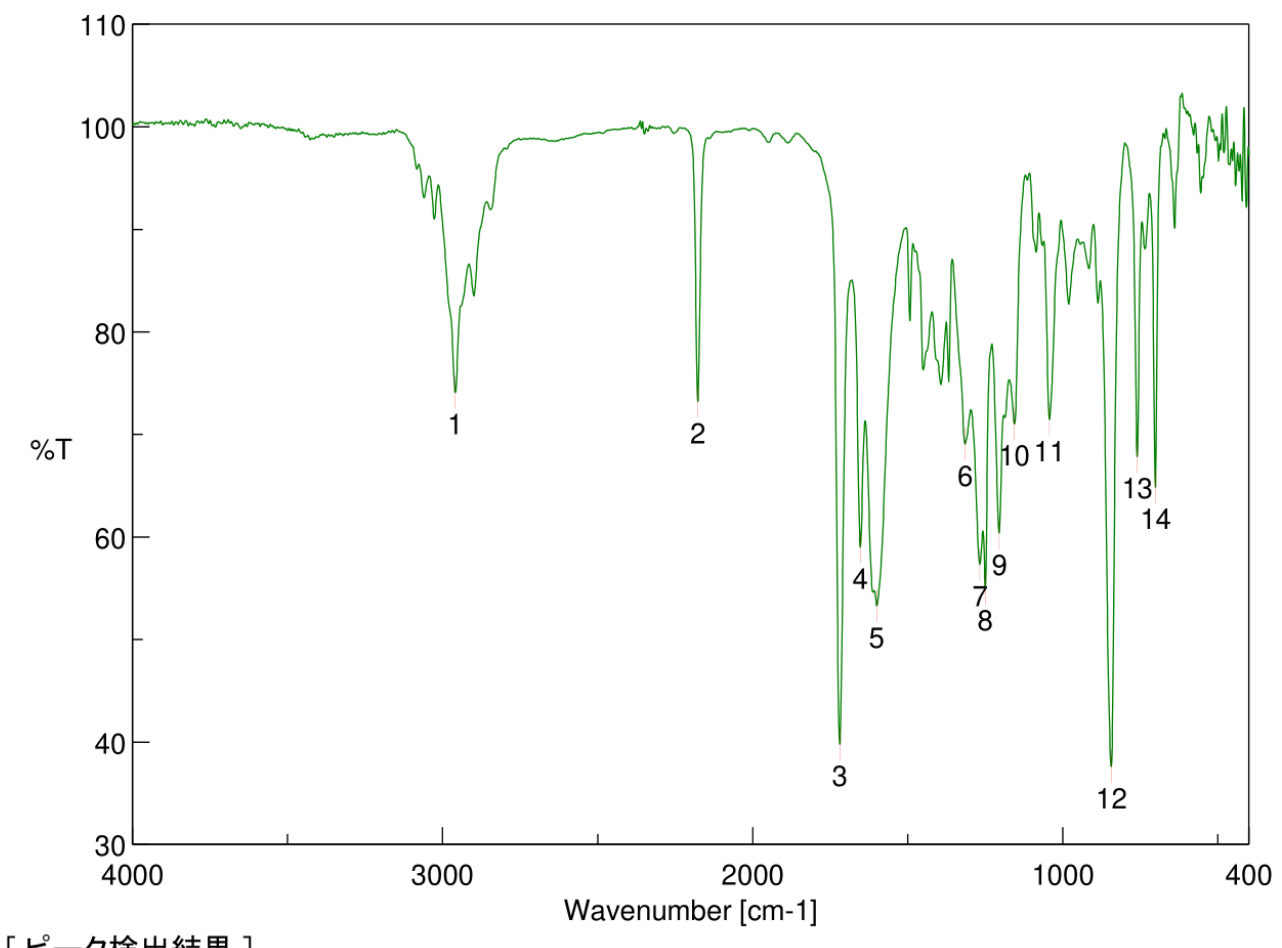

[ピーク検出結果 ]

$\begin{array}{llllll}\text { No. } & \text { 位置 } & \text { 強度 } & \text { No. } & \text { 位置 } & \text { 強度 } \\ 1 & 2959.23 & 74.0637 & 2 & 2177.24 & 73.1644 \\ 3 & 1719.23 & 39.738 & 4 & 1652.7 & 58.9963 \\ 5 & 1599.66 & 53.269 & 6 & 1315.21 & 69.0575 \\ 7 & 1267.97 & 57.2981 & 8 & 1249.65 & 54.9831 \\ 9 & 1205.29 & 60.3515 & 10 & 1156.12 & 71.009 \\ 11 & 1043.3 & 71.4487 & 12 & 843.704 & 37.5983 \\ 13 & 759.816 & 67.8089 & 14 & 700.998 & 64.8661\end{array}$




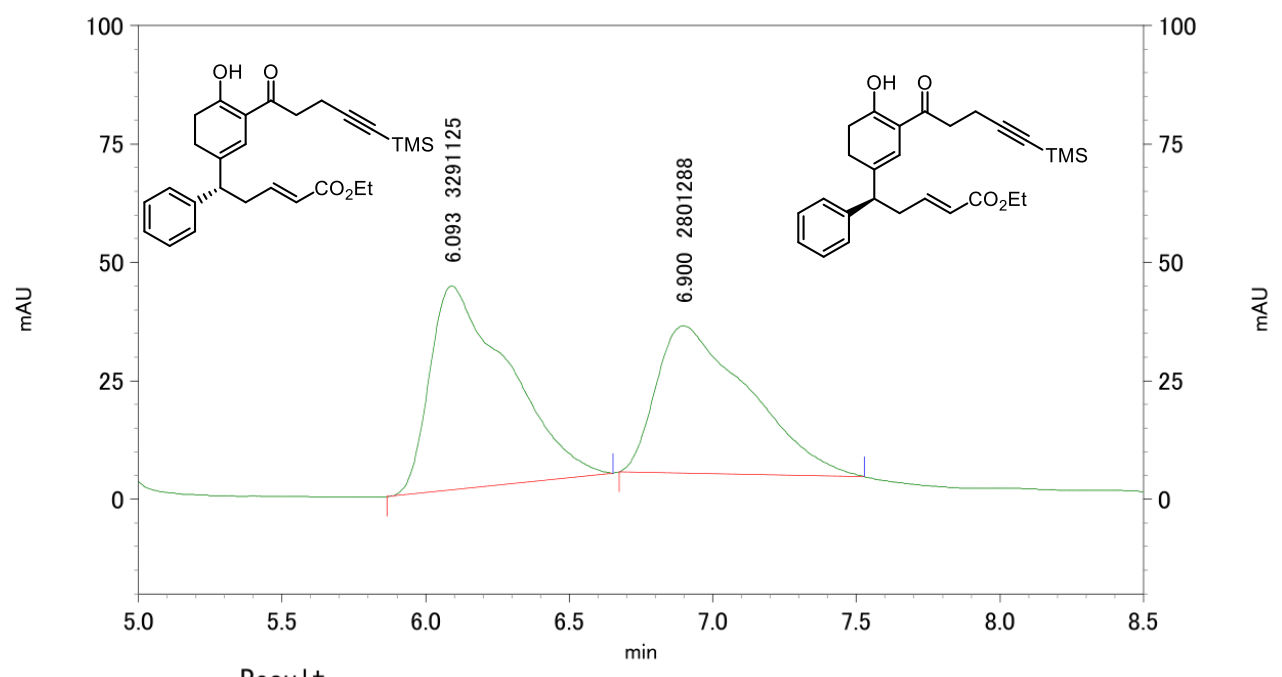

1: $319 \mathrm{~nm}, 4 \mathrm{~nm}$ 結果

Pk \# Retention time / min

Integration/\%

6.093

45. 980

\begin{tabular}{|r|r|r|}
\hline r-夕夕l & 100.000 \\
\hline
\end{tabular} 


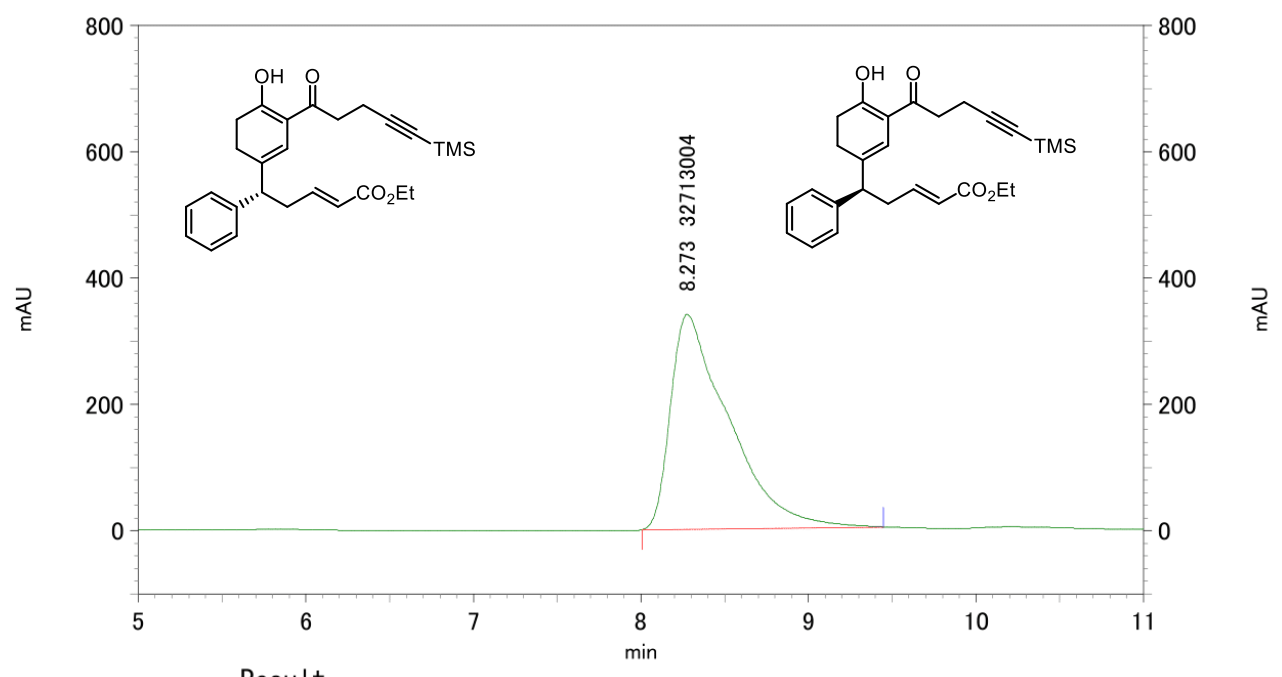

1: $319 \mathrm{~nm}, 4$ nm結果

Pk \# Retention time / min

Integration/\%

\begin{tabular}{|r|r|r|}
\hline$r-$ 舛 & 100.000 \\
\hline Total & & 1
\end{tabular} 


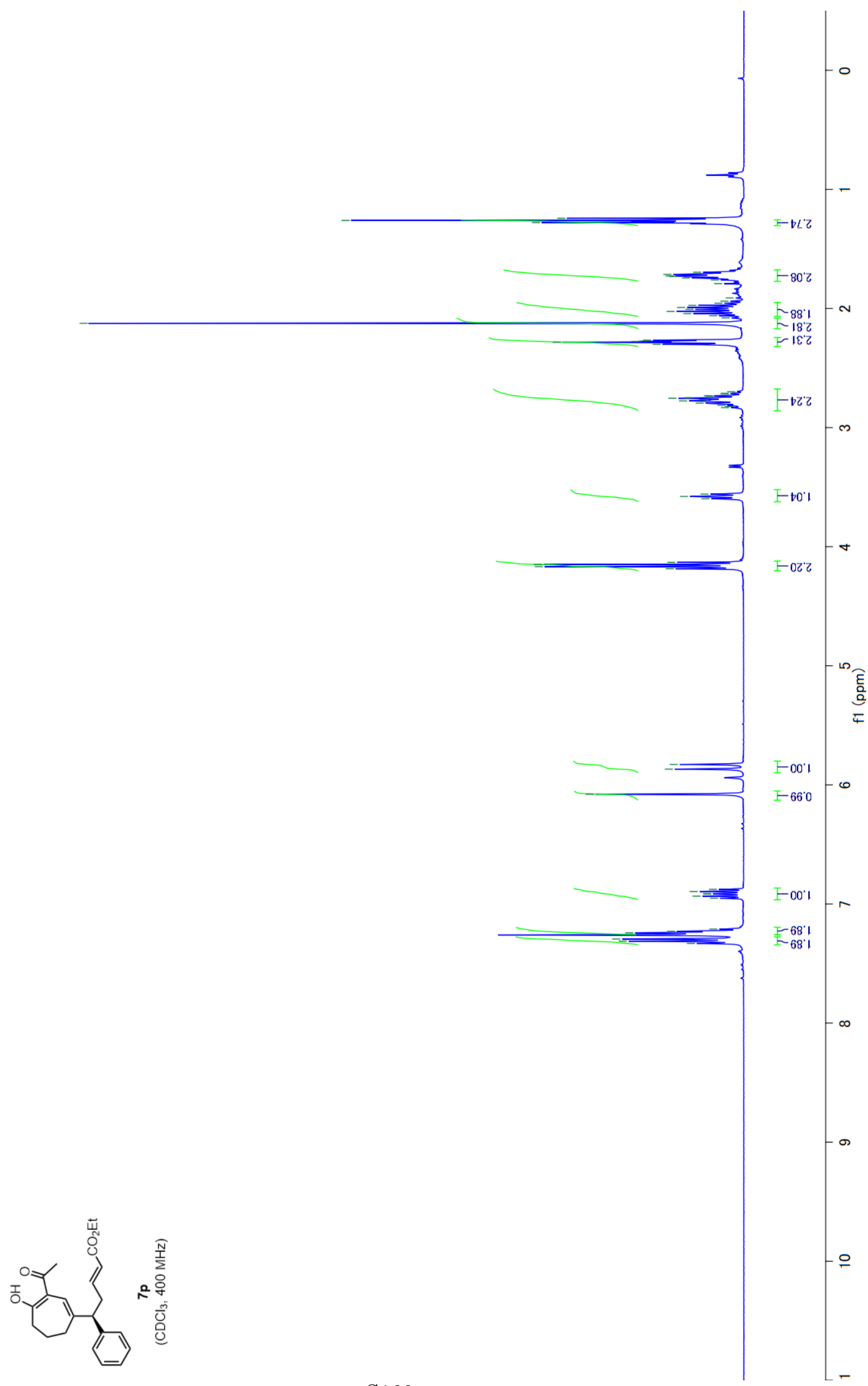




$$
t
$$




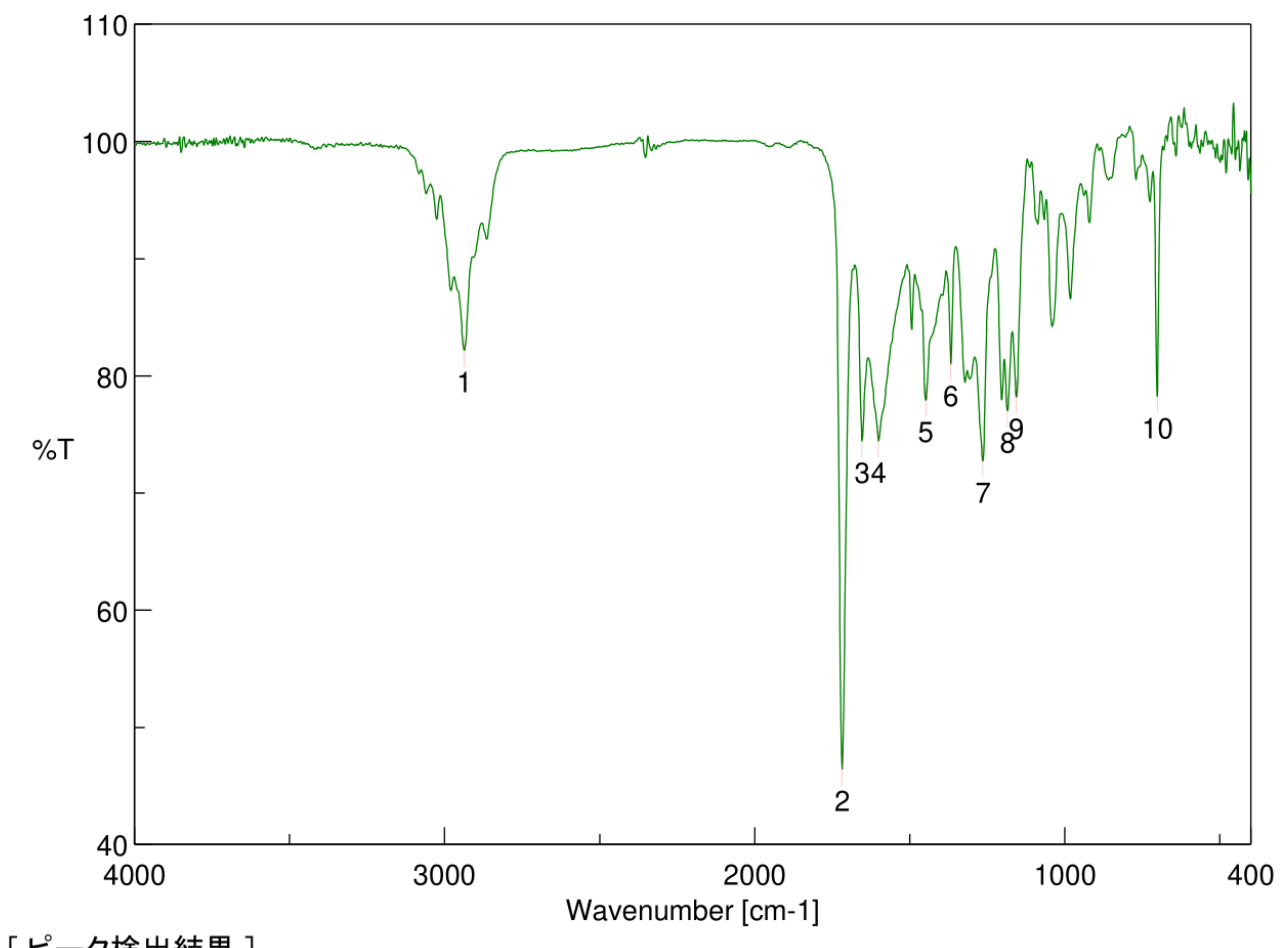

\begin{tabular}{llllll}
\multicolumn{2}{l}{ [ピーク検出結果 ] } & 強度 & No. & 位置 & 強度 \\
No. & 位置 & 29.1424 & 2 & 1718.26 & 46.4035 \\
1 & 2937.06 & 82.145 & 4 & 1600.63 & 74.4345 \\
3 & 1653.66 & 74.4461 & 6 & 1367.28 & 80.9912 \\
5 & 1448.28 & 77.8871 & 8 & 1185.04 & 77.0001 \\
7 & 1264.11 & 72.7165 & 10 & 701.962 & 78.2176
\end{tabular}

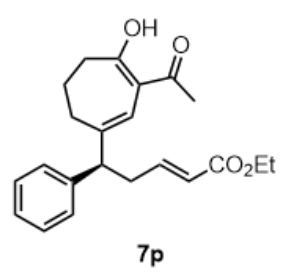




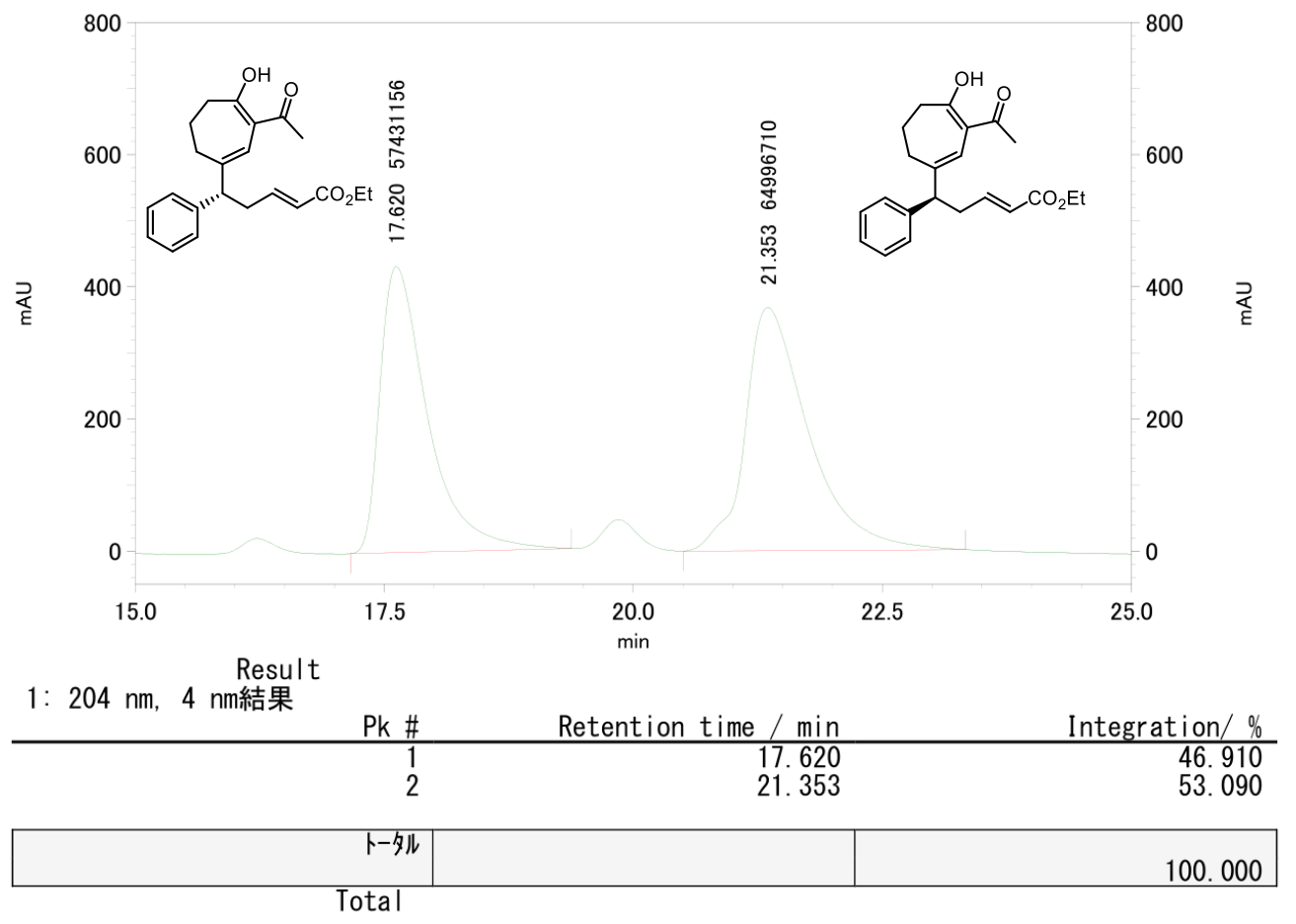




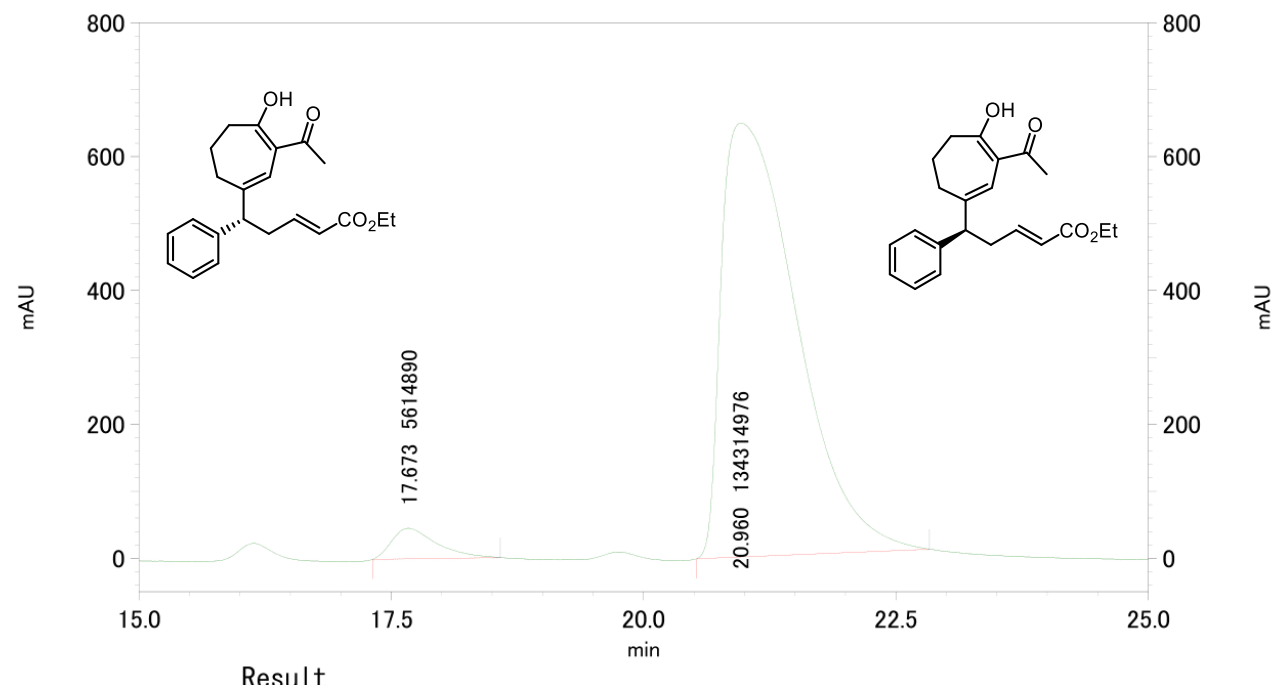

1: $204 \mathrm{~nm}, 4 \mathrm{~nm}$ 結果

$\mathrm{Pk} \# \quad$ Retention time / min

20. 960

Integration/ \%

\begin{tabular}{r|r|r|r|} 
Total &
\end{tabular}

100. 000 


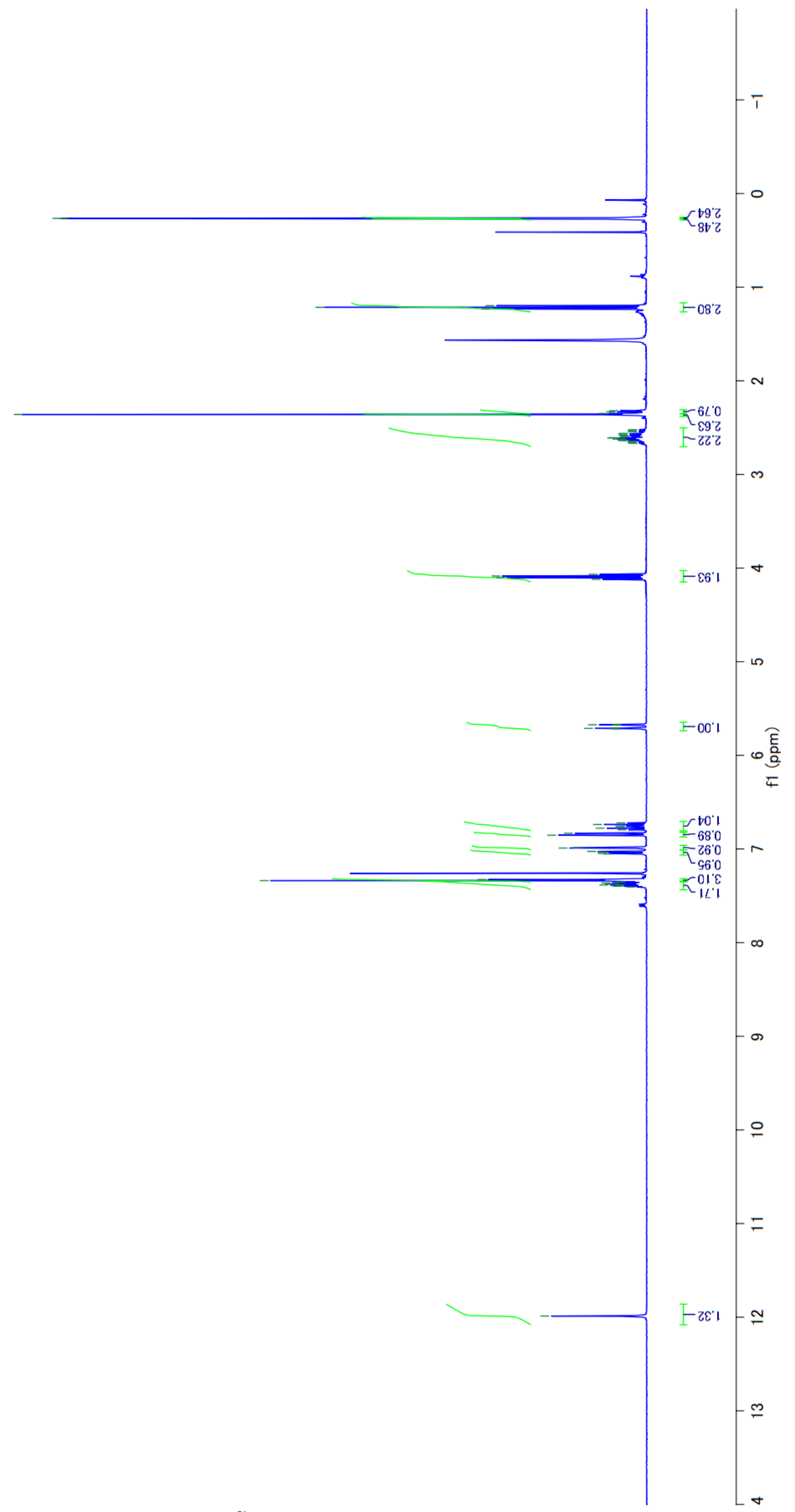


$90^{\circ} 9-$
$29^{\circ}-$

$9101-$

$16.92-$

ยเฉะ-

$0109-$

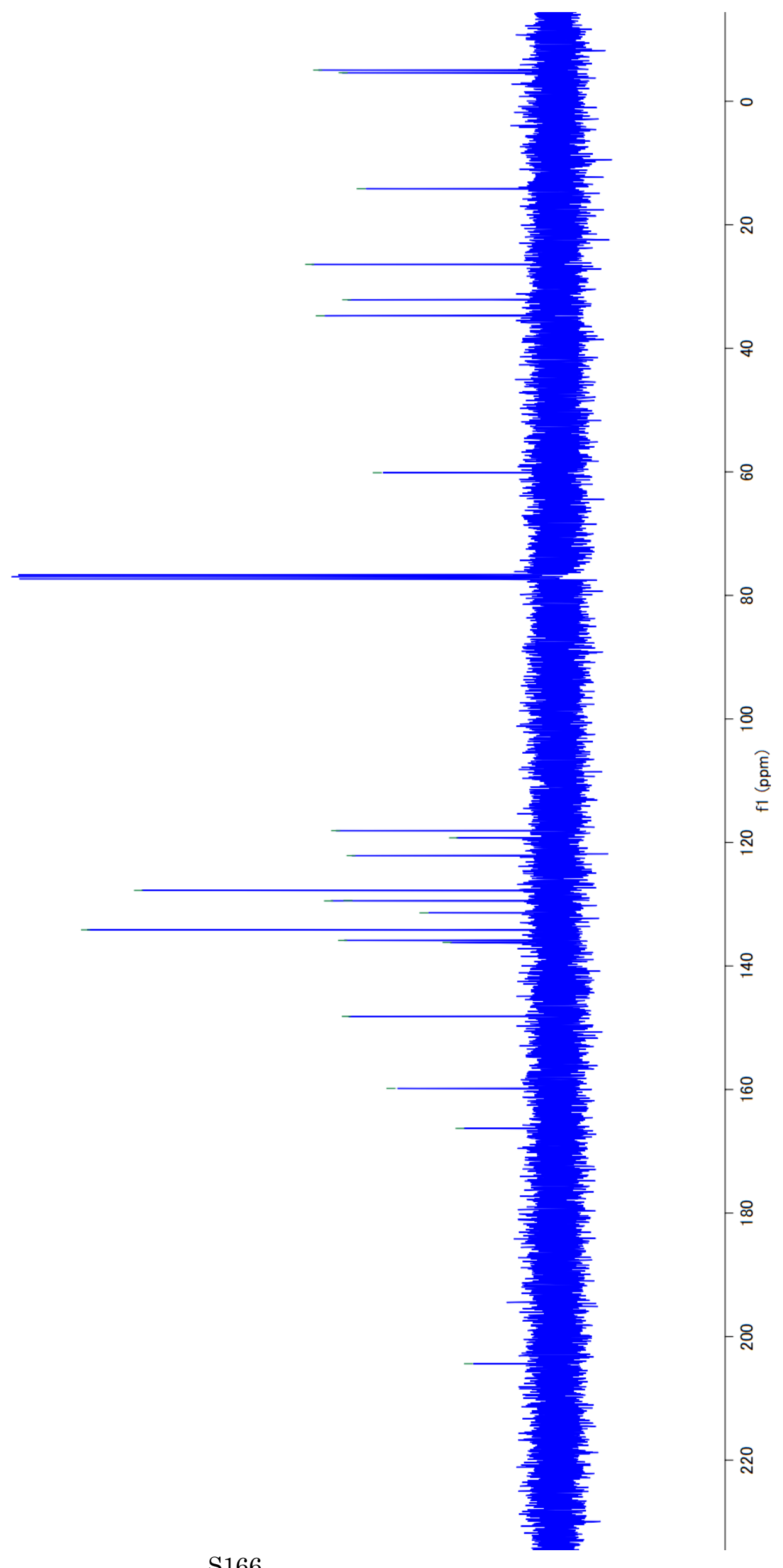

$6 \varepsilon+0 z-$

> 


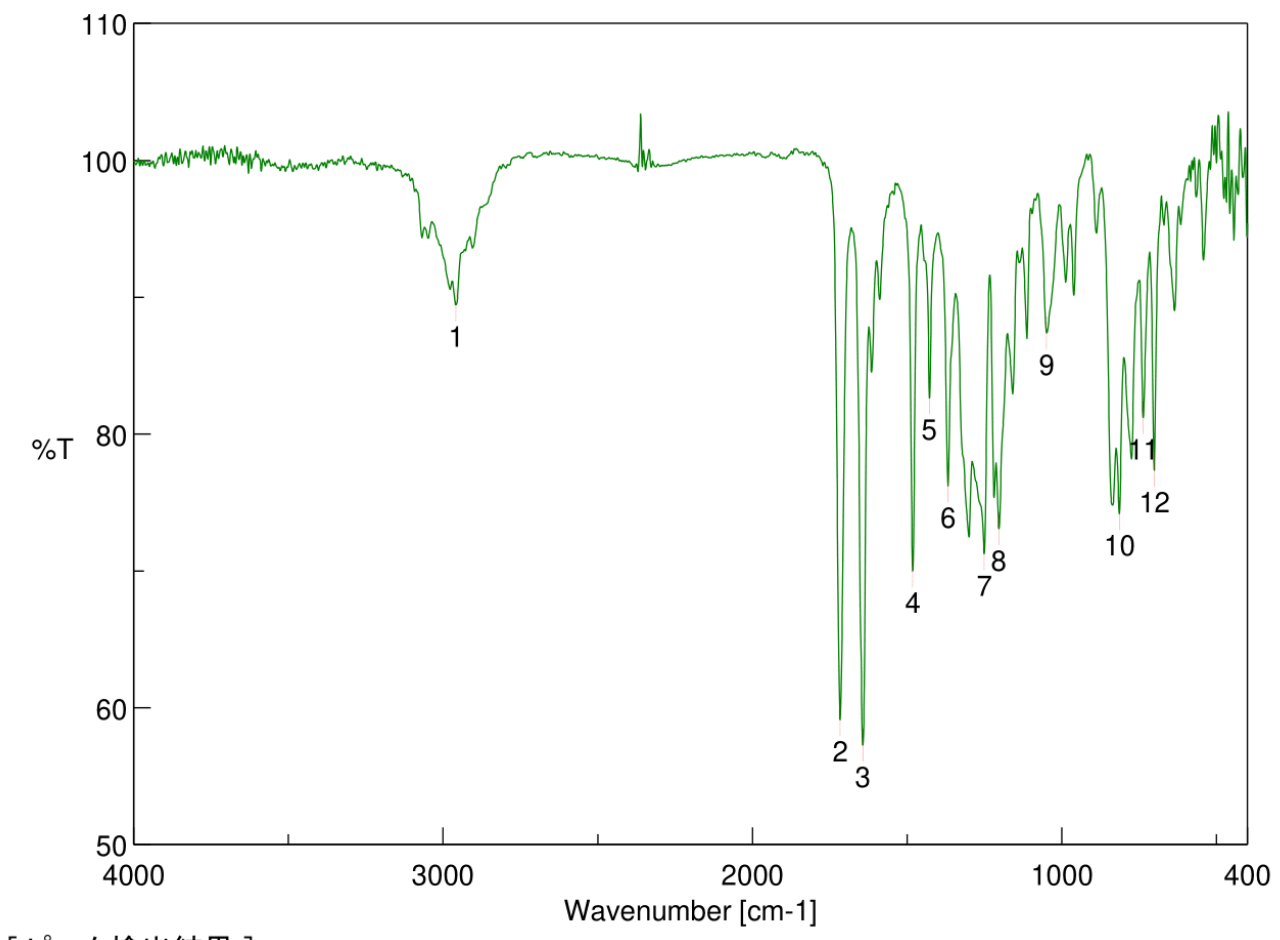

[ピーク検出結果 ]

$\begin{array}{lllllll}\text { No. } & \text { 位置 } & \text { 強度 } & \text { No. } & \text { 位置 } & \text { 強度 } & \\ 1 & 2958.27 & 89.4284 & 2 & 1716.34 & 59.1082 & \\ 3 & 1643.05 & 57.2565 & 4 & 1482.03 & 69.9693 \\ 5 & 1428.03 & 82.6429 & 6 & 1368.25 & 76.1871 \\ 7 & 1250.61 & 71.2266 & 8 & 1203.36 & 73.0695 & \\ 9 & 1049.09 & 87.366 & 10 & 813.813 & 74.1813 & \\ 11 & 736.674 & 81.1738 & 12 & 700.998 & 77.3411 & 8\end{array}$


${ }^{100^{\circ}} 0$

EtZ

$881 \%$

$9021]$

tZZ ।

ఒЪะ' -

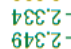

$198 ;$ ]

G09'Z

$t 0 c^{\prime} \mathrm{Z}-$

6992

099 '乙

$\angle 89^{\circ} \mathrm{Z}$

809 '

$\left.\begin{array}{l}290^{\circ} t \\ 0 \angle 0^{\circ} t\end{array}\right]$

$0 \angle 0^{\circ} \mathrm{b}$
$880^{\circ} \mathrm{b}$

901 't

111.5

$989^{\circ} \mathrm{G}=$

$689^{\circ} 9$

0149

IEL 9

$6+1.9$

$\left.99 L^{\circ} 9\right]$

$\left.\varepsilon 18^{\circ} 9\right]$

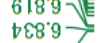

七ะ8.
0เ8.9

101.27

$\angle t \mid \cdot 2$

II $L$

GIE: $L$

$6 Z E^{\circ} L$

GEE $L$

$S+\varepsilon^{\prime} t$

$\angle t \varepsilon^{\circ} L$

$0 S E$ ' $L$ -

$\angle S E^{\circ} L-$

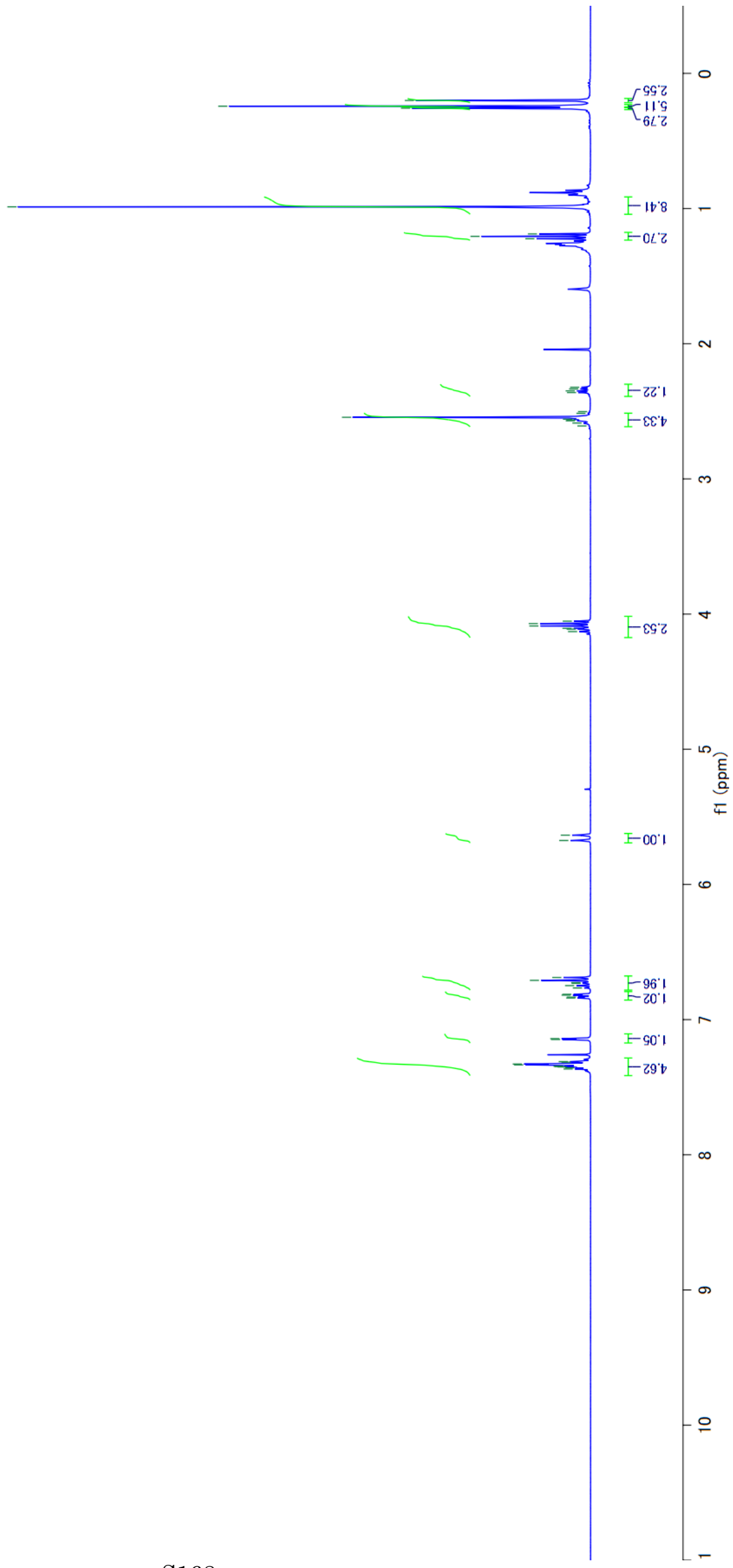


$9+1, b->$
$11+b-\gamma$

ZZto1-

$0961-$

96.92

SOTE

$98^{\circ} 9 \varepsilon$

8109

$69^{\circ} 801-$

$06.911-$

29.は1-

EะเZ1]

$\left.\angle 8^{\circ} \angle 21\right]$

ว9เ

10 हE।

06.

$06801-$

tt $991-$

b乙LLI-

$6 \varepsilon^{\prime} 661-$<smiles>CCCCC(CC=C[Se])c1ccc(OCC)c(C(C)=O)c1</smiles>

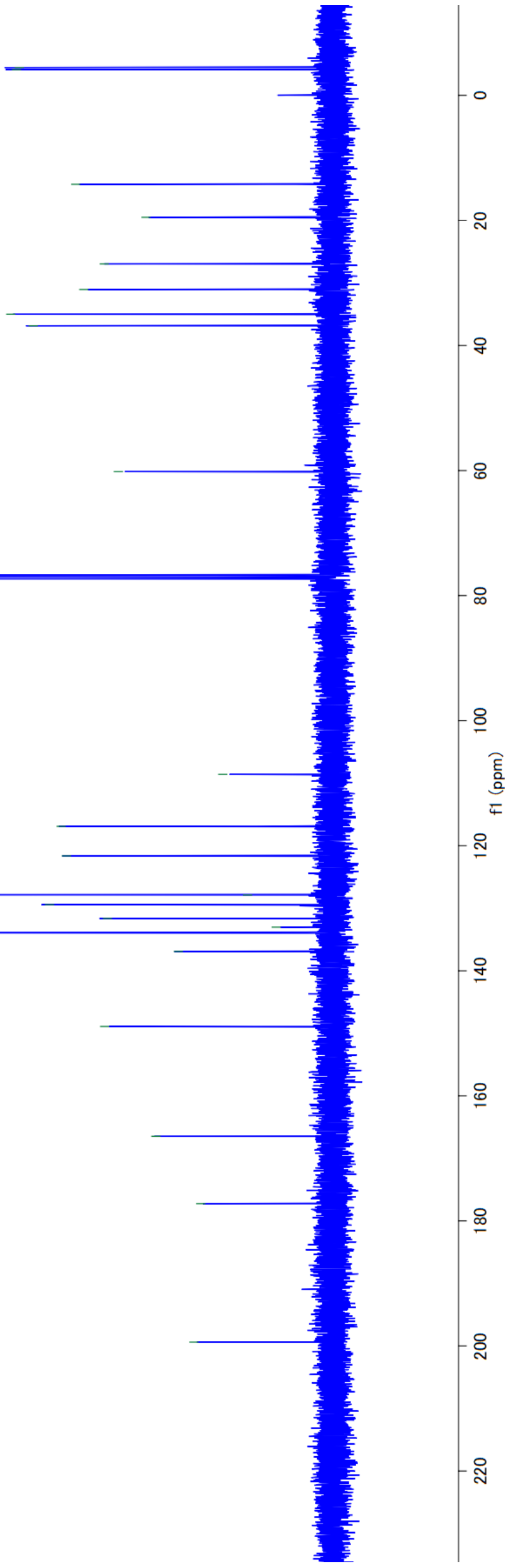




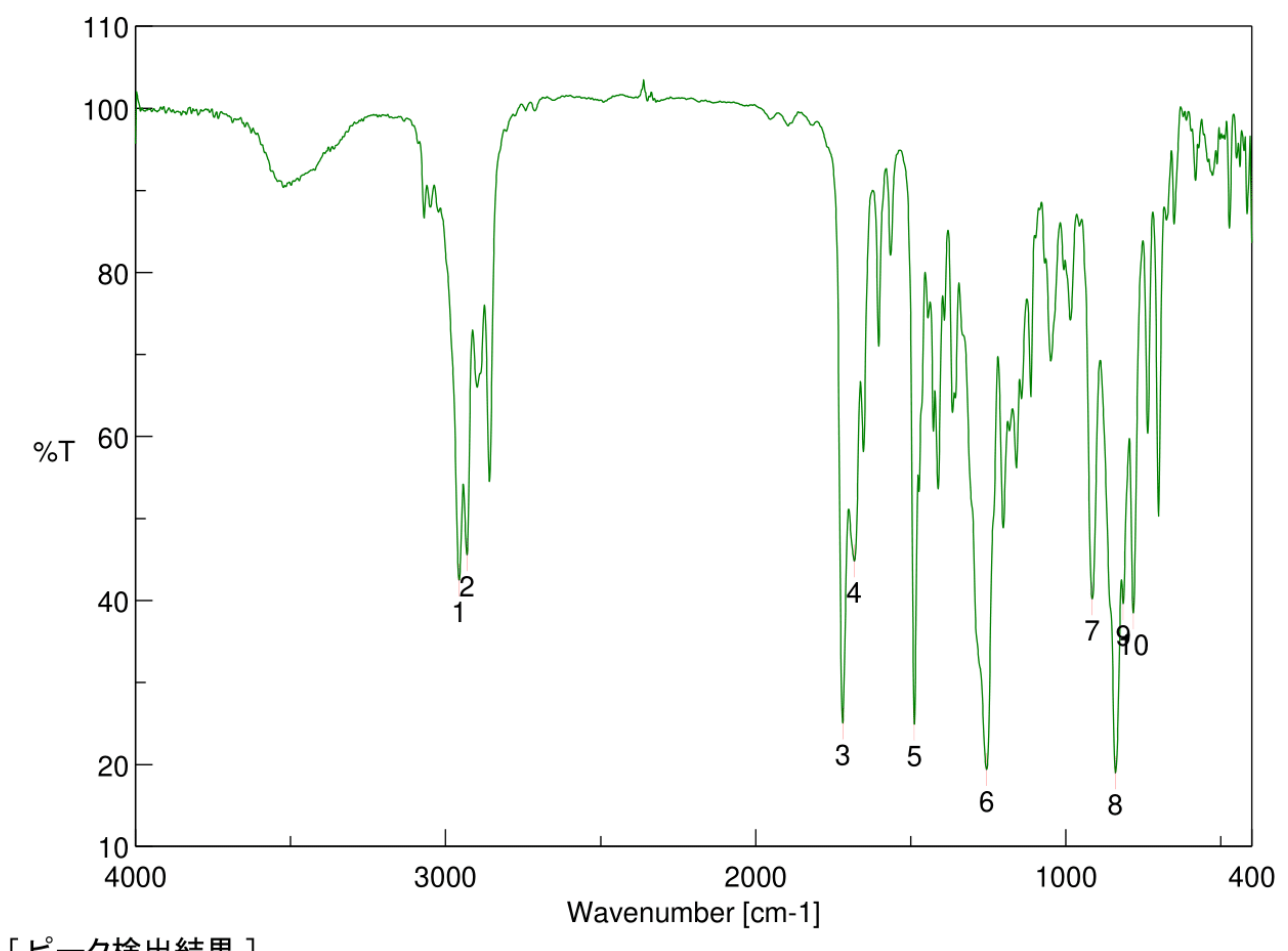

\begin{tabular}{llllll}
\multicolumn{2}{l}{ [ ピーク検出結果 ] } & 強度 & No. & 位置 & 強度 \\
No. & 位置 & 29. & 2 & 2931.27 & 45.5208 \\
1 & 2956.34 & 42.4497 & 4 & 1682.59 & 44.807 \\
3 & 1719.23 & 25.0148 & 6 & 1255.43 & 19.3812 \\
5 & 1488.78 & 24.8913 & 8 & 839.847 & 18.9391 \\
7 & 915.058 & 40.1795 & 10 & 781.993 & 38.4543
\end{tabular}

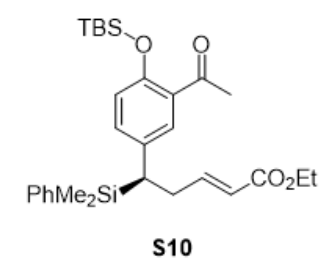


291.t

88157

902 t

$008^{\circ} \mathrm{b}$

El8't

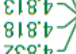

${ }_{986^{\circ} 9} \mathrm{9}=$

$\angle 06^{\circ} 9$

$\left.9 \mathrm{Z6} 6^{\circ}\right]$

St6.9

$1966^{\circ}$

$786^{\circ}{ }^{\circ}$

$9 \varepsilon+\lfloor$

$2 t+i)$

89t

Egt

$[1, L]$

$88 Z$ -

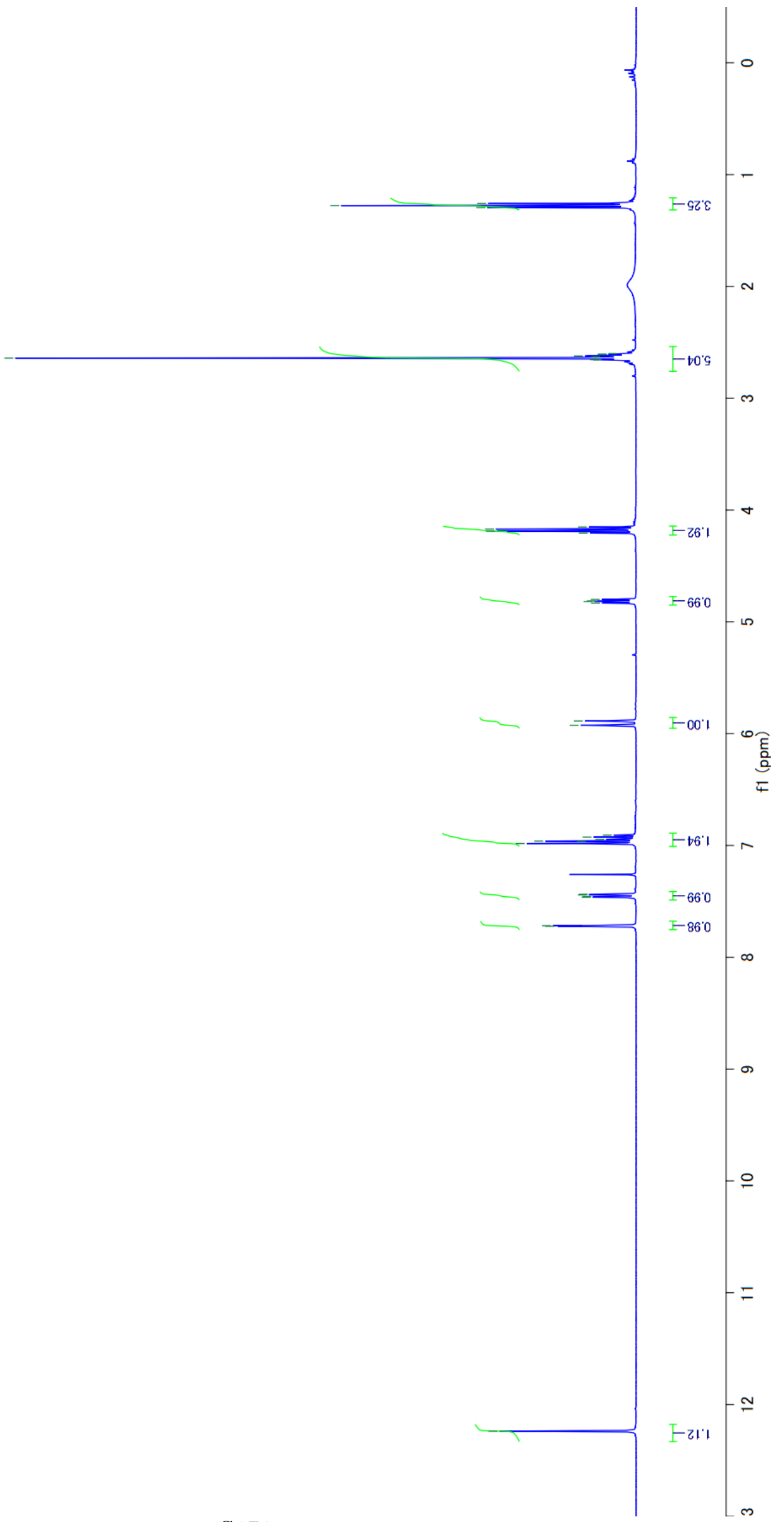


zz॰t

$0 L^{\circ} 9 z-$

s.1t -

$1009-$

$6 Z Z L$

$E L 811$
$\angle E 611$

$9 b^{\circ}$ b2 -

L8. $21-$

$68^{\circ} \varepsilon 1>$
$\left.96^{\circ}\right)$

ต6ะ $1-$

$10^{\circ} 291-$

LI.991-

ttotoz-

$=\sum_{\substack{0 \\ 0}}^{\frac{\pi}{0}}$

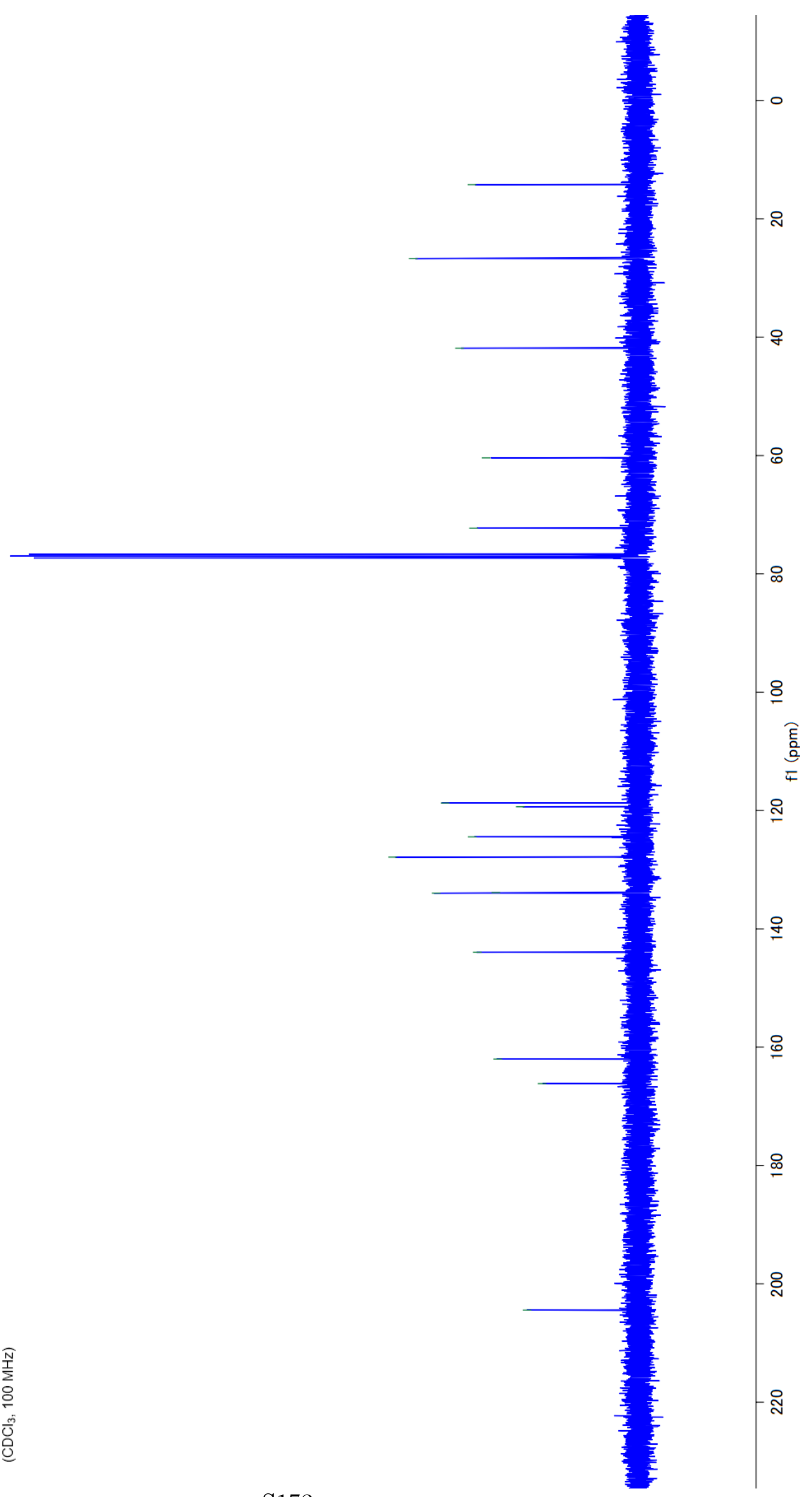

S172 


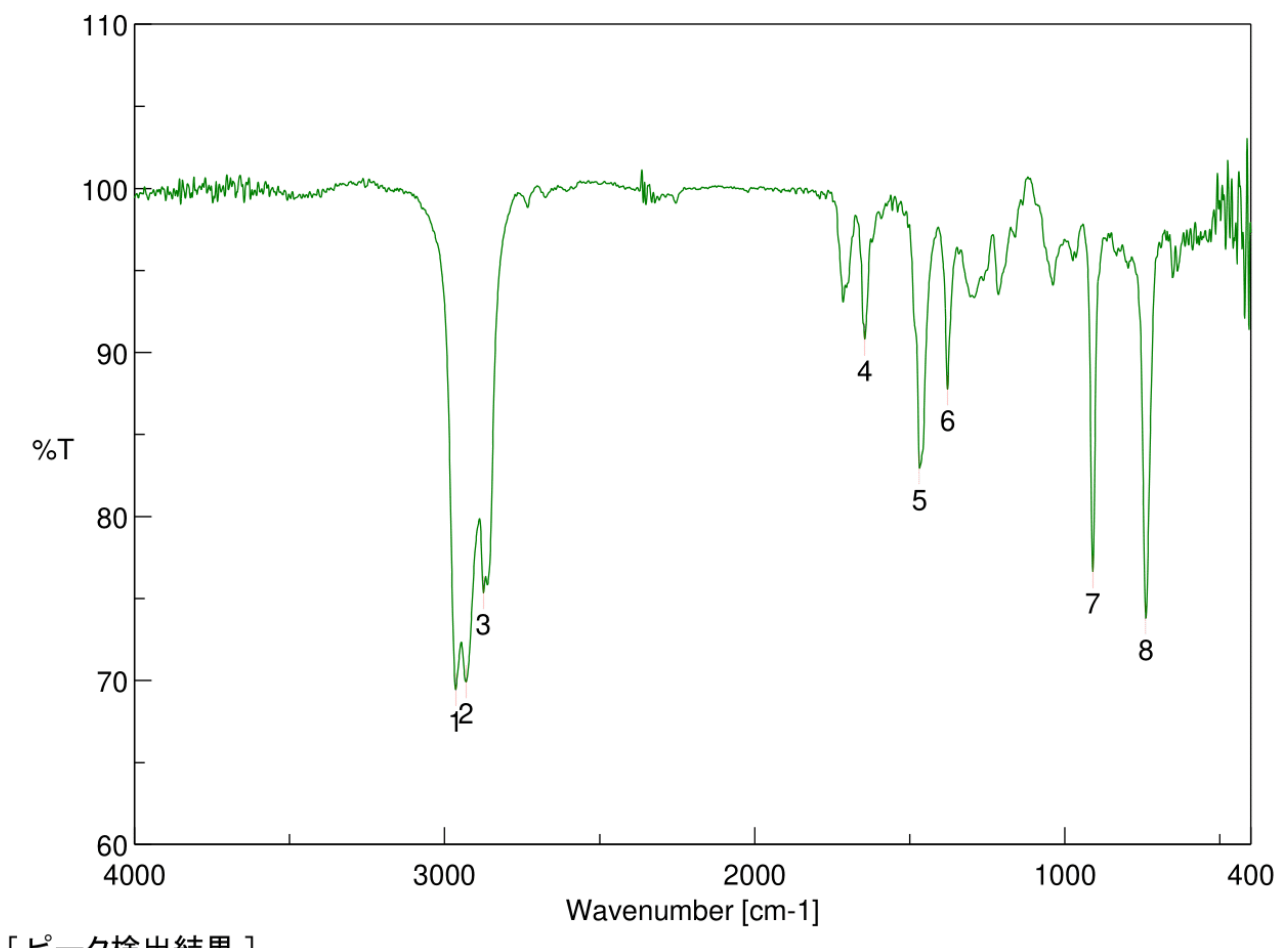

\begin{tabular}{|c|c|c|c|c|c|}
\hline \multicolumn{6}{|c|}{ [ ピーク検出結果 ] } \\
\hline No. & 位置 & 強度 & No. & 位置 & 強度 \\
\hline 1 & 2964.05 & 69.4299 & 2 & 2931.27 & 69.9094 \\
\hline 3 & 2874.38 & 75.3289 & 4 & 1644.98 & 90.7958 \\
\hline & 1468.53 & 82.9338 & 6 & 1377.89 & 87.7478 \\
\hline 7 & 910.236 & 76.6193 & 8 & 738.603 & 73.78 \\
\hline
\end{tabular}

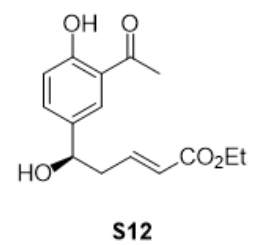


$297^{\circ} 1>$

088'

$S 19^{\circ} Z$
$Z Z 9^{\circ} \mathrm{Z}$

$969.7]$

$169^{\circ} z-$

$059^{\circ} \mathrm{Z}$

099 '

$176^{\circ} \mathrm{\circ}-$

291't

$881^{\circ}$ '

$90 z^{\circ} \mathrm{J}$

$\varepsilon 08$ t

418.6

ZZ8.

$188^{\circ} 9$

$0766^{\circ}$

$268^{\circ} 9$

$\left.\begin{array}{l}116^{\circ} 9 \\ 186^{\circ}\end{array}\right]$

096.9 -

$796^{\circ} 9$

$8966^{\circ}$

L

E8t $L$

$860^{\circ} \mathrm{L}$

bogt

EOL $L T$

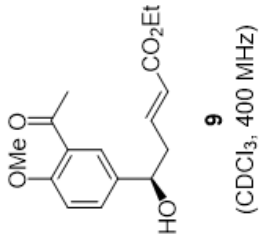

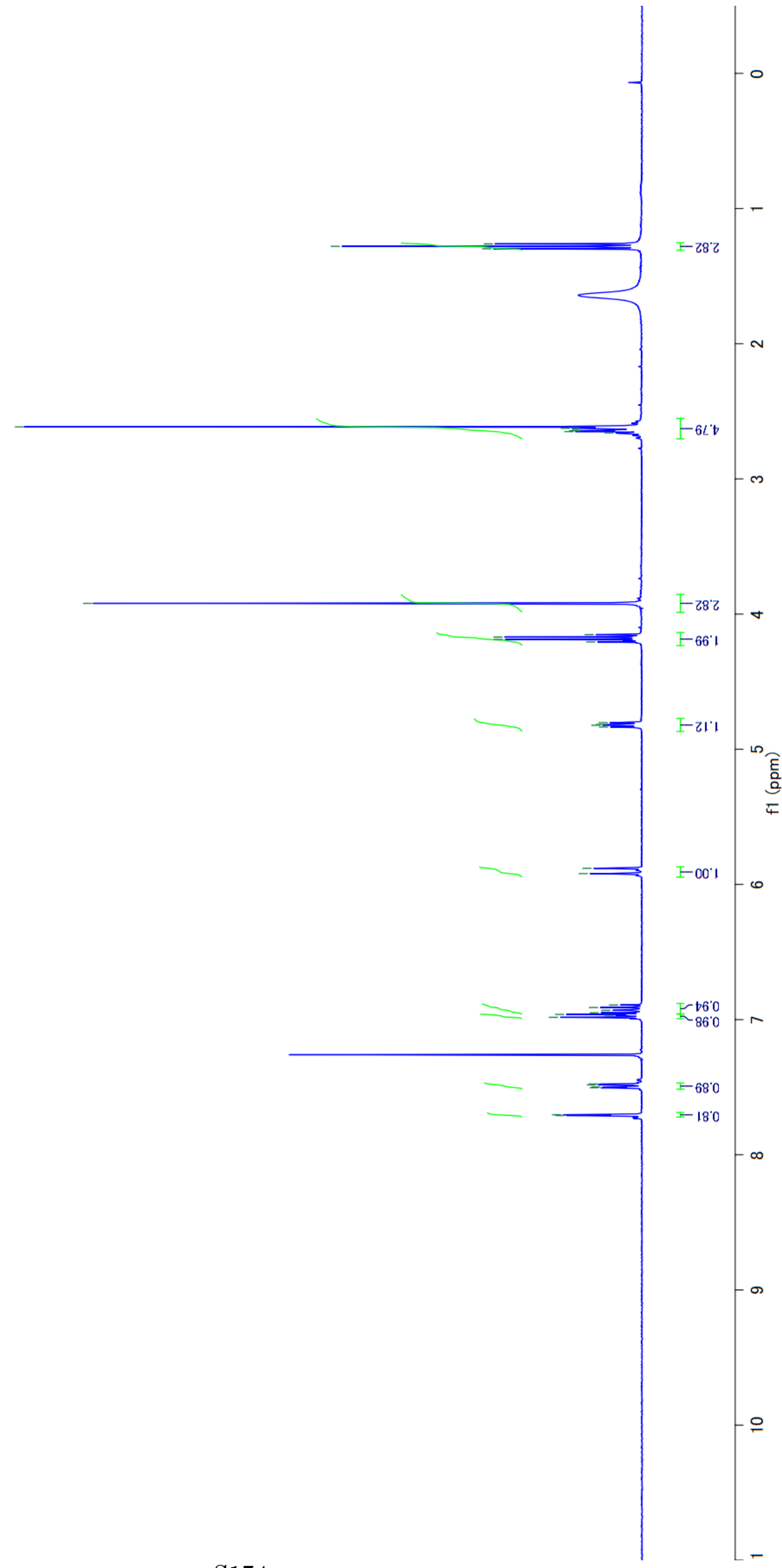


$22+51-$

๕ะเะ-

$89.16-$

५9ง૬-

๕ะ09-

$L Z Z L$

$88111-$

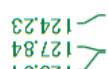

t0.821

99.

$0 \varepsilon+t)$

$89.891-$

๕ช'991-

1 I $661-$

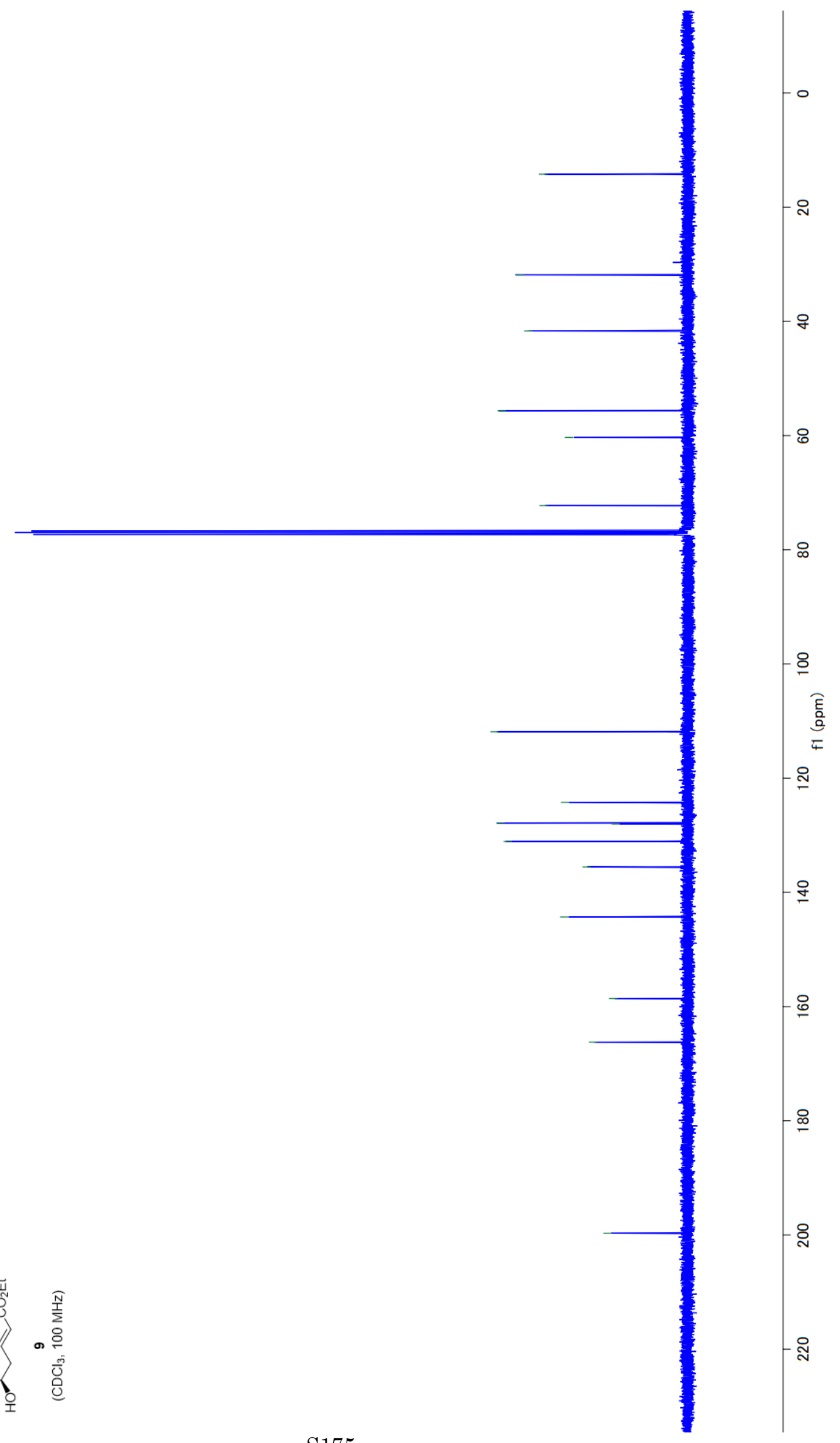




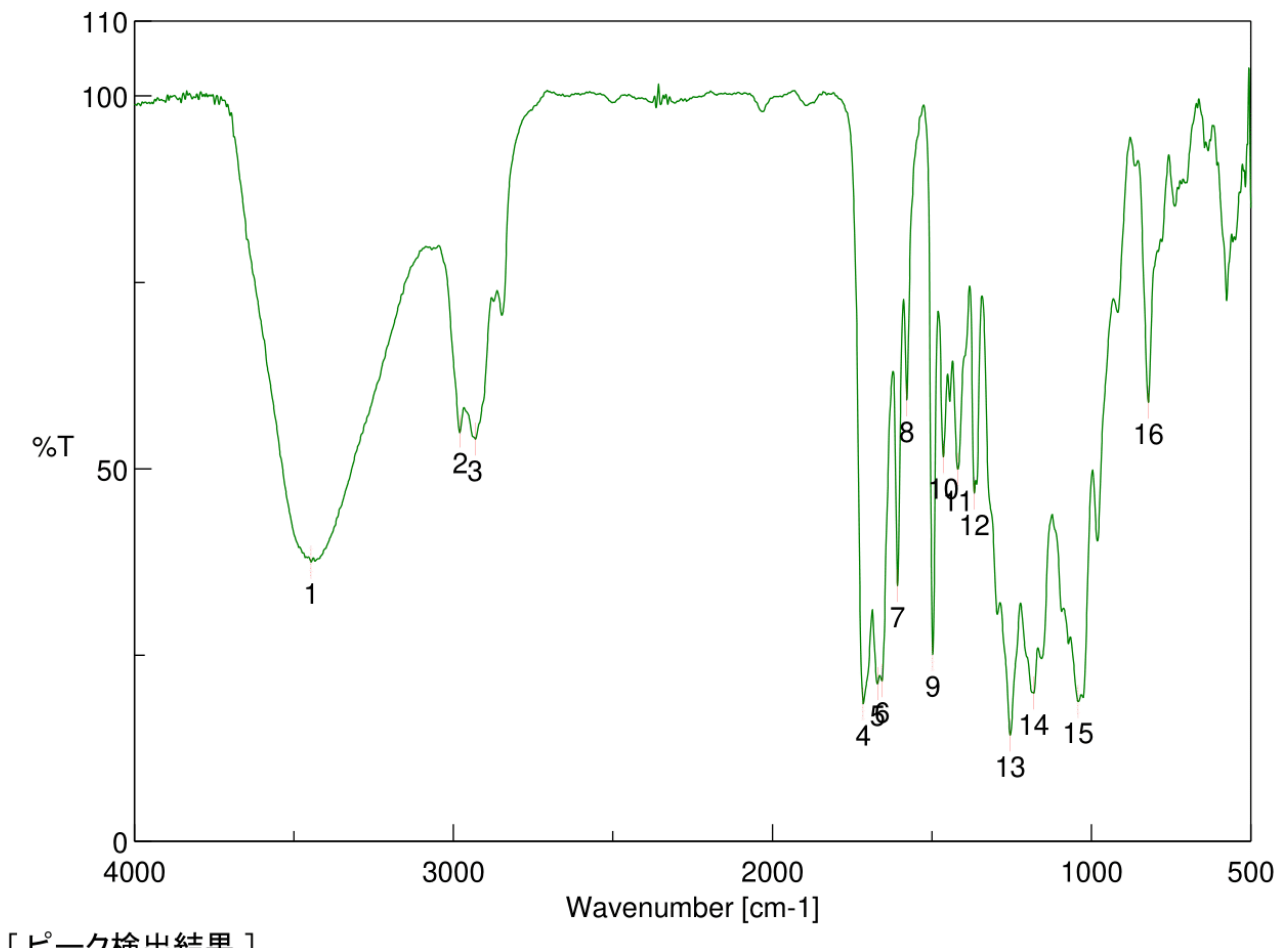

\begin{tabular}{llllll}
\multicolumn{2}{l}{ [ピーク検出結果 ] } & \multicolumn{3}{l}{. } \\
No. & 位置 & 強度 & No. & 位置 & 強度 \\
1 & 3446.17 & 37.4674 & 2 & 2978.52 & 54.981 \\
3 & 2931.27 & 53.9445 & 4 & 1715.37 & 18.4667 \\
5 & 1670.05 & 21.1651 & 6 & 1655.59 & 21.527 \\
7 & 1607.38 & 34.2891 & 8 & 1578.45 & 59.1785 \\
9 & 1497.45 & 25.0595 & 10 & 1464.67 & 51.5644 \\
11 & 1418.39 & 49.8881 & 12 & 1367.28 & 46.7084 \\
13 & 1254.47 & 14.2558 & 14 & 1180.22 & 19.8589 \\
15 & 1041.37 & 18.7568 & 16 & 821.527 & 58.8788
\end{tabular}


Z9.'।

0 L 1

$219^{\circ} \mathrm{z}$
$t+99^{\circ}$

$199^{\circ} \mathrm{Z}$

$\left.089^{\circ} \mathrm{7}\right]$

6142

E6L

$518 \%$

Eะ8 $乙$

1982

$0<8.7$

ozt $\varepsilon$

$186^{\circ} \varepsilon$

GEl'b

$0<1$ -

0.1 5

$\left.0 S L^{\prime} G\right]$

$600^{\circ} 9$

$270^{\circ}$

E七0.9

$699^{\circ} 9$

$\left.\angle 89^{\circ} 9\right]$

$90 L^{\circ} 9$

9219 丁

196.

$1969^{\circ}$
$\varepsilon \angle 6^{\circ}$

$\varepsilon \mid \varepsilon^{\circ}-$

$81 \varepsilon$

๑ย' $t$

$0 t \varepsilon^{\circ} L$

$\varepsilon S E: L$

$09 \varepsilon 2$

$L t \cdot L$

$80^{\circ} L-$

$G L L-$

$6 G L \cdot L$

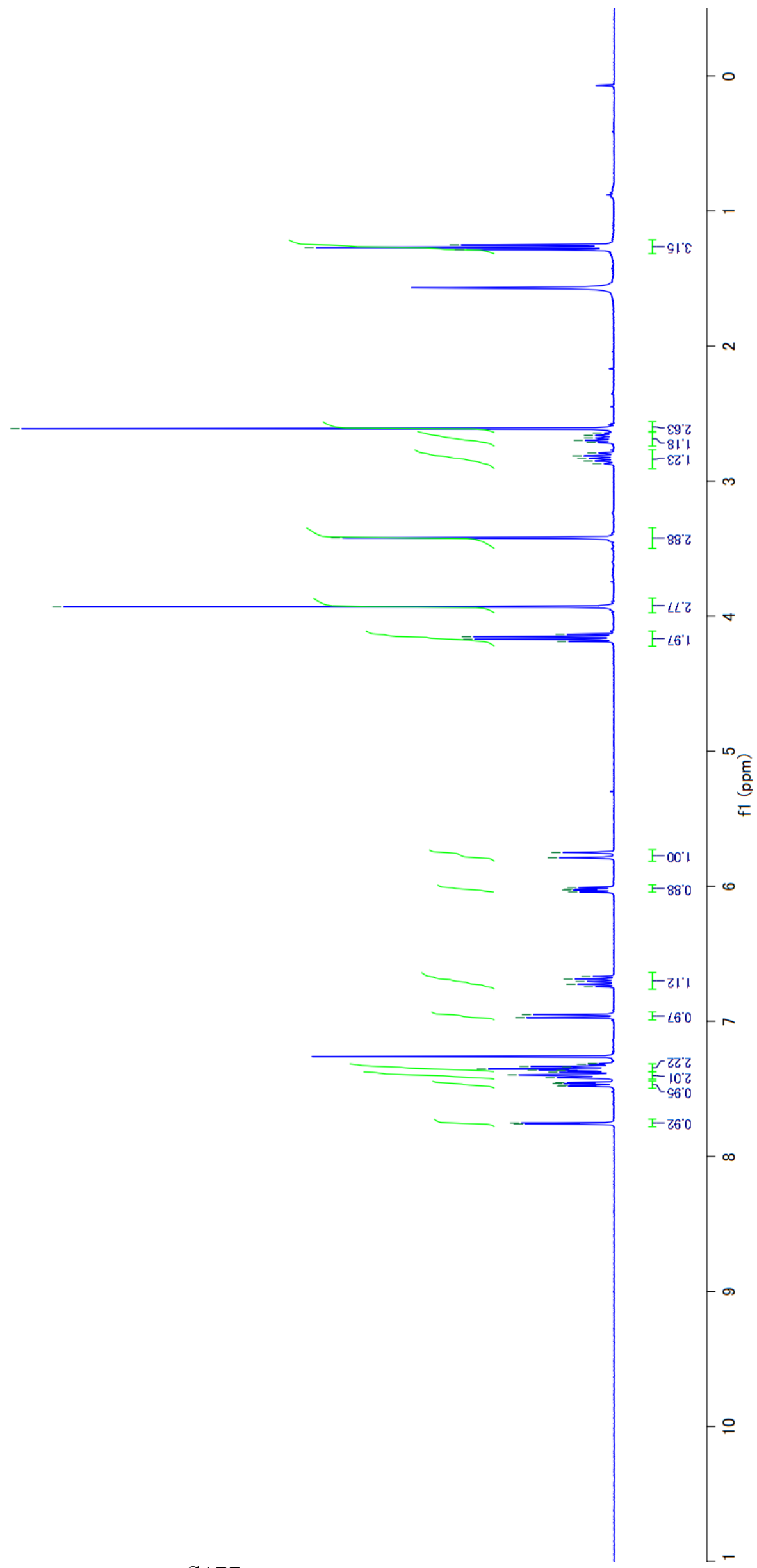




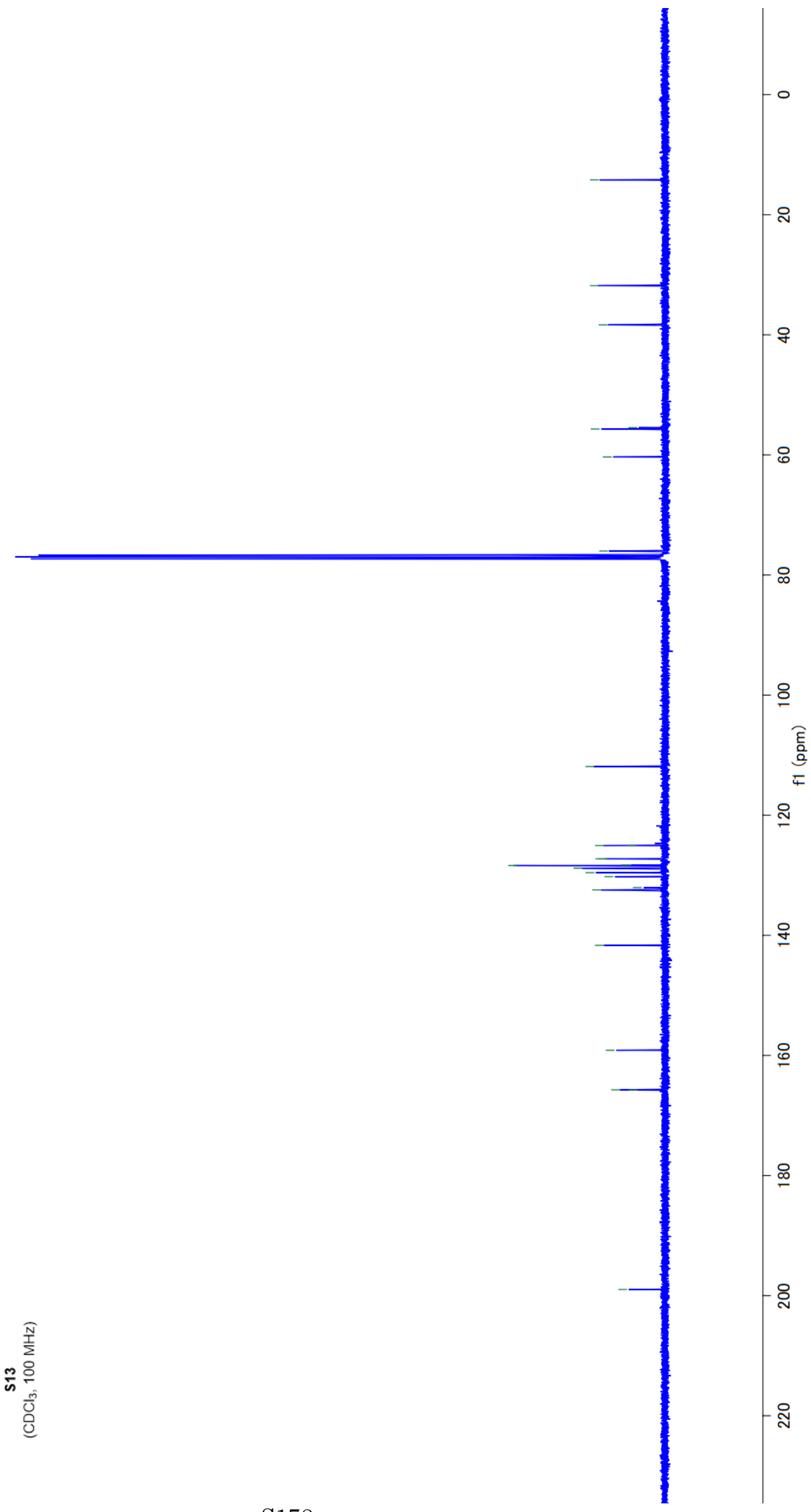




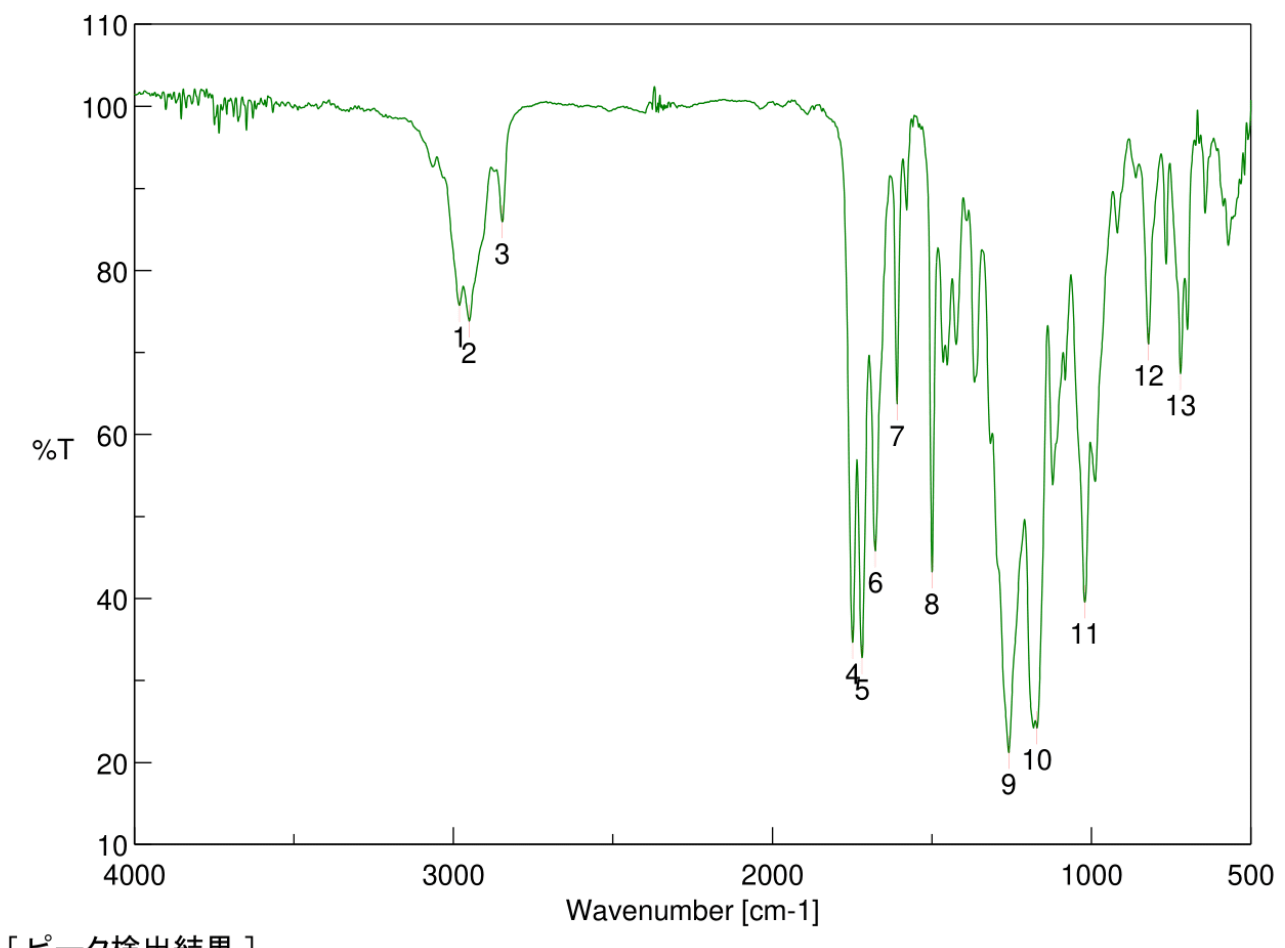

[ピーク検出結果 ]

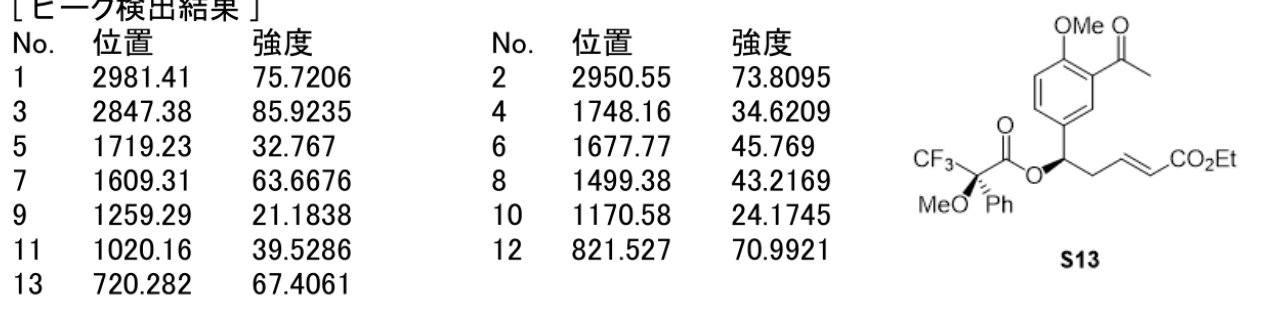


109

$616^{\circ} \varepsilon-$

zsi't

0 L ${ }^{\circ} \mathrm{-}$

$881^{\circ} \mathrm{f}$

$\left.\begin{array}{l}098^{\circ} \mathrm{G} \\ 668^{\circ} \mathrm{s} \\ 906^{\circ} \mathrm{G}\end{array}\right]$

$9+66^{\circ} \mathrm{G}$

896.9

$086^{\circ} 9$

$26 L^{\circ} 9$

$018^{\circ} 9$

$6 \mathrm{ZB}^{\circ}$ ]

$678^{\circ}$
$\angle 98^{\circ}$

$968^{\circ} 9$

$816^{\circ} 9$

$+82: L$

$\varepsilon 0 \varepsilon^{\circ} i$

I $2 \varepsilon^{\circ} L$

0ะร' $L$

Eะะ' $L$

tbE' $L$

OSE 2

$99 \varepsilon^{\circ} L-$

$1 L \varepsilon^{\prime} L-$

$88 \varepsilon^{\circ} L$

$219 \%$

$819^{\circ} \mathrm{L}$

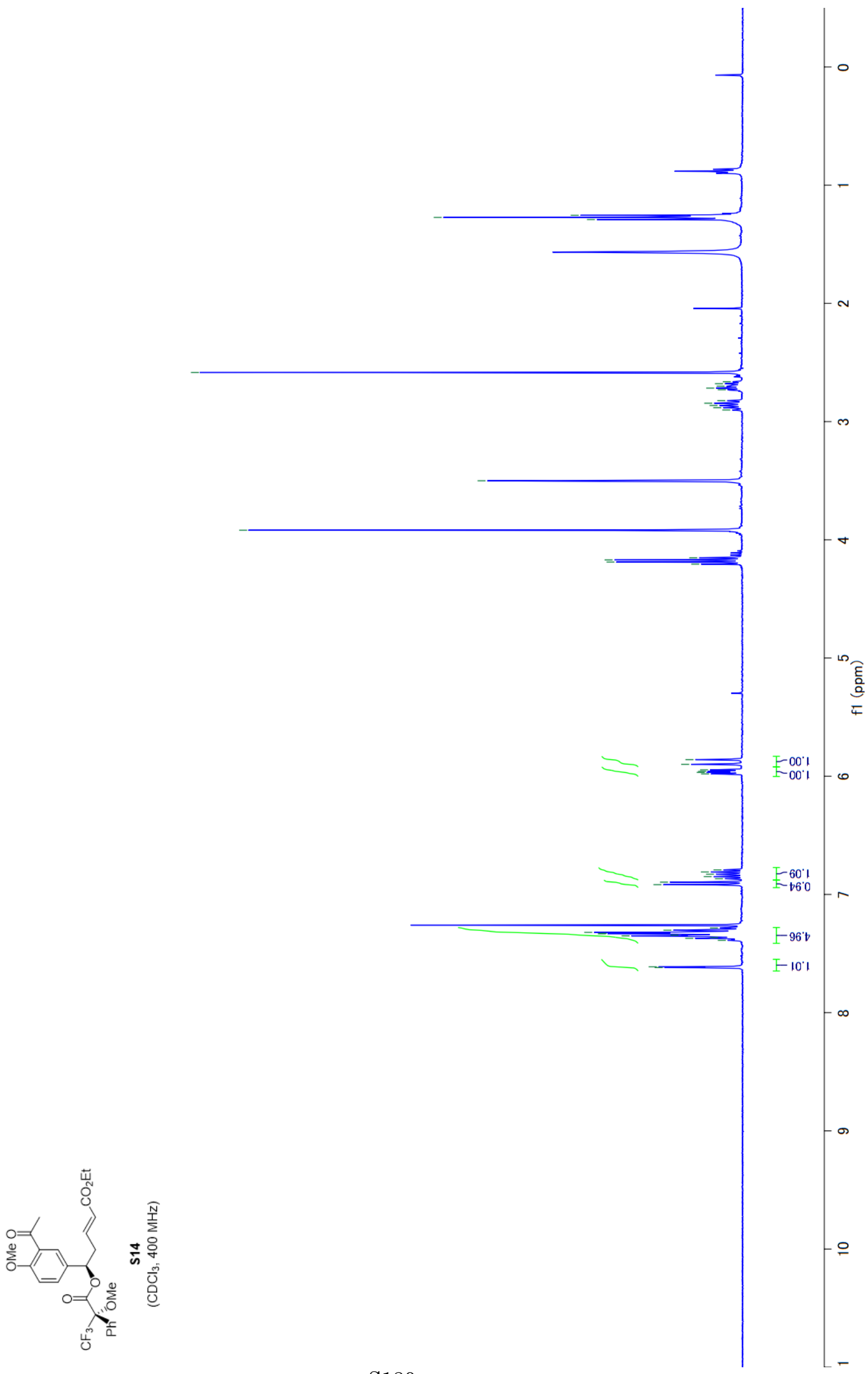


$02+51$

$\downarrow t \cdot \varepsilon-$

ว૬'8६-

99. 99 丁

$69^{\circ} 99$

$t 5.9 L-$
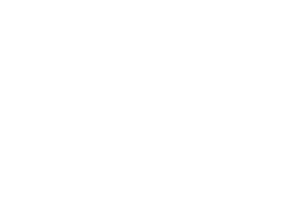

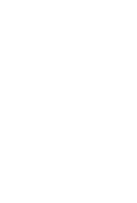

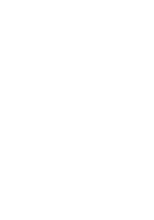

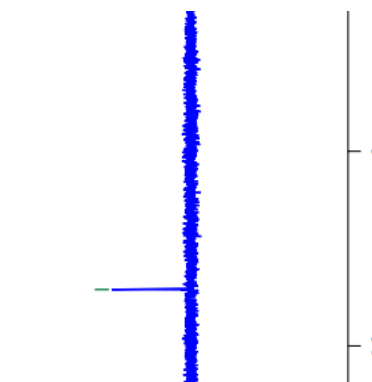




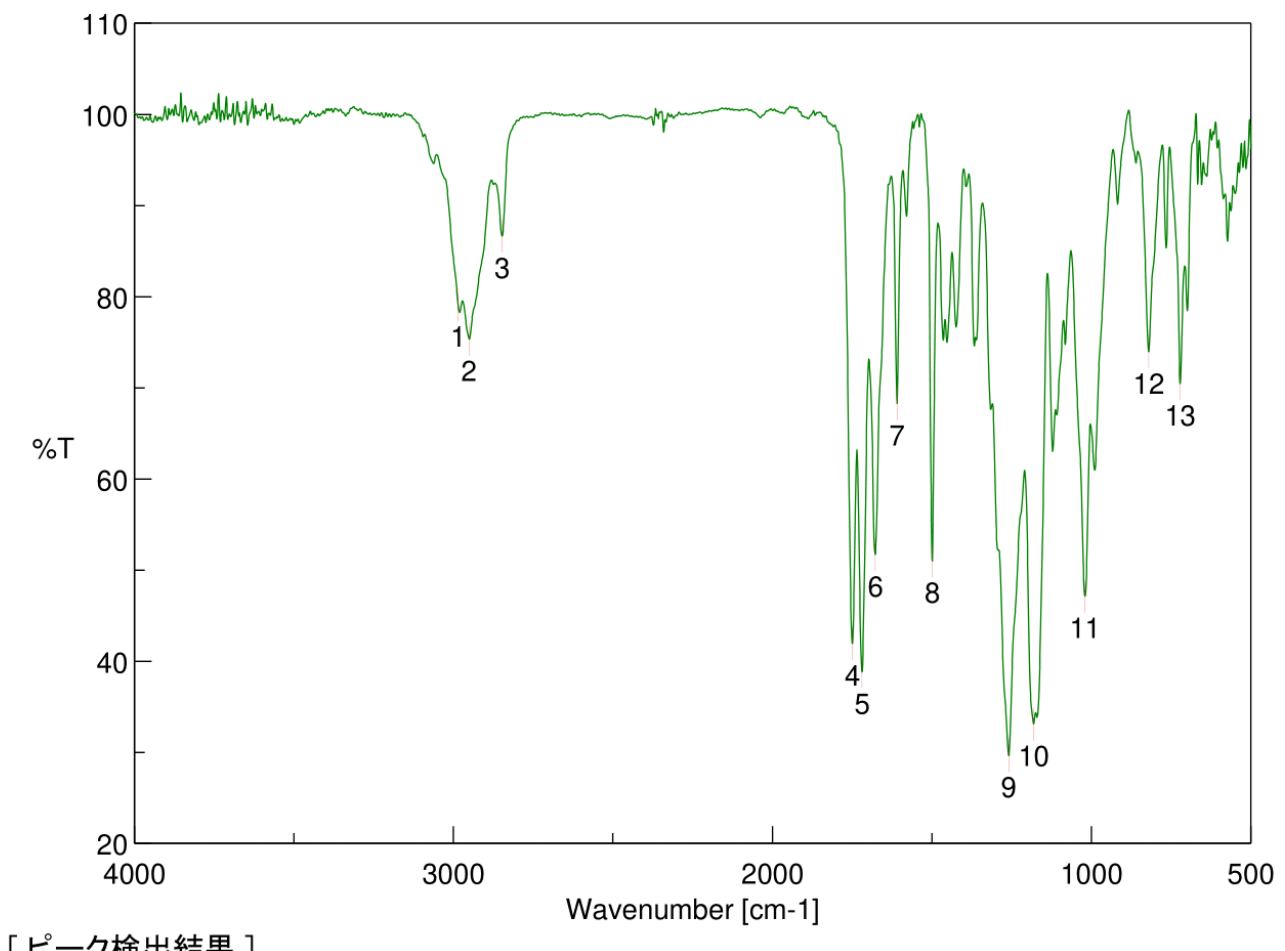

[ピーク検出結果 ]

$\begin{array}{lllllll}\text { No. } & \text { 位置 } & \text { 強度 } & \text { No. } & \text { 位置 } & \text { 強度 } & \\ 1 & 2985.27 & 79.051 & 2 & 2950.55 & 75.3096 & \\ 3 & 2847.38 & 86.6201 & 4 & 1749.12 & 41.9249 \\ 5 & 1719.23 & 38.8099 & 6 & 1677.77 & 51.6861 & \\ 7 & 1609.31 & 68.2234 & 8 & 1498.42 & 50.9727 \\ 9 & 1259.29 & 29.6186 & 10 & 1181.19 & 33.0828 & \\ 11 & 1020.16 & 47.1059 & 12 & 820.563 & 73.9375 & \\ 13 & 721.247 & 70.4506 & & & & \end{array}$




\section{Reference}

(1) Alexander, M. The Transition Metal-Catalyzed Michael Reaction: Enantioselectivity and Vinylogous Donors. B.S. Thesis, The Technical University Berlin, 2001.

(2) (a) Zhang, X. M.; Bordwell, F. G. Equilibrium Acidities and Homolytic Bond Dissociation Energies of the Acidic C-H Bonds in P-Substituted Triphenylphosphonium Cations. J. Am. Chem. Soc. 1994, 116, 968-972. (b) Mao, C.; Wang, Z.; Wang, Z.; Ji, P.; Cheng, J. P. Weakly Polar Aprotic Ionic Liquids Acting as Strong Dissociating Solvent: A Typical "Ionic Liquid Effect" Revealed by Accurate Measurement of Absolute $\mathrm{p} K_{\mathrm{a}}$ of Ylide Precursor Salts. J. Am. Chem. Soc. 2016, 138, 5523-5526.

(3) Fu, Y.; Wang, H. J.; Chong, S. S.; Guo, Q. X.; Liu, L. An Extensive Ylide Thermodynamic Stability Scale Predicted by First-Principle Calculations. J. Org. Chem. 2009, 74, 810-819.

(4) Bordwell, F. G. Equilibrium Acidities in Dimethyl Sulfoxide Solution. Acc. Chem. Res. 1988, 21, 456-463.

(5) Ng, S. M.; Bader, S. J.; Snapper, M. L. Solvent-Controlled Intramolecular [2+2] Photocycloadditions of $\alpha$-Substituted Enones. J. Am. Chem. Soc. 2006, 128, 7315-7319.

(6) Zhang, J. T.; Qi, X. L.; Chen, J.; Li, B. S.; Zhou, Y. B.; Cao, X. P. Total Synthesis of Malyngamides K, L, and 5"-epi-C and Absolute Configuration of Malyngamide L. J. Org. Chem. 2011, 76, 3946-3959.

(7) Zhan, G.; He, Q.; Yuan, X.; Chen, Y. C. Asymmetric Direct Vinylogous Michael Additions of Allyl Alkyl Ketones to Maleimides through Dienamine Catalysis. Org. Lett. 2014, 16, 6000-6003.

(8) Liotta, D.; Saindane, M.; Brnum, C.; Ensley, H.; Balakrishnan. Reactions Involving Selenium Metal as an Electrophile. 2. A General Procedure for the Preparation of Unsaturated $\beta$-Dicarbonyl Compounds. Tetrahedron Lett. 1981, 22, 3043-3046. 